\title{
Honeywell
}

\section{Design of Experiments to Determine Causes of Flex Cable Solder Wicking, Discoloration and Hole Location Defects}

Federal Manufacturing \& Technologies

Larry Wolfe

\section{KCP-613-8569}

Published March 2009

Final Report

Approved for public release; distribution is unlimited.

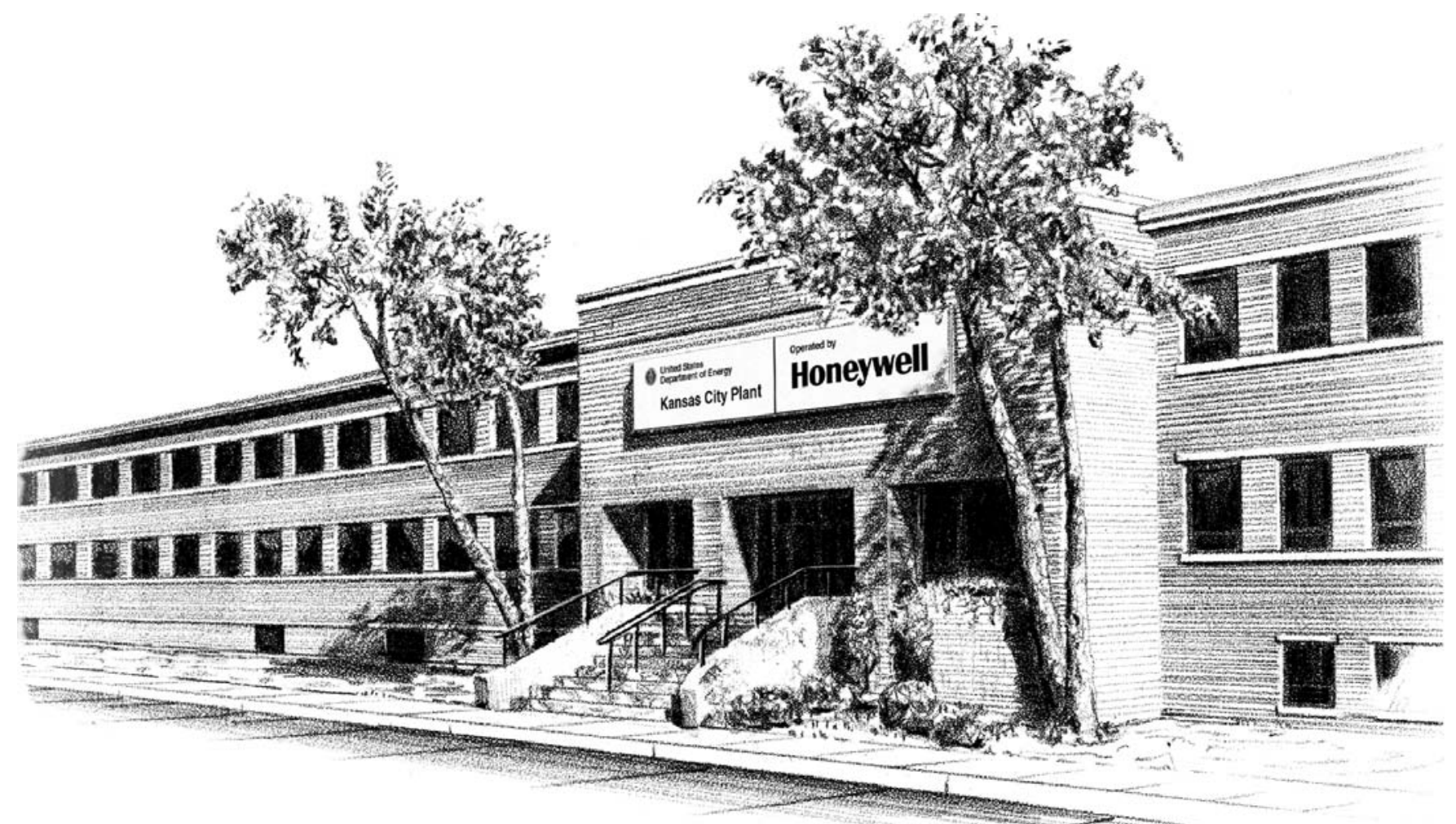

Prepared under prime contract DE-ACO4-01AL66850 for the United States Department of Energy 


\section{DISCLAIMER}

This report was prepared as an account of work sponsored by an agency of the United States Government. Neither the United States Government nor any agency thereof, nor any of their employees, makes any warranty, express or implied, or assumes any legal liability or responsibility for the accuracy, completeness, or usefulness of any information, apparatus, product, or process disclosed, or represents that its use would not infringe privately owned rights. Reference herein to any specific commercial product, process or service by trade names, trademark, manufacturer, or otherwise, does not necessarily constitute or imply its endorsement, recommendation or favoring by the United States Government or any agency thereof. The views and opinions of authors expressed herein do not necessarily state or reflect those of the United States Government or any agency thereof.

All data prepared, analyzed and presented has been developed in a specific context of work and was prepared for internal evaluation and use pursuant to that work authorized under the reference contract. Reference herein to any specific commercial product, process or service by trade name, trademark, manufacturer, or otherwise, does not necessarily constitute or imply its endorsement, recommendation or favoring by the United States Government, any agency thereof or Honeywell Federal Manufacturing \& Technologies, LLC.

Printed in the United States of America.

This report has been reproduced from the best available copy.

Available to DOE and DOE contractors from the Office of Scientific and Technical Information, P.O. Box 62, Oak Ridge, Tennessee 37831; prices available from (865) 576-8401, FTS 626-8401.

Available to the public from the National Technical Information Service, U.S. Department of Commerce, 5285 Port Royal, Rd., Springfield, Virginia 22161, (703) 487-4650.

A prime contractor with the United States Department of Energy under Contract Number DE-AC04-O1AL66850

\author{
Honeywell Federal Manufacturing \& Technologies \\ P.O. Box 419159 \\ Kansas City, Missouri, 64141-6159
}




\title{
Honeywell
}

KCP-613-8569

Distribution Category UC-XX

Approved for public release; distribution is unlimited.

Design of Experiments to Determine Causes of Flex Cable Solder Wicking, Discoloration and Hole Location Defects

\author{
Larry Wolfe \\ Published March 2009 \\ Final Report \\ Larry Wolfe, Project Leader \\ Project Team: \\ Mike McHenry \\ Dave Stockdale \\ Tonya Burke
}





\section{Contents}

Section Page

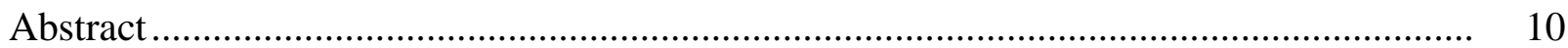

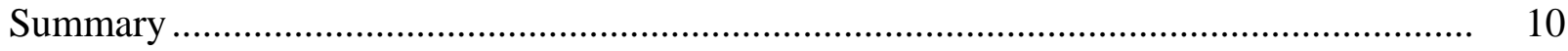

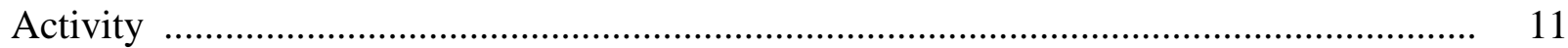

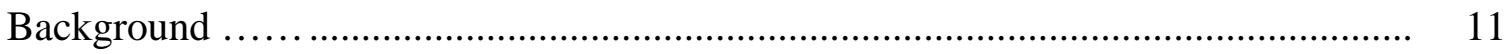

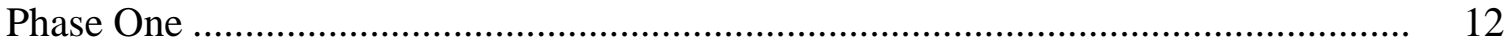

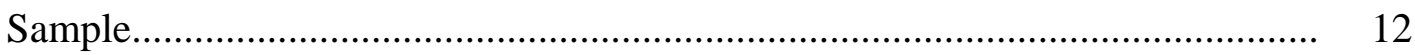

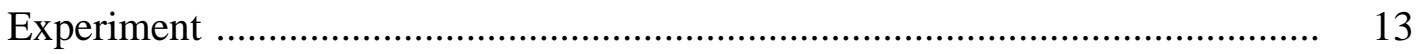

Analysis of Discoloration/Solder Wicking Information .............................. 13

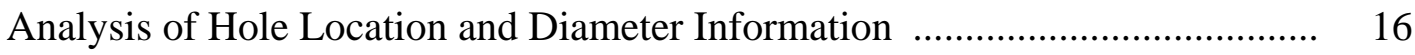

Panel Movement in the X Axis .................................................................. 18

Panel Movement in the Y Axis ................................................................ 20

Validation of Phase One Data and Analysis ................................................ 22

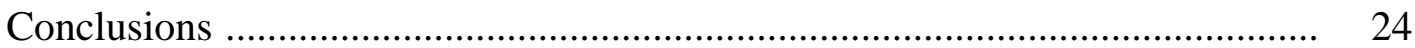

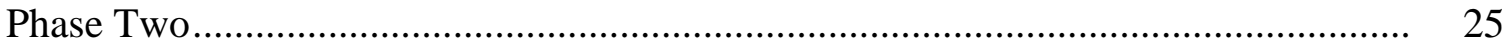

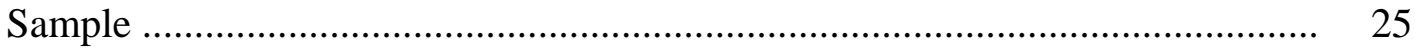

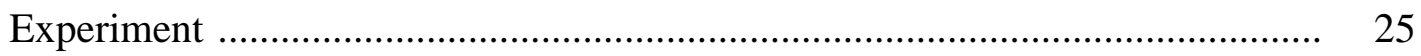

Hole Location Analysis .......................................................................... 26

Adhesion Quality Analysis ............................................................... 27

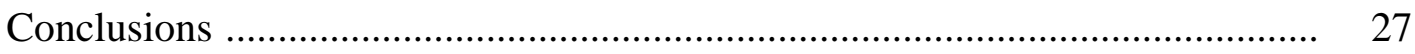

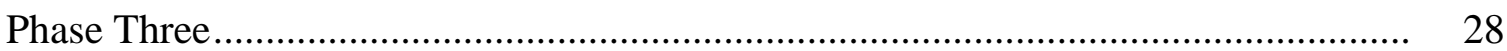

Sample (Two Ounce Copper Laminate) …................................................ 28 


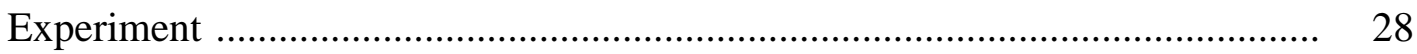

Hole Location Analysis .................................................................... 29

Adhesion Quality Analysis ................................................................... 31

Statistical Analysis of Soft Press Data ...................................................... 36

Conclusions ….................................................................................. 44

Sample (One Ounce Copper Laminate)....................................................... 44

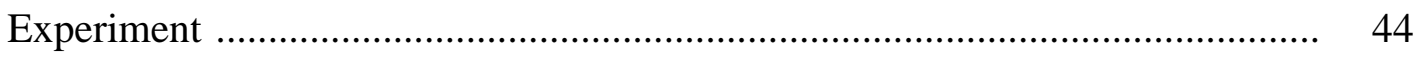

Adhesion Quality Analysis ................................................................. 45

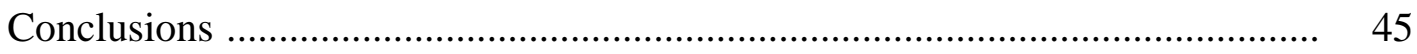

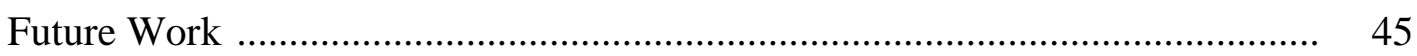

Appendices

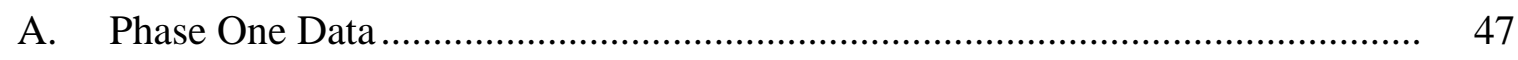

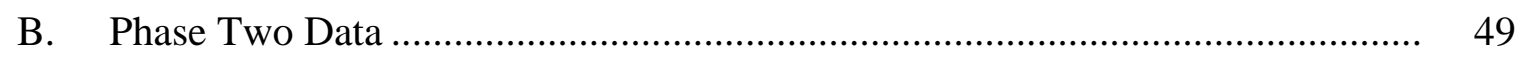

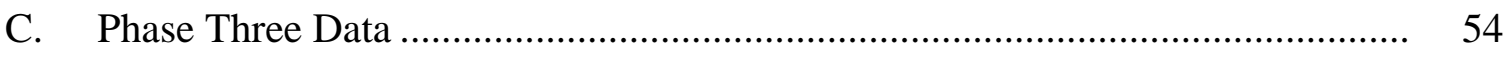

D. Manufacturing Work Instructions........................................................... 68

E. Trace Laboratories Test Reports ............................................................. 85 


\section{Illustrations}

Figure Page

1 Hard Press Pad Stackup ................................................................................. 12

2 Soft Press Pad Stackup............................................................................... 12

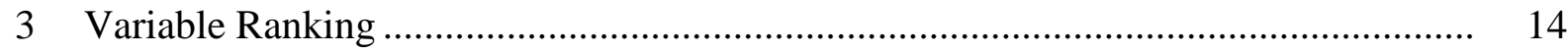

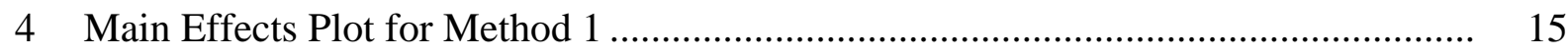

$5 \quad$ Interaction Plot for Method 1......................................................................... 16

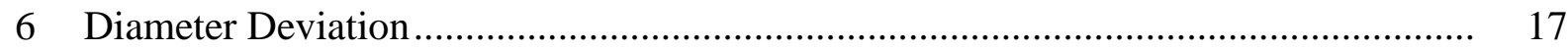

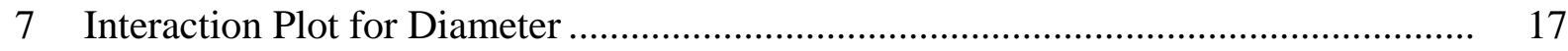

8 Main Effects Plot for Diameter..................................................................... 18

9 Between/Within Capability of Diameter ........................................................ 18

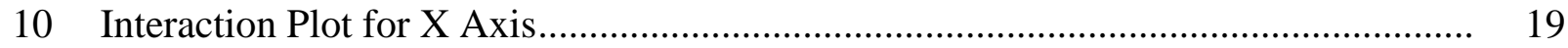

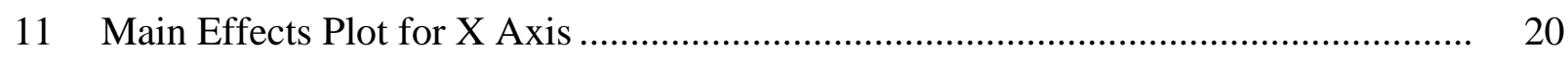

12 Interaction Plot for Y Axis...................................................................... 21

13 Main Effects Plot for Y Axis ..................................................................... 21

14 Marginal Plot of Y Axis vs. X Axis................................................................. 22

15 Variable Ranking of Validation Data ................................................................... 23

16 Original, Validation and Combined Variable Ranking......................................... 24

17 Parts Per Million Stretch of Sample Panels ........................................................ 26

18 Panel Ranking ......................................................................................... 27

19 Lamination Evaluation Panel Stretch

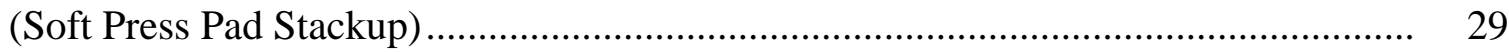

20 Lamination Evaluation Panel Stretch (Hard Press Pad Stackup/Soft Press Pad Stackup) ................................................ 30 
21 Annular Rings .............................................................................................. 32

22 Annular Ring.................................................................................................... 33

23 Annular Ring with Dark Halo .............................................................................. 34

24 Annular Rings with Dark Halos.............................................................................. 35

25 Main Effects Plot for X Axis .............................................................................. 37

26 Interaction Plot for X Axis............................................................................ 37

27 Main Effects Plot for Y Axis ........................................................................... 38

28 Interaction Plot for Y Axis............................................................................... 39

29 Main Effects for Solder Wicking .............................................................................. 40

$30 \quad$ Interaction Plot for Solder Wicking ........................................................................ 40

31 Main Effects Plot for Dark Halos ........................................................................ 41

32 Interaction Plot for Dark Halos......................................................................... 42

33 Boxplot of PPM Y by Pressure.......................................................................... 53

34 Boxplot of Discoloration by Pressure ...................................................................... 53

\section{Tables}

Number

Page

1 Panel Ranking ................................................................................................ 31

2 Phase Three DoE Results.......................................................................................

3 Panel Ranking .............................................................................................. 46

4 Hole Location................................................................................................. 4

$5 \quad$ Hole Analysis ................................................................................................... 47

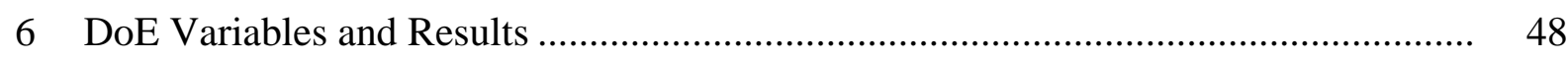


$7 \quad$ Post Laminate Hole Location X Values......................................................................... 49

8 Post Laminate Hole Location Y Values........................................................................... 50

9 Adhesion Quality Data....................................................................................... 51

10 Panels 1-16 Rankings X Values........................................................................

11 Panels 17-32 Rankings X Values........................................................................... 55

12 Panels 1-16 Rankings Y Values.......................................................................... 56

13 Panels 17-32 Rankings Y Values.............................................................................. 57

14 Panels 1-8 Delamination/Solder Wicking Results ........................................................ 58

15 Panels 9-16 Delamination/Solder Wicking Results ........................................................ 59

16 Panels 17-24 Delamination/Solder Wicking Results.................................................... 60

17 Panels 25-32 Delamination/Solder Wicking Results................................................ 61

18 Panel Rankings.................................................................................................. 62

19 Panels 1-9 Delamination/Solder Wicking Results

(One Ounce Copper) .................................................................................................... 63

20 Panels 10-18 Delamination/Solder Wicking Results

(One Ounce Copper) ................................................................................................... 64

21 Panels 19-27 Delamination/Solder Wicking Results

(One Ounce Copper) .................................................................................................. 65

22 Panels 28-36 Delamination/Solder Wicking Results

(One Ounce Copper) .................................................................................................. 66

23 Panel Rankings.................................................................................................. 67 


\begin{abstract}
Design of Experiments (DoE) were developed and performed in an effort to discover and resolve the causes of three different manufacturing issues; large panel voids after Hot Air Solder Leveling (HASL), cable hole locations out of tolerance after lamination and delamination/solder wicking around flat flex cable circuit lands after HASL. Results from a first DoE indicated large panel voids could be eliminated by removing the pre-HASL cleaning. It also revealed eliminating the pre-HASL bake would not be detrimental when using a hard press pad lamination stackup. A second DoE indicated a reduction in hard press pad stackup lamination pressure reduced panel stretch in the y axis approximately 70\%. A third DoE illustrated increasing the pre-HASL bake temperature could reduce delamination/solder wicking when using a soft press pad lamination stackup.
\end{abstract}

\title{
Summary
}

Flat flex cable lamination entails the use of a press pad stackup placed between lamination press platens. There are two lamination press pad stackups presently being utilized in production, hard press pad and soft press pad. The hard press pad stackup has been used almost exclusively for the past twenty years. A soft press pad stackup has recently been utilized to reduce panel stretching and adhesive squeeze out. The differing ways these two press pad stackups affect the quality of lamination adhesion is the basis for the activities described in this report. These activities were conducted in an effort to resolve manufacturing issues related to the quality of adherence after lamination.

In July of 2006 after completion of a department's Hot Air Solder Leveling (HASL) process, large voids and/or delamination appeared immediately on several lots of panels, Main and Aux cables. A full factorial DoE was conducted in an effort to understand the cause of the voids/delamination. Utilizing the most likely variables, lamination stackup, bake, and clean did not recreate the large voids, but did illustrate other possible processing modifications of interest.

The hard press pad stackup obtained the best results with the least amount of delamination/solder wicking when panels were not baked or cleaned prior to HASL. This was also the combination of variable factors exhibiting the best adhesion of all variables for both press pad stackups. The soft press pad stackup obtained the best results when panels were baked and either cleaned or not cleaned prior to HASL. All twenty-four cables laminated using the soft press pad stackup that were not baked had significant amounts of solder wicking under the coverlay. This implies inadequate adhesion.

A second manufacturing issue related to out of tolerance hole locations became apparent on the Firing Set flex cables, the first to require hole location measurement after plating and lamination. A full factorial DoE was performed to resolve this problem by reducing pressure during lamination. Based on the results, a reduction in lamination pressure from 190 tons to 90 tons should not affect the quality of adhesion between coverlay and copper laminate, but could reduce panel stretch in the y axis up to $70 \%$. 
The final issue addressed by this report was the appearance of minor delamination/solder wicking around circuit lands after HASL when using the soft press pad stackup. In an effort to determine which variable or variables had the greatest effect on delamination/solder wicking, a fractional factorial DoE was developed. The experiment varied lamination pressure, lamination temperature, lamination time, pre-HASL bake time and temperature. Two ounce copper laminate cables used for this experiment exhibited no coverlay delamination or solder wicking around circuit lands when an increased bake temperature was used for the HASL pre-bake. A shorten bake time at the increased bake temperature also did not exhibit any coverlay delamination or solder wicking.

Performing the second part of this experiment with one ounce copper laminate cables, there was an increase in delamination/solder wicking with all variable combinations. Data analysis indicated longer HASL pre-bake times will produce less delamination/solder wicking. This data implies different processing for two and one ounce copper laminates.

\section{Activity}

The activities contained in this report were conducted in an effort to resolve manufacturing issues related to the quality of adherence after lamination. These activities are grouped into phases which are described as:

Phase One - A full factorial DoE conducted in an effort to understand why several lots of part numbers, Main and Aux cables, exhibited voids/delamination after HASL.

Phase Two - A full factorial DoE performed to resolve flex cable hole location out of tolerance conditions. This issue became apparent on the Firing Set flex cables, the first to require hole location measurement after plating and lamination.

Phase Three - A fractional factorial DoE performed to determine if variations in lamination and pre-HASL process variables affect coverlay delamination/solder wicking around circuit lands when using the lamination soft press pad stackup.

\section{Background}

Flat flex cable lamination entails the use of a press pad stackup placed between lamination press platens. To adhere coverlay to copper laminate the cable panels are placed in the center of a press pad stackup and then placed between the press platens. There are two lamination press pad stackups presently being utilized in production, hard press pad and soft press pad. The hard press pad stackup has been used almost exclusively for the past twenty years. A soft press pad stackup has been utilized recently on cables to reduce panel stretching and adhesive squeeze out. The different press pad stackups and their contents are listed below. 
Hard press pad stackup

Metal plate

Rubber

Teflon

Parts

Teflon

Rubber

Metal plate

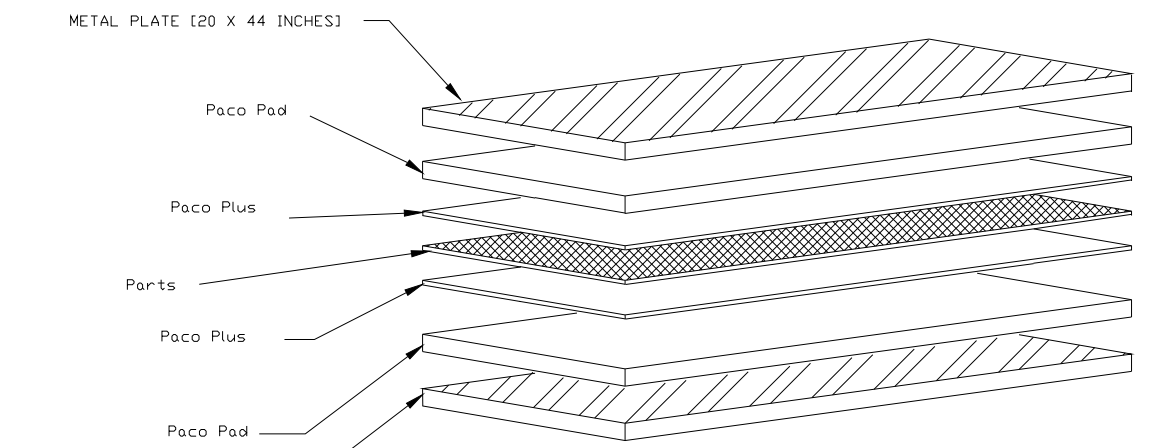

Soft press pad stackup

Metal plate

Pacopad

Paco Plus

Parts

Paco Plus

Pacopad

Metal plate

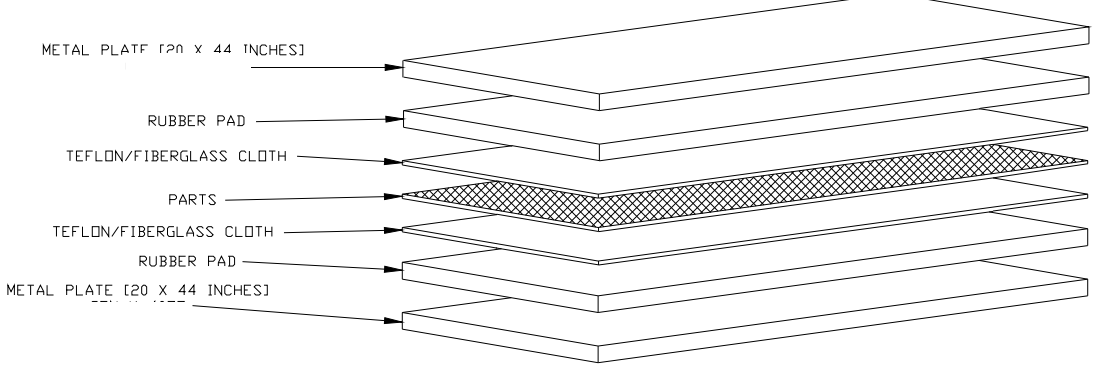

Figure 2. Soft Press Pad Stackup

All activities and experiments contained in this report utilized one or both of the above press pad stackups.

\section{Phase One}

In July of 2006 after completion of a department's HASL process, large voids and/or delamination appeared immediately on several lots of panels, Main and Aux cables. This DoE's objective was to determine the cause by recreating the large voids in a structured experiment.

Hole diameter and location Coordinate Measuring Machine (CMM) data also was collected from the sample panels used for this experiment. This data provided insight into the amount of panel stretch resulting from the use of a hard press pad stackup during lamination.

\section{Sample}

A twelve-by-twelve inch, two ounce copper, double-sided laminate sample was generated, which contained two circuit patterns. The coverlay laminated to both sides of the laminate samples was two mil polyimide/two mil pyralux adhesive. 


\section{Experiment}

A full factorial DoE was developed to determine if variations in lamination press pad stackup, bake or cleaning resulted in large voids/delamination after HASL. Since the objective was to recreate large voids if possible extreme values of each variable was used.

The diagram below illustrates the process flow from lamination through HASL and the DoE variables/factors. Four panels/eight parts were processed for each variable factor combination resulting in a total of thirty-two panels/sixty-four parts.

\begin{tabular}{|c|c|c|c|}
\hline $\begin{array}{l}\text { Lamination } \\
\text { TMP Press }\end{array}$ & Bake & Clean & $\begin{array}{l}\text { Hot Air Solder } \\
\text { Leveling }\end{array}$ \\
\hline Hard press pad stackup & $\begin{array}{l}\text { Bake } \\
\text { No bake }\end{array}$ & $\begin{array}{l}\text { Clean } \\
\text { No clean } \\
\text { Clean } \\
\text { No clean }\end{array}$ & \\
\hline Soft press pad stackup & $\begin{array}{l}\text { Bake } \\
\text { No Bake }\end{array}$ & $\begin{array}{l}\text { Clean } \\
\text { No clean } \\
\text { Clean } \\
\text { No clean }\end{array}$ & \\
\hline
\end{tabular}

After lamination each panel's nine .093 holes were measured for diameter and location by a department's CMM. The results were recorded and are displayed in Appendix A.

\section{Analysis of Discoloration/Solder Wicking Information}

The HASL process is considered a test of adhesion quality between the coverlay and laminate panel. Therefore, large areas of delamination could be a lack of adhesion/contamination or insufficient adhesion. These areas of delamination have in the past been identified as discoloration, delamination or solder wicking. To score this experiment's panels objectively and consistently these three characteristics were considered equal. The magnitude of these characteristics was used to determine a numerical score for each part.

Each cable on each panel was inspected and the magnitude of discoloration/solder wicking was measured. If there was more then one area of discoloration/solder wicking, the magnitude of each area was added together to provide a total cable discoloration/solder wicking value. Then each total cable value was assigned a ranking as listed below.

$\begin{array}{clc}\text { Ranking value } & \text { Amount of discoloration/solder wicking } \\ 1 & = & 1 \text { to } 10 \text { mils }(.001 \text { to } .010) \\ 3 & = & 10 \text { to } 20 \text { mils }(.010 \text { to } .020) \\ 7 & = & 20 \text { to } 30 \text { mils }(.020 \text { to } .030) \\ 10 & = & \text { Over } 30 \text { mils }(.030)\end{array}$


Cable rankings were summed to produce an overall variable ranking as shown in the following illustration.

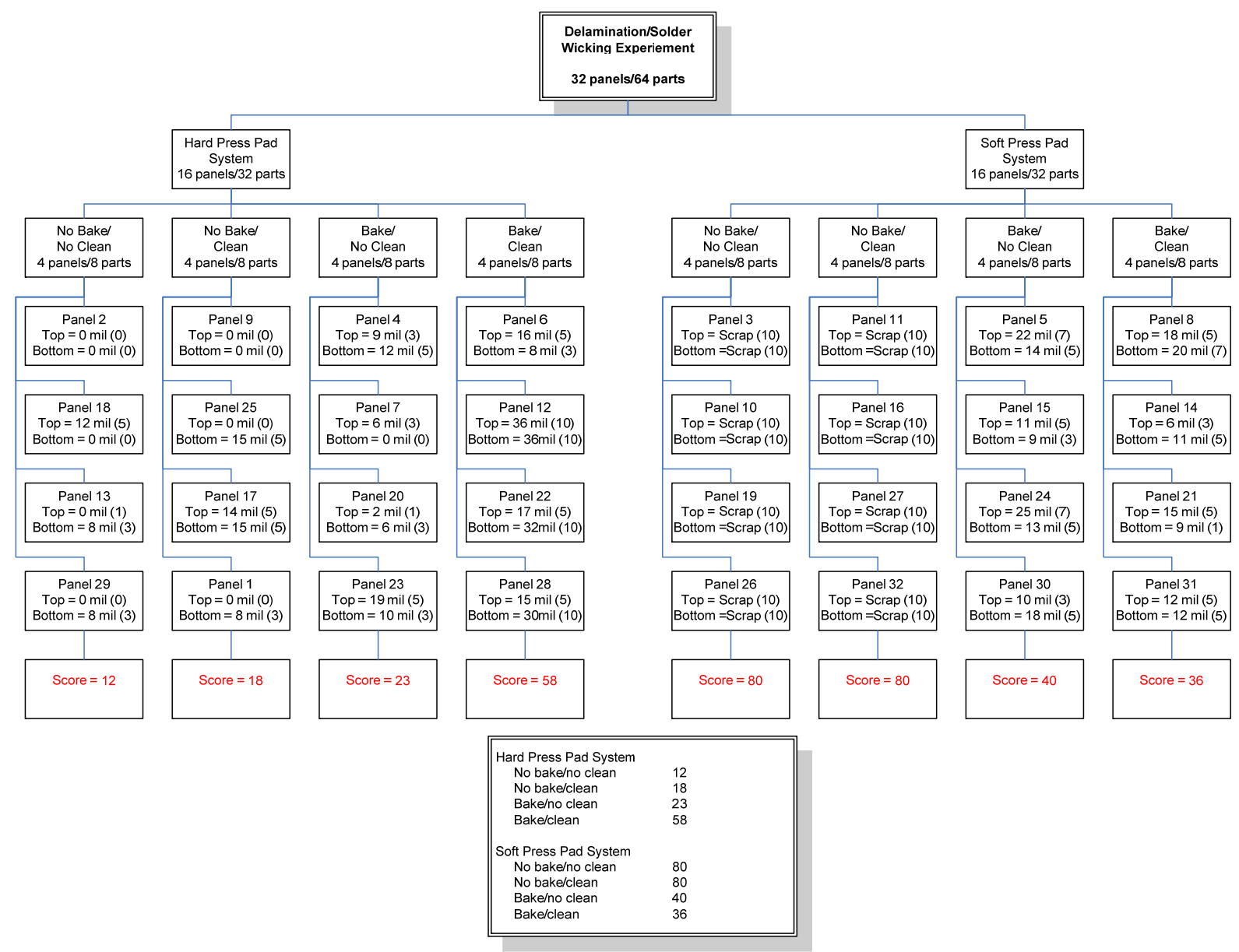

Figure 3. Variable Ranking

The hard press pad stackup sample panels not baked or cleaned before HASL had the least amount of discoloration/solder wicking. The soft press pad stackup sample panels had significant solder wicking on the panels not baked and cleaned/not cleaned. 
The amount of delamination/solder wicking was measured in mils and the statistical analysis is shown below. Any P value less than .1 is statistically significant.

$\begin{array}{lrrrrrr}\text { Source } & \text { DF } & \text { Seq SS } & \text { Adj SS } & \text { Adj MS } & F & \text { P } \\ \text { Blocks } & 1 & 108.8 & 108.8 & 108.8 & 1.13 & 0.300 \\ \text { Stackup } & 1 & 7533.8 & 7533.8 & 7533.8 & 78.25 & 0.000 \\ \text { Bake } & 1 & 1262.5 & 1262.5 & 1262.5 & 13.11 & 0.002 \\ \text { Clean } & 1 & 871.5 & 871.5 & 871.5 & 9.05 & 0.007 \\ \text { Stackup*Bake } & 1 & 9418.8 & 9418.8 & 9418.8 & 97.83 & 0.000 \\ \text { Stackup }{ }^{*} \text { Clean } & 1 & 552.8 & 552.8 & 552.8 & 5.74 & 0.026 \\ \text { Bake*Clean } & 1 & 442.5 & 442.5 & 442.5 & 4.60 & 0.045 \\ \text { Error } & 20 & 1925.6 & 1925.6 & 96.3 & & \\ \text { Total } & 31 & 22801.5 & & & & \\ \text { S }=9.81230 & \text { R-Sq }=91.55 \% & \text { R-Sq(adj) }=86.91 \% & \end{array}$

Although several factors were significant the lamination stackup and pre-HASL bake had the greatest effect. The main effect and interaction plots are shown in Figures 4 and 5.

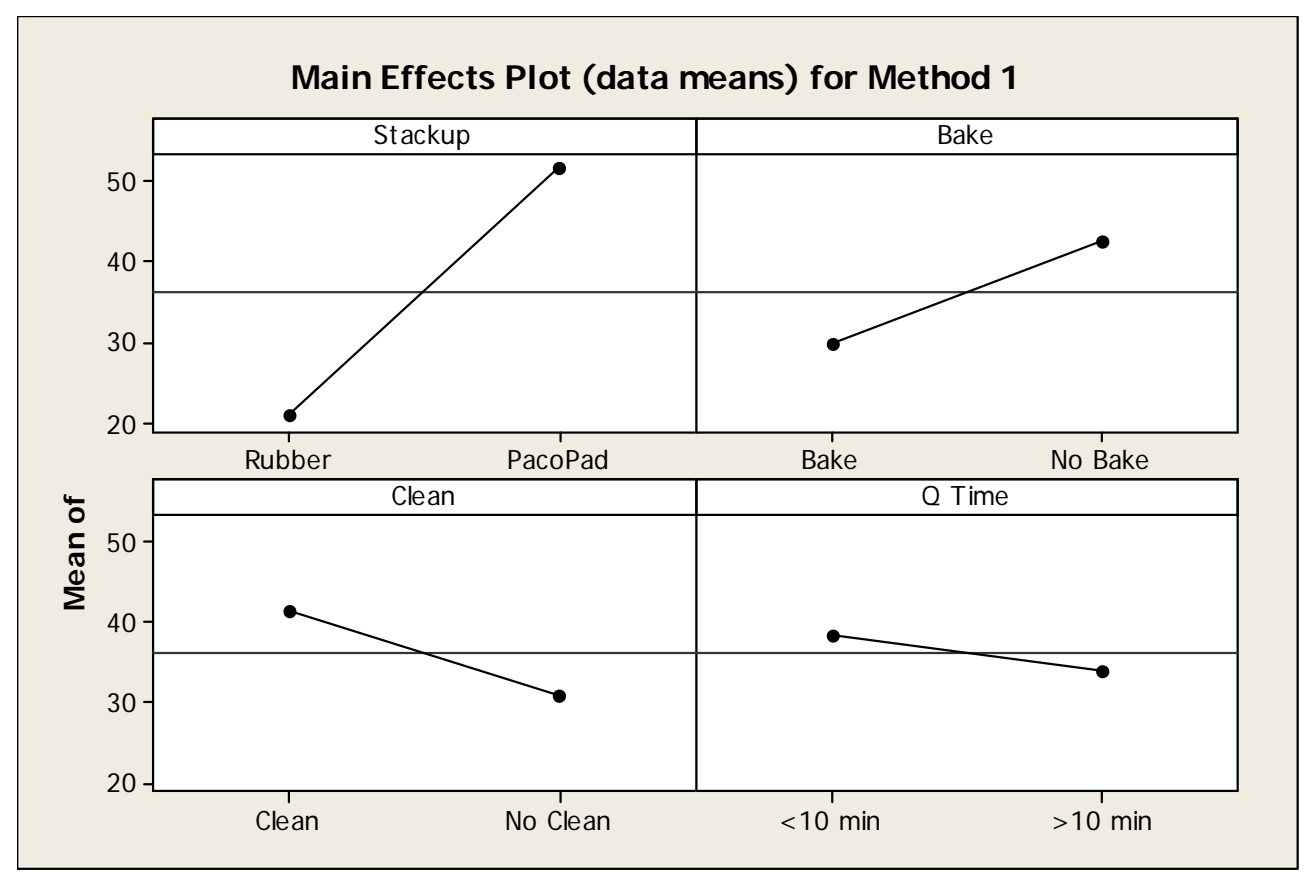

Figure 4. Main Effects Plot for Method 1 


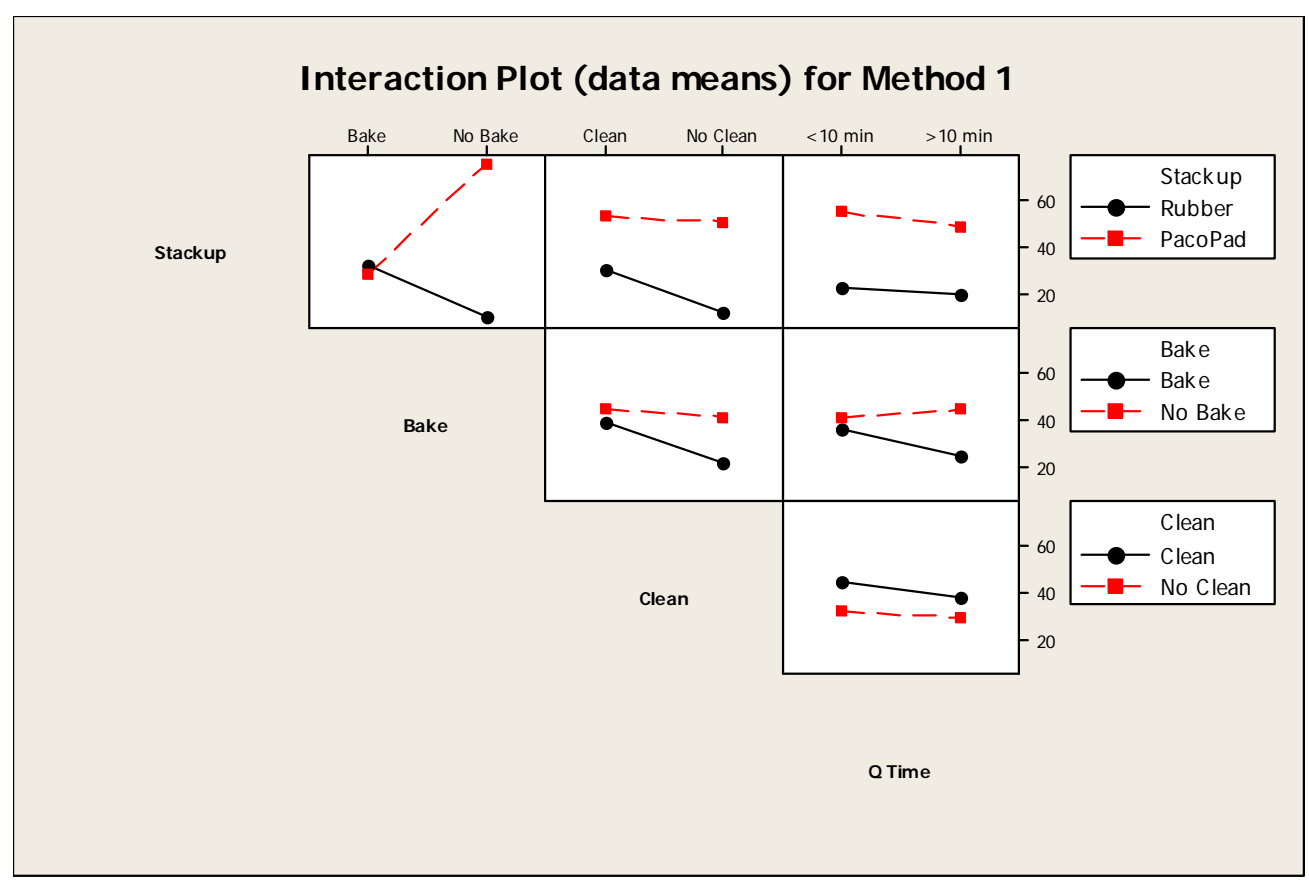

Figure 5. Interaction Plot for Method 1

The main effects plot shows lamination stackup is most significant with pre-HASL bake and clean, as the data indicates at about the same level (13.11 vs 9.05). The only interaction is between the lamination stackup and pre-HASL bake as illustrated in Figure 5.

\section{Analysis of Hole Location and Diameter Information}

The average deviation from drilled hole diameter was -.00057 inches. The statistical data shows none of the parameters had a significant impact on hole diameter. To be significant the P value would have to be less than .1. The data is displayed by panel as a radar chart in Figure 6 and the interaction and main effects plots in Figures 7 and 8. Although the main effects plots show bake and clean affect hole diameter, the amount of deviation is well within what is expected from a non-plated through hole in Kapton as displayed in Figure 9.

\begin{tabular}{|c|c|c|c|c|c|c|}
\hline Source & DF & Seq SS & Adj $S S$ & Adj MS & $\mathrm{F}$ & $\mathrm{P}$ \\
\hline Blocks & 1 & ๑. . ००००००० & $\odot .000 \odot \odot ० \odot$ & ๑. . $0000 \odot ० \odot$ & 0.95 & 0.343 \\
\hline Stackup & 1 & ๑. . $0000 \odot \odot \odot$ & 0.0000000 & ๑. . $00 \odot ० ० ० \odot$ & 0.00 & 0.960 \\
\hline Bake & 1 & ๑. . 0000000 & 0.0000000 & ๑. . 0000000 & 0.32 & 0.575 \\
\hline clean & 1 & 0.0000000 & 0.0000000 & 0.0000000 & 0.47 & 0.500 \\
\hline Stackup *Bak & кe1 & 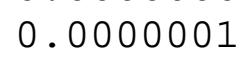 & $\odot .00 \odot \odot \odot \odot 1$ & ๑. . $0 \odot \odot \odot \odot ० 1$ & 1.42 & 0.248 \\
\hline Stackup ${ }^{*} \mathrm{Cln}$ & 1 & 0.0000000 & 0.0000000 & 0.0000000 & 0.09 & 0.766 \\
\hline Bake*Cln & 1 & 0.0000001 & 0.0000001 & 0.0000001 & 1.75 & 0.200 \\
\hline Error & 20 & ๑. . 0000009 & 0.0000009 & ๑. . 0000000 & & \\
\hline Total & 31 & 0.0000013 & & & & \\
\hline
\end{tabular}

$S=0.000210906 \quad R-S q=32.22 \% \quad R-S q(\operatorname{adj})=0.00 \%$ 


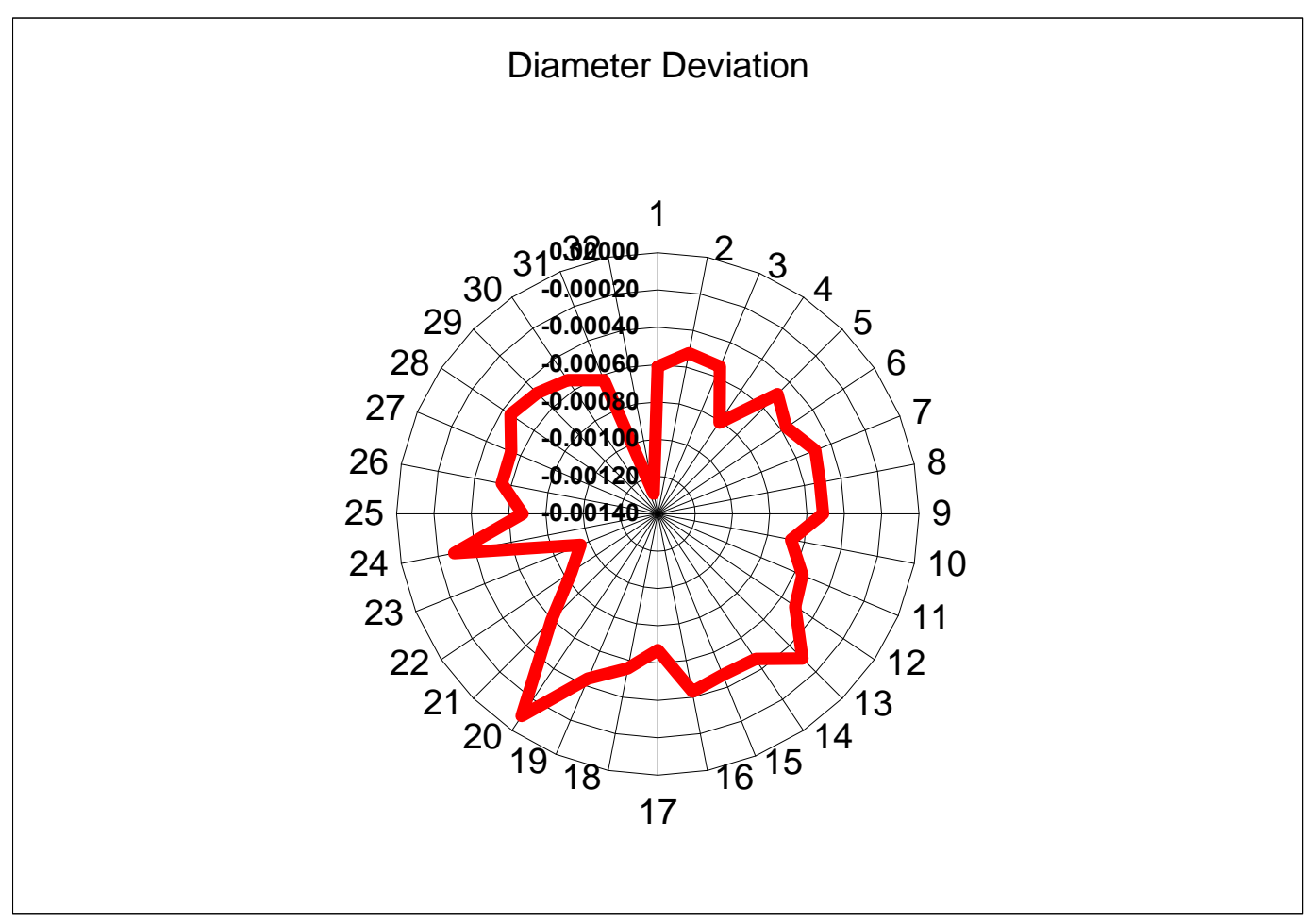

Figure 6. Diameter Deviation

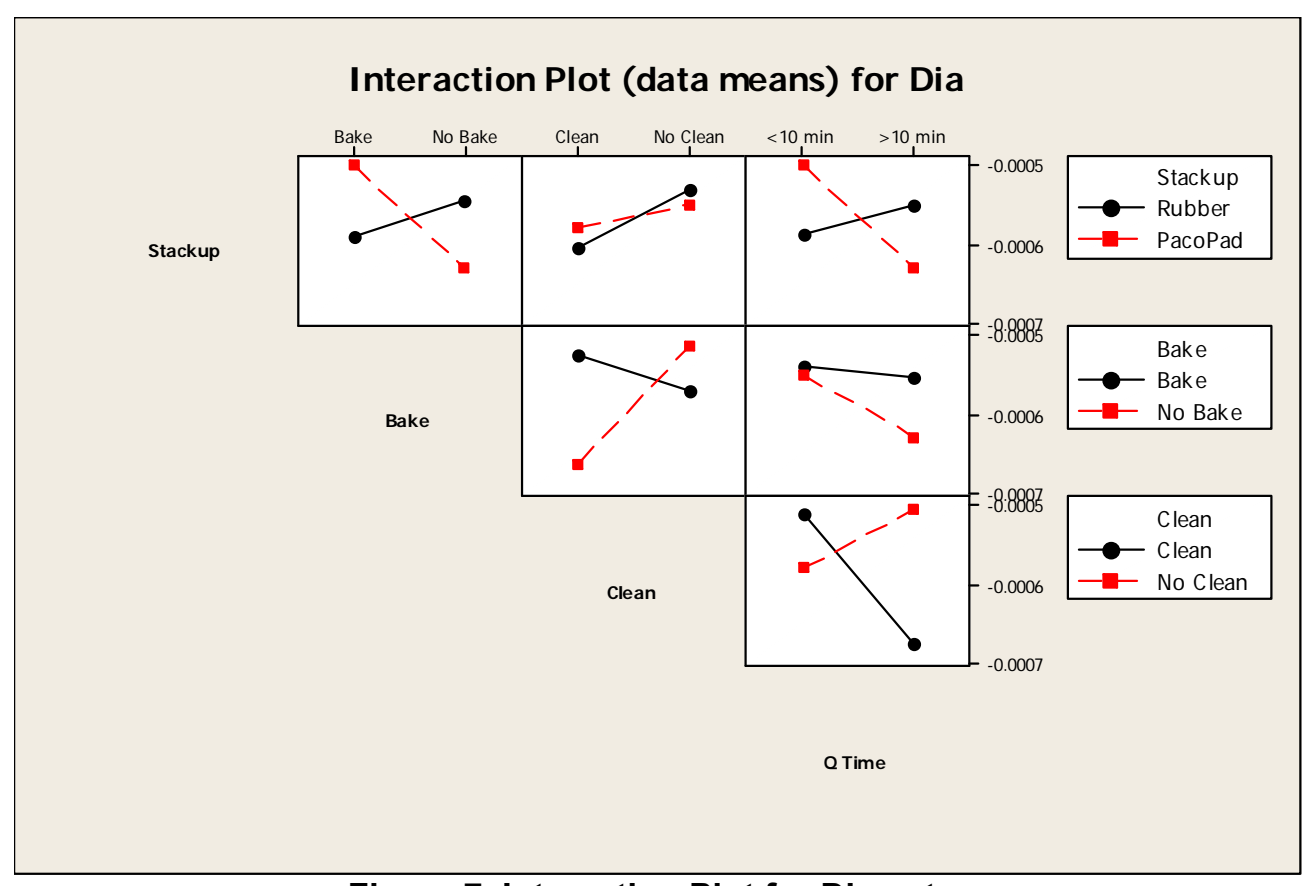

Figure 7. Interaction Plot for Diameter 


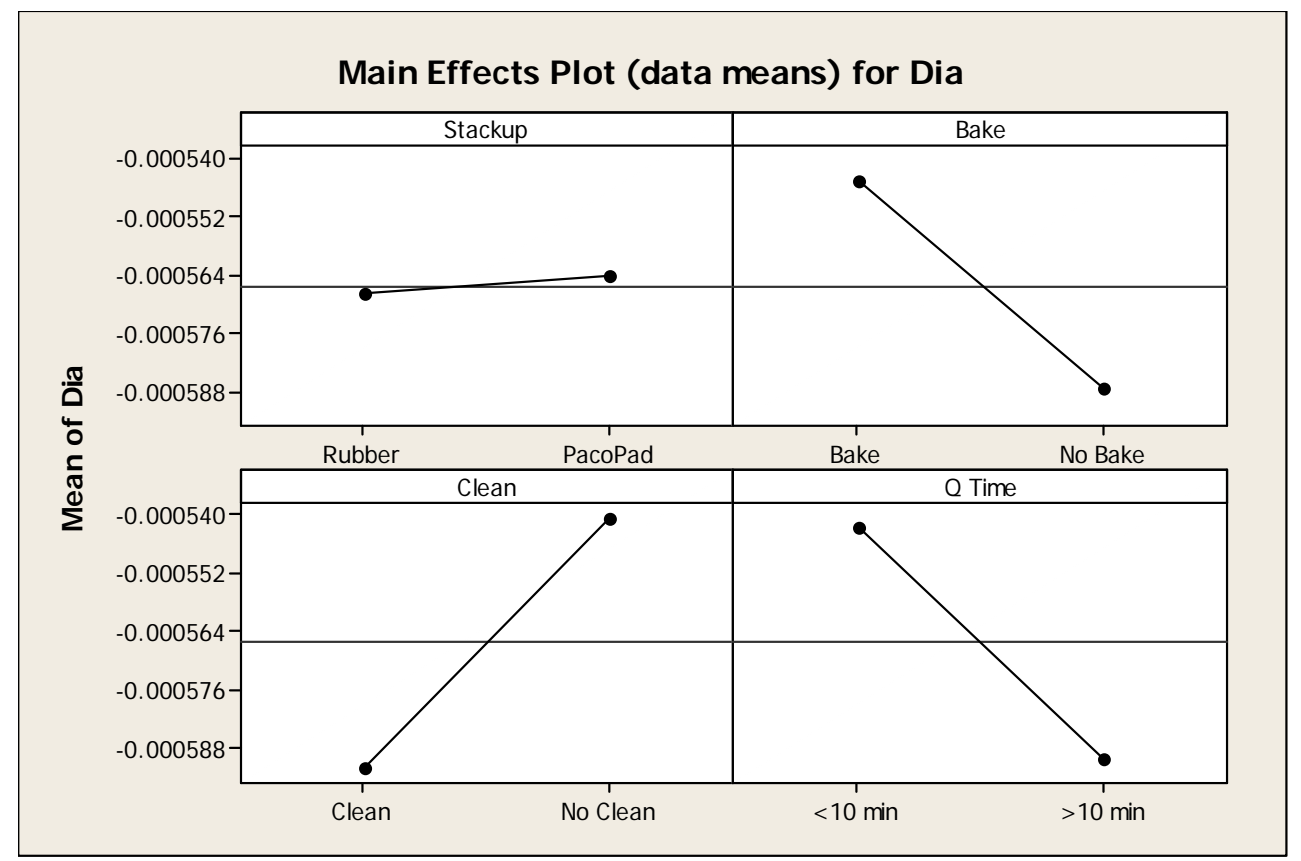

Figure 8. Main Effects Plot for Diameter

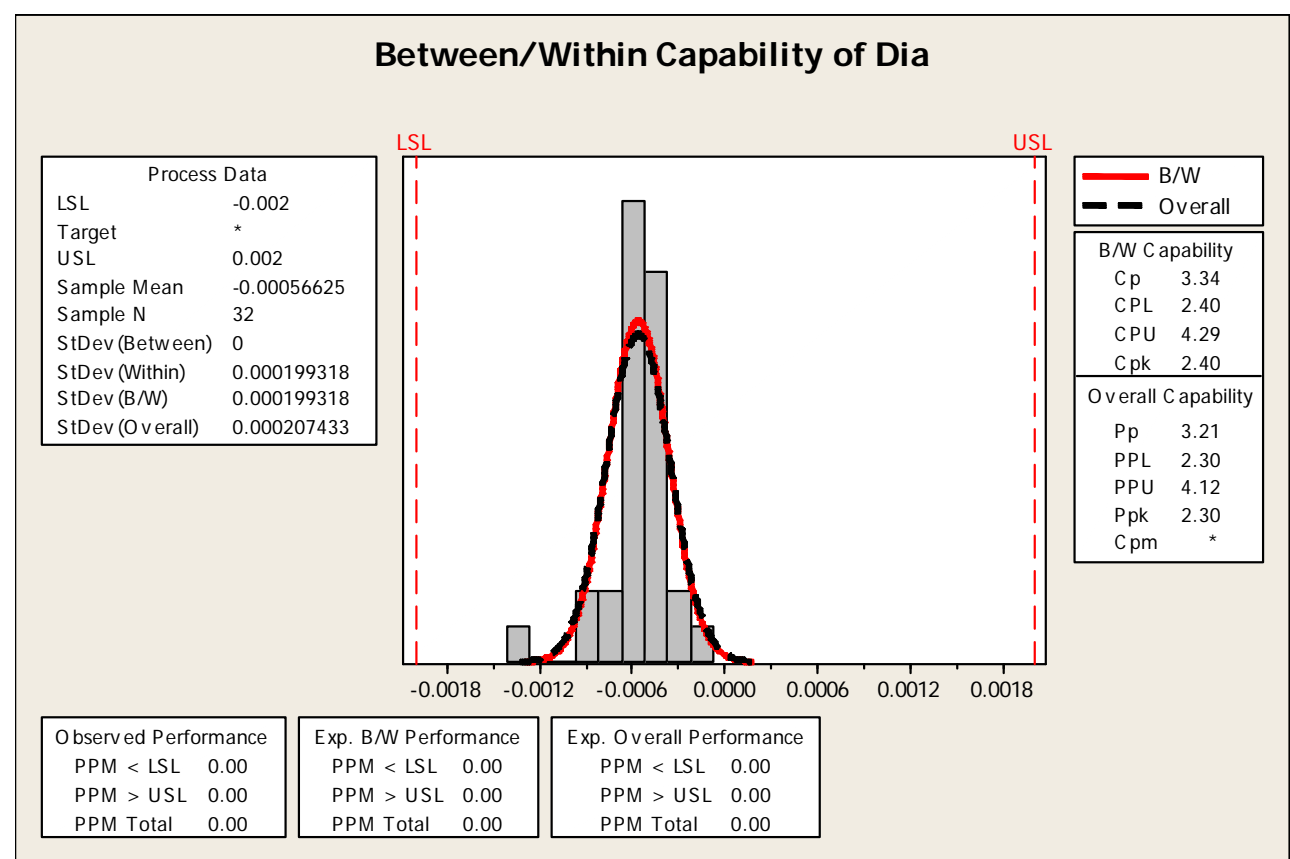

Figure 9. Between/Within Capability of Diameter

\section{Panel Movement in the X Axis}

The panels were next measured for stretch in the $\mathrm{X}$ axis using the CMM. Again $\mathrm{P}$ values less than .1 are statistically significant. In this case the lamination stackup and lamination stackup / clean interaction are both significant. However, the mean stretch in the X axis was only -50.44 ppm and not significant for either press pad stackup. 
Analysis of Variance for X Axis, using Adjusted SS for Tests

$\begin{array}{lrrrrrr}\text { Source } & \text { DF } & \text { Seq SS } & \text { Adj SS } & \text { Adj MS } & \text { F } & P \\ \text { Blocks } & 1 & 108408 & 108408 & 108408 & 5.89 & 0.025 \\ \text { Stackup } & 1 & 195947 & 195947 & 195947 & 10.64 & 0.004 \\ \text { Bake } & 1 & 4687 & 4687 & 4687 & 0.25 & 0.619 \\ \text { Clean } & 1 & 32037 & 32037 & 32037 & 1.74 & 0.202 \\ \text { Stackup*Bake } & 1 & 3069 & 3069 & 3069 & 0.17 & 0.687 \\ \text { Stackup*Clean } & 1 & 88274 & 88274 & 88274 & 4.79 & 0.041 \\ \text { Bake*Clean } & 1 & 37090 & 37090 & 37090 & 2.01 & 0.171 \\ \text { Error } & 20 & 368419 & 368419 & 18421 & & \\ \text { Total } & 31 & 873203 & & & & \end{array}$

$S=135.724 \quad R-S q=57.81 \% \quad R-S q(\operatorname{adj})=34.60 \%$

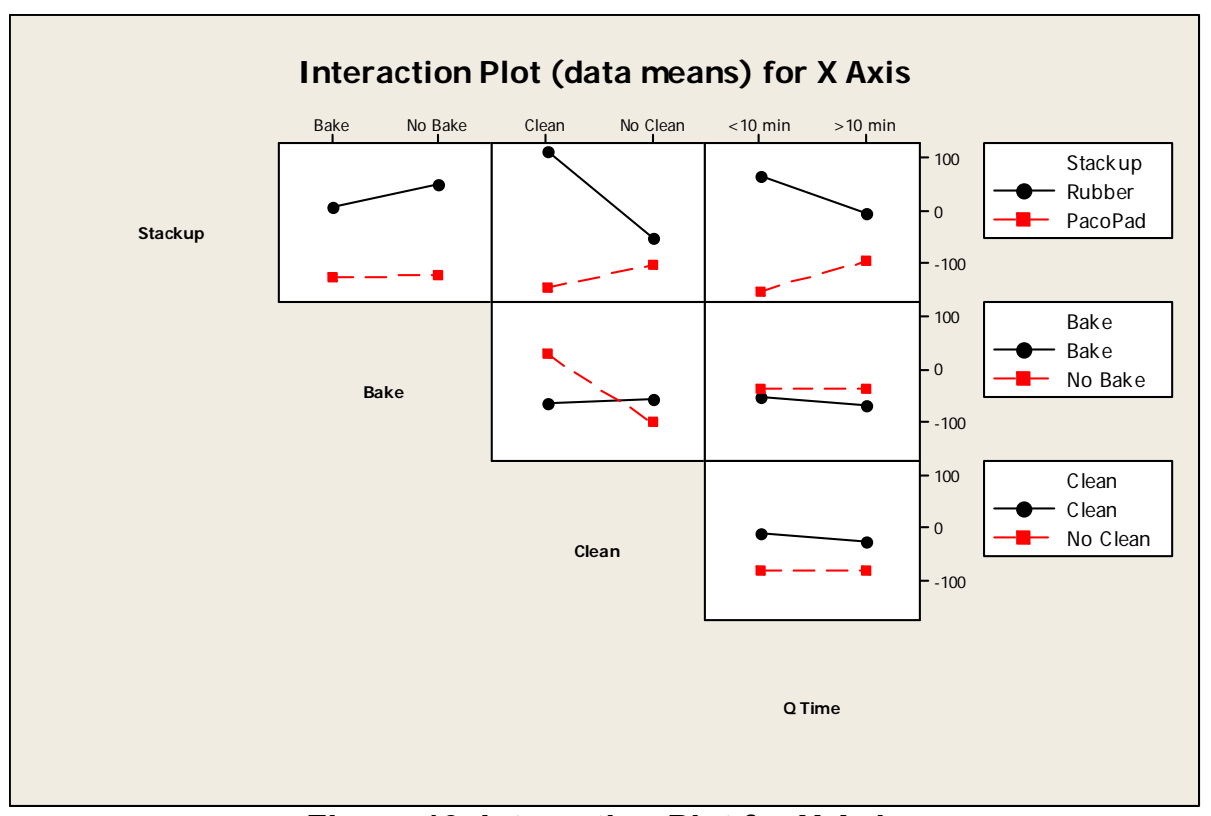

Figure 10. Interaction Plot for X Axis 


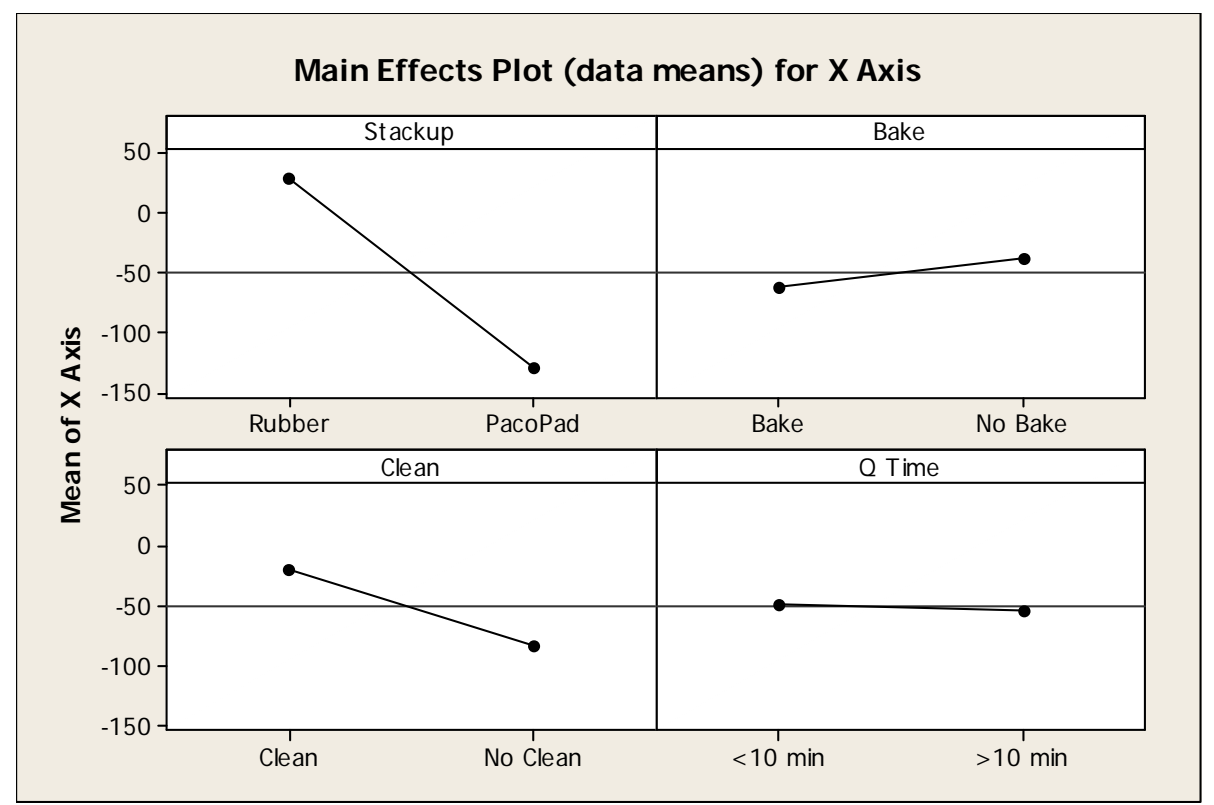

Figure 11. Main Effects Plot for X Axis

\section{Panel Movement in the $\mathbf{Y}$ Axis}

The panels were next measured for stretch in the $\mathrm{Y}$ axis using the CMM. Again $\mathrm{P}$ values less than .1 are statistically significant.

Analysis of Variance for Y Axis, using Adjusted Ss for Tests

$\begin{array}{lrrrrrr}\text { Source } & \text { DF } & \text { Seq SS } & \text { Adj SS } & \text { Adj MS } & \text { F } & \text { P } \\ \text { Blocks } & 1 & 443991 & 443991 & 443991 & 4.29 & 0.052 \\ \text { Stackup } & 1 & 27366663 & 27366663 & 27366663 & 264.16 & 0.000 \\ \text { Bake } & 1 & 236027 & 236027 & 236027 & 2.28 & 0.147 \\ \text { Clean } & 1 & 259317 & 259317 & 259317 & 2.50 & 0.129 \\ \text { Stackup*Bake } & 1 & 112776 & 112776 & 112776 & 1.09 & 0.309 \\ \text { Stackup*Clean1 } & 427140 & 427140 & 427140 & 4.12 & 0.056 \\ \text { Bake*Clean } & 1 & 229525 & 229525 & 229525 & 2.22 & 0.152 \\ \text { Error } & 20 & 2071965 & 2071965 & 103598 & & \\ \text { Total } & 31 & 31343918 & & & & \end{array}$

$S=321.867 \quad R-S q=93.39 \% \quad R-S q(\operatorname{adj})=89.75 \%$

As with the $\mathrm{X}$ axis the lamination stackup and lamination stackup/clean interaction are statistically significant. The total average stretch in the $\mathrm{Y}$ axis was $787.43 \mathrm{ppm}$. The main effects plots in Figure 13 show the large differences in stretch between the soft press pad stackup vs. the hard press pad stackup. The relationship between the stretch in $\mathrm{X}$ and $\mathrm{Y}$ is shown in Figure 14. The two populations represent the two press pad stackups. The average stretch for the hard press pad stackup was $X=27.81$ and $Y=1712.20$ and the soft press pad stackup $X=-128.7$ and $\mathrm{Y}=-137.35$ 


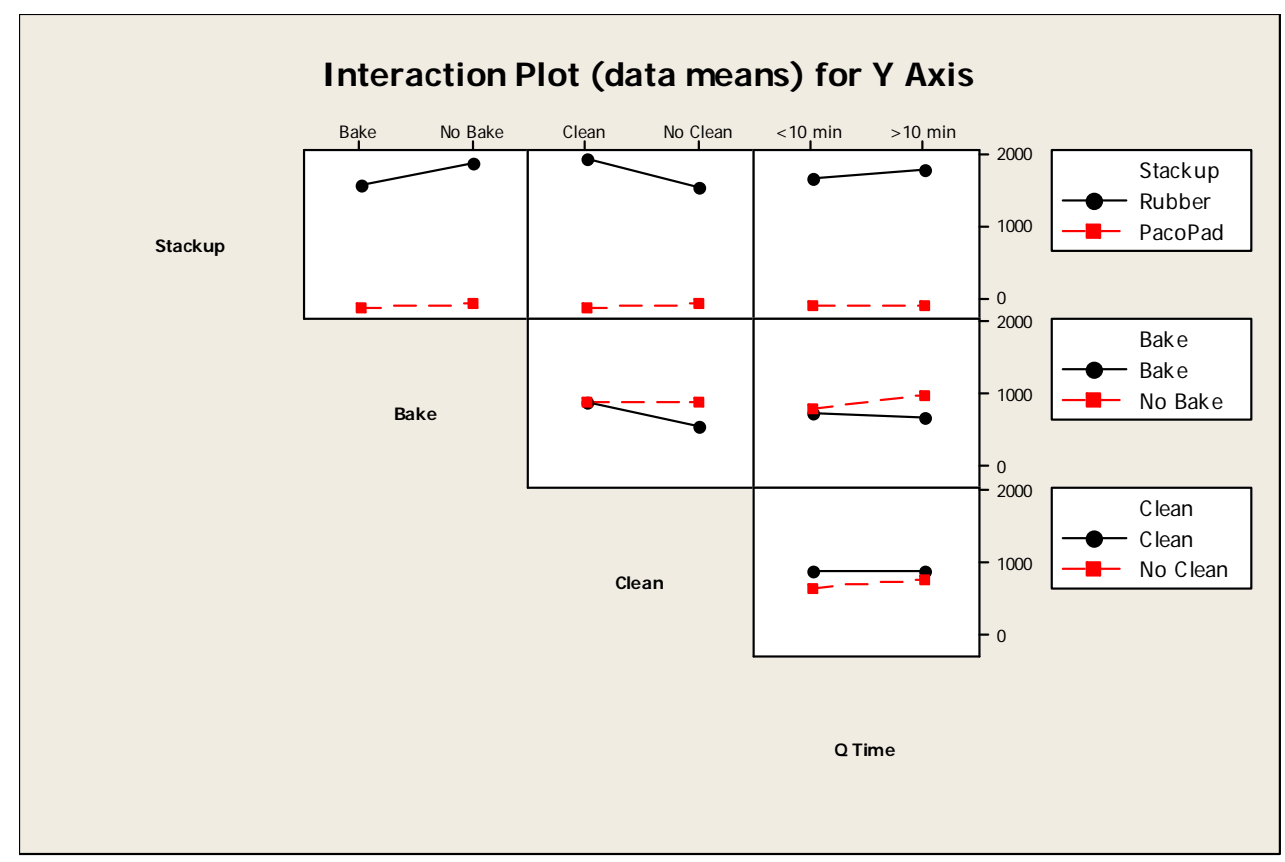

Figure 12. Interaction Plot for $\mathrm{Y}$ Axis

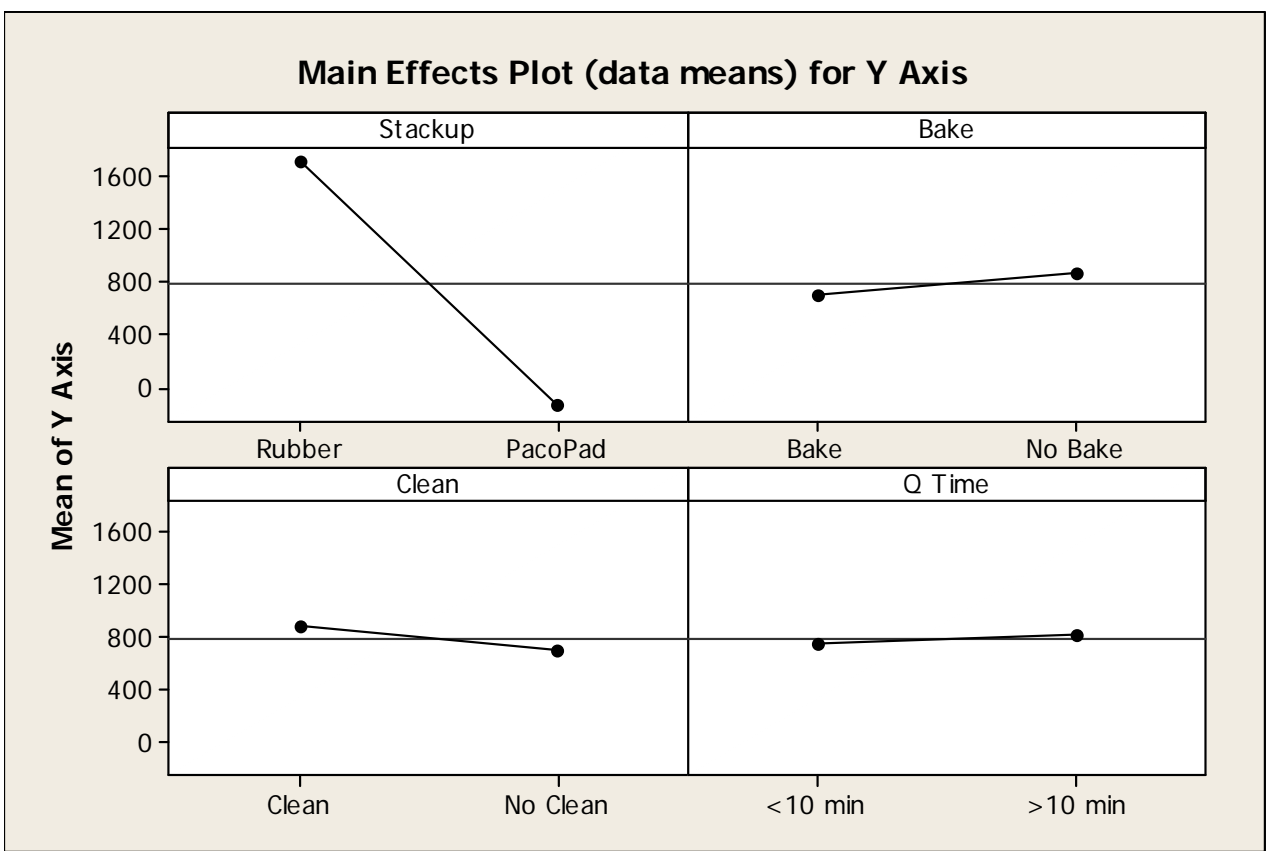

Figure 13. Main Effects Plot for Y Axis 


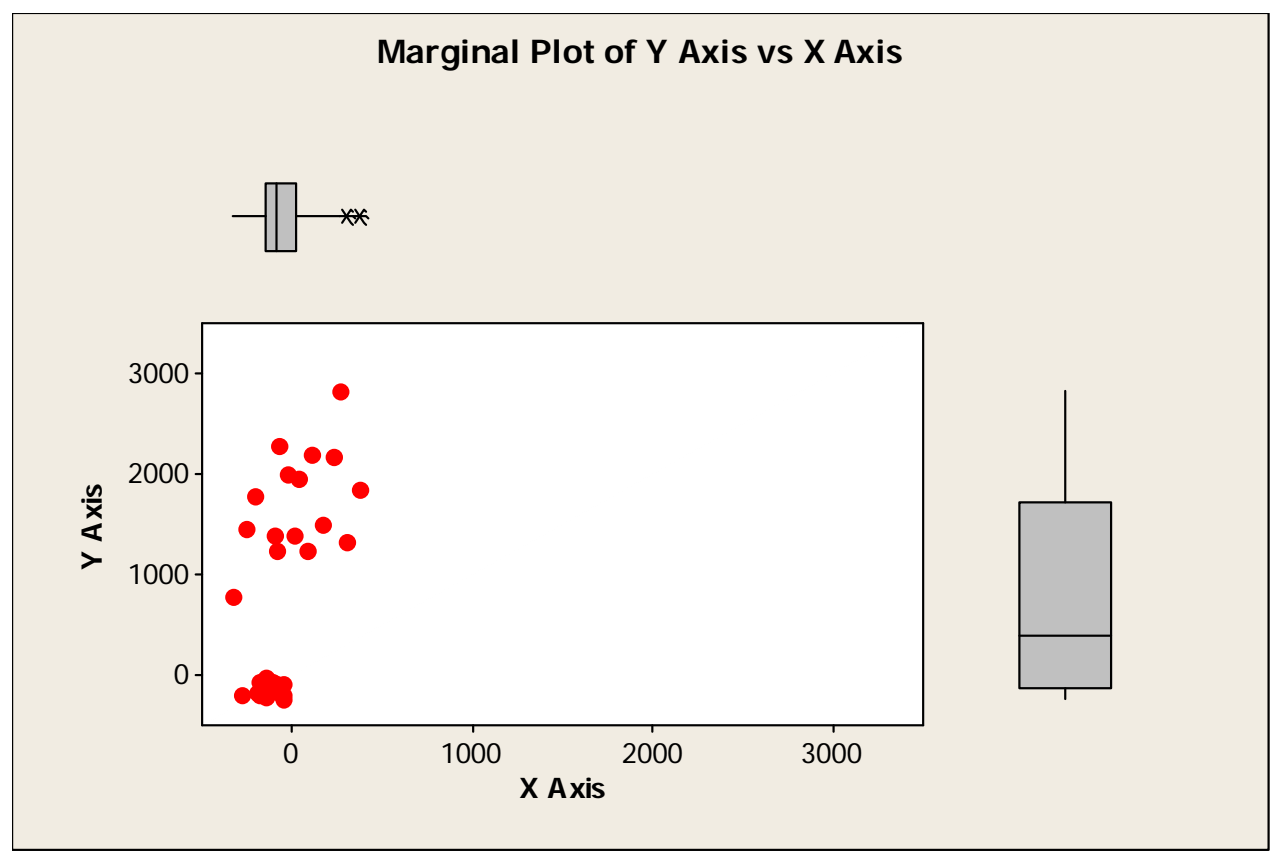

Figure 14. Marginal Plot of $Y$ Axis vs. $X$ Axis

\section{Validation of Phase One Data and Analysis}

Prior to incorporation of process changes suggested by Phase One, the conclusions were validated by performing the experiment a second time with half the number of panels. Two panels/four parts were processed for each variable factor combination resulting in a total of sixteen panels/thirty-two parts. 


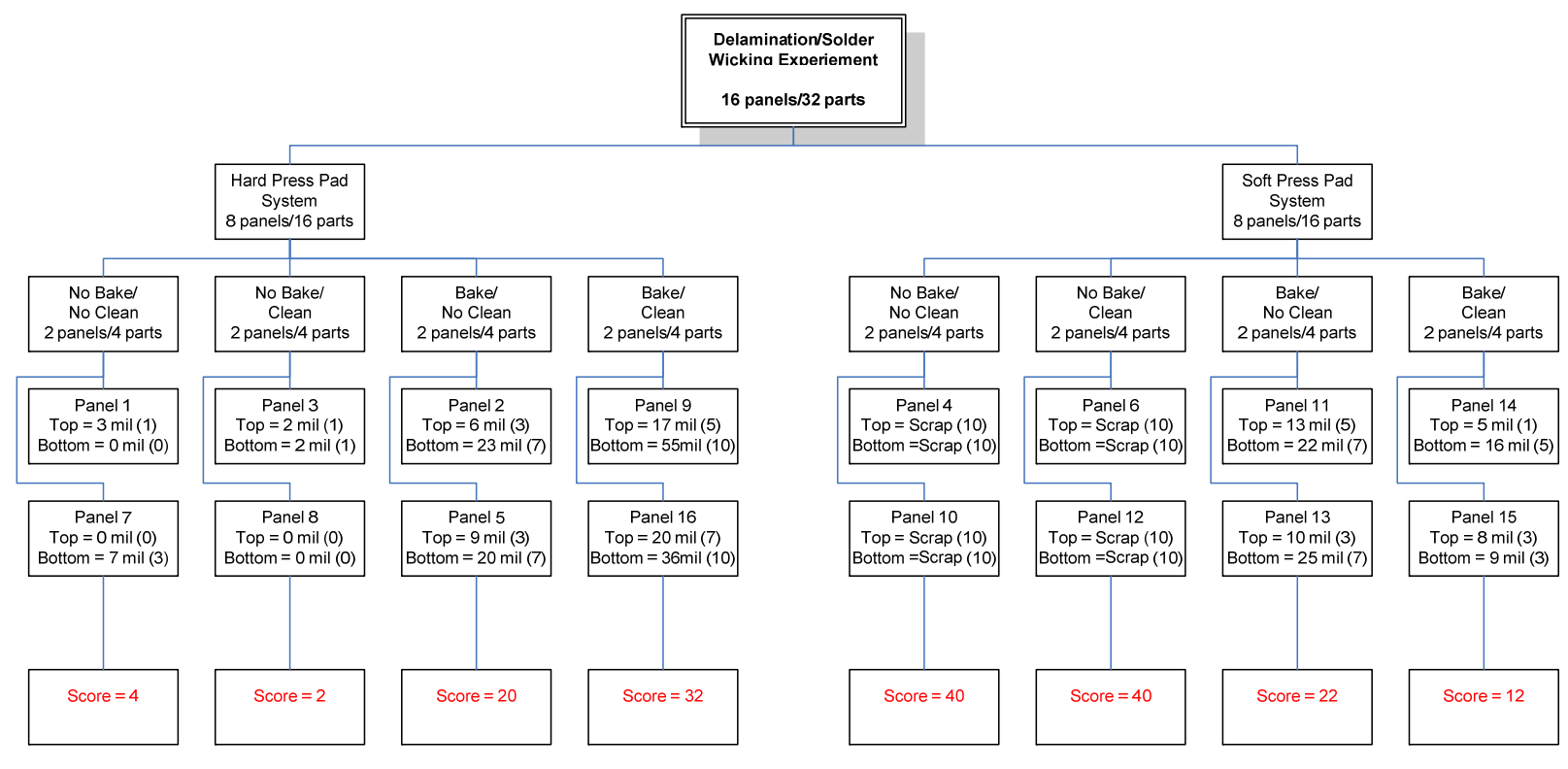

\begin{tabular}{|c|c|}
\hline Hard Press Pad System & \\
\hline No bake/no clean & 4 \\
\hline No bake/clean & 2 \\
\hline Bake/no clean & 20 \\
\hline Bake/clean & 32 \\
\hline Soft Press Pad System & \\
\hline No bake/no clean & 40 \\
\hline No bake/clean & 40 \\
\hline Bake/no clean & 22 \\
\hline Bake/clean & 12 \\
\hline
\end{tabular}

Figure 15. Variable Ranking of Validation Data

The validation data repeated the results from the initial experiment. The hard press pad stackup sample panels not baked or cleaned before HASL had the least amount of discoloration/solder wicking. The difference between baked and not baked was more pronounced on these hard press pad stackup sample panels. The soft press pad stackup sample panels had significant solder wicking on the panels not baked and cleaned/not cleaned. 
Original DoE Results

32 panels/64 cables

\begin{tabular}{||ll||}
\hline Hard Press Pad System & Score \\
No bake/no clean & 12 \\
No bake/clean & 18 \\
Bake/no clean & 23 \\
Bake/clean & 58 \\
& \\
Soft Press Pad System & \\
No bake/no clean & 80 \\
No bake/clean & 80 \\
Bake/no clean & 40 \\
Bake/clean & 36 \\
\hline
\end{tabular}

Validation DoE Results

16 panels/32 cables

\begin{tabular}{|c|c|}
\hline Hard Press Pad System & Score \\
\hline No bake/no clean & 4 \\
\hline No bake/clean & 2 \\
\hline Bake/no clean & 20 \\
\hline Bake/clean & 32 \\
\hline Soft Press Pad System & \\
\hline No bake/no clean & 40 \\
\hline No bake/clean & 40 \\
\hline Bake/no clean & 22 \\
\hline Bake/clean & 12 \\
\hline
\end{tabular}

\begin{tabular}{|c|c|}
\hline Hard Press Pad System & \\
\hline No bake/no clean & 16 \\
\hline No bake/clean & 20 \\
\hline Bake/no clean & 43 \\
\hline Bake/clean & 90 \\
\hline Soft Press Pad System & \\
\hline No bake/no clean & 120 \\
\hline No bake/clean & 120 \\
\hline Bake/no clean & 62 \\
\hline Bake/clean & 48 \\
\hline
\end{tabular}

Combined DoE Results 48 panels/96 cables

Figure 16. Original, Validation and Combined Variable Ranking

\section{Conclusions}

Performing the DoE utilizing the most likely variables did not recreate the large voids, but did illustrate other possible processing modifications of interest.

The hard press pad stackup obtained the best results or the least amount of discoloration/solder wicking with variable factors of "no bake/no clean." This also was the combination of variable factors exhibiting the best adhesion of all variables for both press pad stackups. None of the "bake/clean" results were as good. Thirteen of the twenty-four cables had no observable characteristics for a $54 \%$ yield.

The soft press pad stackup obtained the best results with variable factors "bake/clean" and "bake/no clean." Variable factor "bake/clean" performed slightly better then variable factor "bake/no clean." All twenty-four cables not baked, cleaned or not cleaned, had significant amounts of solder wicking under the coverlay. This combination of variables implied inadequate adhesion. 
The baking and cleaning parameters had no significant effect on hole diameter. Any stretch in the $\mathrm{X}$ direction was insignificant for all combinations. Stretch in the Y direction using the hard press pad stackup was significantly more than the soft press pad stackup. The average X and Y stretch by press pad stackup is shown below.

\begin{tabular}{|l|l|l|}
\hline & $\begin{array}{l}\text { Average X Stretch } \\
(\mathrm{ppm})\end{array}$ & $\begin{array}{l}\text { Average Y Stretch } \\
(\mathrm{ppm})\end{array}$ \\
\hline Hard Press Pad Stackup & 27.81 & 1712.20 \\
\hline Soft Press Pad Stackup & -128.70 & -137.35 \\
\hline
\end{tabular}

\section{Phase Two}

This DoE's objective was to determine if a lower lamination pressure would reduce panel stretch without adversely affecting coverlay/laminate adhesion when using the hard press pad stackup.

\section{Sample}

A twelve-by-twelve inch, two ounce copper, double-sided laminate sample was generated which contained the circuit patterns of five cables. The coverlay laminated to both sides of the laminate samples was two mil polyimide/two mil pyralux adhesive. Nine holes with a diameter of .060 inch were strategically located on the sample panel for CMM hole location measurements.

\section{Experiment}

A full factorial experiment with one independent variable, pressure, was developed. Three pressure values were selected with the 190 ton factor the standard for presently built flex cables.

\begin{tabular}{|l|l|l|l|}
\hline Variable & Level & Level & Level \\
\hline Pressure & 190 ton & 140 ton & 90 ton \\
\hline
\end{tabular}

Three sample panels were processed at each lamination pressure. All were laminated in the Technical Machine Products (TMP) lamination press over a period of three days. Each lamination cycle's actual temperature, pressure, and soak time were recorded at one minute intervals. After lamination each panel's nine holes were measured for location by a Department's CMM and recorded.

It has been acknowledged that the HASL process is an acceptable test of laminated adhesion quality. To determine the acceptability of adhesion of each group of sample panels they were processed through the normal HASL process after lamination.

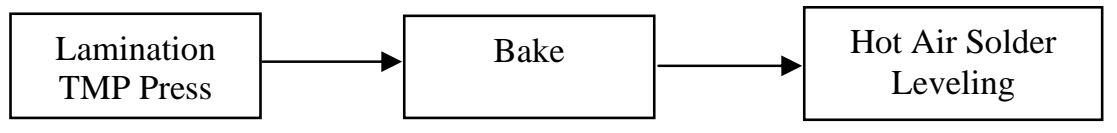

There have been some indications suggesting discoloration around lands is a precursor to delamination. Therefore, measuring the depth of solder wicking/discoloration on each land of all 
five cables per panel was determined to be an acceptable method of quantifying adhesion quality. The greater the solder wicking/discoloration the more likely there is inadequate adhesion. The total number of lands per panel was fifty-nine.

\section{Hole Location Analysis}

The chart below illustrates Parts Per Million (PPM) stretch of sample panels in both the $\mathrm{x}$ and $\mathrm{y}$ axis. Sample panels 1, 2, and 3 were laminated at 190 tons, sample panels 4, 5, and 6 were laminated at 140 tons and sample panels 7, 8, and 9 were laminated at 90 tons. The nine .060 inch hole location measurements were used to generate the chart.

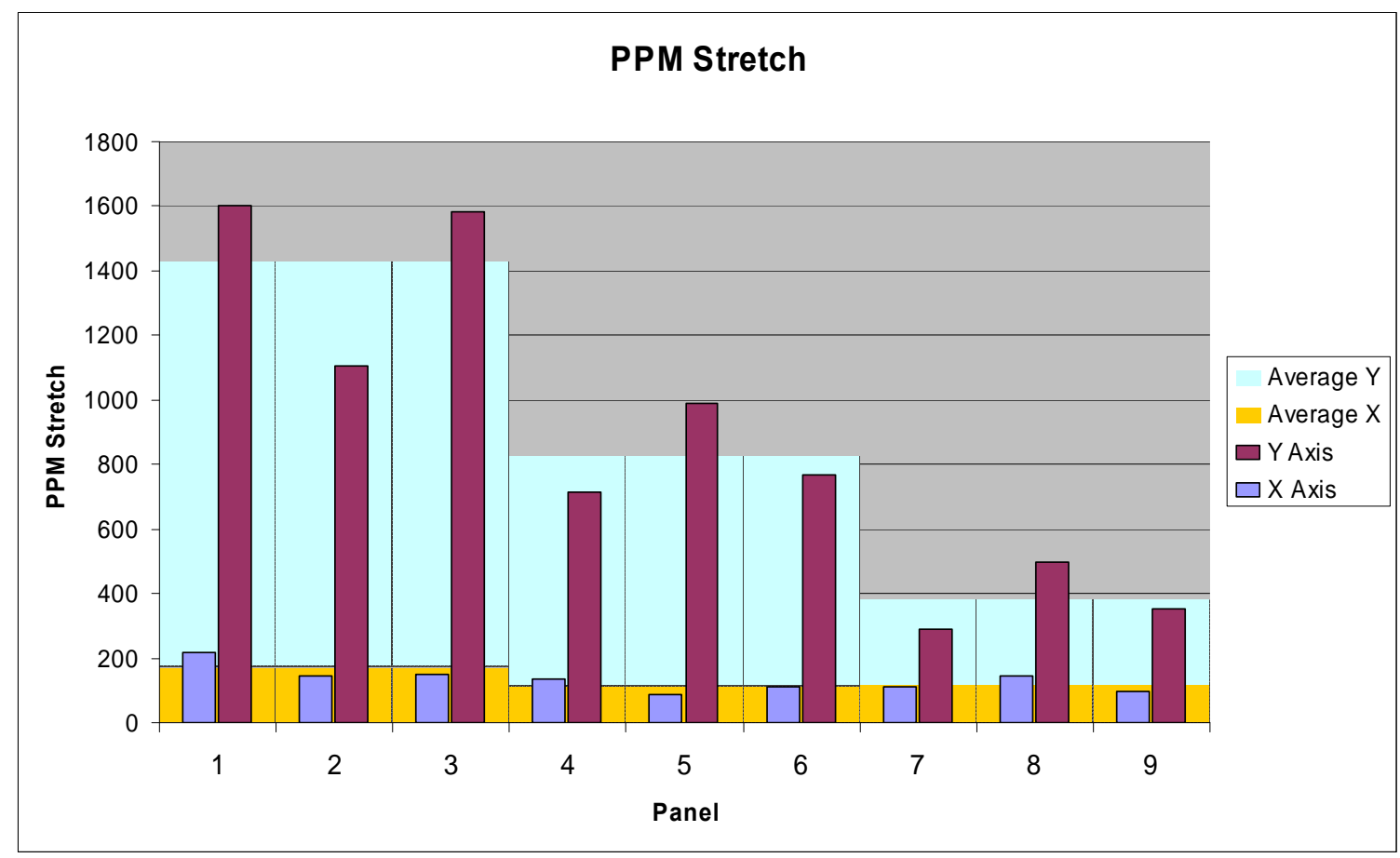

Figure 17. Parts Per Million Stretch of Sample Panels 


\section{Adhesion Quality Analysis}

Each part was given a ranking using a scoring technique similar to the method used on Phase 1 panels. The illustration below summarizes each part per panel ranking and each panels ranking as a sum of each parts ranking. The panel rankings are then summed to provide an overall lamination pressure score.

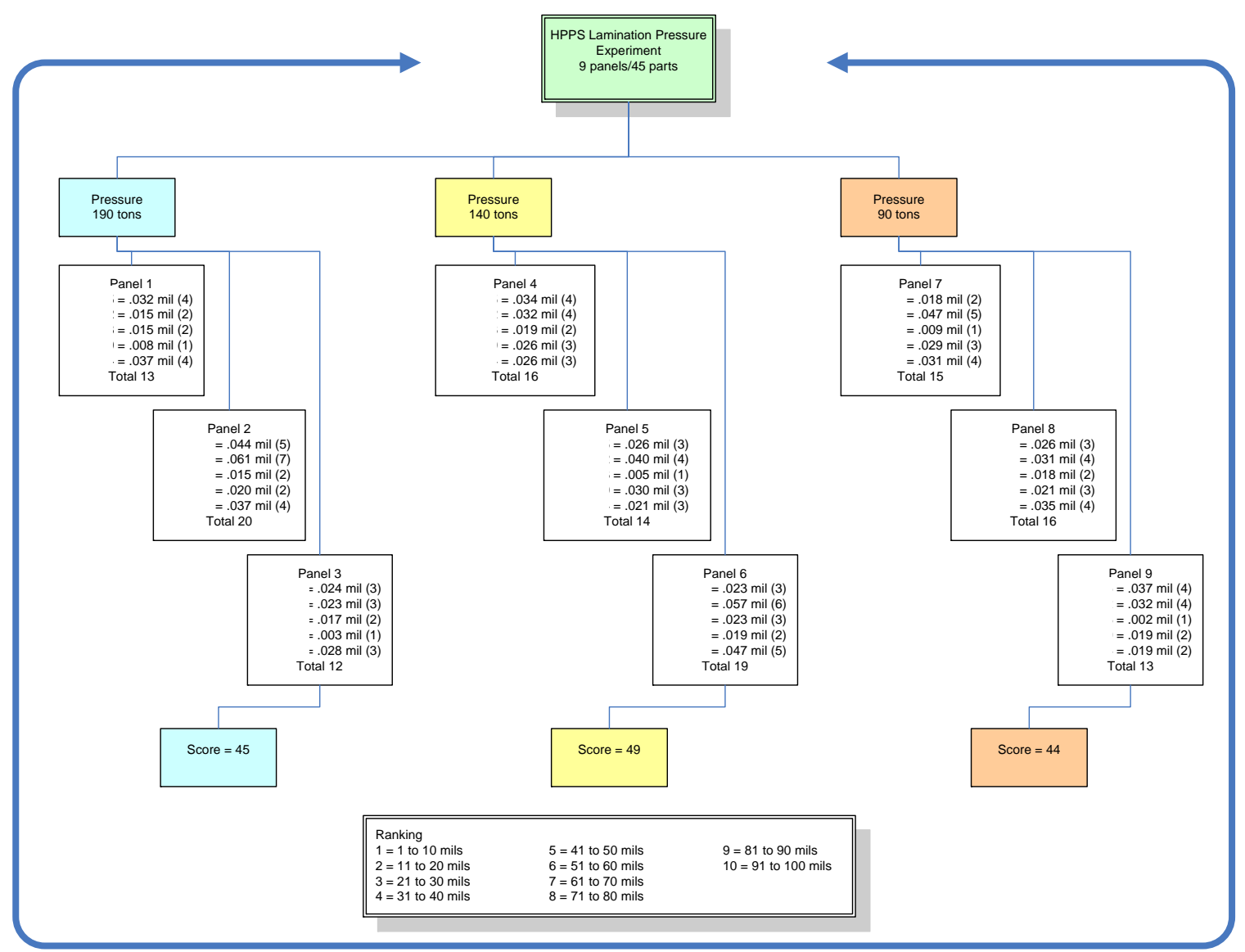

Figure 18. Panel Ranking

\section{Conclusions}

A reduction in lamination pressure from 190 tons to 90 tons reduced panel stretch in the y direction by approximately $70 \%$. Panel stretch was small in the $\mathrm{x}$ direction and was only slightly affected by a pressure change. The reduction in panel stretch could reduce or eliminate further out of tolerance occurrences on the hole location requirements for flat flex cables.

The adhesion quality results do not illustrate any lack of adhesion when using a lower lamination pressure. The differences in ranking scores are statistically insignificant. Based on these results, reducing the lamination pressure from 190 tons to 90 tons should not affect the quality of adhesion between coverlay and copper laminate. 


\section{Phase Three}

This DoE's objective was to determine if variations in lamination and pre-HASL process variables affected delamination/solder wicking around circuit lands when using the soft press pad lamination stackup.

\section{Sample (Two Ounce Copper Laminate)}

A twelve-by-twelve inch, two ounce copper, double-sided laminate sample was generated which contained the circuit patterns of five cables. The coverlay laminated to both sides of the laminate samples was two mil polyimide/two mil pyralux adhesive. Nine holes with a diameter of .060 inch were strategically located on the sample panel for CMM hole location measurements.

\section{Experiment}

A fractional factorial experiment with six independent variables was developed. Two factors were selected for each variable as shown in the table below.

\begin{tabular}{|c|c|c|}
\hline Variable & Level & Level \\
\hline Pressure & $300 \mathrm{psi}$ & $350 \mathrm{psi}$ \\
\hline Time & $60 \mathrm{~min}$ & $80 \mathrm{~min}$ \\
\hline Temperature & $365 \mathrm{~F}$ & $385 \mathrm{~F}$ \\
\hline Bake & $275 \mathrm{~F}$ & $350 \mathrm{~F}$ \\
\hline Bake Time & 1 & 8 \\
\hline Coverlay Adhesive & $2 \mathrm{mil}$ & 4 mil \\
\hline
\end{tabular}

Two sample panels were processed for each variable and factor combination resulting in thirtytwo panels total. All were laminated in the TMP lamination press over a period of five days. Each lamination cycle's actual temperature, pressure, and soak time were recorded at one minute intervals. After lamination each panel's nine holes were measured for location by a Department's CMM and recorded. Below is a process map of lamination through HASL.

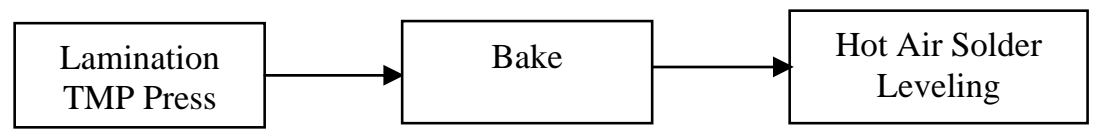

Measuring the delamination/solder wicking depth on each land of all five cables per panel was determined to be an acceptable method of quantifying adhesion quality. The greater the delamination/solder wicking the more likely there is inadequate adhesion. The total number of lands per panel was fifty-nine. 


\section{Hole Location Analysis}

The chart below illustrates parts per million stretch of sample panels in both the $\mathrm{x}$ and $\mathrm{y}$ axis. The .093 inch hole location measurements were used to generate the chart.

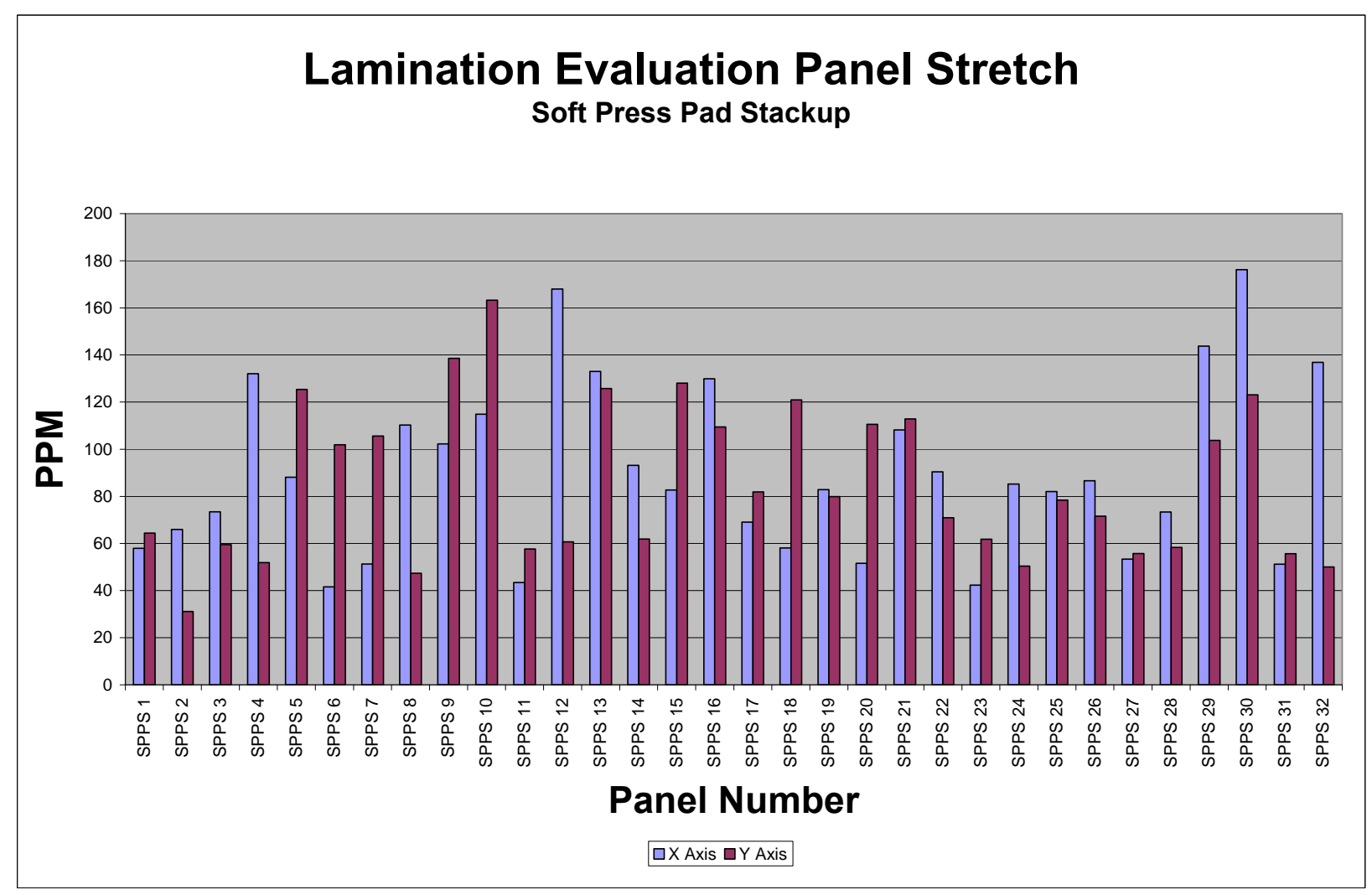

Figure 19. Lamination Evaluation Panel Stretch (Soft Press Pad Stackup) 
Below is a chart comparing panel stretch between Phase Two hard press pad stackup sample panels and Phase Three soft press pad stackup sample panels. The chart illustrates an approximate factor of ten times more panel stretch between the 190 ton pressure used on hard press pad lamination stackup and the same pressure used on the soft press pad lamination stackup. When the hard press pad lamination stackup pressure is reduced to 90 tons, the panel stretch difference between the two press pad stackups is a factor of 2 with the hard press pad stackup having the most panel stretch.

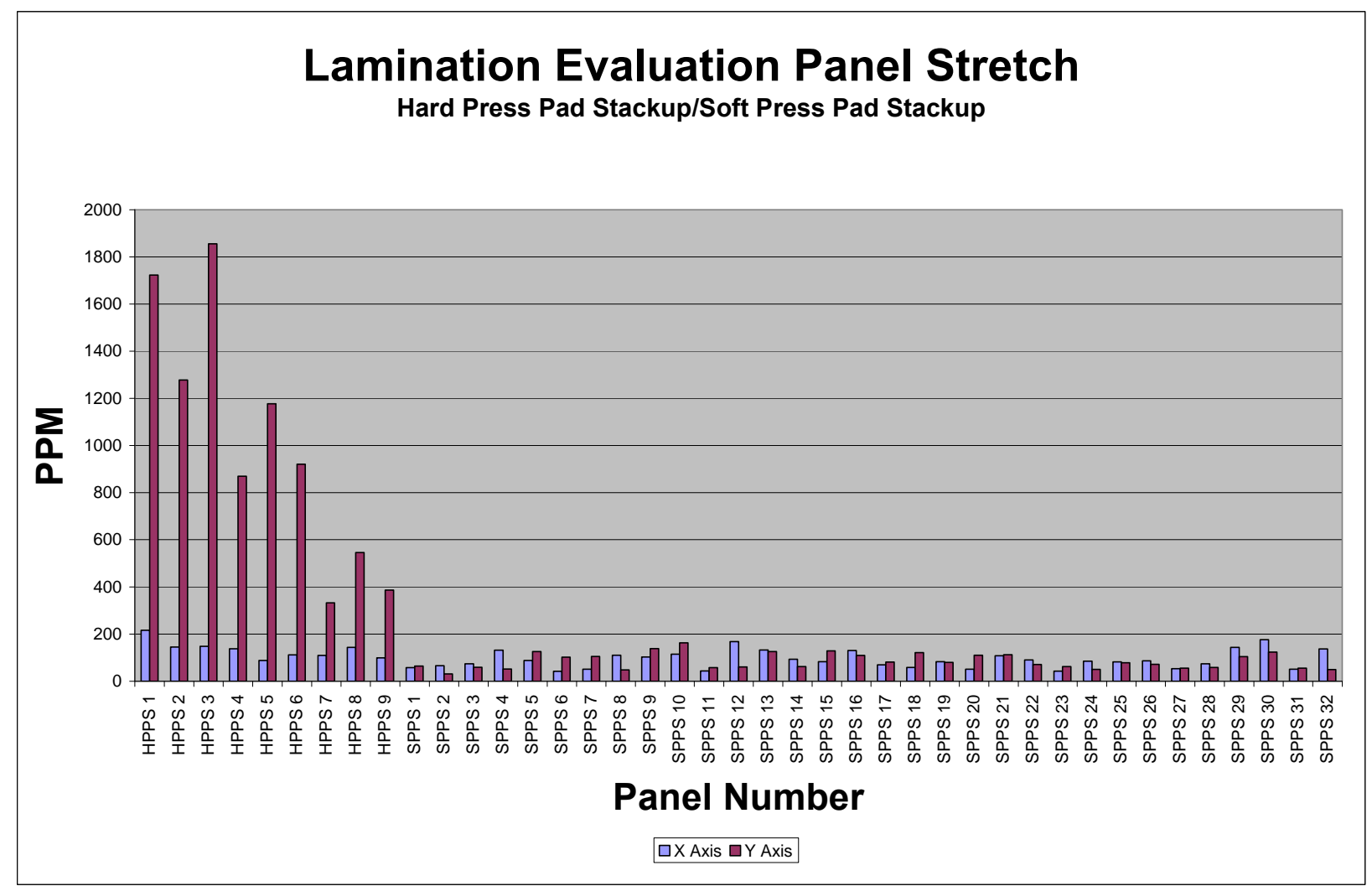

Figure 20. Lamination Evaluation Panel Stretch (Hard Press Pad Stackup/Soft Press Pad Stackup) 


\section{Adhesion Quality Analysis}

The table below summarizes each panels ranking as a sum of each parts ranking. The fill color highlights each pre-HASL bake temperature and time. When comparing solder wicking rank of each colored group highlighted, it is evident a higher pre-HASL bake could eliminate delamination/solder wicking when using a soft press pad stackup. The table below also illustrates how a higher bake temperature will cause a semi dark to very dark 'halo' under the coverlay around each circuit land as illustrated in Figures 23 and 24. See Appendix C for individual panel rankings (magnitude of discoloration/solder wicking).

\begin{tabular}{|c|c|c|c|c|c|c|c|c|}
\hline \multirow[b]{2}{*}{ Panel Number } & \multirow{2}{*}{\begin{tabular}{|c|} 
Extra 2mil \\
Adhesive,Both \\
Sides \\
\end{tabular}} & \multirow[b]{2}{*}{ Bake Temperature } & \multirow[b]{2}{*}{ Bake Time } & \multicolumn{3}{|c|}{ Lamination } & \multirow[b]{2}{*}{$\begin{array}{c}\text { Solder Wicking } \\
\text { Rank }\end{array}$} & \multirow[b]{2}{*}{$\begin{array}{l}\text { Dark Halo/Annular } \\
\text { Ring/Delamination }\end{array}$} \\
\hline & & & & Pressure & Temperature & Time & & \\
\hline 3 & Yes & 275 & 1 & 300 & 385 & 60 & 28 & /ULL/ \\
\hline 12 & Yes & 275 & 1 & 300 & 365 & 80 & 36 & /ULL/ \\
\hline 19 & Yes & 275 & 1 & 300 & 385 & 60 & 20 & /ULL/ \\
\hline 28 & No & 275 & 1 & 300 & 365 & 80 & 40 & \\
\hline 2 & No & 275 & 1 & 350 & 385 & 80 & 28 & \\
\hline 15 & No & 275 & 1 & 350 & 365 & 60 & 45 & \\
\hline 18 & Yes & 275 & 1 & 350 & 385 & 80 & 31 & /ULL/ \\
\hline 31 & Yes & 275 & 1 & 350 & 365 & 60 & 43 & /ULL/ \\
\hline 9 & No & 275 & 8 & 300 & 365 & 60 & 3 & \\
\hline 13 & No & 275 & 8 & 300 & 385 & 80 & 1 & \\
\hline 25 & Yes & 275 & 8 & 300 & 365 & 60 & 3 & /ULL/ \\
\hline 29 & No & 275 & 8 & 300 & 385 & 80 & 3 & \\
\hline 4 & No & 275 & 8 & 350 & 385 & 60 & 2 & \\
\hline 7 & Yes & 275 & 8 & 350 & 365 & 80 & 1 & /ULL/ \\
\hline 20 & Yes & 275 & 8 & 350 & 385 & 60 & 2 & /ULL/ \\
\hline 23 & Yes & 275 & 8 & 350 & 365 & 80 & 4 & /ULL/ \\
\hline 1 & Yes & 350 & 1 & 300 & 385 & 80 & 0 & .005 light/ULL/ \\
\hline 10 & No & 350 & 1 & 300 & 365 & 60 & 0 & .005 light// \\
\hline 17 & Yes & 350 & 1 & 300 & 385 & 80 & 0 & .005 light/ULL/ \\
\hline 26 & Yes & 350 & 1 & 300 & 365 & 60 & 0 & .005 light/ULL/ \\
\hline 6 & No & 350 & 1 & 350 & 385 & 60 & 0 & .005 light// \\
\hline 8 & No & 350 & 1 & 350 & 365 & 80 & 0 & .005 light// \\
\hline 22 & Yes & 350 & 1 & 350 & 385 & 60 & 0 & .005 light/ULL/ \\
\hline 24 & No & 350 & 1 & 350 & 365 & 80 & 0 & .005 light// \\
\hline 5 & No & 350 & 8 & 300 & 365 & 80 & 0 & 0.010 dark $/ /$ \\
\hline 16 & No & 350 & 8 & 300 & 385 & 60 & 0 & 0.010 dark// \\
\hline 21 & Yes & 350 & 8 & 300 & 365 & 80 & 0 & 0.010 dark/ULL/Light spots \\
\hline 32 & No & 350 & 8 & 300 & 385 & 60 & 0 & 0.010 dark//Light spots \\
\hline 11 & Yes & 350 & 8 & 350 & 385 & 80 & 0 & 0.010 dark/ULL/Light spots \\
\hline 14 & Yes & 350 & 8 & 350 & 365 & 60 & 0 & 0.010 dark/ULL/Light spots \\
\hline 27 & No & 350 & 8 & 350 & 385 & 80 & 0 & 0.010 dark//Light spots \\
\hline 30 & No & 350 & 8 & 350 & 365 & 60 & 0 & 0.010 dark//Light spots \\
\hline
\end{tabular}

Table 1. Panel Ranking 


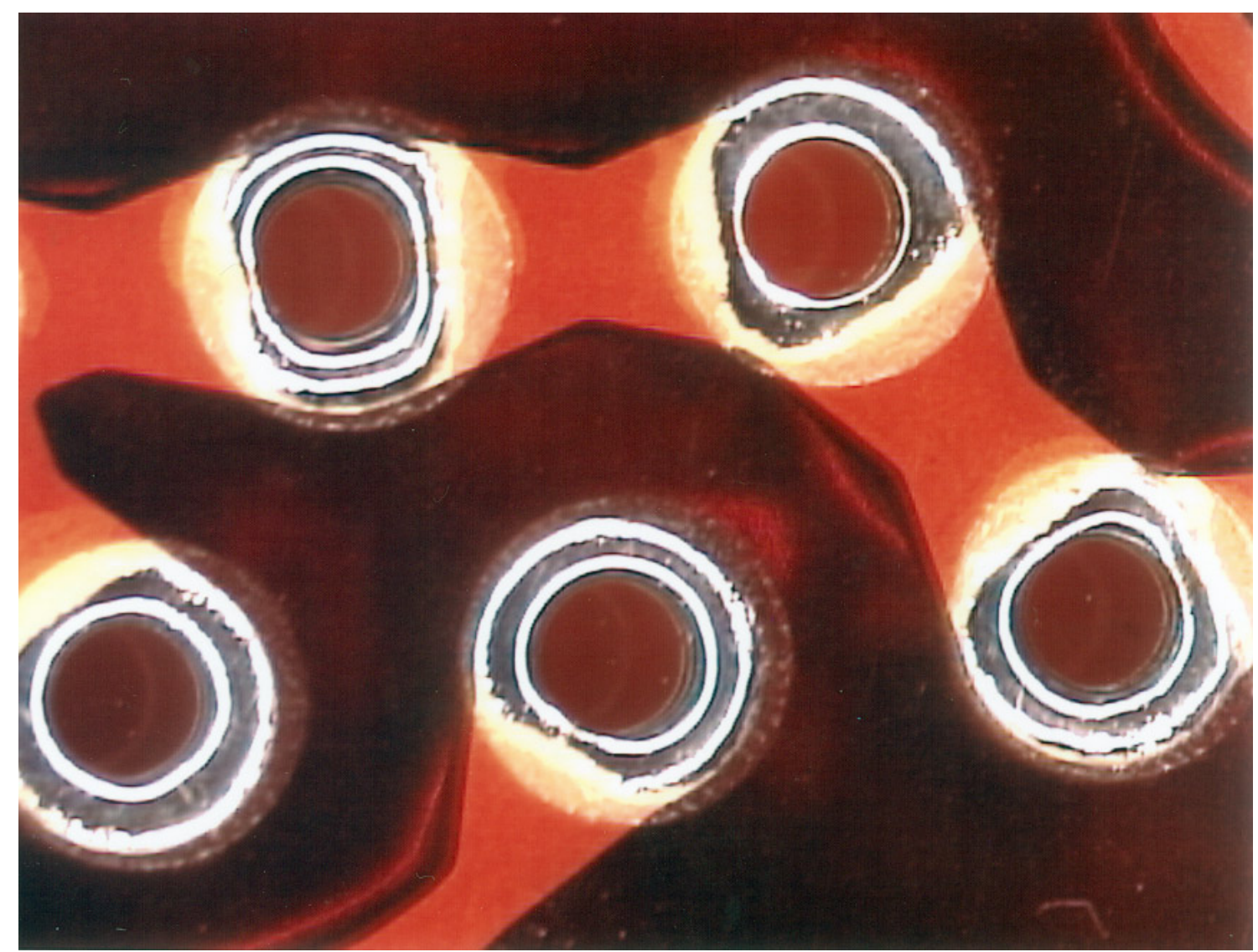

Figure 21. Annular Rings 


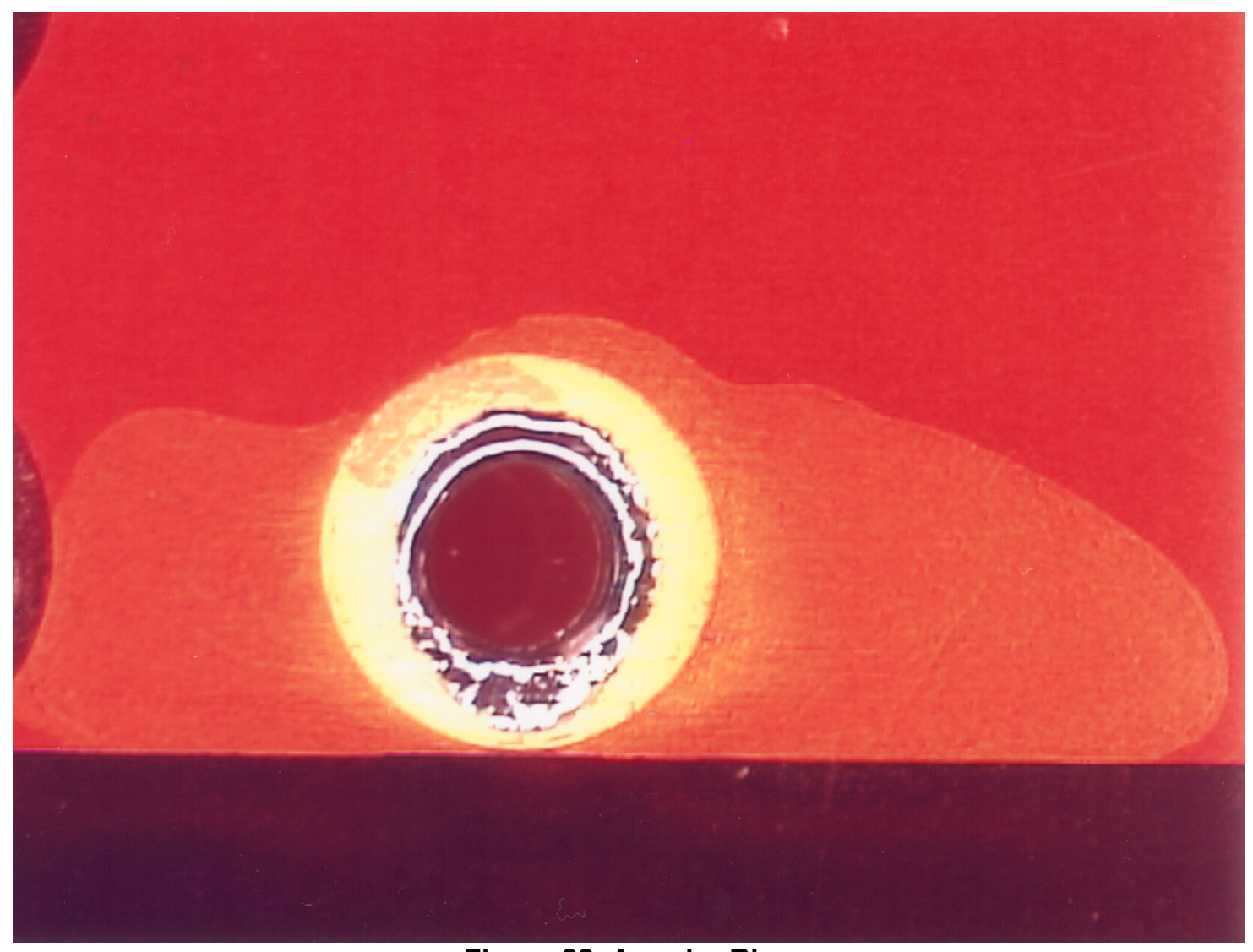

Figure 22. Annular Ring 


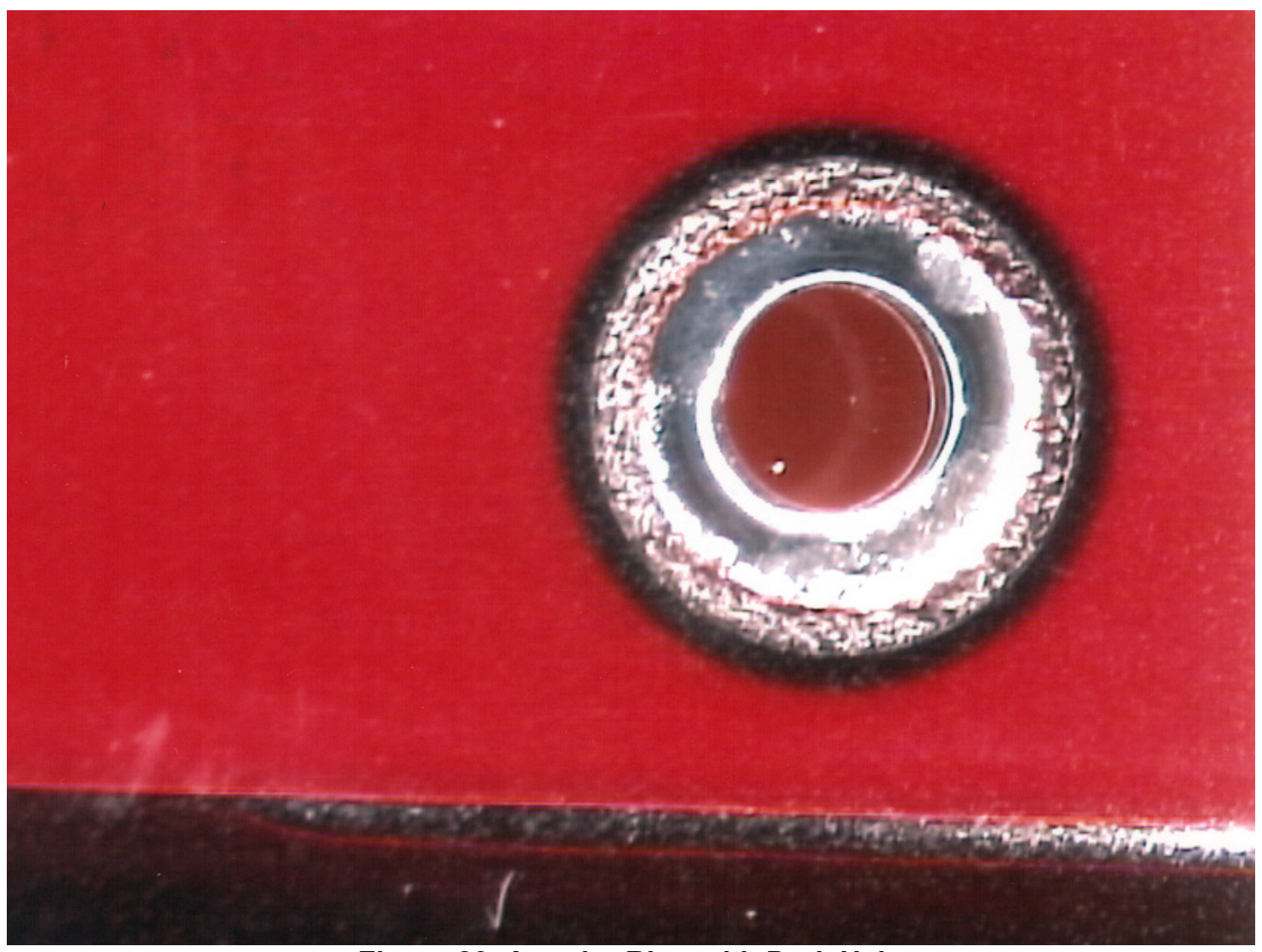

Figure 23. Annular Ring with Dark Halo 


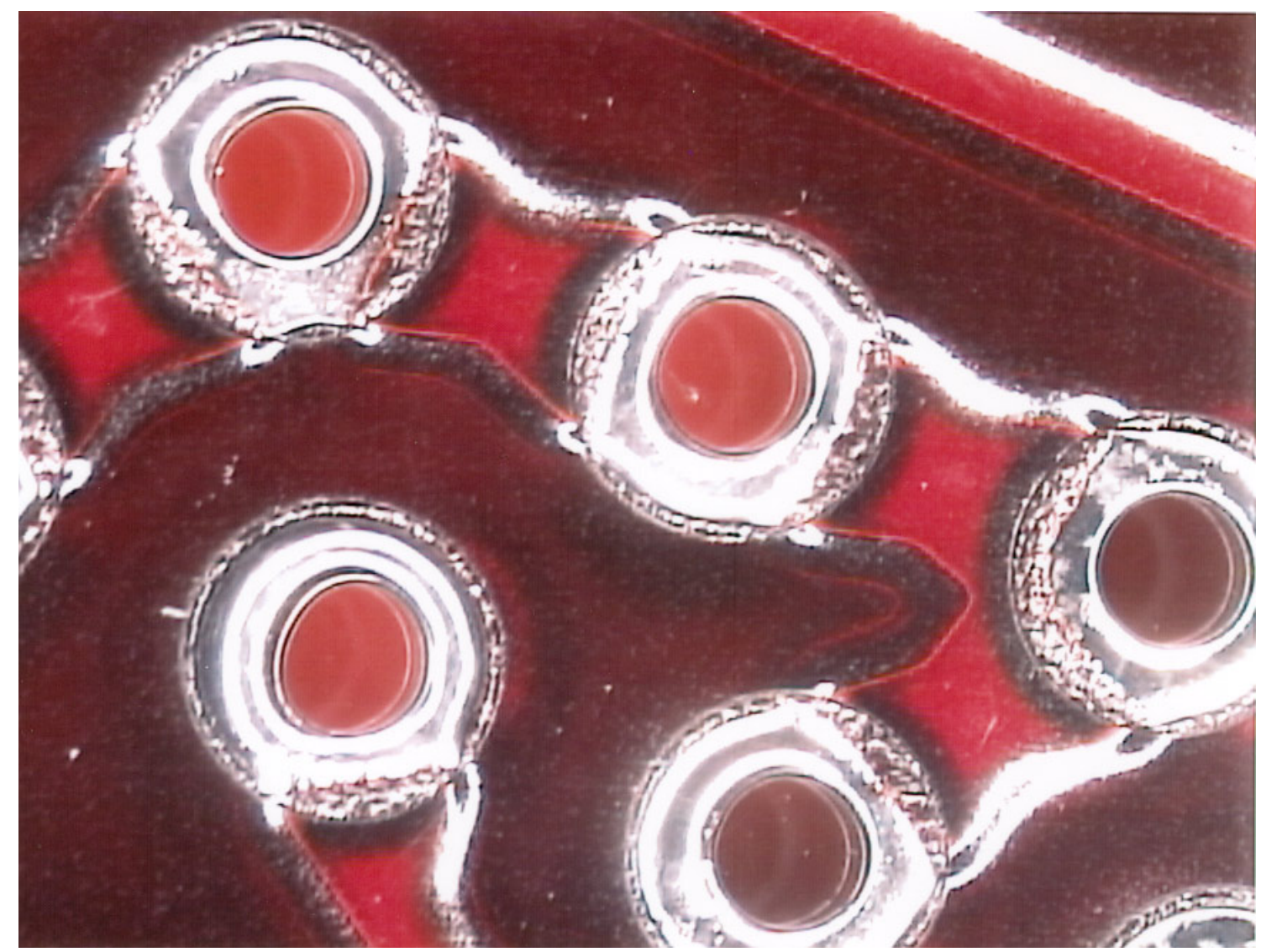

Figure 24. Annular Rings with Dark Halos 


\section{Statistical Analysis of Soft Press Pad Data}

The data from the fractional factorial design was analyzed using Minitab for $\mathrm{X}$ and $\mathrm{Y}$ axis stretch, delamination/solder wicking and the dark 'halo' around the annular ring. The results are listed below.

\section{Fractional Factorial Design}

$\begin{array}{lrlrlr}\text { Factors: } & 5 & \text { Base Design: } & 5,16 & \text { Resolution: } & \text { V } \\ \text { Runs: } & 33 & \text { Replicates: } & 2 & \text { Fraction: } & 1 / 2 \\ \text { Blocks: } & 1 & \text { Center pts (total): } & 1 & & \end{array}$

\section{Factorial Fit: X Axis Stretch}

Any P value less than .100 is significant. In this case although several factors appear statistically significant, as with previous experiments, the distribution is so far below that required for processing no variables are practically significant.

\begin{tabular}{|c|c|c|c|c|c|}
\hline Term & Effect & Coef & SE Coef & $\mathrm{T}$ & \\
\hline Constant & & 89.97 & 5.286 & 17.02 & $\odot .0 \odot \odot$ \\
\hline Lam Press & -26.37 & $-13 \cdot 18$ & 5.286 & -2.49 & $\odot .024$ \\
\hline Lam Temp & -9.53 & -4.76 & 5.286 & -0.90 & $\odot .381$ \\
\hline Lam Time & -11.01 & -5.50 & 5.286 & -1.04 & 0.313 \\
\hline Bake Time & $16 . \odot$ & $8.0 \odot$ & 5.286 & 1.51 & $\odot .150$ \\
\hline Bake Temp & & & .286 & $\odot .54$ & $\odot .598$ \\
\hline Lam Press*Lam Temp & -9.96 & -4.98 & 5.286 & -0.94 & 0.360 \\
\hline Lam Press*Lam Time & -15.11 & -7.56 & 5.286 & -1.43 & $\odot .172$ \\
\hline Lam Press*Bake Time & -8.77 & -4.38 & 5.286 & -0.83 & $\odot .419$ \\
\hline Lam Press*Bake Temp & 14.10 & 7.05 & 5.286 & 1.33 & $\odot .201$ \\
\hline Lam Temp*Lam Time & -3.24 & -1.62 & 5.286 & -0.31 & $\odot .763$ \\
\hline Lam Temp*Bake Time & 19.58 & 9.79 & 5.286 & 1.85 & $\odot .082$ \\
\hline Lam Temp*Bake Temp & -20.49 & -10.25 & 5.286 & -1.94 & $\odot .070$ \\
\hline Lam Time*Bake Time & -19.07 & -9.54 & 5.286 & -1.80 & $\odot .090$ \\
\hline Lam Time*Bake Temp & -20.71 & -10.36 & 5.286 & -1.96 & $\odot .068$ \\
\hline Bake Time*Bake Temp & 5.65 & 2.83 & 5.286 & 0.53 & 0.600 \\
\hline
\end{tabular}




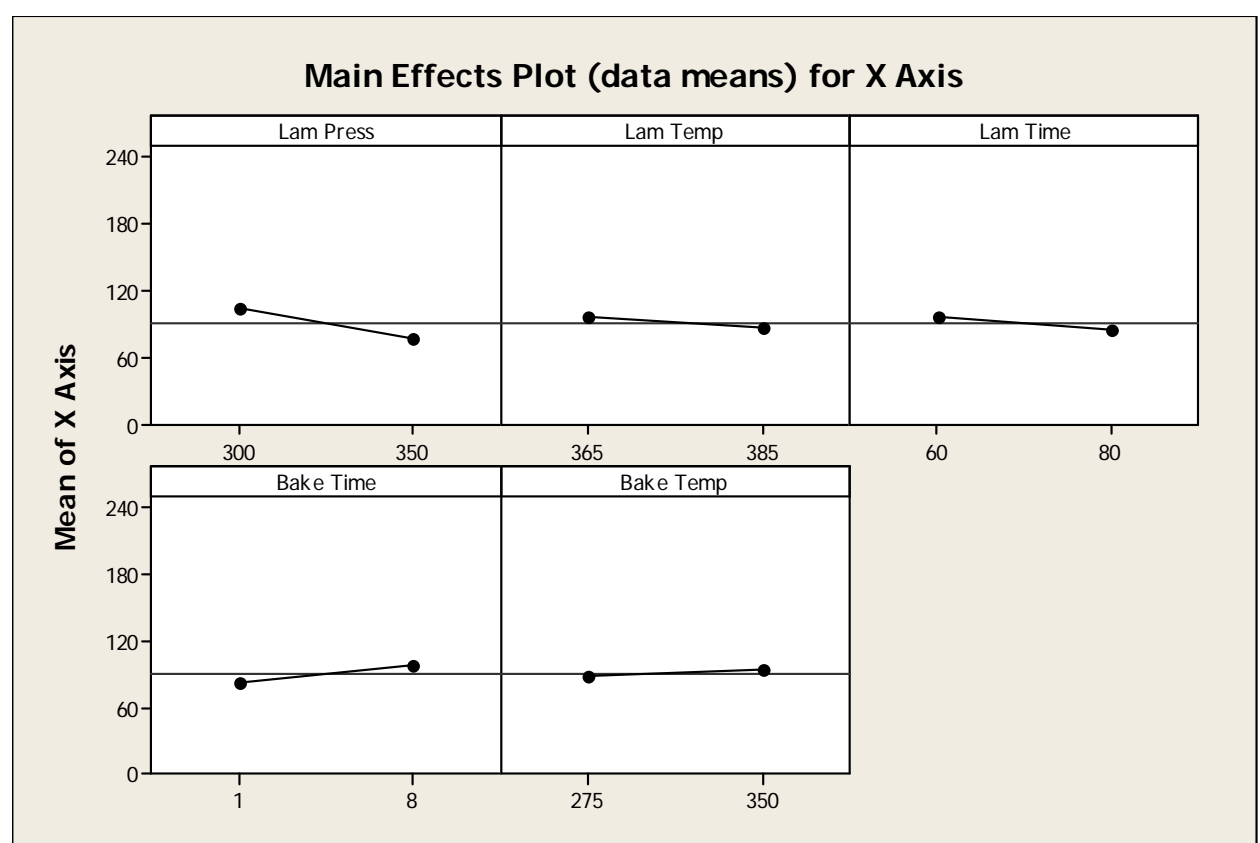

Figure 25. Main Effects Plot for X Axis

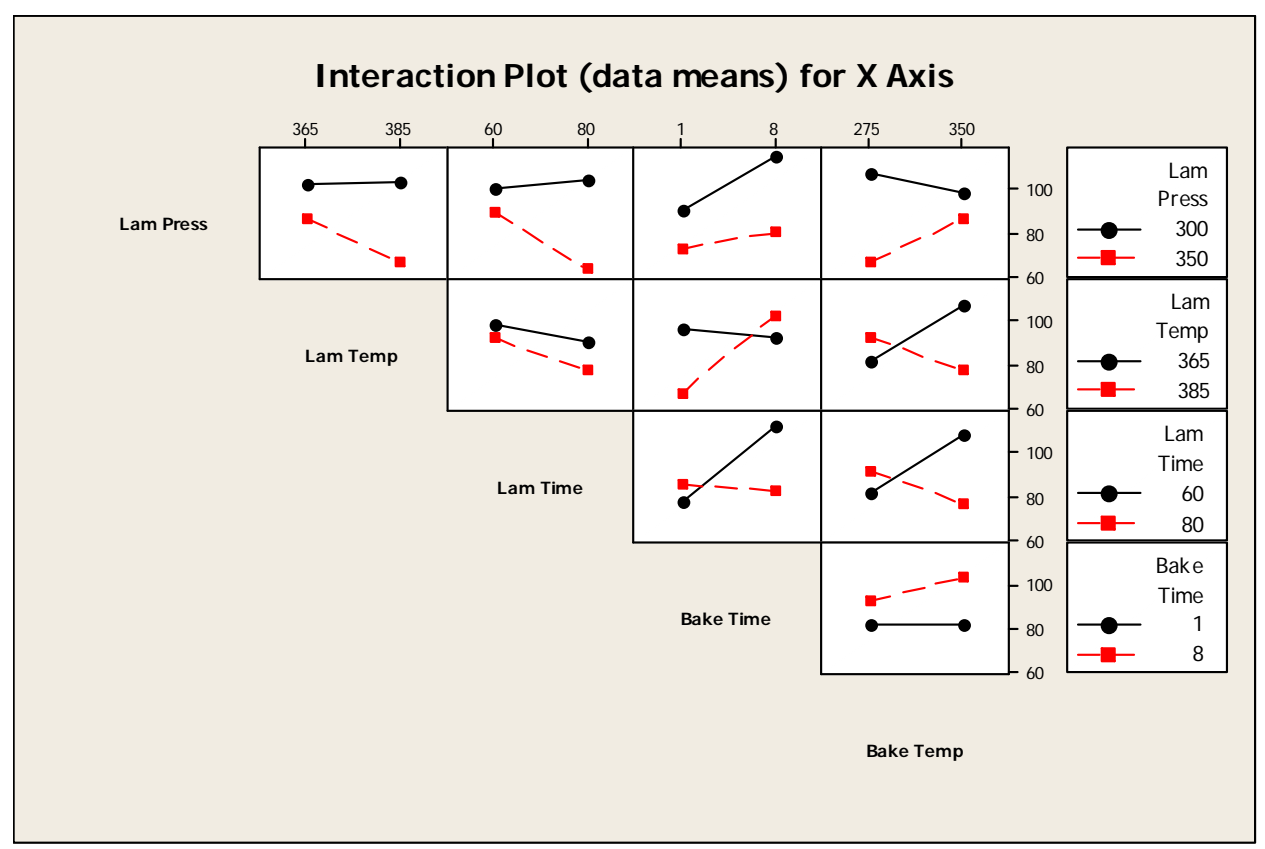

Figure 26. Interaction Plot for X Axis 
Factorial Fit: Y Axis Stretch

No variables were statistically or practically significant for $\mathrm{Y}$ axis stretch.

Estimated Effects and Coefficients for $Y$ Axis (coded units)

$\begin{array}{lrrrrr}\text { Term } & \text { Effect } & \text { Coef } & \text { SE Coef } & \text { T } & \text { P } \\ \text { Constant } & & 84.334 & 6.879 & 12.26 & 0.00 \odot \\ \text { Lam Press } & -8.089 & -4.045 & 6.879 & -0.59 & 0.565 \\ \text { Lam Temp } & -7.316 & -3.658 & 6.879 & -0.53 & 0.602 \\ \text { Lam Time } & -10.871 & -5.435 & 6.879 & -0.79 & 0.441 \\ \text { Bake Time } & 12.415 & 6.207 & 6.879 & 0.90 & 0.380 \\ \text { Bake Temp } & -0.834 & -0.417 & 6.879 & -0.06 & 0.952 \\ \text { Lam Press*Lam Temp } & 7.842 & 3.921 & 6.879 & 0.57 & 0.577 \\ \text { Lam Press*Lam Time } & -12.031 & -6.015 & 6.879 & -0.87 & 0.395 \\ \text { Lam Press*Bake Time } & -10.170 & -5.085 & 6.879 & -0.74 & 0.470 \\ \text { Lam Press*Bake Temp } & -11.745 & -5.873 & 6.879 & -0.85 & 0.406 \\ \text { Lam Temp*Lam Time } & 14.839 & 7.420 & 6.879 & 1.08 & 0.297 \\ \text { Lam Temp*Bake Time } & -11.765 & -5.882 & 6.879 & -0.86 & 0.405 \\ \text { Lam Temp*Bake Temp } & -3.043 & -1.522 & 6.879 & -0.22 & 0.828 \\ \text { Lam Time*Bake Time } & 14.708 & 7.354 & 6.879 & 1.07 & 0.301 \\ \text { Lam Time*Bake Temp } & -8.148 & -4.074 & 6.879 & -0.59 & 0.562 \\ \text { Bake Time*Bake Temp } & 1.338 & 0.669 & 6.879 & 0.10 & 0.924\end{array}$

$S=38.9115 \quad R-S q=33.01 \% \quad R-S q(\operatorname{adj})=0.00 \%$

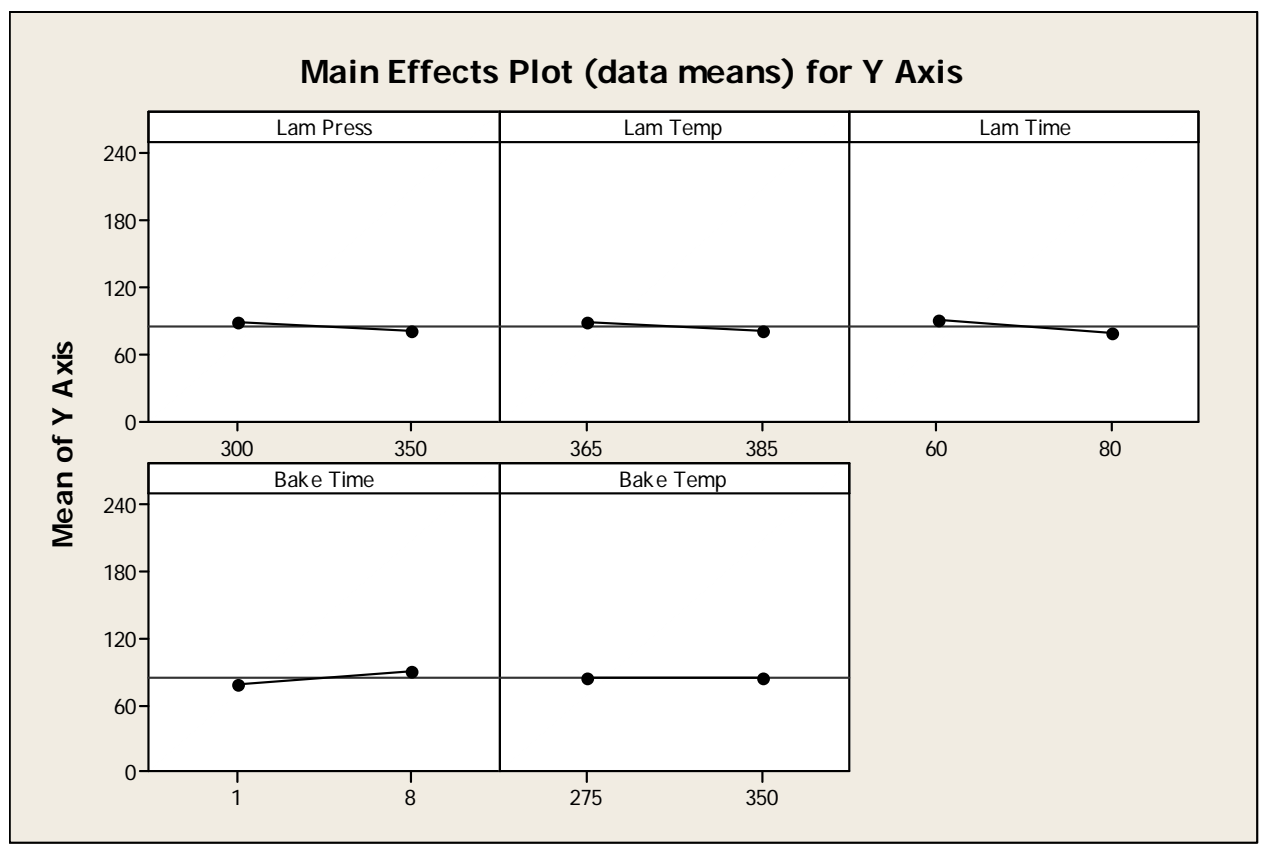

Figure 27. Main Effects Plot for Y Axis 


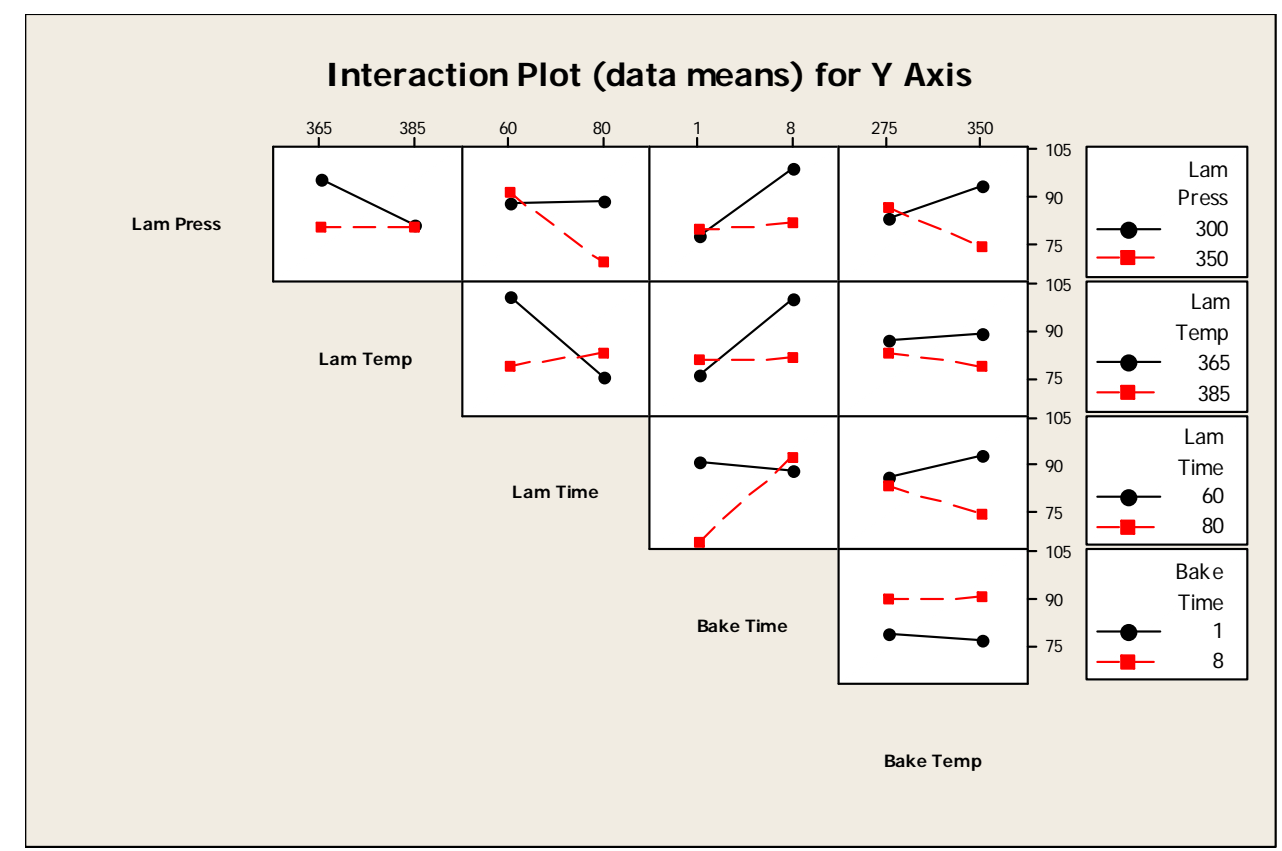

Figure 28. Interaction Plot for Y Axis

\section{Factorial Fit: Wicking}

All delamination/solder wicking variables were statistically significant. However, the Main Effects plot illustrates the effects of bake time and temperature were significantly greater then the other variables.

\begin{tabular}{|c|c|c|c|c|c|}
\hline Term & Effect & Coef & SE Coef & $\mathrm{T}$ & \\
\hline Constant & & 0.1366 & $\odot .003605$ & 37.90 & 0.000 \\
\hline Lam Press & 0.0277 & 0.0139 & $\odot .003605$ & 3.85 & 0.001 \\
\hline Lam Temp & -0.1035 & -0.0518 & $\odot .003605$ & -14.36 & $\odot .000$ \\
\hline Lam Time & -0.0223 & -0.0111 & $\odot .003605$ & -3.09 & 0.007 \\
\hline Bake Time & -0.2570 & -0.1285 & 0.003605 & -35.64 & 0.000 \\
\hline Bake Temp & -0.2733 & -0.1366 & 0.003605 & -37.90 & $\odot .000$ \\
\hline Lam Press*Lam Temp & -0.0200 & -0.0100 & 0.003605 & -2.77 & 0.014 \\
\hline Lam Press*Lam Time & -0.1005 & -0.0503 & 0.003605 & -13.94 & 0.000 \\
\hline Lam Press*Bake Time & -0.0307 & -0.0154 & $\odot .003605$ & -4.26 & 0.001 \\
\hline Lam Press*Bake Temp & -0.0277 & -0.0139 & $\odot .003605$ & -3.85 & $\odot . \odot \odot 1$ \\
\hline Lam Temp*Lam Time & 0.0307 & 0.0154 & $\odot .003605$ & 4.26 & $\odot .001$ \\
\hline Lam Temp*Bake Time & 0.1005 & 0.0502 & 0.003605 & 13.94 & 0.000 \\
\hline Lam Temp*Bake Temp & 0.1035 & 0.0517 & $\odot .003605$ & 14.36 & $\odot .00 \odot$ \\
\hline Lam Time*Bake Time & 0.0200 & 0.0100 & 0.003605 & 2.77 & 0.014 \\
\hline Lam Time*Bake Temp & 0.0222 & 0.0111 & 0.003605 & 3.09 & $0.0 \odot 7$ \\
\hline Bake Time*Bake Temp & 0.2570 & 0.1285 & $\odot .003605$ & 35.64 & $\odot .000$ \\
\hline
\end{tabular}

$S=0.0203930 \quad R-S q=99.67 \% \quad R-S q(\operatorname{adj})=99.37 \%$ 


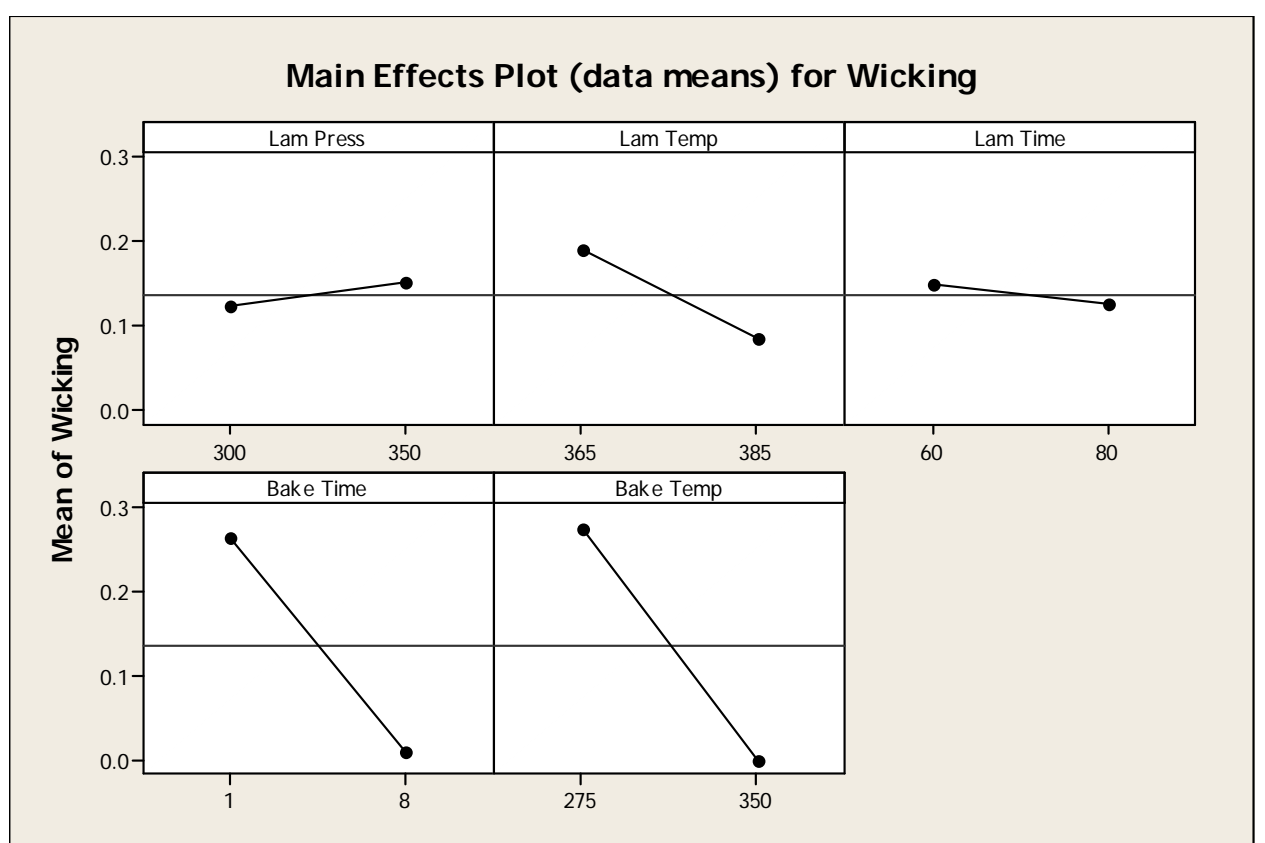

Figure 29. Main Effects Plot for Solder Wicking

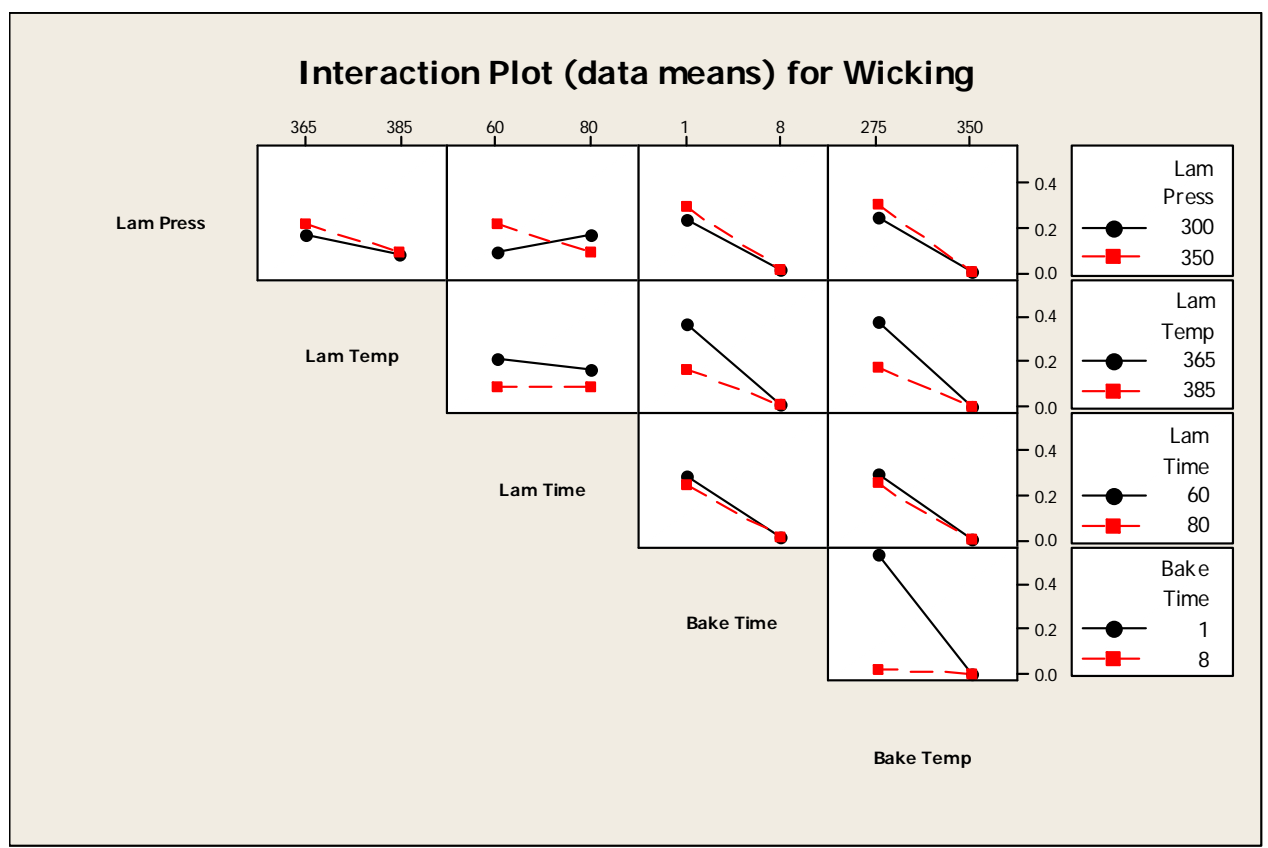

Figure 30. Interaction Plot for Solder Wicking 


\section{Factorial Fit: Dark Halos}

A dark 'halo' was observed under the coverlay around the annular rings during evaluation of the sample panels. Data analysis confirmed the dark 'halo' only occurred on sample panels baked at $350^{\circ} \mathrm{F}$. The dark 'halos' are thought to be oxidation and are not necessarily a defect, but could be considered foreign material.

\begin{tabular}{|c|c|c|c|c|c|}
\hline Term & Effect & Coef & SE Coef & $\mathrm{T}$ & $\mathrm{P}$ \\
\hline Constant & & 0.0625 & $\odot .06250$ & 1.00 & 0.332 \\
\hline Lam Press & 0.1250 & 0.0625 & $\odot .06250$ & 1.00 & 0.332 \\
\hline Lam Temp & -0.1250 & -0.0625 & 0.06250 & $-1.0 \odot$ & 0.332 \\
\hline Lam Time & -0.1250 & -0.0625 & 0.06250 & -1.00 & 0.332 \\
\hline Bake Time & 0.1250 & 0.0625 & 0.06250 & 1.00 & 0.332 \\
\hline Bake Temp & -1.8750 & -0.9375 & $\odot .06250$ & -15.00 & $\odot .0 \odot \odot$ \\
\hline Lam Press*Lam Temp & $-\odot .125 \odot$ & -0.0625 & $\odot .06250$ & $-1.0 \odot$ & 0.332 \\
\hline Lam Press*Lam Time & -0.1250 & -0.0625 & $\odot . \odot 625 \odot$ & $-1.0 \odot$ & 0.332 \\
\hline Lam Press*Bake Time & 0.1250 & 0.0625 & 0.06250 & 1.00 & 0.332 \\
\hline Lam Press*Bake Temp & 0.1250 & 0.0625 & $\odot .06250$ & 1.00 & 0.332 \\
\hline Lam Temp*Lam Time & 0.1250 & $\odot .0625$ & $\odot .06250$ & $1.0 \odot$ & $\odot .332$ \\
\hline Lam Temp*Bake Time & -0.1250 & -0.0625 & 0.06250 & -1.00 & 0.332 \\
\hline Lam Temp*Bake Temp & -0.1250 & -0.0625 & $\odot .06250$ & $-1.0 \odot$ & 0.332 \\
\hline Lam Time*Bake Time & -0.1250 & -0.0625 & $\odot .06250$ & $-1.0 \odot$ & $\odot .332$ \\
\hline Lam Time*Bake Temp & -0.1250 & -0.0625 & 0.06250 & -1.00 & 0.332 \\
\hline Bake Time* Bake Temp & 0.1250 & 0.0625 & $\odot .06250$ & 1.00 & 0.332 \\
\hline
\end{tabular}

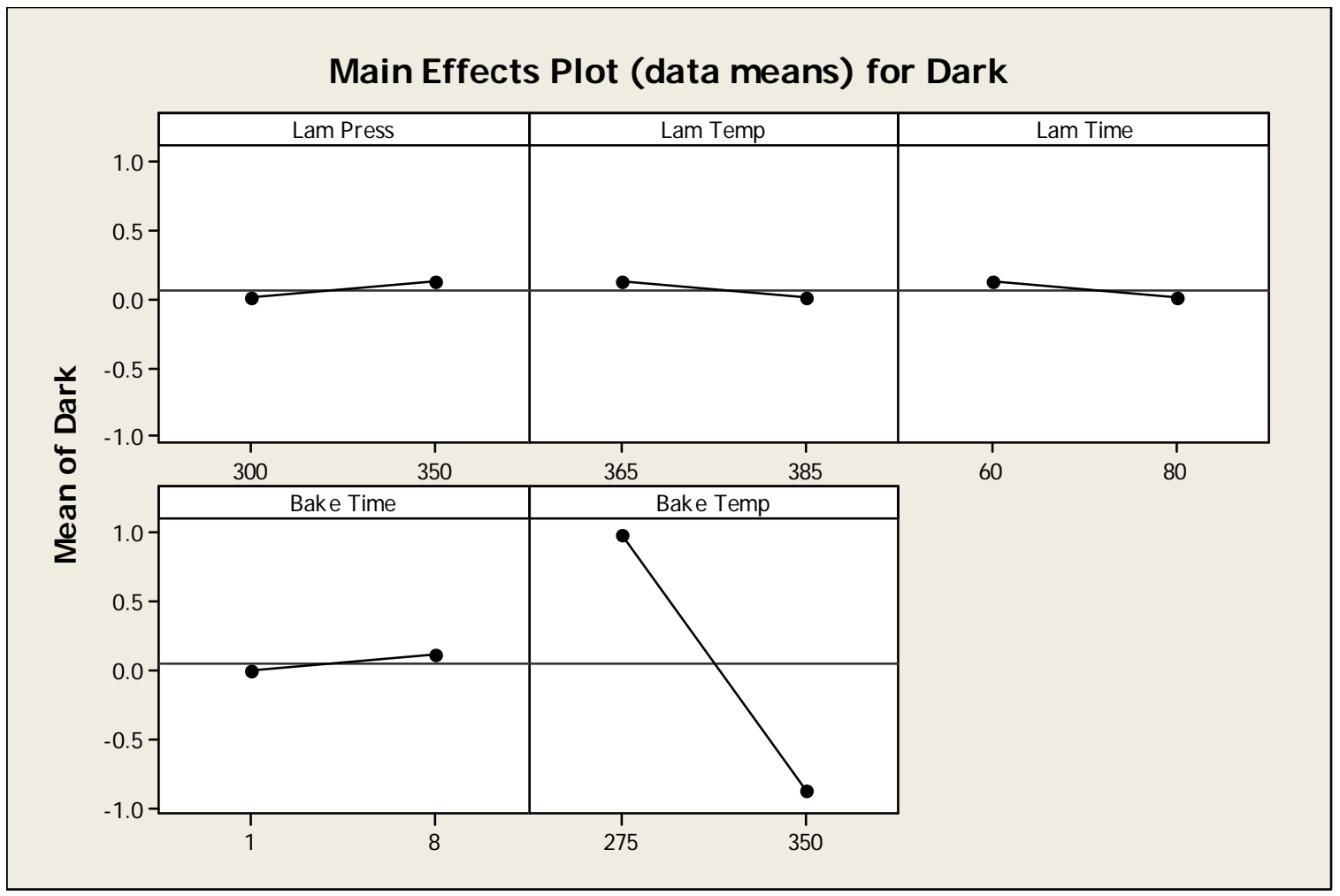

Figure 31. Main Effects Plot for Dark Halos 


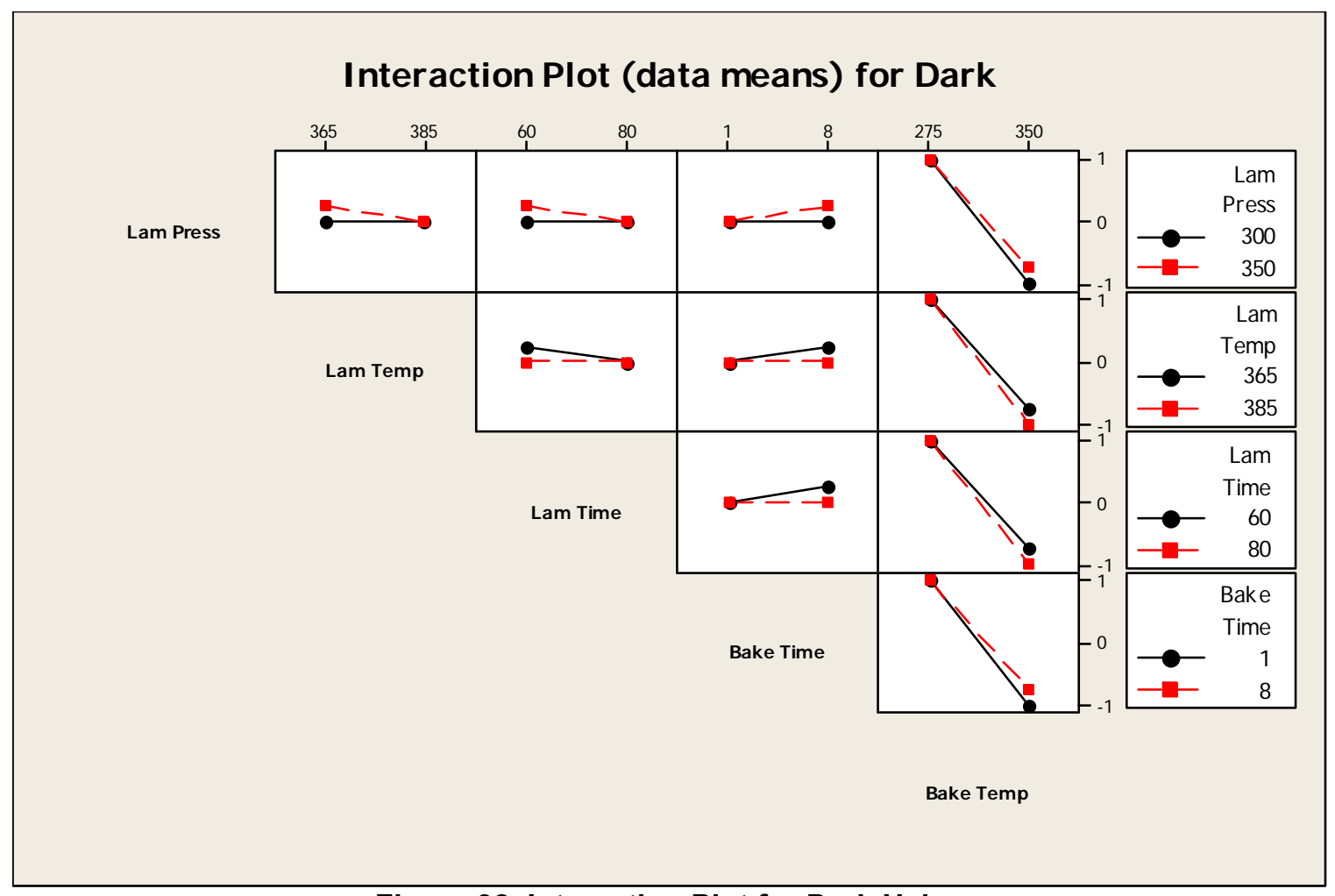

Figure 32. Interaction Plot for Dark Halos 


\begin{tabular}{|c|c|c|c|c|c|c|c|c|c|c|c|}
\hline StdOrder & RunOrder & CenterPt & Blocks & Lam Press & Lam Temp & Lam Time & Bake Time & Bake Temp & $X$ Axis & Y Axis & Wicking \\
\hline 7 & 1 & 1 & 1 & 300 & 385 & 80 & 1 & 350 & 57.987 & 71.608 & 0 \\
\hline 8 & 2 & 1 & 1 & 350 & 385 & 80 & 1 & 275 & 65.946 & 38.329 & 0.325 \\
\hline 19 & 3 & 1 & 1 & 300 & 385 & 60 & 1 & 275 & 73.434 & 47.307 & 0.34 \\
\hline 28 & 4 & 1 & 1 & 350 & 385 & 60 & 8 & 275 & 132.105 & 39.74 & 0.015 \\
\hline 29 & 5 & 1 & 1 & 300 & 365 & 80 & 8 & 350 & 88.158 & 125.376 & 0 \\
\hline 20 & 6 & 1 & 1 & 350 & 385 & 60 & 1 & 350 & 41.538 & 115.61 & 0 \\
\hline 14 & 7 & 1 & 1 & 350 & 365 & 80 & 8 & 275 & 51.274 & 102.554 & 0.003 \\
\hline 6 & 8 & 1 & 1 & 350 & 365 & 80 & 1 & 350 & 110.304 & 29.529 & 0 \\
\hline 9 & 9 & 1 & 1 & 300 & 365 & 60 & 8 & 275 & 102.302 & 137.433 & 0.01 \\
\hline 1 & 10 & 1 & 1 & 300 & 365 & 60 & 1 & 350 & 114.811 & 152.622 & 0 \\
\hline 16 & 11 & 1 & 1 & 350 & 385 & 80 & 8 & 350 & 43.387 & 83.097 & 0 \\
\hline 21 & 12 & 1 & 1 & 300 & 365 & 80 & 1 & 275 & 168.059 & 58.007 & 0.667 \\
\hline 15 & 13 & 1 & 1 & 300 & 385 & 80 & 8 & 275 & 133.041 & 112.415 & 0.008 \\
\hline 26 & 14 & 1 & 1 & 350 & 365 & 60 & 8 & 350 & 93.077 & 80.051 & 0 \\
\hline 2 & 15 & 1 & 1 & 350 & 365 & 60 & 1 & 275 & 82.717 & 140.6 & 0.81 \\
\hline 11 & 16 & 1 & 1 & 300 & 385 & 60 & 8 & 350 & 129.892 & 110.961 & 0 \\
\hline 23 & 17 & 1 & 1 & 300 & 385 & 80 & 1 & 350 & 69.065 & 78.844 & 0 \\
\hline 24 & 18 & 1 & 1 & 350 & 385 & 80 & 1 & 275 & 58.086 & 134.634 & 0.36 \\
\hline 3 & 19 & 1 & 1 & 300 & 385 & 60 & 1 & 275 & 82.851 & 87.503 & 0.28 \\
\hline 12 & 20 & 1 & 1 & 350 & 385 & 60 & 8 & 275 & 51.561 & 111.334 & 0.01 \\
\hline 13 & 21 & 1 & 1 & 300 & 365 & 80 & 8 & 350 & 108.193 & 109.06 & 0 \\
\hline 4 & 22 & 1 & 1 & 350 & 385 & 60 & 1 & 350 & 90.365 & 68.973 & 0 \\
\hline 30 & 23 & 1 & 1 & 350 & 365 & 80 & 8 & 275 & 42.264 & 64.823 & 0.025 \\
\hline 22 & 24 & 1 & 1 & 350 & 365 & 80 & 1 & 350 & 85.216 & 45.045 & 0 \\
\hline 25 & 25 & 1 & 1 & 300 & 365 & 60 & 8 & 275 & 82.027 & 64.364 & 0.039 \\
\hline 17 & 26 & 1 & 1 & 300 & 365 & 60 & 1 & 350 & 86.585 & 54.094 & 0 \\
\hline 32 & 27 & 1 & 1 & 350 & 385 & 80 & 8 & 350 & 53.306 & 52.698 & 0 \\
\hline 5 & 28 & 1 & 1 & 300 & 365 & 80 & 1 & 275 & 73.328 & 66.703 & 0.6 \\
\hline 31 & 29 & 1 & 1 & 300 & 385 & 80 & 8 & 275 & 143.796 & 89.654 & 0.02 \\
\hline 10 & 30 & 1 & 1 & 350 & 365 & 60 & 8 & 350 & 176.231 & 116.993 & 0 \\
\hline 18 & 31 & 1 & 1 & 350 & 365 & 60 & 1 & 275 & 51.159 & 60.618 & 0.86 \\
\hline 27 & 32 & 1 & 1 & 300 & 385 & 60 & 8 & 350 & 136.881 & 48.105 & 0 \\
\hline
\end{tabular}




\section{Conclusions}

A pre-HASL bake temperature higher than the current $275^{\circ} \mathrm{F}$ is required to potentially eliminate delamination/solder wicking when using the soft press pad stackup. A followon evaluation will be conducted to determine a temperature and time that will eliminate the wicking without the 'halo' effect.

As with previous evaluations, the amount of stretch in the $\mathrm{X}$ and $\mathrm{Y}$ axis is negligible when using the soft press pad stackup.

See Appendix D for results of environmental testing performed by Trace Laboratories.

\section{Sample (One Ounce Copper Laminate)}

This experiment was performed to determine if flat flex cables containing one ounce copper laminate would have similar results as the two ounce copper laminates and to determine lamination and pre-HASL bake parameters that reduced or eliminated delamination/solder wicking. This was an effort to accomplish two goals with one experiment without diluting the experiment. But there was some concern the experiment contained too many variables since the results did not confirm earlier results from the two ounce laminate experiment.

A twelve-by-twelve inch, one ounce copper, double-sided laminate sample was generated which contained the circuit patterns of five cables. The coverlay laminated to both sides of the laminate samples was two mil polyimide/one mil pyralux adhesive. Nine holes with a diameter of .060 inch were strategically located on the sample panel for CMM hole location measurements.

\section{Experiment}

A fractional factorial experiment with two independent variables was developed. Six factors were selected for each variable as shown in the table below.

\begin{tabular}{|c|c|c|}
\hline Variable (Pre HASL Bake) & Level & Level \\
\hline Bake Temperature & $300 \mathrm{~F}$ & $325 \mathrm{~F}$ \\
\hline Bake Time & $1,2 \& 4$ hours & $1,2 \& 4$ hours \\
\hline
\end{tabular}

Six sample panels were processed for each variable and factor combination resulting in thirty-six panels total. All were laminated in the TMP lamination press over a period of three days using a pressure of $350 \mathrm{psi}$, a temperature of $385^{\circ} \mathrm{F}$ and a time of 60 minutes. Each lamination cycle's actual temperature, pressure, and soak time were recorded at one minute intervals. After lamination each panel's nine holes were measured for location by a department's CMM and recorded. The pre-HASL bake was performed per the above variable/factor table. Below is a process map of lamination through HASL.

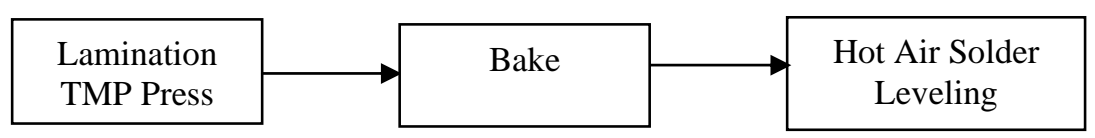


Measuring the depth of delamination/solder wicking on each land of all five cables per panel was determined to be an acceptable method of quantifying adhesion quality. The greater the solder wicking the more likely there is inadequate adhesion. The total number of lands per panel was seventy.

\section{Adhesion Quality Analysis}

The table on the next page summarizes each panels ranking as a sum of each parts ranking. The fill color highlights each pre-HASL bake temperature and time. When comparing solder wicking rank of each colored group highlighted it is slightly evident a longer pre-HASL bake would reduce delamination/solder wicking when using a soft press pad stackup. See Appendix C for each individual panel ranking (magnitude of delamination/solder wicking).

\section{Conclusions}

When performing this experiment with one ounce copper laminate cables, there was an increase in delamination/solder wicking with all variable combinations. Data analysis indicates longer HASL pre-bake times will produce less delamination/solder wicking. This data implies different processing for two and one ounce copper laminates.

As with previous evaluations the amount of stretch in the $\mathrm{X}$ and $\mathrm{Y}$ axis is negligible when using the soft press pad stackup.

See Appendix D for results of environmental testing performed by Trace Laboratories.

\section{Future Work}

Future work will emphasize activities related to determining optimized lamination, preHASL bake and pre-HASL clean parameters:

1) Determining proper bake parameters when using the soft press pad stackup.

2) Evaluating the elimination of the bake process when using the hard press pad stackup.

3) Determining the interaction between lower lamination pressure and pre-HASL bake process when using the hard press pad stackup.

Future work will concentrate on determining process repeatability and part acceptability when changing process parameters. 


\begin{tabular}{|c|c|c|c|c|c|c|c|c|c|c|c|c|c|c|c|c|c|}
\hline \multicolumn{18}{|l|}{ Soft Press Pad Sy } \\
\hline Panel \# & A & B & $\begin{array}{l}\text { Total per } \\
\text { Part }\end{array}$ & A & B & $\begin{array}{l}\text { Total per } \\
\text { Part }\end{array}$ & A & B & $\begin{array}{c}\text { Total per } \\
\text { Part }\end{array}$ & A & i & $\begin{array}{c}\text { Total per } \\
\text { Part }\end{array}$ & A & B & $\begin{array}{c}\text { Total per } \\
\text { Part }\end{array}$ & $\begin{array}{c}\text { Total per } \\
\text { Panel }\end{array}$ & DoE Parameters \\
\hline 9 & 0.000 & 0.000 & 0.000 & 0.000 & 0.000 & 0.000 & 0.015 & 0.000 & 0.015 & 0.018 & 0.018 & 0.036 & 0.013 & 0.014 & 0.027 & 0.078 & $300 \mathrm{~F} 4$ hours \\
\hline 12 & 0.004 & 0.000 & 0.004 & 0.000 & 0.000 & 0.000 & 0.019 & 0.010 & 0.029 & 0.013 & 0.018 & 0.031 & 0.016 & 0.018 & 0.034 & 0.098 & $325 \mathrm{~F} 4$ hours \\
\hline 2 & 0.000 & 0.000 & 0.000 & 0.000 & 0.000 & 0.000 & 0.027 & 0.009 & 0.036 & 0.028 & 0.006 & 0.034 & 0.017 & 0.014 & 0.031 & 0.101 & $300 \mathrm{~F} 4$ hours \\
\hline 4 & 0.004 & 0.000 & 0.004 & 0.005 & 0.000 & 0.005 & 0.028 & 0.006 & 0.034 & 0.035 & 0.007 & 0.042 & 0.015 & 0.011 & 0.026 & 0.111 & $325 \mathrm{~F} 4$ hours \\
\hline 8 & 0.000 & 0.004 & 0.004 & 0.000 & 0.004 & 0.004 & 0.025 & 0.006 & 0.031 & 0.050 & 0.007 & 0.057 & 0.020 & 0.004 & 0.024 & 0.120 & $325 \mathrm{~F} 4$ hours \\
\hline 17 & 0.000 & 0.000 & 0.000 & 0.000 & 0.000 & 0.000 & 0.020 & 0.018 & 0.038 & 0.021 & 0.034 & 0.055 & 0.040 & 0.007 & 0.047 & 0.140 & $325 \mathrm{~F} 2$ hours \\
\hline 10 & 0.000 & 0.000 & 0.000 & 0.005 & 0.002 & 0.007 & 0.025 & 0.032 & 0.057 & 0.026 & 0.031 & 0.057 & 0.022 & 0.011 & 0.033 & 0.154 & $325 \mathrm{~F} 2$ hours \\
\hline 14 & 0.000 & 0.000 & 0.000 & 0.007 & 0.000 & 0.007 & 0.035 & 0.014 & 0.049 & 0.036 & 0.024 & 0.060 & 0.030 & 0.010 & 0.040 & 0.156 & $300 \mathrm{~F} 4$ hours \\
\hline 21 & 0.000 & 0.000 & 0.000 & 0.002 & 0.000 & 0.002 & 0.019 & 0.038 & 0.057 & 0.053 & 0.038 & 0.091 & 0.031 & 0.015 & 0.046 & 0.196 & $300 \mathrm{~F} 2$ hours \\
\hline 6 & 0.000 & 0.005 & 0.005 & 0.000 & 0.000 & 0.000 & 0.033 & 0.024 & 0.057 & 0.066 & 0.023 & 0.089 & 0.027 & 0.019 & 0.046 & 0.197 & $325 \mathrm{~F} 2$ hours \\
\hline 27 & 0.008 & 0.008 & 0.016 & 0.006 & 0.000 & 0.006 & 0.035 & 0.028 & 0.063 & 0.056 & 0.028 & 0.084 & 0.024 & 0.016 & 0.040 & 0.209 & $300 \mathrm{~F} 4$ hours \\
\hline 15 & 0.000 & 0.000 & 0.000 & 0.017 & 0.000 & 0.017 & 0.051 & 0.010 & 0.061 & 0.064 & 0.013 & 0.077 & 0.034 & 0.025 & 0.059 & 0.214 & $325 \mathrm{~F} 1$ hour \\
\hline 18 & 0.000 & 0.000 & 0.000 & 0.024 & 0.000 & 0.024 & 0.030 & 0.030 & 0.060 & 0.050 & 0.035 & 0.085 & 0.032 & 0.015 & 0.047 & 0.216 & $300 \mathrm{~F} 2$ hours \\
\hline 11 & 0.000 & 0.009 & 0.009 & 0.004 & 0.000 & 0.004 & 0.032 & 0.035 & 0.067 & 0.042 & 0.041 & 0.083 & 0.029 & 0.027 & 0.056 & 0.219 & $300 \mathrm{~F} 2$ hours \\
\hline 22 & 0.005 & 0.005 & 0.010 & 0.019 & 0.000 & 0.019 & 0.057 & 0.021 & 0.078 & 0.064 & 0.011 & 0.075 & 0.036 & 0.003 & 0.039 & 0.221 & $325 \mathrm{~F} 4$ hours \\
\hline 16 & 0.000 & 0.000 & 0.000 & 0.014 & 0.000 & 0.014 & 0.037 & 0.027 & 0.064 & 0.055 & 0.039 & 0.094 & 0.044 & 0.013 & 0.057 & 0.229 & $325 \mathrm{~F} 1$ hour \\
\hline 26 & 0.005 & 0.010 & 0.015 & 0.023 & 0.000 & 0.023 & 0.033 & 0.032 & 0.065 & 0.063 & 0.028 & 0.091 & 0.024 & 0.012 & 0.036 & 0.230 & $325 \mathrm{~F} 4$ hours \\
\hline 30 & 0.005 & 0.005 & 0.010 & 0.019 & 0.000 & 0.019 & 0.039 & 0.033 & 0.072 & 0.048 & 0.047 & 0.095 & 0.027 & 0.013 & 0.040 & 0.236 & $325 \mathrm{~F} 4$ hours \\
\hline 28 & 0.000 & 0.009 & 0.009 & 0.002 & 0.004 & 0.006 & 0.032 & 0.038 & 0.070 & 0.061 & 0.050 & 0.111 & 0.022 & 0.022 & 0.044 & 0.240 & $325 \mathrm{~F} 2$ hours \\
\hline 20 & 0.007 & 0.015 & 0.022 & 0.018 & 0.005 & 0.023 & 0.036 & 0.029 & 0.065 & 0.060 & 0.034 & 0.094 & 0.026 & 0.014 & 0.040 & 0.244 & $300 \mathrm{~F} 4$ hours \\
\hline 32 & 0.010 & 0.008 & 0.018 & 0.014 & 0.016 & 0.030 & 0.012 & 0.032 & 0.044 & 0.069 & 0.048 & 0.117 & 0.025 & 0.018 & 0.043 & 0.252 & $300 \mathrm{~F} 4$ hours \\
\hline 35 & 0.009 & 0.008 & 0.017 & 0.000 & 0.004 & 0.004 & 0.032 & 0.045 & 0.077 & 0.062 & 0.050 & 0.112 & 0.026 & 0.019 & 0.045 & 0.255 & $325 \mathrm{~F} 2$ hours \\
\hline 24 & 0.000 & 0.008 & 0.008 & 0.010 & 0.002 & 0.012 & 0.033 & 0.039 & 0.072 & 0.069 & 0.047 & 0.116 & 0.029 & 0.019 & 0.048 & 0.256 & $325 \mathrm{~F} 2$ hours \\
\hline 3 & 0.000 & 0.000 & 0.000 & 0.006 & 0.000 & 0.006 & 0.045 & 0.034 & 0.079 & 0.072 & 0.034 & 0.106 & 0.079 & 0.022 & 0.101 & 0.292 & $300 \mathrm{~F} 2$ hours \\
\hline 36 & 0.011 & 0.005 & 0.016 & 0.008 & 0.000 & 0.008 & 0.064 & 0.041 & 0.105 & 0.067 & 0.067 & 0.134 & 0.047 & 0.029 & 0.076 & 0.339 & $300 \mathrm{~F} 2$ hours \\
\hline 34 & 0.006 & 0.017 & 0.023 & 0.020 & 0.012 & 0.032 & 0.041 & 0.050 & 0.091 & 0.075 & 0.062 & 0.137 & 0.039 & 0.020 & 0.059 & 0.342 & $325 \mathrm{~F} 1$ hour \\
\hline 29 & 0.009 & 0.014 & 0.023 & 0.020 & 0.016 & 0.036 & 0.058 & 0.058 & 0.116 & 0.070 & 0.067 & 0.137 & 0.042 & 0.040 & 0.082 & 0.394 & $300 \mathrm{~F} 2$ hours \\
\hline 13 & 0.018 & 0.012 & 0.030 & 0.026 & 0.000 & 0.026 & 0.048 & 0.034 & 0.082 & 0.102 & 0.079 & 0.181 & 0.071 & 0.055 & 0.126 & 0.445 & $300 \mathrm{~F} 1$ hour \\
\hline 23 & 0.000 & 0.003 & 0.003 & 0.017 & 0.002 & 0.019 & 0.034 & 0.050 & 0.084 & 0.060 & 0.069 & 0.129 & 0.228 & 0.025 & 0.253 & 0.488 & $325 \mathrm{~F} 1$ hour \\
\hline 5 & 0.004 & 0.006 & 0.010 & 0.010 & 0.000 & 0.010 & 0.047 & 0.034 & 0.081 & 0.078 & 0.035 & 0.113 & 0.282 & 0.026 & 0.308 & 0.522 & $325 \mathrm{~F} 1$ hour \\
\hline 19 & 0.004 & 0.017 & 0.021 & 0.025 & 0.000 & 0.025 & 0.061 & 0.031 & 0.092 & 0.112 & 0.067 & 0.179 & 0.144 & 0.070 & 0.214 & 0.531 & $300 \mathrm{~F} 1$ hour \\
\hline 1 & 0.000 & 0.013 & 0.013 & 0.027 & 0.000 & 0.027 & 0.049 & 0.059 & 0.108 & 0.106 & 0.040 & 0.146 & 0.212 & 0.044 & 0.256 & 0.550 & 300F 1 hour \\
\hline 7 & 0.015 & 0.015 & 0.030 & 0.010 & 0.022 & 0.032 & 0.051 & 0.077 & 0.128 & 0.122 & 0.092 & 0.214 & 0.120 & 0.047 & 0.167 & 0.571 & $300 \mathrm{~F} 1$ hour \\
\hline 31 & 0.019 & 0.013 & 0.032 & 0.032 & 0.019 & 0.051 & 0.078 & 0.066 & 0.144 & 0.118 & 0.079 & 0.197 & 0.114 & 0.083 & 0.197 & 0.621 & $300 \mathrm{~F} 1$ hour \\
\hline 25 & 0.010 & 0.020 & 0.030 & 0.022 & 0.000 & 0.022 & 0.057 & 0.029 & 0.086 & 0.123 & 0.089 & 0.212 & 0.280 & 0.069 & 0.349 & 0.699 & $300 \mathrm{~F} 1$ hour \\
\hline 33 & 0.012 & 0.022 & 0.034 & 0.031 & 0.000 & 0.031 & 0.069 & 0.050 & 0.119 & 0.124 & 0.098 & 0.222 & 0.478 & 0.808 & 1.286 & 1.692 & 325F 1 hour \\
\hline Total & & & 0.416 & & & 0.551 & & & 2.576 & & & 3.893 & & & 4.422 & 11.858 & \\
\hline Hard Press Pad S & stem (1 & loz) & & & & & & & & & & & & & & & \\
\hline Panel \# & : & B & $\begin{array}{c}\text { Total per } \\
\text { Part }\end{array}$ & A & B & $\begin{array}{l}\text { Total per } \\
\text { Part }\end{array}$ & A & B & $\begin{array}{c}\text { Total per } \\
\text { Part }\end{array}$ & A & B & $\begin{array}{c}\text { Total per } \\
\text { Part }\end{array}$ & A & B & $\begin{array}{l}\text { Total per } \\
\text { Part }\end{array}$ & $\begin{array}{c}\text { Total per } \\
\text { Panel }\end{array}$ & DoE Parameters \\
\hline 1 & 0.000 & 0.000 & 0.000 & 0.000 & 0.000 & 0.000 & 0.002 & 0.002 & 0.004 & 0.002 & 0.000 & 0.002 & 0.007 & 0.000 & 0.007 & 0.013 & 190 ton \\
\hline 2 & 0.000 & 0.000 & 0.000 & 0.000 & 0.000 & 0.000 & 0.000 & 0.021 & 0.021 & 0.000 & 0.007 & 0.007 & 0.020 & 0.000 & 0.020 & 0.048 & 190 ton \\
\hline 3 & 0.000 & 0.000 & 0.000 & 0.000 & 0.000 & 0.000 & 0.003 & 0.000 & 0.003 & 0.000 & 0.000 & 0.000 & 0.010 & 0.000 & 0.010 & 0.013 & 190 ton \\
\hline 8 & 0.000 & 0.000 & 0.000 & 0.000 & 0.000 & 0.000 & 0.008 & 0.004 & 0.012 & 0.007 & 0.000 & 0.007 & 0.015 & 0.000 & 0.015 & 0.034 & 100 ton \\
\hline 9 & 0.000 & 0.000 & 0.000 & 0.004 & 0.000 & 0.004 & 0.002 & 0.011 & 0.013 & 0.006 & 0.000 & 0.006 & 0.011 & 0.000 & 0.011 & 0.034 & 100 ton \\
\hline
\end{tabular}

Table 3. Panel Ranking 


\section{Appendix A \\ Phase One Data}

\begin{tabular}{|c|c|c|c|c|c|c|c|c|c|c|c|c|c|c|}
\hline \multirow{3}{*}{$\begin{array}{c}\text { PNL } \\
\#\end{array}$} & \multirow{3}{*}{$\begin{array}{c}\text { Press } \\
\text { Pad }\end{array}$} & \multirow{3}{*}{$\begin{array}{l}\text { Pre- } \\
\text { Bake }\end{array}$} & \multicolumn{12}{|c|}{ As Measured on OGP } \\
\hline & & & \multicolumn{3}{|c|}{ Upper Left } & \multicolumn{3}{|c|}{ Upper Right } & \multicolumn{3}{|c|}{ Lower Right } & \multicolumn{3}{|c|}{ Lower Left } \\
\hline & & & Dia. & $\mathrm{x}$ & $\mathbf{Y}$ & Dia. & $\mathrm{X}$ & $\mathbf{Y}$ & Dia. & $\mathbf{x}$ & $\mathbf{Y}$ & Dia. & $\mathbf{x}$ & $\mathbf{Y}$ \\
\hline 1 & Hard & $\mathrm{N}$ & 0.09256 & 0.00000 & 0.00000 & 0.09336 & 6.76706 & -0.00017 & 0.09283 & 6.76696 & -4.99075 & 0.09280 & 0.00181 & -4.99437 \\
\hline 2 & Hard & $\mathrm{N}$ & 0.09281 & 0.00000 & 0.00000 & 0.09311 & 6.76421 & -0.00002 & 0.09298 & 6.76344 & -4.99171 & 0.09300 & -0.00121 & -4.99061 \\
\hline 3 & Soft & $\bar{N}$ & 0.09321 & 0.00000 & 0.00000 & 0.09305 & 6.76390 & 0.00003 & 0.09282 & 6.76428 & -4.98473 & 0.09273 & -0.00026 & -4.98455 \\
\hline 4 & Hard & $\mathrm{Y}$ & 0.09295 & 0.00000 & 0.00000 & 0.09259 & 6.76513 & -0.00002 & 0.09278 & 6.76499 & -4.99314 & 0.09243 & -0.00097 & -4.98930 \\
\hline 5 & Soft & $\mathrm{Y}$ & 0.09293 & 0.00000 & 0.00000 & 0.09314 & 6.76403 & -0.00006 & 0.09298 & 6.76394 & -4.98506 & 0.09298 & 0.00007 & -4.98414 \\
\hline 6 & Hard & $\mathrm{Y}$ & 0.09313 & 0.00000 & 0.00000 & 0.09300 & 6.76296 & 0.00006 & 0.09294 & 6.76395 & -4.99019 & 0.09265 & 0.00044 & -4.99422 \\
\hline 7 & Hard & $Y$ & 0.09315 & 0.00000 & 0.00000 & 0.09322 & 6.76280 & 0.00008 & 0.09284 & 6.76270 & -4.98924 & 0.09283 & 0.00000 & -4.98856 \\
\hline 8 & Soft & $\mathrm{Y}$ & 0.09291 & 0.00000 & 0.00000 & 0.09306 & 6.76365 & 0.00000 & 0.09308 & 6.76374 & -4.98425 & 0.09289 & -0.00012 & \\
\hline 9 & Hard & $\mathrm{N}$ & 0.09303 & 0.00000 & 0.00000 & 0.09327 & 6.76529 & -0.00001 & 0.09291 & 6.76153 & -4.99538 & 0.09273 & -0.00325 & \\
\hline 10 & Soft & $\mathrm{N}$ & 0.09283 & 0.00000 & 0.00000 & 0.09319 & 6.76388 & 0.00001 & 0.09252 & 6.76504 & -4.98510 & 0.09277 & 0.00092 & -4.98475 \\
\hline 11 & Soft & $\mathrm{N}$ & 0.09294 & 0.00000 & 0.00000 & 0.09309 & 6.76384 & 0.00003 & 0.09274 & 6.76414 & -4.98508 & 0.09299 & 0.00055 & -4.98426 \\
\hline 12 & Hard & $Y$ & 0.09275 & 0.00000 & 0.00000 & 0.09296 & 6.76552 & 0.00002 & 0.09288 & 6.76926 & -4.99236 & 0.09336 & 0.00337 & -4.99945 \\
\hline 13 & Hard & $\mathrm{N}$ & 0.09346 & 0.00000 & 0.00000 & 0.09348 & 6.76347 & -0.00027 & 0.09275 & 6.76425 & -4.99519 & 0.09309 & 0.00055 & -4.99282 \\
\hline 14 & Soft & $Y$ & 0.09306 & 0.00000 & 0.00000 & 0.09317 & 6.76454 & 0.00002 & 0.09276 & 6.76461 & -4.98446 & 0.09316 & 0.00061 & -4.98 \\
\hline 15 & Soft & $Y$ & 0.09314 & 0.00000 & 0.00000 & 0.09278 & 6.76449 & 0.00007 & 0.09304 & 6.76472 & -4.98458 & 316 & 0073 & \\
\hline 16 & Soft & $\mathrm{N}$ & 0.09296 & 0.00000 & 0.00000 & 0.09317 & 6.76363 & -0.00007 & 0.09309 & 6.76415 & -4.98440 & 0.09305 & 0038 & \\
\hline 17 & Hard & $\mathrm{N}$ & 0.09300 & 0.00000 & 0.00000 & 0.09355 & 6.76733 & 0.00003 & 0.09241 & 6.76362 & -5.00452 & 0.09236 & -0.00255 & -4.99360 \\
\hline 18 & Hard & $\mathrm{N}$ & 0.09226 & 0.00000 & 0.00000 & 0.09295 & 6.76420 & 0.00000 & 0.09334 & 6.76658 & -4.99280 & 0.09322 & 0.00174 & -4.99989 \\
\hline 19 & Soft & $\mathrm{N}$ & 0.09338 & 0.00000 & 0.00000 & 0.09300 & 6.76434 & -0.00005 & 0.09297 & 6.76450 & -4.98455 & 0.09288 & -0.00008 & -4.98374 \\
\hline 20 & Hard & $\bar{Y}$ & 0.09337 & 0.00000 & 0.00000 & 0.09329 & 6.76407 & -0.00001 & 0.09307 & 6.76461 & -4.99120 & 0.09389 & -0.00001 & -4.99270 \\
\hline 21 & Soft & $\mathrm{Y}$ & 0.09301 & & & & 6.76399 & & & & & & & \\
\hline 22 & Hard & $\mathrm{Y}$ & 0.09270 & 0.00000 & 0.00000 & 0.09251 & 6.76472 & & 0.09268 & 6.76366 & -4.99727 & 0.09275 & -0.00206 & -4.99 \\
\hline 23 & Hard & $\mathrm{Y}$ & 0.09290 & 0.00000 & 0.00000 & 0.09205 & 6.76749 & 0.00004 & 0.09243 & 6.76508 & -4.99397 & 0.09280 & -0.00142 & -4.98929 \\
\hline 24 & Soft & $Y$ & 0.09323 & 0.00000 & 0.00000 & 0.09329 & 6.76333 & 0.00001 & 0.09289 & 6.76332 & -4.98425 & 0.09343 & 0.00044 & -4.98379 \\
\hline 25 & Hard & $\mathrm{N}$ & 0.09400 & 0.00000 & 0.00000 & 0.09299 & 6.76792 & 0.00003 & 0.09164 & 6.76444 & -4.99502 & 0.09266 & -0.00261 & -4.99337 \\
\hline 26 & Soft & $\mathrm{N}$ & 0.09286 & 0.00000 & 0.00000 & 0.09292 & 6.76438 & 0.00002 & 0.09298 & 6.76463 & -4.98472 & 0.09304 & 0.00026 & -4.98440 \\
\hline 27 & Soft & $\mathrm{N}$ & 0.09292 & 0.00000 & 0.00000 & 0.09298 & 6.76400 & -0.00005 & 0.09271 & 6.76453 & -4.98420 & 0.09319 & 0.00052 & -4.98372 \\
\hline 28 & Hard & $\mathrm{Y}$ & 0.09403 & 0.00000 & 0.00000 & 0.09219 & 6.76610 & & & & & & 0.00004 & \\
\hline 29 & Hard & $\mathrm{N}$ & 0.09326 & 0.00000 & 0.00000 & 0.09265 & 6.76508 & -0.00001 & 0.09308 & 6.76376 & -4.99609 & 0.09306 & -0.00072 & -4.99387 \\
\hline 30 & Soft & $\mathrm{Y}$ & 0.09320 & 0.00000 & 0.00000 & 0.09297 & 6.76471 & 0.00002 & 0.09277 & 6.76492 & -4.98401 & 0.09291 & 0.00029 & -4.98360 \\
\hline 31 & Soft & $Y$ & 0.09284 & 0.00000 & 0.00000 & 0.09290 & 6.76486 & 0.00004 & 0.09261 & 6.76496 & -4.98418 & 0.09313 & 0.00045 & -4.98390 \\
\hline 32 & Soft & $\mathrm{N}$ & 0.09293 & 0.00000 & 0.00000 & 0.09283 & 6.76483 & 0.00009 & 0.09268 & 6.76506 & -4.98463 & 0.09040 & 0.00052 & -4.98440 \\
\hline
\end{tabular}

Table 4. Hole Location

\begin{tabular}{|c|c|c|c|c|c|c|c|c|c|c|}
\hline \multicolumn{11}{|c|}{ Analysis } \\
\hline \multicolumn{5}{|c|}{ Hole Diameter Deviations from Drilled } & \multicolumn{3}{|c|}{$\mathrm{X}$ axis PPM Distortion } & \multicolumn{3}{|c|}{$\mathrm{Y}$ axis PPM Distortion } \\
\hline UL & UR & LR & LL & Avg. & UL $\rightarrow$ UR & LL $\rightarrow$ LR & Avg. & $\mathrm{UL} \rightarrow \mathrm{LL}$ & UR $\rightarrow$ LR & Avg. \\
\hline-0.00094 & -0.00014 & -0.00067 & -0.00070 & -0.00061 & 304.51 & 22.17 & 163.34 & 1153.46 & 1845.54 & 1499.50 \\
\hline-0.00069 & -0.00039 & -0.00052 & -0.00050 & -0.00053 & -116.78 & -51.74 & -84.26 & 1346.04 & 1121.36 & 1233.70 \\
\hline-0.00029 & -0.00045 & -0.00068 & -0.00077 & -0.00055 & -162.60 & -68.00 & -115.30 & -54.16 & -84.25 & -69.21 \\
\hline-0.00055 & -0.00091 & -0.00072 & -0.00107 & -0.00081 & 19.22 & 141.91 & 80.56 & 1632.90 & 858.58 & 1245.74 \\
\hline-0.00057 & -0.00036 & -0.00052 & -0.00052 & -0.00049 & -143.39 & -167.04 & -155.21 & 12.04 & -184.55 & -86.26 \\
\hline-0.00037 & -0.00050 & -0.00056 & -0.00085 & -0.00057 & -301.55 & -220.25 & -260.90 & 1041.12 & 1861.58 & 1451.35 \\
\hline-0.00035 & -0.00028 & -0.00066 & -0.00067 & -0.00049 & -325.20 & -339.99 & -332.59 & 850.55 & 730.19 & 790.37 \\
\hline-0.00059 & -0.00044 & -0.00042 & -0.00061 & -0.00051 & -199.56 & -168.51 & -184.04 & -150.45 & -234.70 & -192.58 \\
\hline-0.00047 & -0.00023 & -0.00059 & -0.00077 & -0.00051 & 42.87 & -32.52 & 5.17 & 2082.25 & 716.15 & 1399.20 \\
\hline-0.00067 & -0.00031 & -0.00098 & -0.00073 & -0.00067 & -165.56 & -130.08 & -147.82 & 20.06 & -48.14 & -14.04 \\
\hline-0.00056 & -0.00041 & -0.00076 & -0.00051 & -0.00056 & -171.47 & -208.43 & -189.95 & 16.05 & -142.43 & -63.19 \\
\hline-0.00075 & -0.00054 & -0.00062 & -0.00014 & -0.00051 & 76.87 & 131.56 & 104.21 & 1476.43 & 2902.71 & 2189.57 \\
\hline-0.00004 & -0.00002 & -0.00075 & -0.00041 & -0.00031 & -226.16 & $\begin{array}{l}-192.17 \\
\end{array}$ & -209.16 & 2044.13 & 1514.54 & 1779.34 \\
\hline-0.00044 & -0.00033 & -0.00074 & -0.00034 & -0.00046 & -68.00 & -147.82 & $\begin{array}{l}-107.91 \\
\end{array}$ & -108.32 & -164.49 & -136.41 \\
\hline-0.00036 & -0.00072 & -0.00046 & -0.00034 & -0.00047 & -75.39 & -149.30 & -112.34 & -84.25 & -190.57 & -137.41 \\
\hline-0.00054 & -0.00033 & -0.00041 & -0.00045 & -0.00043 & -202.51 & -181.82 & -192.17 & -120.36 & -230.69 & -175.53 \\
\hline-0.00050 & 0.00005 & -0.00109 & -0.00114 & -0.00067 & 344.42 & 172.95 & 258.68 & 3915.75 & 1731.19 & 2823.47 \\
\hline-0.00124 & -0.00055 & -0.00016 & -0.00028 & -0.00056 & -118.26 & -23.65 & -70.95 & 1564.69 & 2986.96 & 2275.83 \\
\hline-0.00012 & -0.00050 & -0.00053 & -0.00062 & -0.00044 & -97.56 & -62.08 & -79.82 & -90.27 & -262.79 & -176.53 \\
\hline-0.00013 & -0.00021 & -0.00043 & & -0.00010 & & -56.17 & -96.82 & & 1542.63 & \\
\hline-0.00049 & -0.00045 & -0.00079 & -0.00065 & -0.00060 & -149.30 & -76.87 & -113.08 & -38.11 & -234.70 & -136.41 \\
\hline-0.00080 & -0.00099 & -0.00082 & -0.00075 & -0.00084 & -41.39 & 106.43 & 32.52 & 2461.38 & 1436.31 & 1948.85 \\
\hline-0.00060 & -0.00145 & -0.00107 & -0.00070 & -0.00096 & 368.07 & 221.73 & 294.90 & 1799.40 & 868.61 & 1334.00 \\
\hline-0.00027 & -0.00021 & -0.00061 & -0.00007 & -0.00029 & -246.86 & -313.38 & -280.12 & -150.45 & -240.72 & -195.59 \\
\hline 0.00050 & -0.00051 & -0.00186 & -0.00084 & -0.00068 & 431.63 & 303.03 & 367.33 & 2010.03 & 1685.06 & 1847.54 \\
\hline-0.00064 & -0.00058 & -0.00052 & -0.00046 & -0.00055 & -91.65 & -93.13 & -92.39 & -56.17 & -116.35 & -86.26 \\
\hline-0.00058 & -0.00052 & -0.00079 & -0.00031 & -0.00055 & -147.82 & -146.34 & -147.08 & -160.48 & -266.80 & -213.64 \\
\hline 0.00053 & -0.00131 & -0.00043 & -0.00059 & -0.00045 & 162.60 & 288.25 & 225.42 & 2369.11 & 1995.99 & 2182.55 \\
\hline-0.00024 & -0.00085 & -0.00042 & -0.00044 & -0.00049 & 11.83 & -76.87 & -32.52 & 2224.67 & 1777.33 & 2001.00 \\
\hline-0.00030 & -0.00053 & -0.00073 & -0.00059 & -0.00054 & -42.87 & -54.69 & -48.78 & -198.60 & -276.83 & -237.71 \\
\hline-0.00066 & -0.00060 & -0.00089 & -0.00037 & -0.00063 & -20.69 & -72.43 & -46.56 & -164.49 & -212.64 & -188.57 \\
\hline-0.00057 & \begin{tabular}{|l|}
-0.00067 \\
\end{tabular} & -0.00082 & -0.00310 & -0.00129 & -25.13 & -68.00 & -46.56 & -74.22 & -102.31 & -88.26 \\
\hline
\end{tabular}

Table 5. Hole Analysis 


\begin{tabular}{|c|c|c|c|c|c|c|c|c|c|c|c|c|}
\hline $\begin{array}{l}\text { Std } \\
\text { Order }\end{array}$ & $\begin{array}{l}\text { Run } \\
\text { Order } \\
\end{array}$ & $\begin{array}{l}\text { Pt } \\
\text { Type }\end{array}$ & Blocks & Stackup & Bake & Clean & Dia & $\mathrm{X}$ Axis & Y Axis & $\begin{array}{l}\text { Method } \\
2 \mathrm{~A} \\
\end{array}$ & $\begin{array}{l}\text { Method } \\
\text { 2B } \\
\end{array}$ & $\begin{array}{l}\text { Method } \\
1 \\
\end{array}$ \\
\hline 6 & 1 & 1 & 1 & Rubber & No Bake & Clean & -0.0006 & 163.34 & 1499.5 & 1 & 0 & 8 \\
\hline 7 & 2 & 1 & 1 & Rubber & No Bake & No Clean & -0.0005 & -84.26 & 1233.7 & 0 & 0 & 0 \\
\hline 15 & 3 & 1 & 1 & PacoPad & No Bake & No Clean & -0.0006 & -115.3 & -69.21 & 15 & 11 & 75 \\
\hline 4 & 4 & 1 & 1 & Rubber & Bake & No Clean & -0.0008 & 80.56 & 1245.74 & 1 & 0 & 21 \\
\hline 11 & 5 & 1 & 1 & PacoPad & Bake & No Clean & -0.0005 & -155.21 & -86.26 & 0 & 0 & 36 \\
\hline 2 & 6 & 1 & 1 & Rubber & Bake & Clean & -0.0006 & -260.9 & 1451.35 & 4 & 0 & 24 \\
\hline 3 & 7 & 1 & 1 & Rubber & Bake & No Clean & -0.0005 & -332.59 & 790.37 & 0 & 0 & 6 \\
\hline 9 & 8 & 1 & 1 & PacoPad & Bake & Clean & -0.0005 & -184.04 & -192.58 & 1 & 0 & 38 \\
\hline 5 & 9 & 1 & 1 & Rubber & No Bake & Clean & -0.0005 & 5.17 & 1399.2 & 0 & 0 & 0 \\
\hline 16 & 10 & 1 & 1 & PacoPad & No Bake & No Clean & -0.0007 & -147.82 & -14.04 & 14 & 11 & 75 \\
\hline 13 & 11 & 1 & 1 & PacoPad & No Bake & Clean & -0.0006 & -189.95 & -63.19 & 14 & 11 & 75 \\
\hline 1 & 12 & 1 & 1 & Rubber & Bake & Clean & -0.0005 & 104.21 & 2189.57 & 5 & 0 & 72 \\
\hline 8 & 13 & 1 & 1 & Rubber & No Bake & No Clean & -0.0003 & -209.16 & 1779.34 & 0 & 0 & 8 \\
\hline 10 & 14 & 1 & 1 & PacoPad & Bake & Clean & -0.0005 & -107.91 & -136.41 & 1 & 0 & 17 \\
\hline 12 & 15 & 1 & 1 & PacoPad & Bake & No Clean & -0.0005 & -112.34 & -137.41 & 0 & 0 & 20 \\
\hline 14 & 16 & 1 & 1 & PacoPad & No Bake & Clean & -0.0004 & -192.17 & -175.53 & 13 & 8 & 75 \\
\hline 22 & 17 & 1 & 2 & Rubber & No Bake & Clean & -0.0007 & 258.68 & 2823.47 & 2 & 0 & 29 \\
\hline 23 & 18 & 1 & 2 & Rubber & No Bake & No Clean & -0.0006 & -70.95 & 2275.83 & 1 & 0 & 12 \\
\hline 31 & 19 & 1 & 2 & PacoPad & No Bake & No Clean & -0.0004 & -79.82 & -176.53 & 14 & 9 & 75 \\
\hline 20 & 20 & 1 & 2 & Rubber & Bake & No Clean & -0.0001 & -96.82 & 1393.18 & 0 & 0 & 8 \\
\hline 27 & 21 & 1 & 2 & PacoPad & Bake & No Clean & -0.0006 & -113.08 & -136.41 & 0 & 0 & 24 \\
\hline 18 & 22 & 1 & 2 & Rubber & Bake & Clean & -0.0008 & 32.52 & 1948.85 & 4 & 0 & 49 \\
\hline 19 & 23 & 1 & 2 & Rubber & Bake & No Clean & -0.001 & 294.9 & 1334 & 1 & 0 & 29 \\
\hline 25 & 24 & 1 & 2 & PacoPad & Bake & Clean & -0.0003 & -280.12 & -195.59 & 0 & 0 & 38 \\
\hline 21 & 25 & 1 & 2 & Rubber & No Bake & Clean & -0.0007 & 367.33 & 1847.54 & 1 & 0 & 15 \\
\hline 32 & 26 & 1 & 2 & PacoPad & No Bake & No Clean & -0.0006 & -92.39 & -86.26 & 13 & 11 & 75 \\
\hline 29 & 27 & 1 & 2 & PacoPad & No Bake & Clean & -0.0006 & -147.08 & -213.64 & 14 & 12 & 75 \\
\hline 17 & 28 & 1 & 2 & Rubber & Bake & Clean & -0.0005 & 225.42 & 2182.55 & 7 & 0 & 45 \\
\hline 24 & 29 & 1 & 2 & Rubber & No Bake & No Clean & -0.0005 & -32.52 & 2001 & 0 & 0 & 8 \\
\hline 26 & 30 & 1 & 2 & PacoPad & Bake & Clean & -0.0005 & -48.78 & -237.71 & 0 & 0 & 28 \\
\hline 28 & 31 & 1 & 2 & PacoPad & Bake & No Clean & -0.0006 & -46.56 & -188.57 & 0 & 0 & 24 \\
\hline 30 & 32 & 1 & 2 & PacoPad & No Bake & Clean & -0.0013 & -46.56 & -88.26 & 15 & 11 & 75 \\
\hline
\end{tabular}

Table 6. DoE Variables and Results 


\title{
Appendix B
}

\author{
Phase Two Data
}

\section{Hole location data}

A department's CMM hole location data is shown below. The post laminate measurements were taken after lamination.

$\mathrm{PPM}=\mathrm{ABS}(\mathrm{A}-(\mathrm{ABS}(\mathrm{B}-\mathrm{C}))) / \mathrm{A}) \times 1000000$

$\mathrm{A}=$ Delta of nominal data

$\mathrm{B}=$ hole location

$\mathrm{C}=$ hole location

$\mathrm{B}-\mathrm{C}=$ distance between two holes

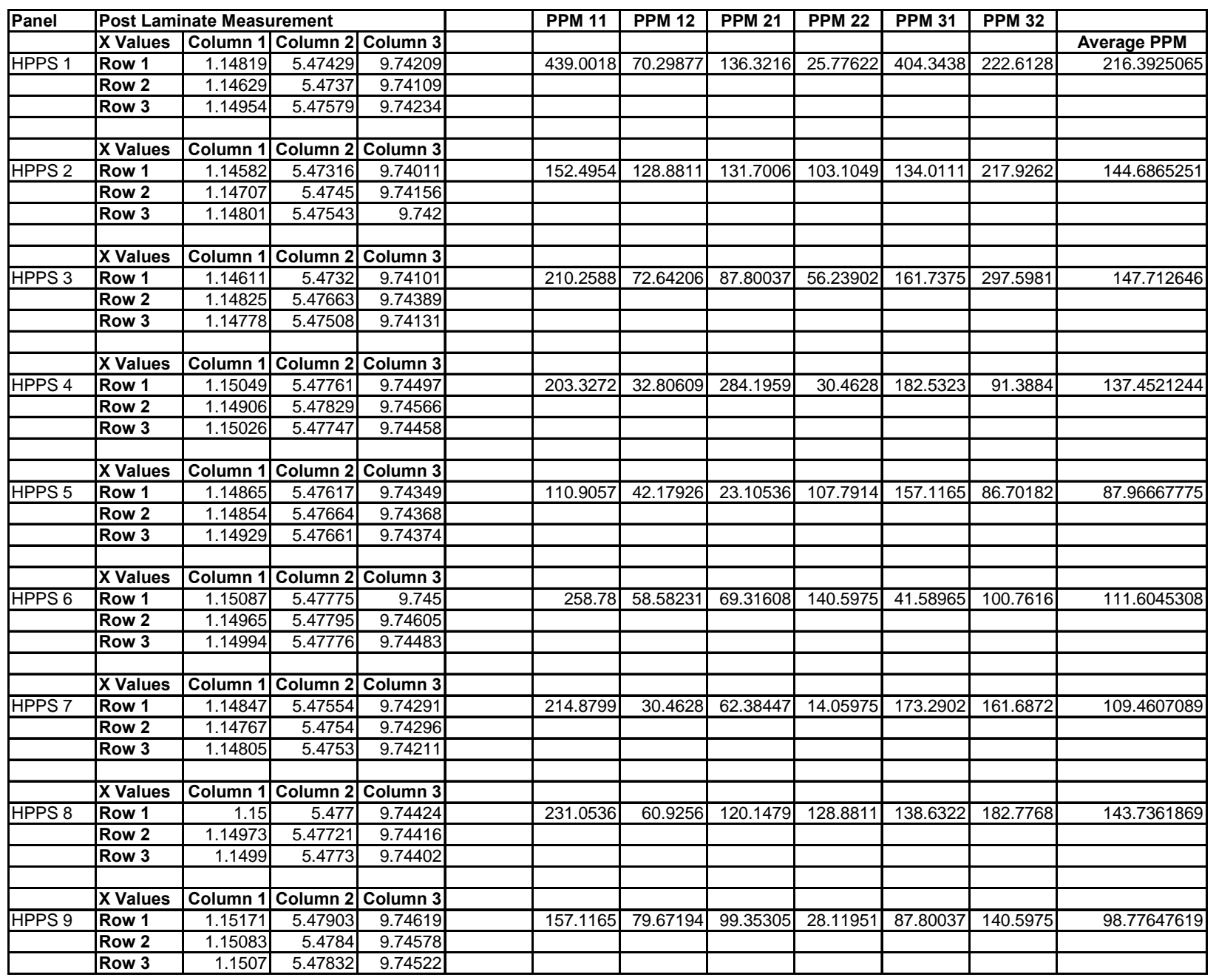

\section{Table 7. Post Laminate Hole Location X Values}




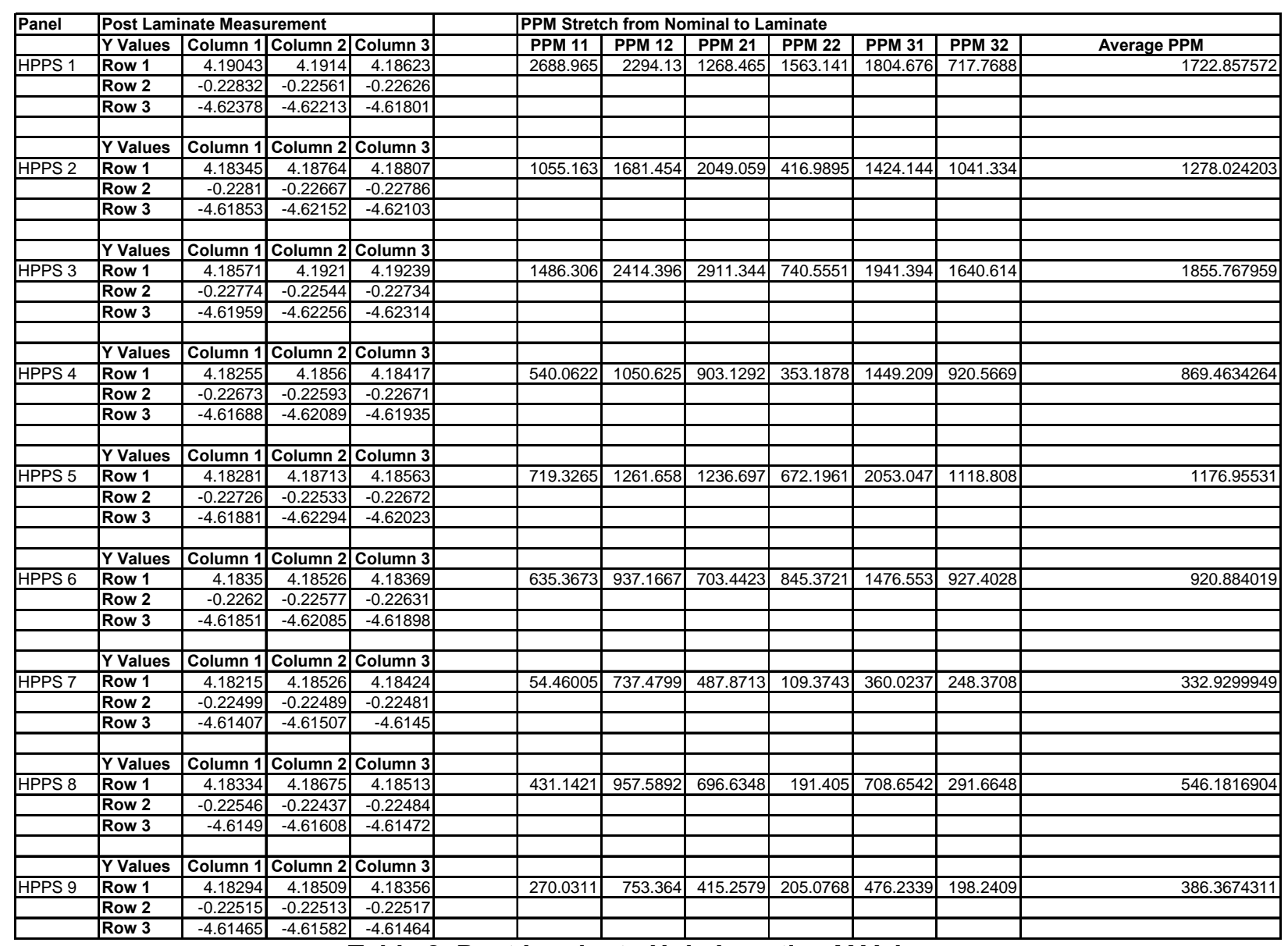

Table 8. Post Laminate Hole Location Y Values 


\section{Adhesion Quality Data}

The area around each land was evaluated for solder wicking/discoloration and if any was discovered the maximum ingress was measured and recorded. The measurement recorded was the linear distance under the coverlay not an attempt to quantify the total area covered by any solder wicking/discoloration. The data shown below signifies discoloration since solder wicking was found only on one panel four land and one panel five land.

The green column in the table below displays the additive total of land discoloration per part. This total was used to rank each part and then rank each panel.

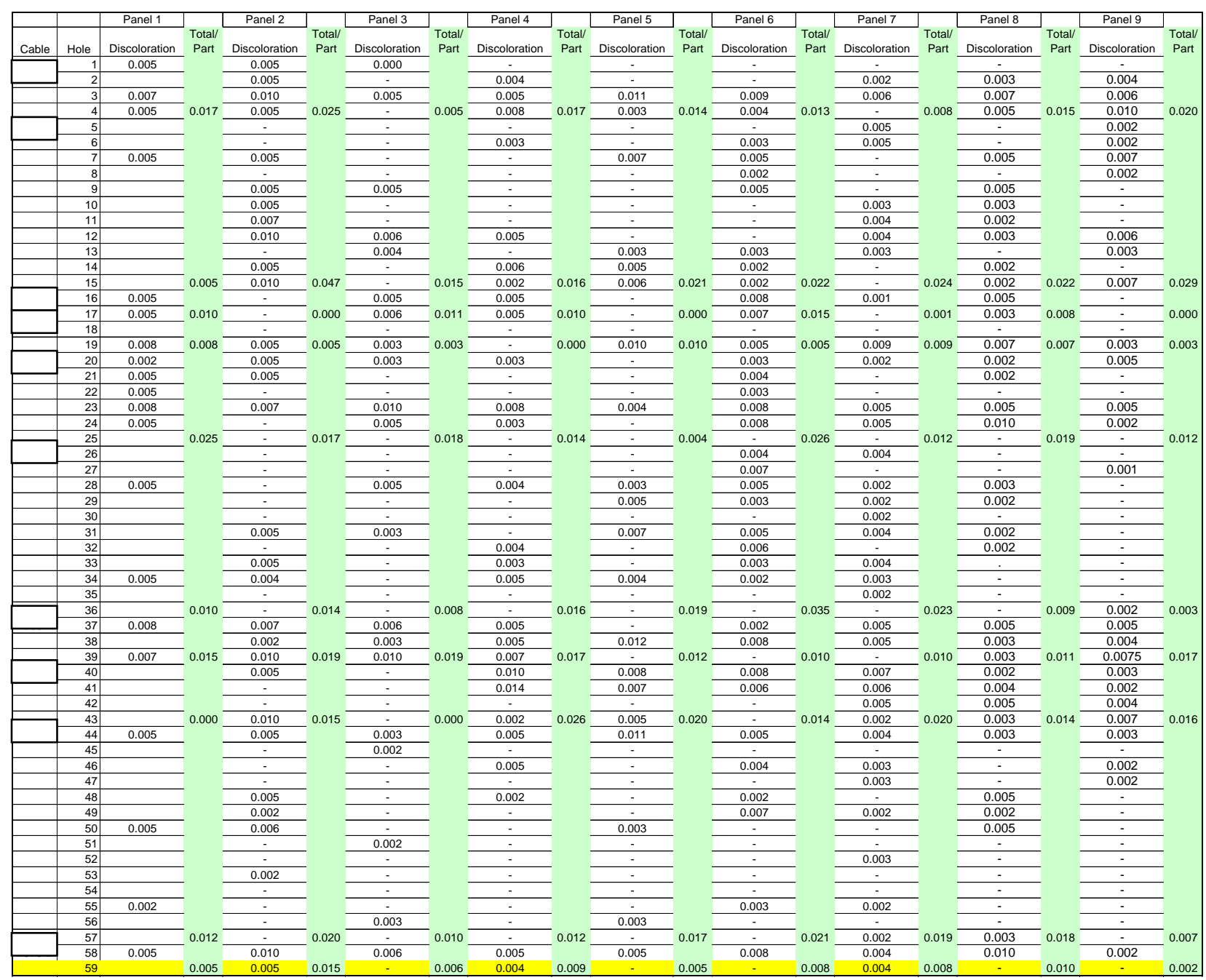

Table 9. Adhesion Quality Data 


\section{One-Way ANOVA: PPM X Versus Pressure}

$\begin{array}{lrrrrr}\text { Source } & \text { DF } & \text { SS } & \text { MS } & \text { F } & P \\ \text { Pressure } & 2 & 904 & 452 & 1.13 & 0.382 \\ \text { Error } & 6 & 2392 & 399 & & \\ \text { Total } & 8 & 3296 & & & \\ \text { S }=19.97 & \text { R-Sq }=27.44 \% & \text { R-Sq }(\text { adj })=3.25 \%\end{array}$

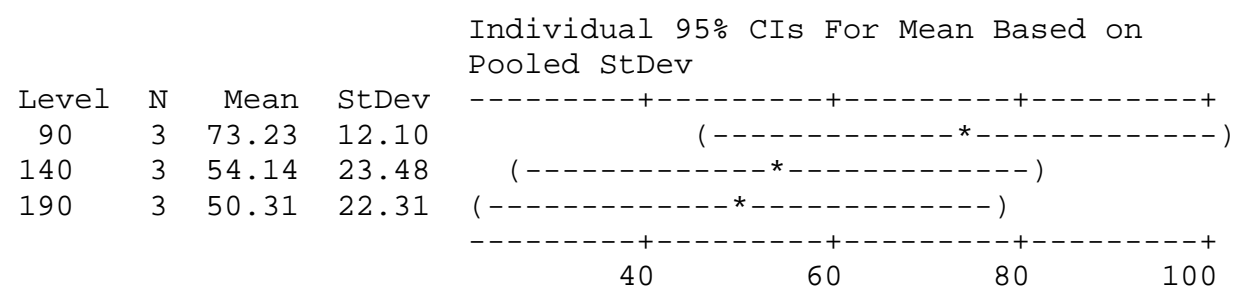

Pooled StDev $=19.97$

One-Way ANOVA: PPM Y Versus Pressure

$\begin{array}{lrrrrc}\text { Source } & \text { DF } & \text { SS } & \text { MS } & F & P \\ \text { Pressure } & 2 & 1664394 & 832197 & 22.27 & 0.002 \\ \text { Error } & 6 & 224243 & 37374 & & \\ \text { Total } & 8 & 1888637 & & & \\ S=193.3 & \text { R-Sq }=88.13 \% & \text { R-Sq }(\text { adj })=84.17 \%\end{array}$

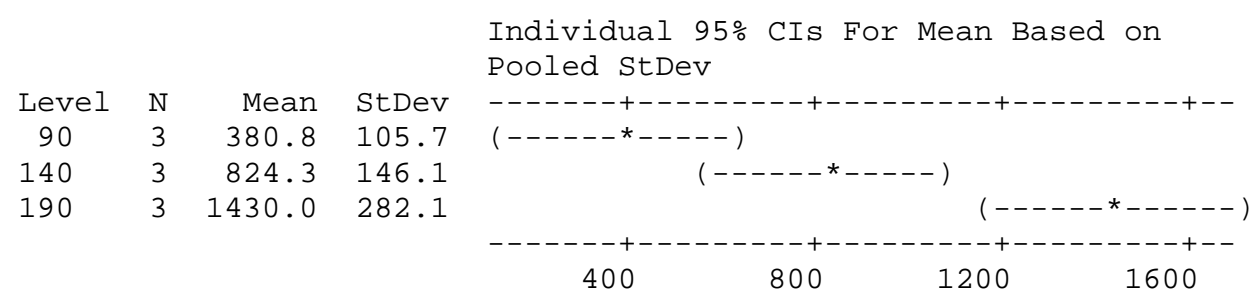

Pooled StDev $=193.3$

\section{One-Way ANOVA: Discoloration Versus Pressure}

$\begin{array}{lrrrrr}\text { Source } & \text { DF } & \text { SS } & \text { MS } & \text { F } & P \\ \text { Pressure } & 2 & 594 & 297 & 0.33 & 0.733 \\ \text { Error } & 6 & 5448 & 908 & & \\ \text { Total } & 8 & 6042 & & & \\ \text { S }=30.13 & \text { R-Sq }=9.82 \% & \text { R-Sq }(\text { adj })=0.00 \%\end{array}$

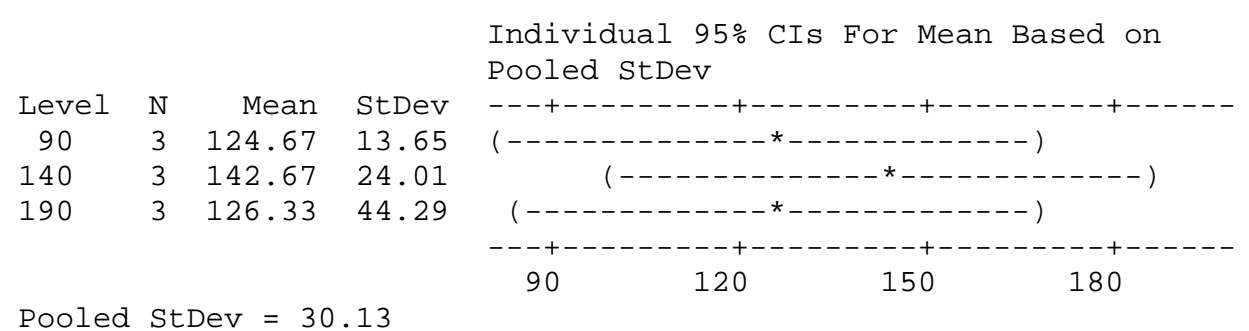




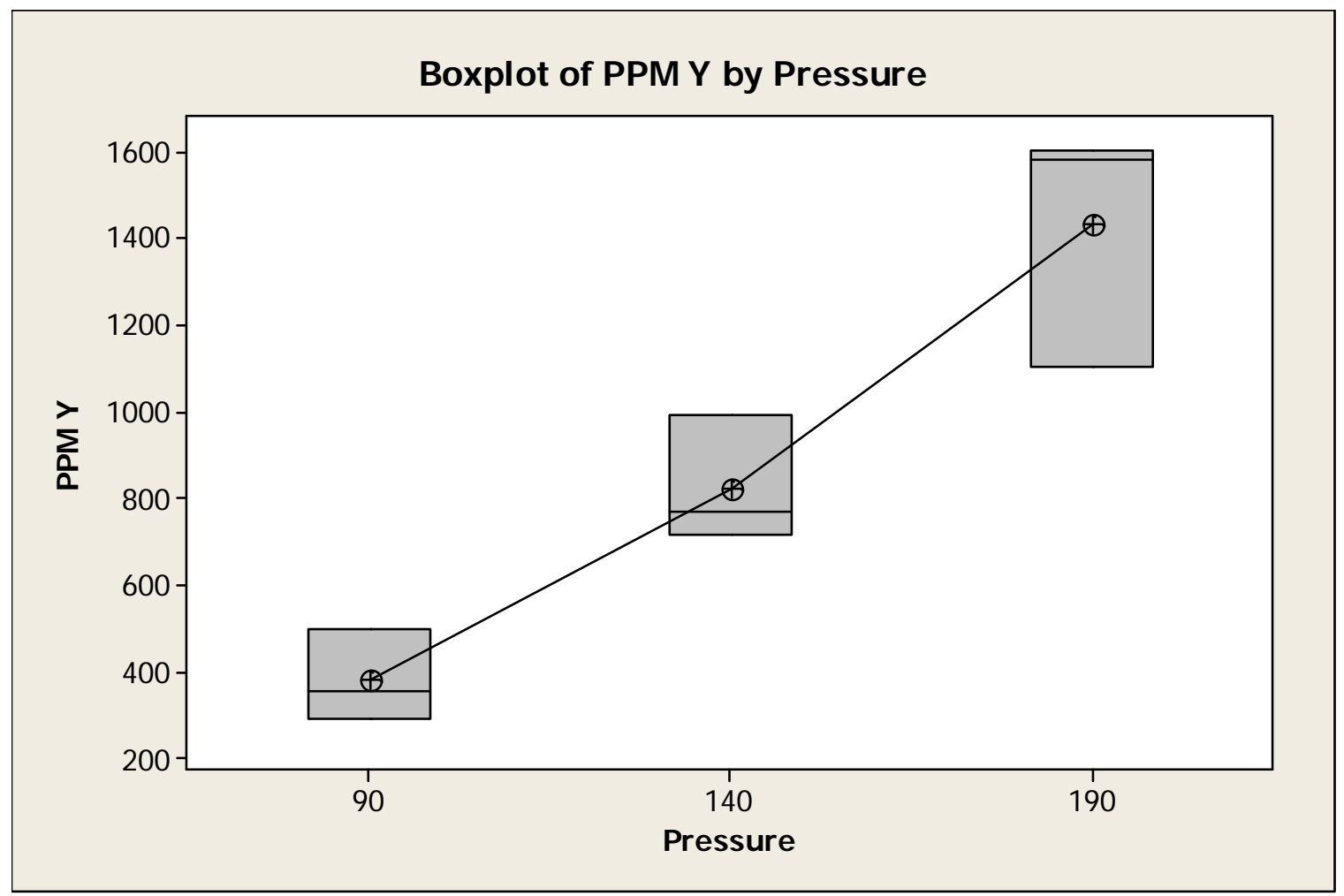

Figure 33. Boxplot of PPM Y by Pressure

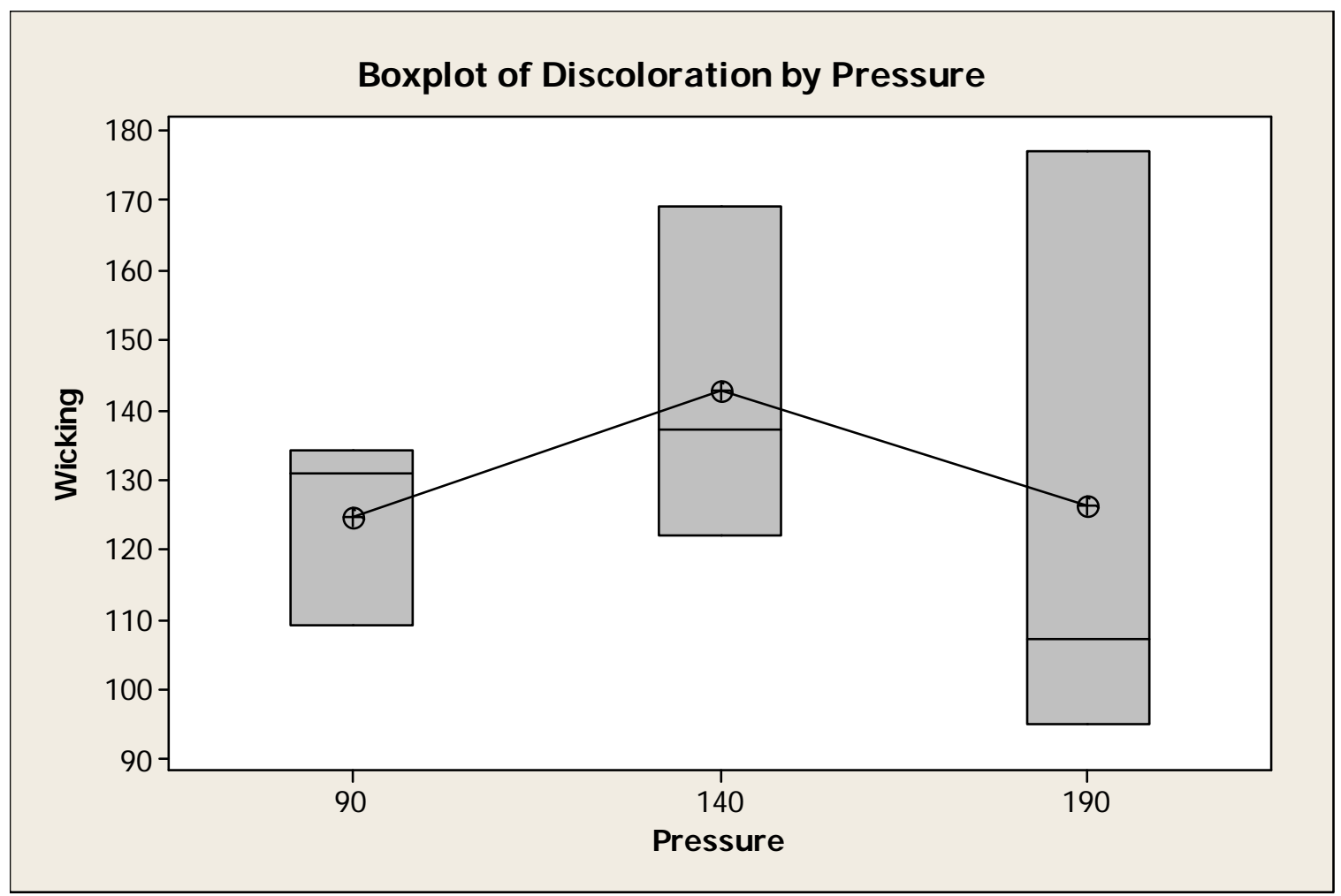

Figure 34. Boxplot of Discoloration by Pressure 


\section{Appendix C \\ Phase Three Data}

\section{Sample (Two Ounce Copper Laminate)}

\begin{tabular}{|c|c|c|c|c|c|c|c|c|c|c|c|c|}
\hline Panel & Post Lam & nation Mea & isurements & & & PPM 11 & PPM 12 & PPM 21 & PPM 22 & PPM 31 & PPM 32 & \\
\hline & $X$ Values & Column 1 & \begin{tabular}{|l|} 
Column 2 \\
\end{tabular} & Column 3 & & & & & & & & Average PPM \\
\hline SPPS 1 & Row 1 & 1.14843 & \begin{tabular}{|r|}
5.47607 \\
\end{tabular} & \begin{tabular}{|l|}
9.74369 \\
\end{tabular} & & 83.17930 & 28.11951 & 94.73198 & 7.02988 & 73.93715 & 60.92560 & 57.98724 \\
\hline & Row 2 & 1.14848 & 5.47607 & 9.7436 & & & & & & & & \\
\hline & Row 3 & 1.14851 & 5.47619 & 9.74395 & & & & & & & & \\
\hline & & & & & & & & & & & & \\
\hline & $X$ Values & Column 1 & \begin{tabular}{|l|} 
Column 2 \\
\end{tabular} & \begin{tabular}{|l|} 
Column 3 \\
\end{tabular} & & & & & & & & \\
\hline SPPS 2 & Row 1 & 1.14883 & \begin{tabular}{|r|}
5.4766 \\
\end{tabular} & \begin{tabular}{|r|}
9.7439 \\
\end{tabular} & & 53.14233 & 46.86585 & 115.52680 & 79.67194 & 20.79482 & 79.67194 & 65.94561 \\
\hline & Row 2 & 1.1481 & 5.4756 & 9.74276 & & & & & & & & \\
\hline & Row 3 & 1.14667 & 5.47476 & 9.74192 & & & & & & & & \\
\hline & & & & & & & & & & & & \\
\hline & $X$ Values & Column 1 & \begin{tabular}{|l|} 
Column 2 \\
\end{tabular} & Column 3 & & & & & & & & \\
\hline SPPS 3 & Row 1 & 1.14837 & \begin{tabular}{|l|}
5.47576 \\
\end{tabular} & \begin{tabular}{|r|}
9.74301 \\
\end{tabular} & & 140.94270 & 58.58231 & 145.56377 & 35.14938 & 39.27911 & 21.08963 & 73.43448 \\
\hline & Row 2 & 1.148 & 5.47537 & 9.74272 & & & & & & & & \\
\hline & Row 3 & 1.14719 & 5.47502 & 9.74243 & & & & & & & & \\
\hline & & & & & & & & & & & & \\
\hline & $X$ Values & Column 1 & \begin{tabular}{|l|} 
Column 2 \\
\end{tabular} & Column 3 & & & & & & & & \\
\hline SPPS 4 & Row 1 & 1.14885 & \begin{tabular}{|l|}
5.47619 \\
\end{tabular} & \begin{tabular}{|r|}
9.74338 \\
\end{tabular} & & 152.49538 & 72.64206 & 288.81701 & 35.14938 & 177.91128 & 65.61219 & 132.10455 \\
\hline & Row 2 & 1.14934 & 5.47609 & 9.74374 & & & & & & & & \\
\hline & Row 3 & 1.14894 & 5.47617 & 9.74339 & & & & & & & & \\
\hline & & & & & & & & & & & & \\
\hline & $X$ Values & Column 1 & \begin{tabular}{|l|} 
Column 2 \\
\end{tabular} & \begin{tabular}{|l|} 
Column 3 \\
\end{tabular} & & & & & & & & \\
\hline SPPS 5 & Row 1 & 1.1497 & \begin{tabular}{|l|}
5.47737 \\
\end{tabular} & \begin{tabular}{|l|}
9.74437 \\
\end{tabular} & & 76.24769 & 117.16462 & $\begin{array}{l}73.93715 \\
\end{array}$ & 124.19449 & 60.07394 & 77.32865 & 88.15776 \\
\hline & Row 2 & 1.14981 & 5.47749 & 9.74446 & & & & & & & & \\
\hline & Row 3 & 1.14928 & 5.47702 & 9.74419 & & & & & & & & \\
\hline & & & & & & & & & & & & \\
\hline & $X$ Values & Column 1 & \begin{tabular}{|l|} 
Column 2 \\
\end{tabular} & \begin{tabular}{|l|} 
Column 3 \\
\end{tabular} & & & & & & & & \\
\hline SPPS 6 & Row 1 & 1.15045 & \begin{tabular}{|l|}
5.47811 \\
\end{tabular} & \begin{tabular}{|l|}
9.74531 \\
\end{tabular} & & .78.55823 & 70.29877 & 18.48429 & 67.95548 & 9.24214 & 4.68658 & 41.53758 \\
\hline & Row 2 & 1.14933 & 5.47725 & 9.74446 & & & & & & & & \\
\hline & Row 3 & 1.14917 & 5.47721 & 9.74469 & & & & & & & & \\
\hline & & & & & & & & & & & & \\
\hline & $X$ Values & Column 1 & Column 2 & Column 3 & & & & & & & & \\
\hline SPPS 7 & Row 1 & 1.1498 & \begin{tabular}{|r|}
5.47765 \\
\end{tabular} & \begin{tabular}{|r|}
9.74463 \\
\end{tabular} & & 34.65804 & 121.85120 & 34.65804 & 35.14938 & 48.52126 & 32.80609 & 51.27400 \\
\hline & Row 2 & 1.14903 & 5.47688 & 9.74423 & & & & & & & & \\
\hline & Row 3 & 1.14778 & 5.47599 & 9.74363 & & & & & & & & \\
\hline & $X$ Values & Column 1 & \begin{tabular}{|c|} 
Column 2 \\
\end{tabular} & Column 3 & & & & & 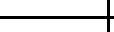 & -1 & -1 & -1 \\
\hline SPPS 8 & Row 1 & 1.14951 & \begin{tabular}{|l|}
5.47695 \\
\end{tabular} & \begin{tabular}{|l|}
9.74374 \\
\end{tabular} & & 129.39002 & 166.37376 & 78.55823 & 171.06034 & 50.83179 & 65.61219 & 110.30439 \\
\hline & Row 2 & 1.14913 & 5.47679 & 9.74356 & & & & & & & & \\
\hline & Row 3 & 1.14879 & 5.47657 & 9.74379 & & & & & & & & \\
\hline & & & & & & & & & & & & \\
\hline & $X$ Values & Column 1 & \begin{tabular}{|l|} 
Column 2 \\
\end{tabular} & \begin{tabular}{|l|} 
Column 3 \\
\end{tabular} & & & & & & & & \\
\hline SPPS 9 & Row 1 & 1.14876 & \begin{tabular}{|r|}
5.47624 \\
\end{tabular} & \begin{tabular}{|r|}
9.74352 \\
\end{tabular} & & 120.14787 & 51.55243 & 203.32717 & 110.13474 & 16.17375 & 112.47803 & 102.30233 \\
\hline & Row 2 & 1.1494 & 5.47652 & 9.74355 & & & & & & & & \\
\hline & Row 3 & 1.14846 & 5.47639 & 9.74341 & & & & & & & & \\
\hline & & & & & & & & & & & & \\
\hline SPPS 10 & $X$ Values & Column 1 & Column 2 & Column 3 & & & & & & & & \\
\hline SPPS 10 & Row 1 & 1.14845 & 5.47575 & 9.74265 & & 161.73752 & 140.59754 & 136.32163 & 149.97071 & 36.96858 & 63.26889 & 114.81081 \\
\hline & Row 2 & 1.14815 & 5.47556 & 9.74242 & & & & & & & & \\
\hline & Row 3 & 1.14758 & 5.47542 & 9.74265 & & & & & & & & \\
\hline & & & & & & & & & & & & \\
\hline & $X$ Values & Column 1 & \begin{tabular}{|l|} 
Column 2 \\
\end{tabular} & \begin{tabular}{|l|} 
Column 3 \\
\end{tabular} & & & & & & & & \\
\hline SPPS 11 & Row 1 & 1.14939 & \begin{tabular}{|l|}
5.47702 \\
\end{tabular} & 9.74454 & & 85.48983 & 4.68658 & 6.93161 & 56.23902 & 57.76340 & 49.20914 & 43.38660 \\
\hline & Row 2 & 1.14902 & 5.47699 & 9.74425 & & & & & & & & \\
\hline & Row 3 & 1.14847 & 5.47622 & 9.74393 & & & & & & & & \\
\hline & & & & & & & & & & & & \\
\hline & $X$ Values & Column 1 & \begin{tabular}{|l|} 
Column 2 \\
\end{tabular} & Column 3 & & & & & & & & \\
\hline SPPS 12 & Row 1 & 1.14919 & \begin{tabular}{|l|}
5.47643 \\
\end{tabular} & 9.74368 & & 175.60074 & 58.58231 & 120.14787 & 126.53779 & 147.87431 & 379.61336 & 168.05940 \\
\hline & Row 2 & 1.14808 & 5.47556 & 9.74252 & & & & & & & & \\
\hline & Row 3 & 1.14649 & 5.47385 & 9.74297 & & & & & & & & \\
\hline & & & & & & & & & & & & \\
\hline & $X$ Values & Column 1 & Column 2 & Column 3 & & & & & & & & \\
\hline SPPS 13 & Row 1 & 1.14893 & \begin{tabular}{|l|}
5.47613 \\
\end{tabular} & \begin{tabular}{|l|}
9.74259 \\
\end{tabular} & & 184.84288 & 243.70240 & 103.97412 & 117.16462 & 99.35305 & 49.20914 & 133.04104 \\
\hline & Row 2 & 1.14811 & 5.47566 & 9.74266 & & & & & & & & \\
\hline & Row 3 & 1.14808 & 5.47565 & 9.74294 & & & & & & & & \\
\hline & & & & & & & & & & & & \\
\hline & $X$ Values & Column 1 & \begin{tabular}{|l|} 
Column 2 \\
\end{tabular} & Column 3 & & & & & & & & \\
\hline SPPS 14 & Row 1 & 1.14993 & \begin{tabular}{|r|}
5.47681 \\
\end{tabular} & \begin{tabular}{|r|}
9.74381 \\
\end{tabular} & & 258.78004 & 117.16462 & 16.17375 & 157.00059 & 2.31054 & 7.02988 & 93.07657 \\
\hline & Row 2 & 1.14903 & 5.4771 & 9.74393 & & & & & & & & \\
\hline & Row 3 & 1.14821 & 5.47622 & 9.74375 & & & & & & & & \\
\hline & & & & & & & & & & & & \\
\hline & $X$ Values & Column 1 & \begin{tabular}{|l|} 
Column 2 \\
\end{tabular} & Column 3 & & & & & & & & \\
\hline SPPS 15 & Row 1 & 1.14692 & \begin{tabular}{|l|}
5.47426 \\
\end{tabular} & \begin{tabular}{|r|}
9.74143 \\
\end{tabular} & & 152.49538 & 77.32865 & 46.21072 & 135.91095 & 0.00000 & 84.35852 & 82.71737 \\
\hline & Row 2 & 1.14769 & 5.47549 & 9.74241 & & & & & & & & \\
\hline & Row 3 & 1.14871 & 5.47671 & 9.74385 & & & & & & & & \\
\hline & & & & & & & & & & & & \\
\hline & $X$ Values & Column 1 & \begin{tabular}{|l|} 
Column 2 \\
\end{tabular} & \begin{tabular}{|l|} 
Column 3 \\
\end{tabular} & & & & & & & & \\
\hline SPPS 16 & Row 1 & 1.14923 & 5.4764 & 9.7434 & & 191.77449 & 117.16462 & 217.19039 & 86.70182 & 154.80591 & 11.71646 & 129.89228 \\
\hline & Row 2 & 1.14968 & 5.47674 & 9.74387 & & & & & & & & \\
\hline & Row 3 & 1.1494 & 5.47673 & 9.74428| & & & & & & & & \\
\hline
\end{tabular}

Table 10. Panels 1-16 Rankings for X Values 


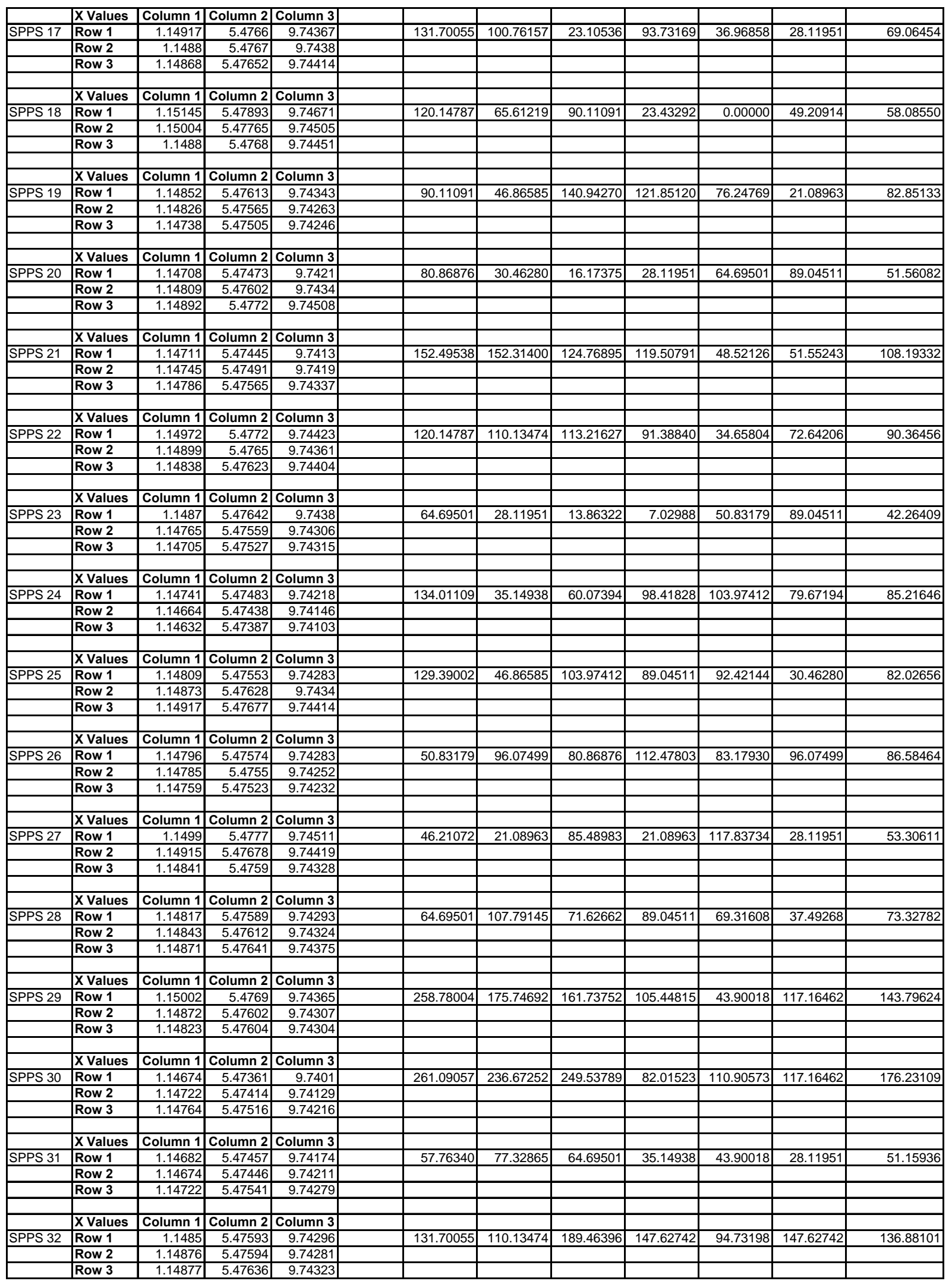

Table 11. Panels 17-32 Rankings for $X$ Values 


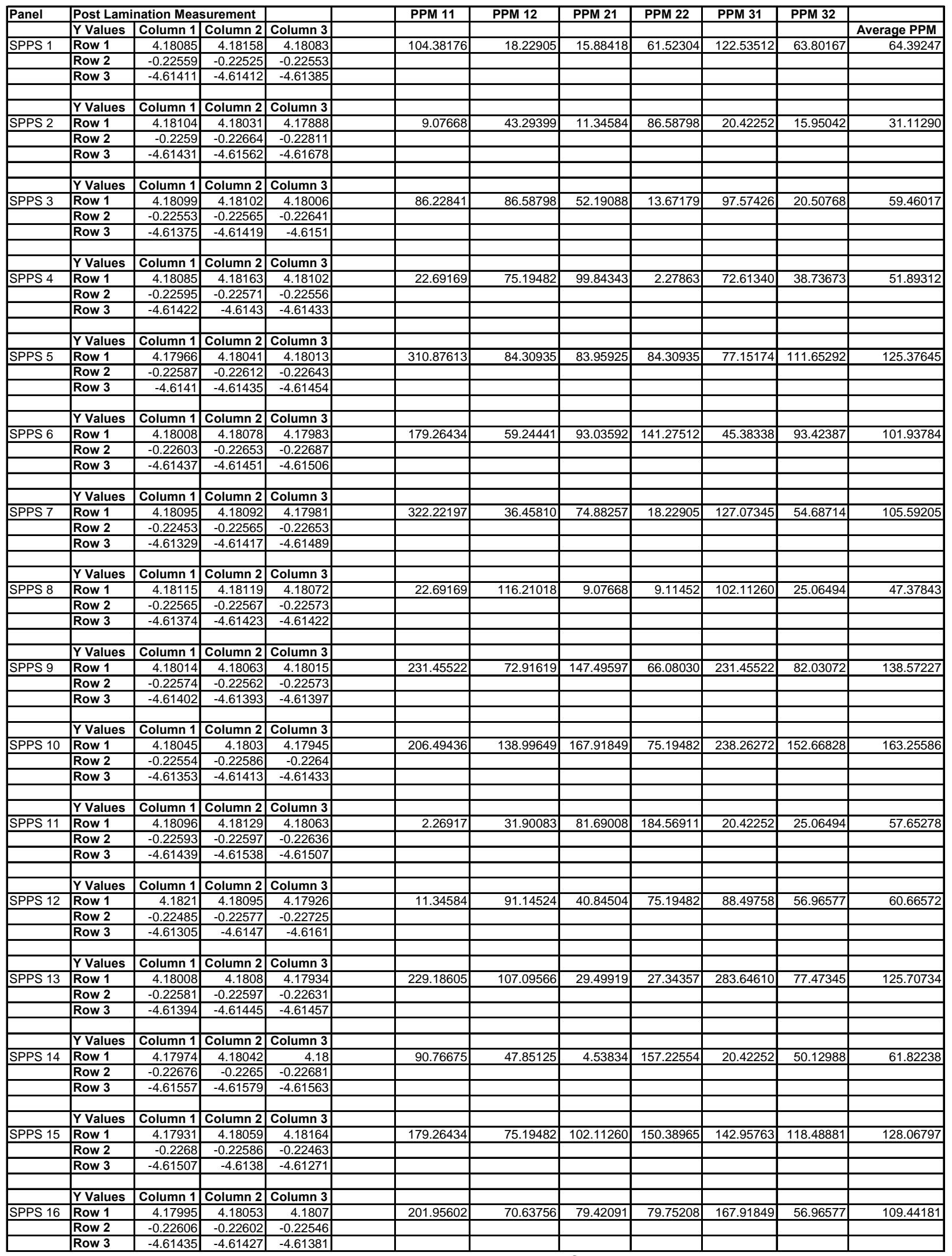

Table 12. Panels 1-16 Rankings for $Y$ Values 


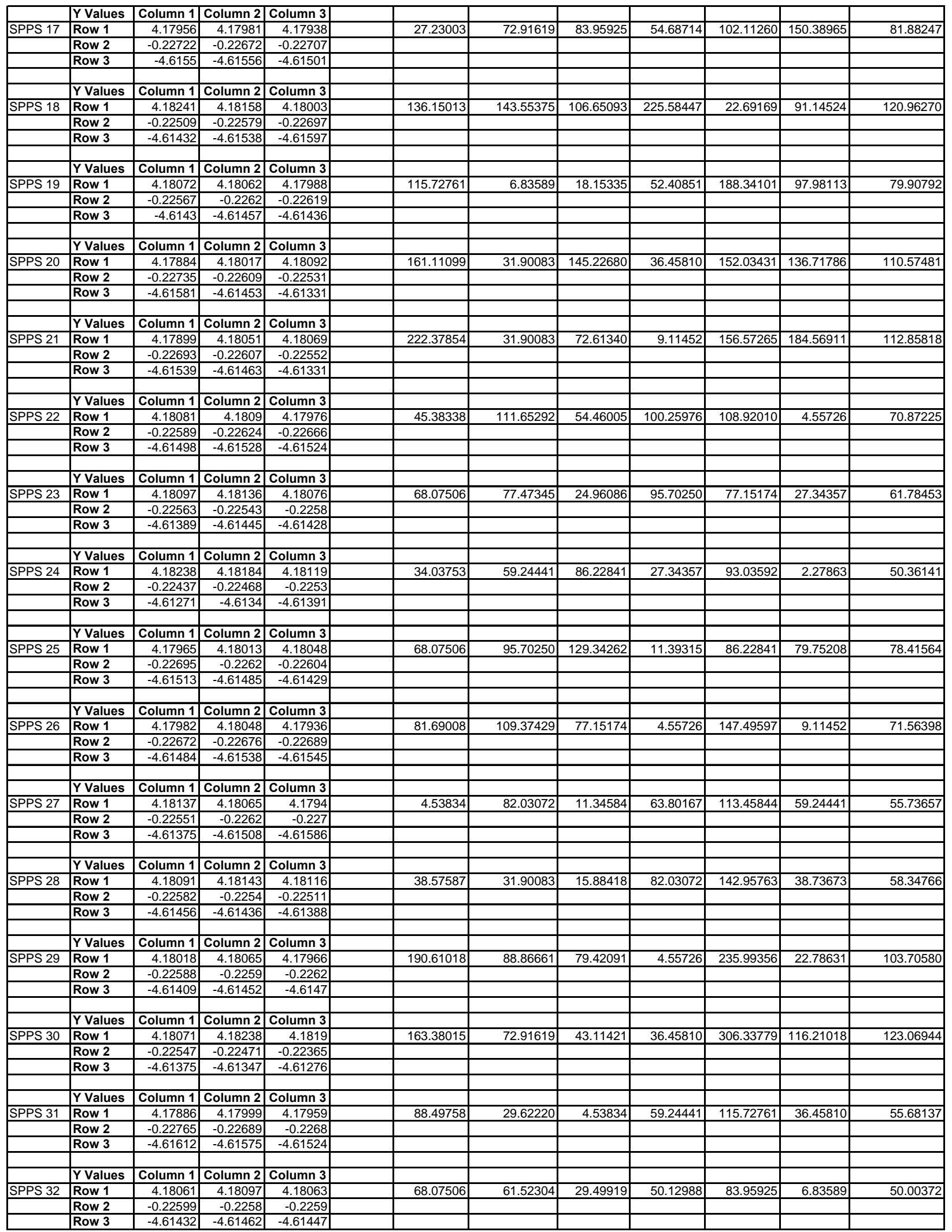

Table 13. Panels 17-32 Rankings for $Y$ Values 
The following five tables contain the actual measured amount of delamination/solder wicking on each land on each panel.

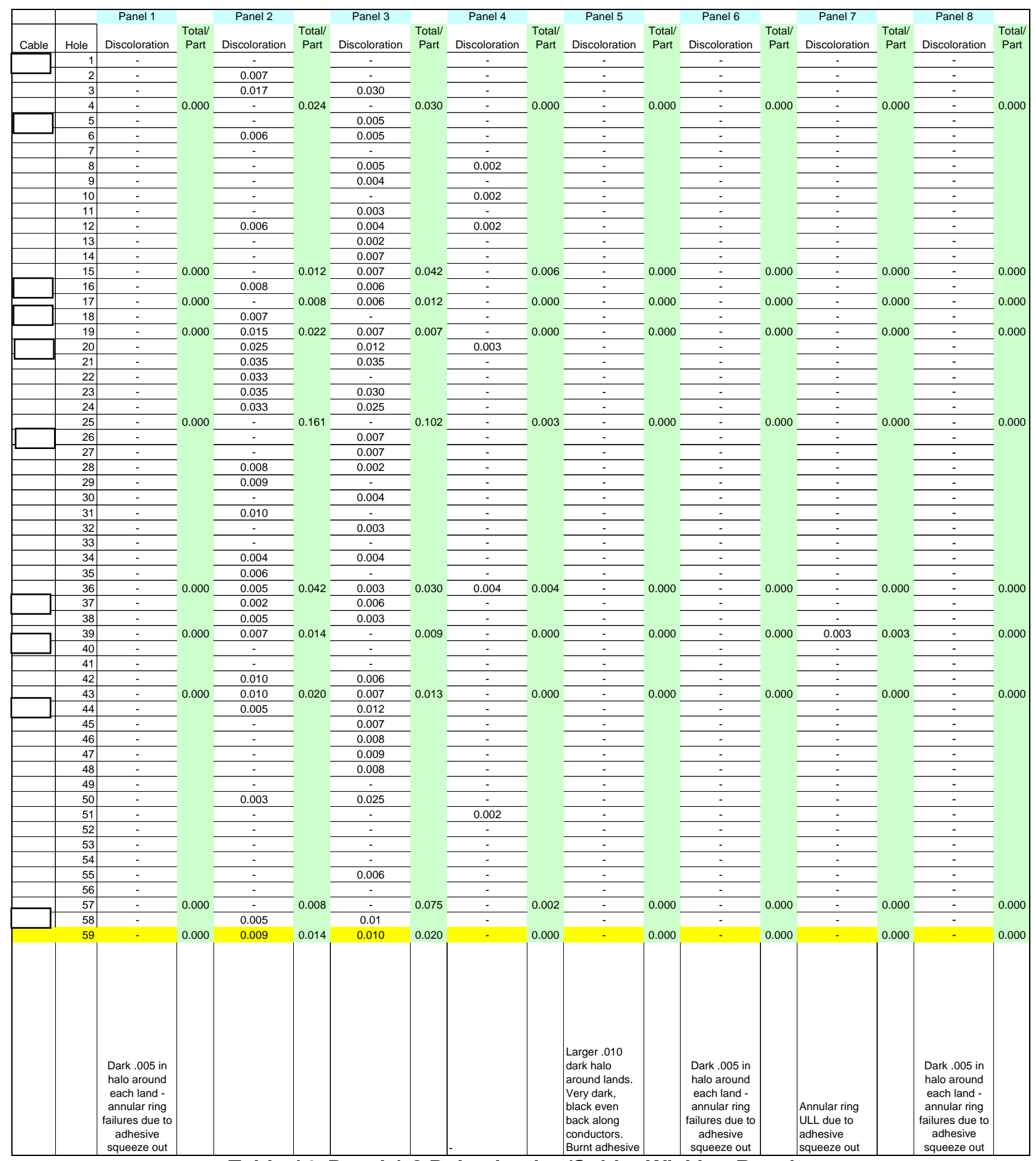

Table 14. Panel 1-8 Delamination/Solder Wicking Results 


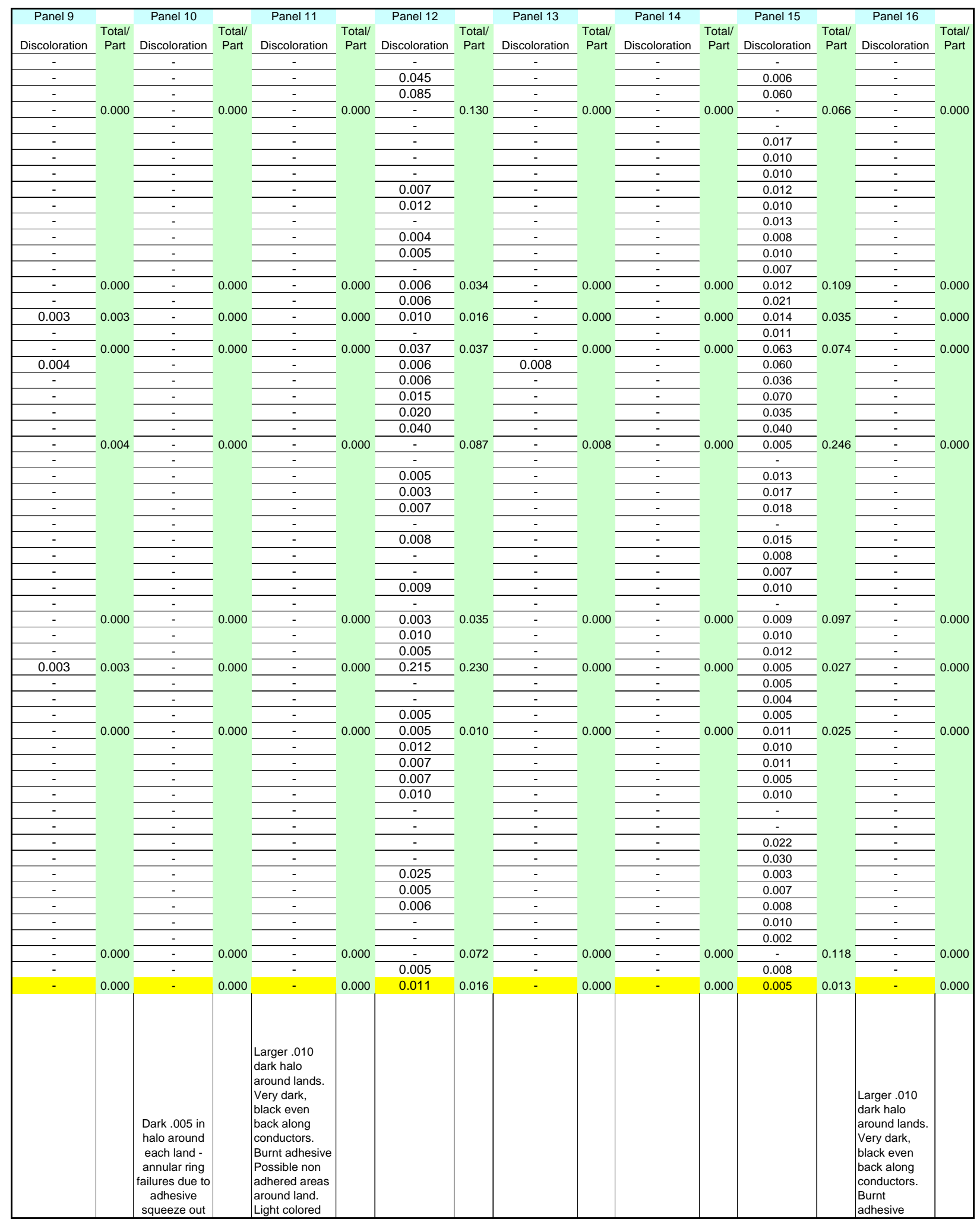

Table 15. Panel 9-16 Delamination/Solder Wicking Results 


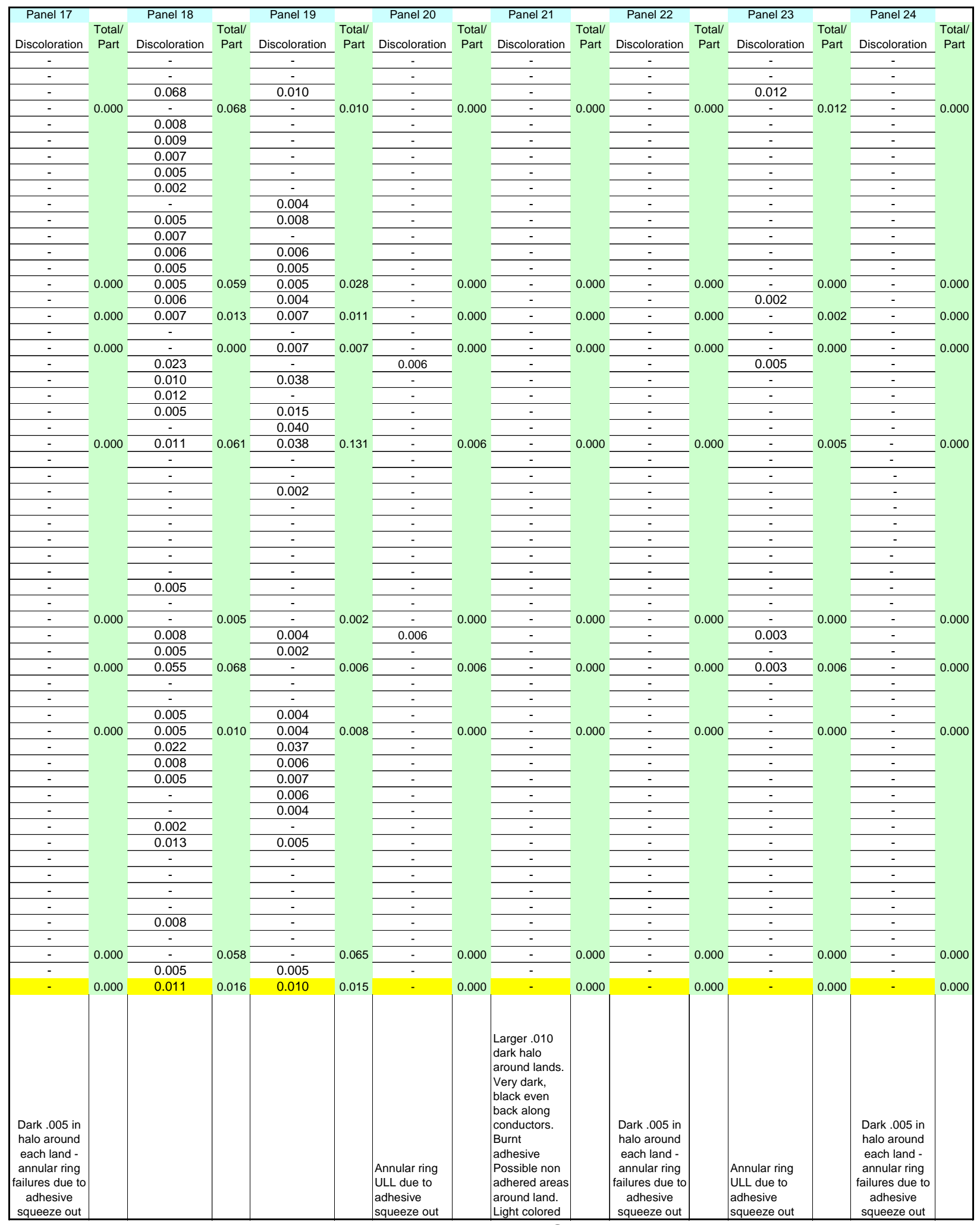

Table 16. Panel 17-24 Delamination/Solder Wicking Results 


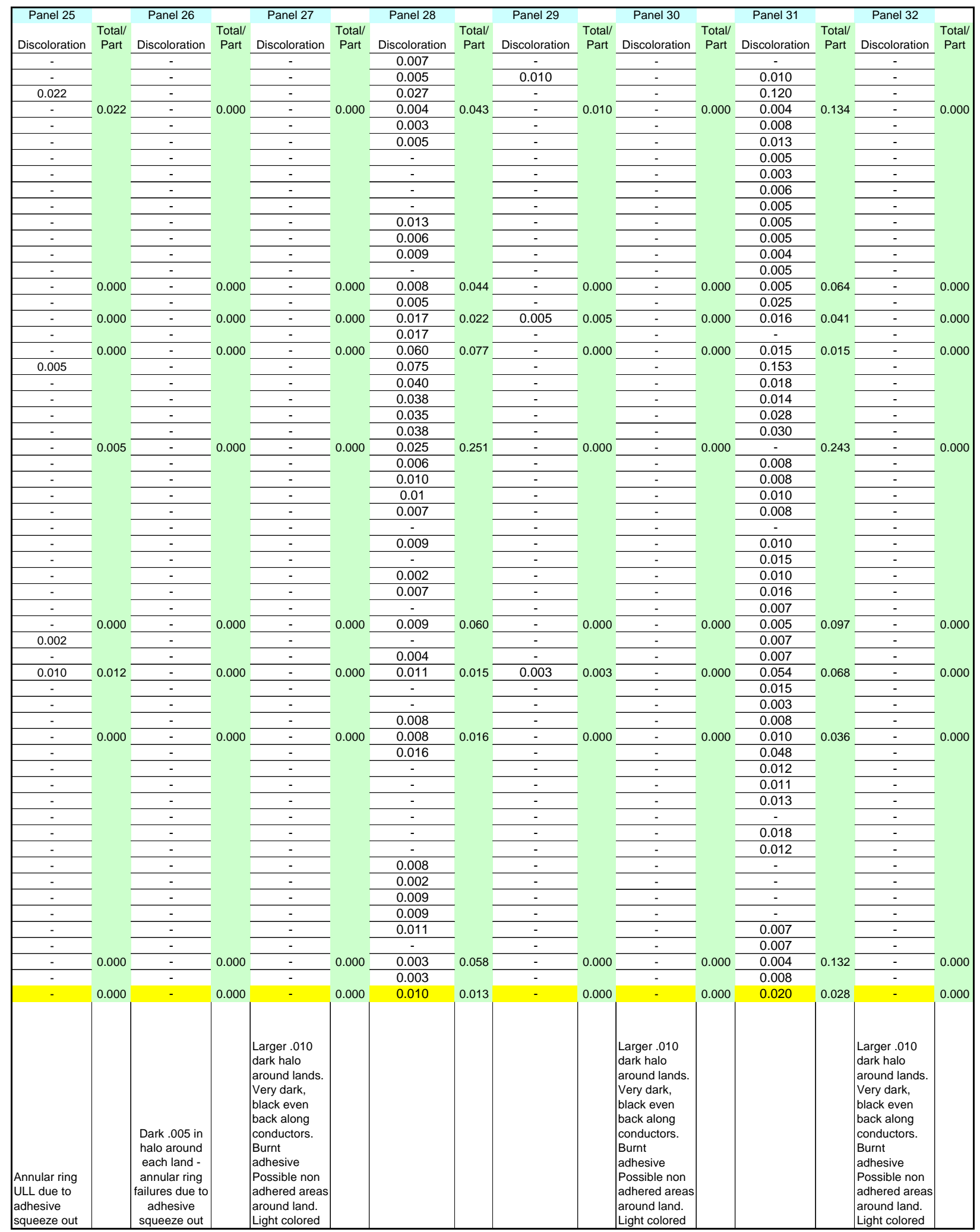

Table 17. Panel 25-32 Delamination/Solder Wicking Results 


\begin{tabular}{|c|c|c|c|c|c|c|c|c|c|c|c|c|c|c|c|c|c|c|c|c|c|}
\hline Panel & & & Total & Rank & & & Total & Rank & & & Total & Rank & & & Total & Rank & & & Total & Rank & \\
\hline 1 & 0.000 & 0.000 & 0.000 & 0 & 0.000 & 0.000 & 0.000 & 0 & 0.000 & 0.000 & 0.000 & 0 & 0.000 & 0.000 & 0.000 & 0 & 0.000 & 0.000 & 0.000 & 0 & 0 \\
\hline 5 & 0.000 & 0.000 & 0.000 & 0 & 0.000 & 0.000 & 0.000 & 0 & 0.000 & 0.000 & 0.000 & 0 & 0.000 & 0.000 & 0.000 & 0 & 0.000 & 0.000 & 0.000 & 0 & 0 \\
\hline 6 & 0.000 & 0.000 & 0.000 & 0 & 0.000 & 0.000 & 0.000 & 0 & 0.000 & 0.000 & 0.000 & 0 & 0.000 & 0.000 & 0.000 & 0 & 0.000 & 0.000 & 0.000 & 0 & 0 \\
\hline 8 & 0.000 & 0.000 & 0.000 & 0 & 0.000 & 0.000 & 0.000 & 0 & 0.000 & 0.000 & 0.000 & 0 & 0.000 & 0.000 & 0.000 & 0 & 0.000 & 0.000 & 0.000 & 0 & 0 \\
\hline 10 & 0.000 & 0.000 & 0.000 & 0 & 0.000 & 0.000 & 0.000 & 0 & 0.000 & 0.000 & 0.000 & 0 & 0.000 & 0.000 & 0.000 & 0 & 0.000 & 0.000 & 0.000 & 0 & 0 \\
\hline 11 & 0.000 & 0.000 & 0.000 & 0 & 0.000 & 0.000 & 0.000 & 0 & 0.000 & 0.000 & 0.000 & 0 & 0.000 & 0.000 & 0.000 & 0 & 0.000 & 0.000 & 0.000 & 0 & 0 \\
\hline 14 & 0.000 & 0.000 & 0.000 & 0 & 0.000 & 0.000 & 0.000 & 0 & 0.000 & 0.000 & 0.000 & 0 & 0.000 & 0.000 & 0.000 & 0 & 0.000 & 0.000 & 0.000 & 0 & 0 \\
\hline 16 & 0.000 & 0.000 & 0.000 & 0 & 0.000 & 0.000 & 0.000 & 0 & 0.000 & 0.000 & 0.000 & 0 & 0.000 & 0.000 & 0.000 & 0 & 0.000 & 0.000 & 0.000 & 0 & 0 \\
\hline 17 & 0.000 & 0.000 & 0.000 & 0 & 0.000 & 0.000 & 0.000 & 0 & 0.000 & 0.000 & 0.000 & 0 & 0.000 & 0.000 & 0.000 & 0 & 0.000 & 0.000 & 0.000 & 0 & 0 \\
\hline 21 & 0.000 & 0.000 & 0.000 & 0 & 0.000 & 0.000 & 0.000 & 0 & 0.000 & 0.000 & 0.000 & 0 & 0.000 & 0.000 & 0.000 & 0 & 0.000 & 0.000 & 0.000 & 0 & 0 \\
\hline 22 & 0.000 & 0.000 & 0.000 & 0 & 0.000 & 0.000 & 0.000 & 0 & 0.000 & 0.000 & 0.000 & 0 & 0.000 & 0.000 & 0.000 & 0 & 0.000 & 0.000 & 0.000 & 0 & 0 \\
\hline 24 & 0.000 & 0.000 & 0.000 & 0 & 0.000 & 0.000 & 0.000 & 0 & 0.000 & 0.000 & 0.000 & 0 & 0.000 & 0.000 & 0.000 & 0 & 0.000 & 0.000 & 0.000 & 0 & 0 \\
\hline 26 & 0.000 & 0.000 & 0.000 & 0 & 0.000 & 0.000 & 0.000 & 0 & 0.000 & 0.000 & 0.000 & 0 & 0.000 & 0.000 & 0.000 & 0 & 0.000 & 0.000 & 0.000 & 0 & 0 \\
\hline 27 & 0.000 & 0.000 & 0.000 & 0 & 0.000 & 0.000 & 0.000 & 0 & 0.000 & 0.000 & 0.000 & 0 & 0.000 & 0.000 & 0.000 & 0 & 0.000 & 0.000 & 0.000 & 0 & 0 \\
\hline 30 & 0.000 & 0.000 & 0.000 & 0 & 0.000 & 0.000 & 0.000 & 0 & 0.000 & 0.000 & 0.000 & 0 & 0.000 & 0.000 & 0.000 & 0 & 0.000 & 0.000 & 0.000 & 0 & 0 \\
\hline 32 & 0.000 & 0.000 & 0.000 & 0 & 0.000 & 0.000 & 0.000 & 0 & 0.000 & 0.000 & 0.000 & 0 & 0.000 & 0.000 & 0.000 & 0 & 0.000 & 0.000 & 0.000 & 0 & 0 \\
\hline 7 & 0.000 & 0.003 & 0.003 & 1 & 0.000 & 0.000 & 0.000 & 0 & 0.000 & 0.000 & 0.000 & 0 & 0.000 & 0.000 & 0.000 & 0 & 0.000 & 0.000 & 0.000 & 0 & 1 \\
\hline 13 & 0.000 & 0.000 & 0.000 & 0 & 0.000 & 0.000 & 0.000 & 0 & 0.000 & 0.000 & 0.000 & 0 & 0.000 & 0.000 & 0.000 & 0 & 0.008 & 0.000 & 0.008 & 1 & 1 \\
\hline 4 & 0.000 & 0.000 & 0.000 & 0 & 0.006 & 0.004 & 0.010 & 1 & 0.000 & 0.000 & 0.000 & 0 & 0.000 & 0.000 & 0.000 & 0 & 0.003 & 0.002 & 0.005 & 1 & 2 \\
\hline 20 & 0.000 & 0.006 & 0.006 & 1 & 0.000 & 0.000 & 0.000 & 0 & 0.000 & 0.000 & 0.000 & 0 & 0.000 & 0.000 & 0.000 & 0 & 0.006 & 0.000 & 0.006 & 1 & 2 \\
\hline 9 & 0.000 & 0.003 & 0.003 & 1 & 0.000 & 0.000 & 0.000 & 0 & 0.003 & 0.000 & 0.003 & 1 & 0.000 & 0.000 & 0.000 & 0 & 0.004 & 0.000 & 0.004 & 1 & 3 \\
\hline 25 & 0.022 & 0.012 & 0.034 & 3 & 0.000 & 0.000 & 0.000 & 0 & 0.000 & 0.000 & 0.000 & 0 & 0.000 & 0.000 & 0.000 & 0 & 0.005 & 0.000 & 0.000 & 0 & 3 \\
\hline 29 & 0.010 & 0.003 & 0.013 & 2 & 0.000 & 0.000 & 0.000 & 0 & 0.005 & 0.000 & 0.005 & 1 & 0.000 & 0.000 & 0.000 & 0 & 0.000 & 0.000 & 0.000 & 0 & 3 \\
\hline 23 & 0.012 & 0.006 & 0.018 & 2 & 0.000 & 0.000 & 0.000 & 0 & 0.002 & 0.000 & 0.002 & 1 & 0.000 & 0.000 & 0.000 & 0 & 0.005 & 0.000 & 0.005 & 1 & 4 \\
\hline 19 & 0.010 & 0.006 & 0.016 & 2 & 0.028 & 0.002 & 0.030 & 3 & 0.011 & 0.015 & 0.026 & 3 & 0.007 & 0.008 & 0.015 & 2 & 0.131 & 0.065 & 0.196 & 10 & 20 \\
\hline 2 & 0.024 & 0.014 & 0.038 & 4 & 0.012 & 0.042 & 0.054 & 6 & 0.008 & 0.014 & 0.022 & 3 & 0.022 & 0.020 & 0.042 & 5 & 0.161 & 0.008 & 0.169 & 10 & 28 \\
\hline 3 & 0.030 & 0.009 & 0.039 & 4 & 0.042 & 0.030 & 0.072 & 8 & 0.012 & 0.020 & 0.032 & 4 & 0.007 & 0.013 & 0.020 & 2 & 0.102 & 0.075 & 0.177 & 10 & 28 \\
\hline 18 & 0.068 & 0.068 & 0.136 & 10 & 0.059 & 0.005 & 0.064 & 7 & 0.013 & 0.016 & 0.029 & 3 & 0.000 & 0.010 & 0.010 & 1 & 0.061 & 0.058 & 0.119 & 10 & 31 \\
\hline 12 & 0.130 & 0.230 & 0.360 & 10 & 0.034 & 0.035 & 0.069 & 7 & 0.016 & 0.016 & 0.032 & 4 & 0.037 & 0.010 & 0.047 & 5 & 0.087 & 0.072 & 0.159 & 10 & 36 \\
\hline 28 & 0.043 & 0.015 & 0.058 & 6 & 0.044 & 0.060 & 0.104 & 10 & 0.022 & 0.013 & 0.035 & 4 & 0.077 & 0.016 & 0.093 & 10 & 0.251 & 0.058 & 0.309 & 10 & 40 \\
\hline 31 & 0.134 & 0.068 & 0.202 & 10 & 0.064 & 0.097 & 0.161 & 10 & 0.041 & 0.028 & 0.069 & 7 & 0.015 & 0.036 & 0.051 & 6 & 0.243 & 0.132 & 0.375 & 10 & 43 \\
\hline 15 & 0.066 & 0.027 & 0.093 & 10 & 0.109 & 0.097 & 0.206 & 10 & 0.035 & 0.013 & 0.048 & 5 & 0.074 & 0.025 & 0.099 & 10 & 0.246 & 0.118 & 0.364 & 10 & 45 \\
\hline
\end{tabular}




\section{Sample (One Ounce Copper Laminate)}

The following five tables contain the actual measured amount of delamination/solder wicking on each land on each panel, soft press pad stackup panels 1 through 36 and hard press pad stackup panels 1 through 9.

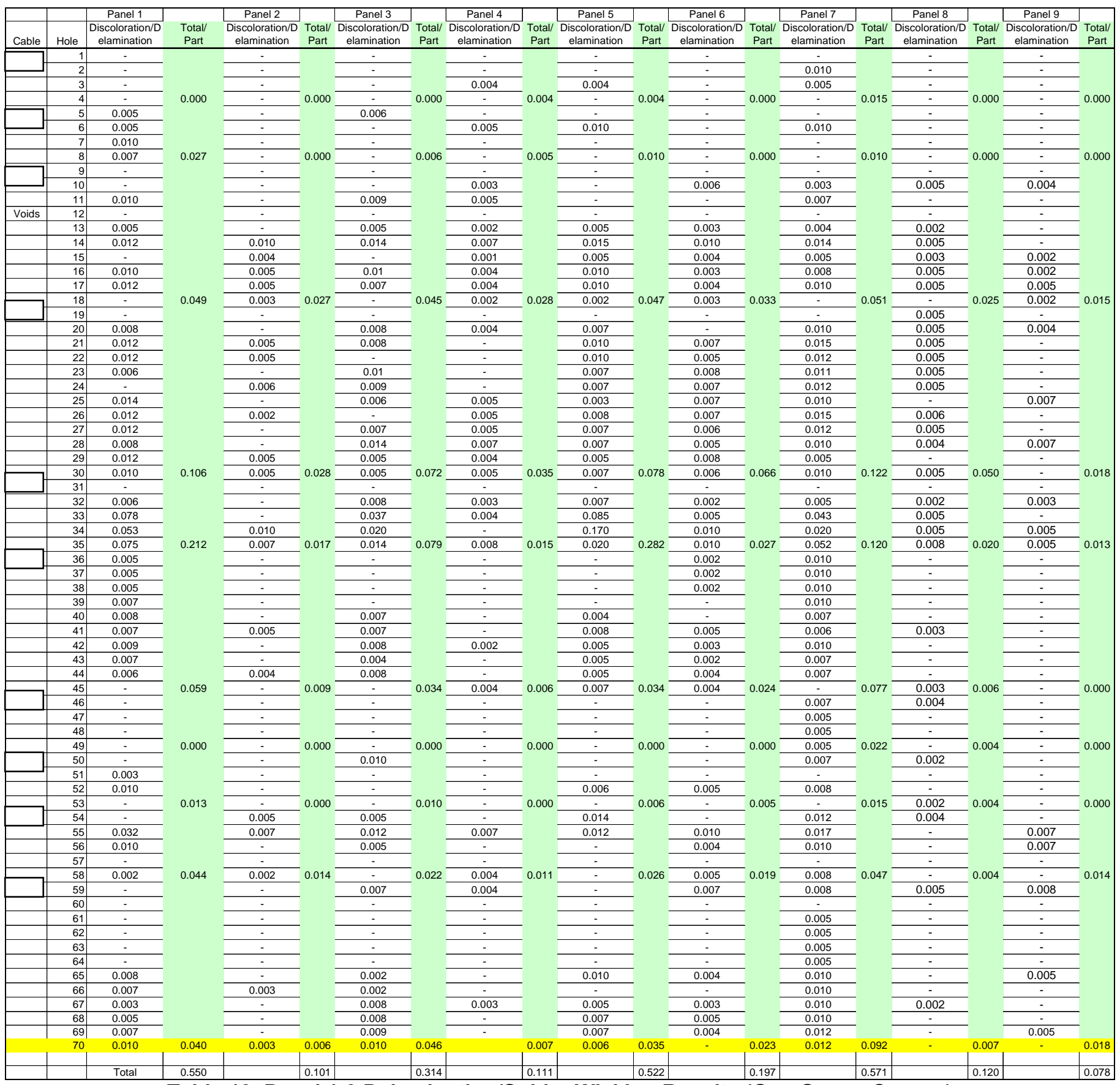

Table 19. Panel 1-9 Delamination/Solder Wicking Results (One Ounce Copper) 


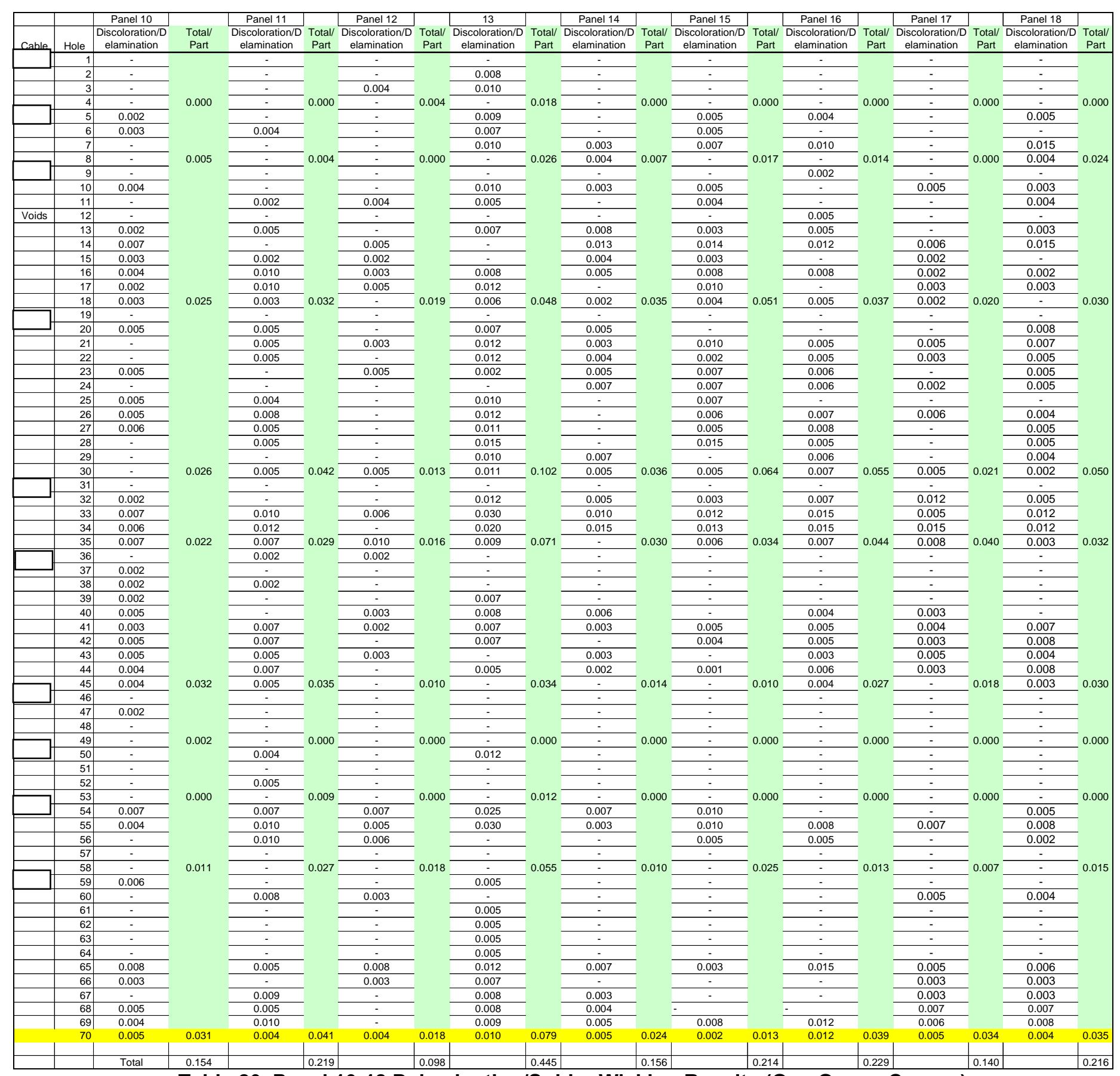

Table 20. Panel 10-18 Delamination/Solder Wicking Results (One Ounce Copper) 


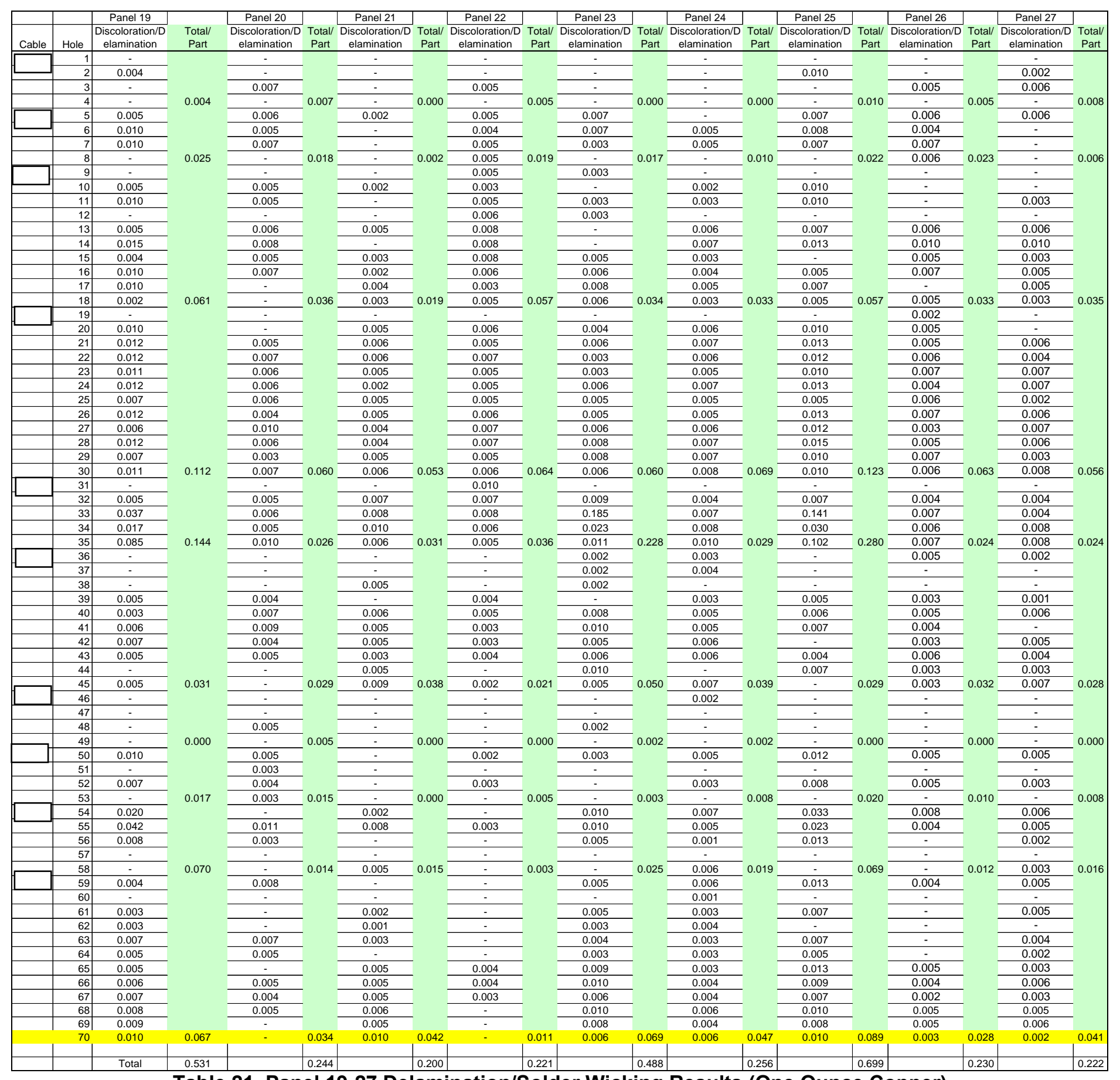

Table 21. Panel 19-27 Delamination/Solder Wicking Results (One Ounce Copper) 


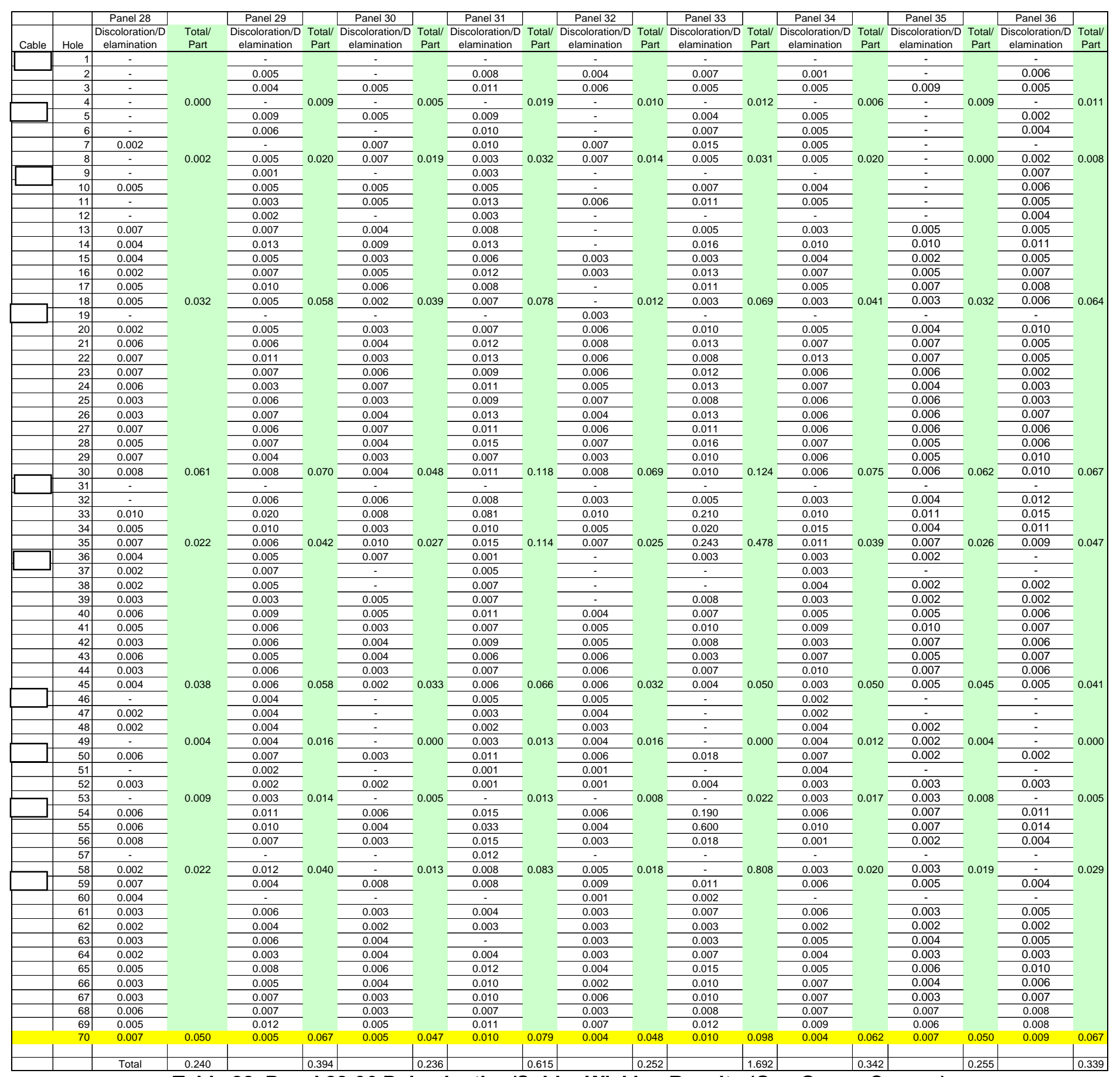

Table 22. Panel 28-36 Delamination/Solder Wicking Results (One Ounce Copper) 


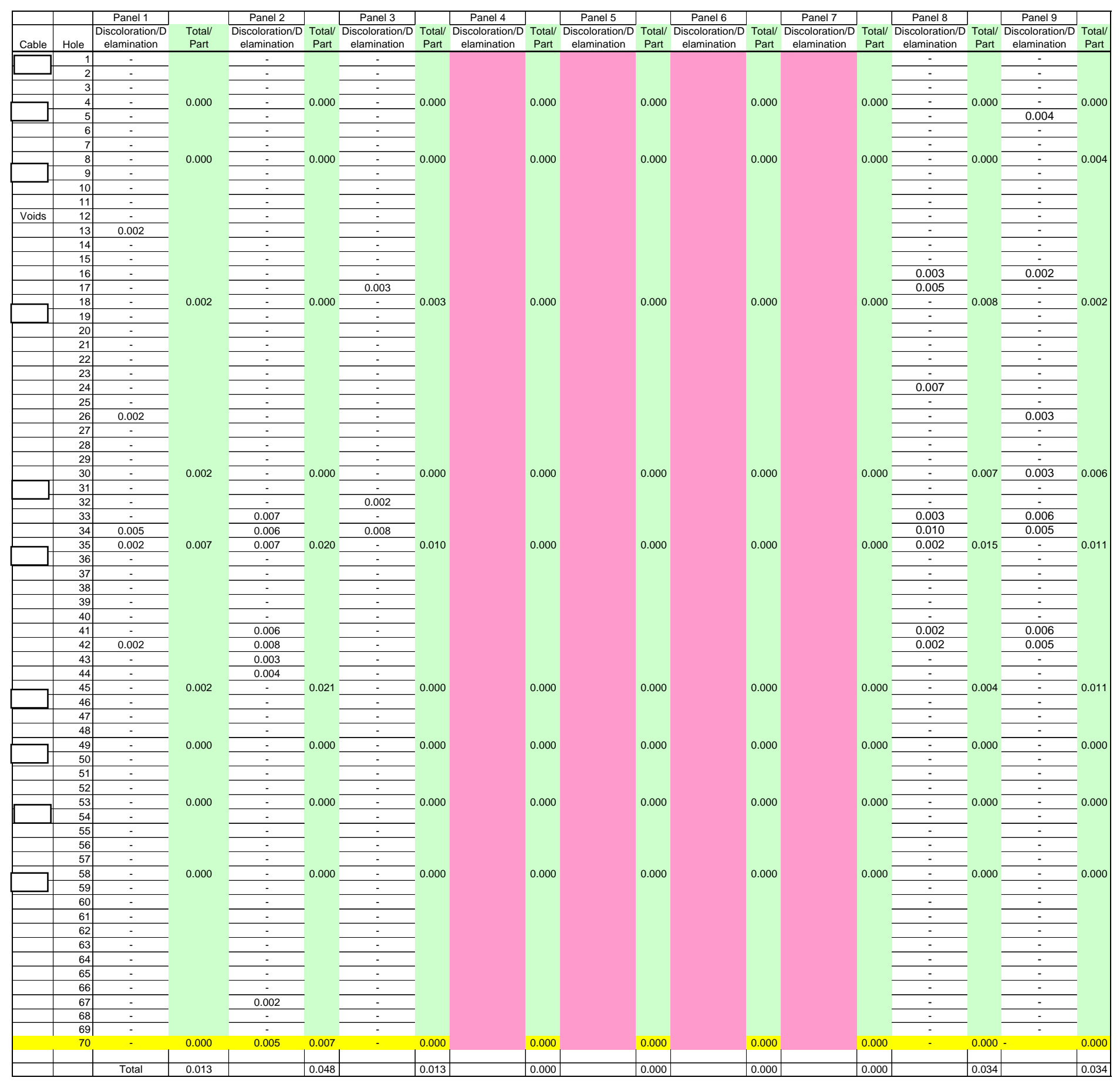

Table 23. Panel Rankings 


\title{
Appendix D \\ Manufacturing Instructions
}

Engineer:

\author{
2 oz. Copper Panels
}

Customer: HPPS/SPPS Experiment

Charge Number:

Due Date: 8/10/07

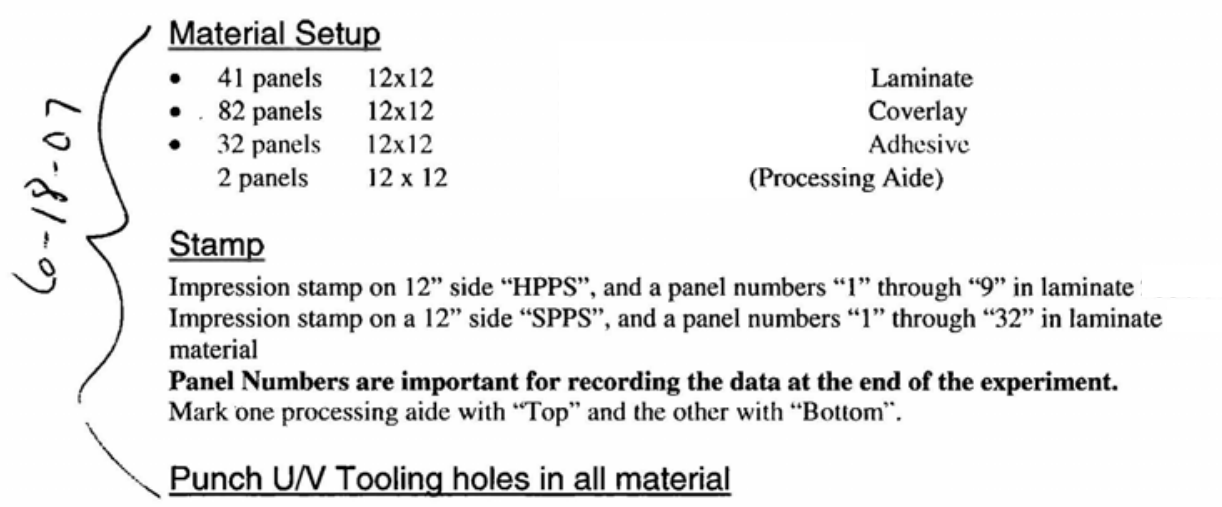

material

Processing Aides

Butt against tooling pins and drill U/V (X0.Y-.625) \& (X11Y-.625) tooling holes in Processing aide material on the marked side. 'Ream as necessary.

Setup of for drilling - Processing Aides

- $12 \times 12$ panels. Normal Stackup.

- Load material on U/V - bottom piece/marking down, top piece/marking up Run program

Setup of for drilling - Laminate

- $12 \times 12$ panels. Normal Stackup.

- Load material on U/V - Stamp side UP Run program

\section{Setup of} for drilling - Top/Bottom Coverlay and Adhesive

- $12 \times 12$ panels. Normal Stackup. TOP COVERLAY - LABEL 41 PIECES OF -952 MATERIAL

- Load material on U/V - release sheet DOWN Run program

ADHESIVE - LABEL 16 PIECES OF -044 MATERIAL

- Load material on U/V - release sheet DOWN Run program

BOTTOM COVERLAY - LABEL 41 PIECES OF -952 MATERIAL

- Load material on U/V - release sheet $\underline{\mathrm{UP}}$ Run program

ADHESIVE - LABEL 16 PIECES OF -044 MATERIAL

- Load material on U/V - release sheet UP Run program

FLEX CABLE 


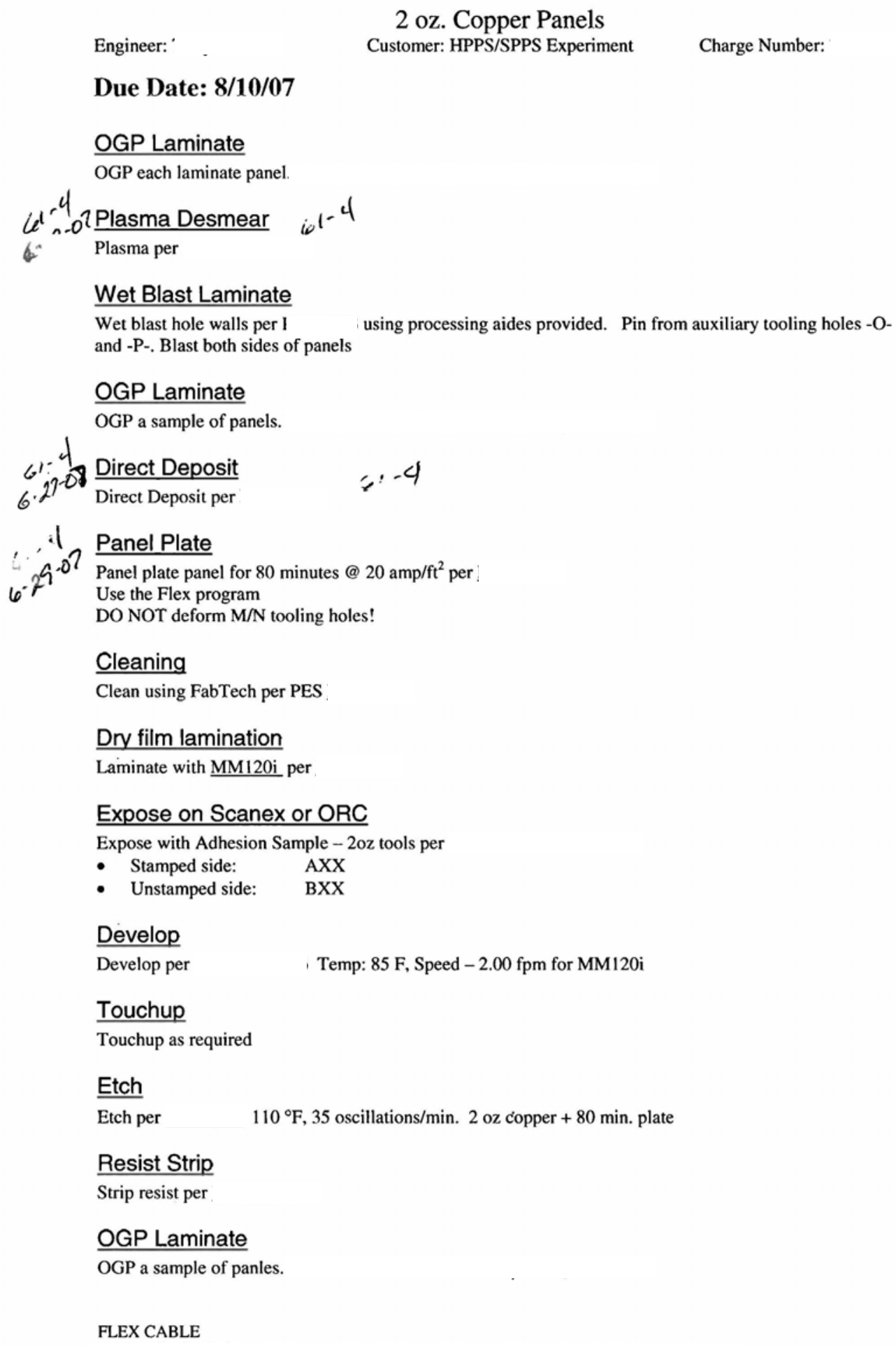

Develop

Develop per Temp: 85 F, Speed -2.00 fpm for MM120i

Touchup

Touchup as required

Etch

Etch per $\quad 110^{\circ} \mathrm{F}, 35$ oscillations $/ \mathrm{min} .2 \mathrm{oz}$ copper $+80 \mathrm{~min}$. plate

Resist Strip

Strip resist per

\section{OGP Laminate}

OGP a sample of panles.

FLEX CABLE 


\section{2 oz. Copper Panels}

Engineer:

\section{Layup}

- Place laminate with impression stamp DOWN.

- Put tooling pins in $M$ \& $N$ to help with registration.

- Place Bottom coverlay on back side of panel. This is marked UP; taking the release sheet off will take the marking off.

- Add the 2 mil Adhesive to half the SPPS panels.

- Heat Tack just the corners and take out the tooling pins.

- Place panel with impression stamp UP

- Place tooling pins in $M$ \& $N$ to help with registration.

- Place Top coverlay on top side of panel. This is marked DOWN

- Add the 2 mil Adhesive to half the SPPS panels.

- Heat Tack just the corners 
Engineer:

2 oz. Copper Panels

Due Date: 8/10/07

\section{Do not process any parts for this page, unless} are present!

\section{Laminate}

1. Plate / Rubber / Teflon / Part / Teflon / Rubber / Plate
a. Panel numbers $-1,2,3$ at 190 ton
b. Panel numbers $-4,5,6$ a 140 ton $13970 \mathrm{n}$
c. Panel numbers $-7,8,9$ at 90 ton

2. Plate/ Paco Pad / Pacothane / Part / Pacothane'/ Paco Pad / Plate

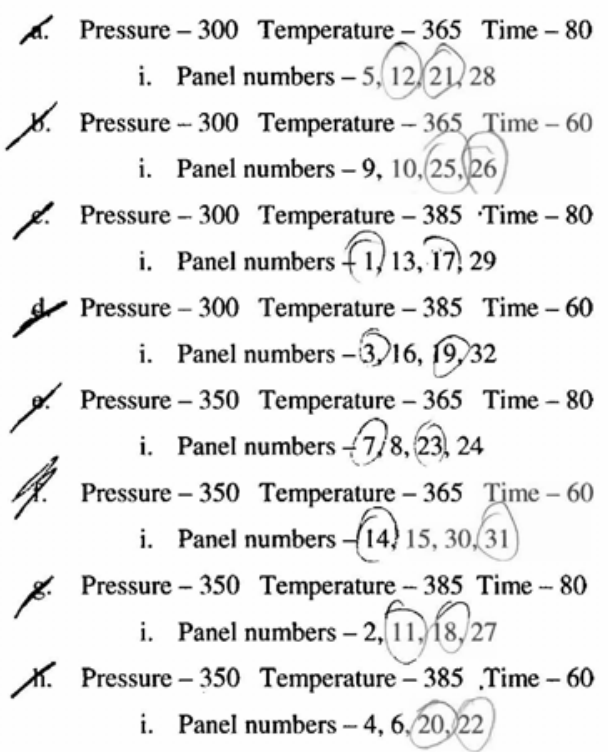

Please record which press was used and the location of the panels in the presses on the following: sheet.

FLEX CABLE 
2 oz. Copper Panels

Customer: HPPS/SPPS Experiment
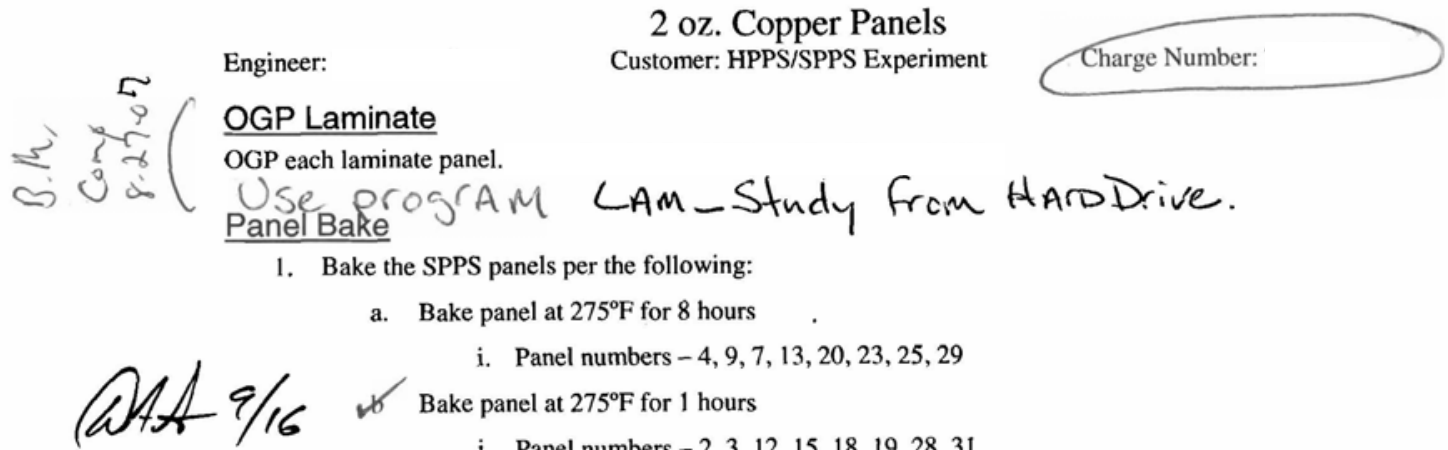

OGP each laminate panel.

Panel Bake

1. Bake the SPPS panels per the following:

a. Bake panel at $275^{\circ} \mathrm{F}$ for 8 hours

i. Panel numbers $-4,9,7,13,20,23,25,29$

Bake panel at $275^{\circ} \mathrm{F}$ for 1 hours

i. Panel numbers $-2,3,12,15,18,19,28,31$

c. Bake panel at $350^{\circ} \mathrm{F}$ for 8 hours

i. Panel numbers - 5, 11, 14, 16, 21, 27, 30, 32

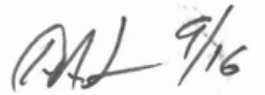

A. Bake panel at $350^{\circ} \mathrm{F}$ for 1 hours

i. Panel numbers $-1,6,8,10,17,22,24,26$

2. Bake the HPPS panels per the following:

a. Bake All panels at $275^{\circ} \mathrm{F}$ for 8 hours

\section{Remove Panel from Oven}

- Remove panels from oven, panels will be hot so use appropriate PPE

\section{Hot Air Solder}

1. Use PES :

2. Record time from oven/lamination to out of post-cleaner on last sheet.

\section{Evaluate Parts}

FLEX CABLE 


\section{2 oz. Copper Panels}

Engineer:

Charge Number:

Hard Press Pad Panels

\begin{tabular}{|c|l|c|}
\hline Panel Number & Press/Date & Location \\
\hline 1 & $T M P-7 / 17 / 07$ & $D$ \\
\hline 2 & $T M P-7 / 7 / 07$ & $E$ \\
\hline 3 & $T m P-7 / 17 / 07$ & $F$ \\
\hline 4 & $T m P-7 / 17 / 07$ & $D$ \\
\hline 5 & $T m P-7 / 17 / 07$ & $E$ \\
\hline 6 & $T M P-7 / 47 / 07$ & F \\
\hline 7 & $T M P-7 / 17 / 07$ & $D$ \\
\hline 8 & $T M P-7 / 17 / 07$ & $E$ \\
\hline 9 & $T M P-7 / 17 / 07$ & F \\
\hline
\end{tabular}

As you are looking at the Presses, all impression stamps towards clean-benches.

TMP

TOP (going left to right)

back of piess

A $B$ C

BOTTOM (going left to right)

for SPPS

D E F

I loaded the stackups

uyself, Lat

FLEX CABLE 
Soft Press Pad Panels

\begin{tabular}{|c|c|c|}
\hline Panel Number & Press/Date $12 ? / 07$ & Location \\
\hline 1 & Tmp & 0 \\
\hline 2 & $\operatorname{Tm} \rho / 8 / 2 / 07$ & A \\
\hline 3 & $\operatorname{Tmp} 712700$ & $D$ \\
\hline 4 & $\operatorname{tin} 7 / 3 / 07$ & \\
\hline 5 & $\operatorname{Tmp} 7 / 26107$ & \\
\hline 6 & $\operatorname{tmp} 8 / 3 / 07$ & 1 \\
\hline 7 & $\operatorname{Tm} 7 / 31 / 07$ & $\cap$ \\
\hline 8 & $\operatorname{tmp} 2 / 31 / 107$ & A \\
\hline 9 & $\operatorname{Tmp} 5 / 26 / 07$ & $D$ \\
\hline 10 & $\operatorname{Tmp}^{2} 3 / 20 / 0$ & $F$ \\
\hline 11 & $\operatorname{Tmp} 8 / 2 ; 07$ & $D$ \\
\hline 12 & Tmp ?L. & $\theta A$ \\
\hline 13 & $\operatorname{Imp} 31$ & $A$ \\
\hline 14 & $T M P$ & $D$ \\
\hline 15 & $7 m \rho$ & A \\
\hline 16 & $T m p$ & $A$ \\
\hline 17 & $\operatorname{Tin} 07 / 27 / 07$ & \\
\hline 18 & $\operatorname{TmP} 8 / 2 / 0 ?$ & $18 F$ \\
\hline 19 & $T M P$ & $F$ \\
\hline 20 & $T m p$ & $A$ \\
\hline 21 & TMP ?/26/07 & $\mathrm{C}$ \\
\hline 22 & TmP 8 & C \\
\hline 23 & $T M P$ & F \\
\hline 24 & Tup $2 / 31100$ & C \\
\hline 25 & $\operatorname{Tmp} 7 / 26 / 07$ & $A$ \\
\hline 26 & tMP $7 / 26 / 07$ & $C$ \\
\hline 27 & Tum $8 / 2 / 07$ & C \\
\hline 28 & $T M P) / g 6 / G$ & F \\
\hline 29 & $\operatorname{Tm} 7 / 23100$ & $C$ \\
\hline 30 & $\operatorname{Tm} 8 / 107$ & C \\
\hline 31 & TMP $8 / 1 / 07$ & $F$ \\
\hline 32 & $\operatorname{tmp} 7 / 27 / 07$ & C \\
\hline
\end{tabular}

FLEX CABLE 
2 oz. Copper Panels

Engineer:
Charge Number:

As you are looking at the Presses, all impression stamps towards clean benches.

TMP

$\overline{\text { TOP }}$ (going left to right)

A B C

BOTTOM (going left to right)

D E F

FLEX CABLE 


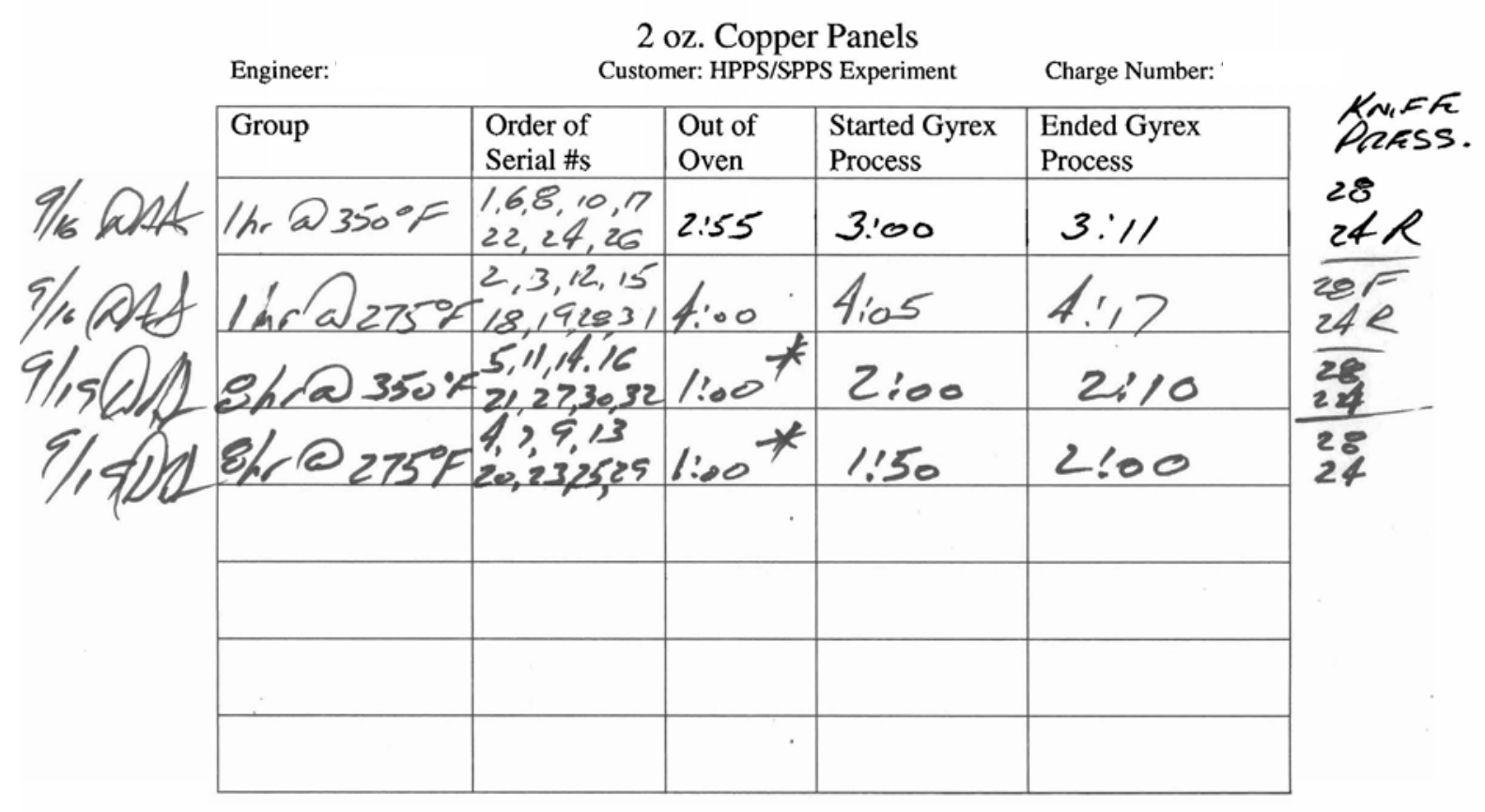

Aur laweus Rued Stamp side Frowt/iop.

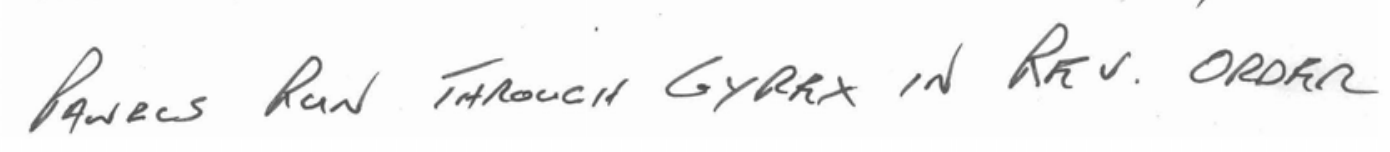
* Storid in $N_{2}$ cabinft from líofm To GyRRX OParation

FLEX CABLE

76 
Engineer:

\section{Due Date: 8/10/07}

\section{Material Setup}

- 41 panels $12 \times 12$

- 82 panels $12 \times 12$

- 32 panels $12 \times 12$

- 2 panels $12 \times 12$
1 oz. Copper Panels

Customer: HPPS/SPPS Experiment
Charge Number:

\section{$\underline{\text { Stamp }}$}

Impression stamp on 12" side "HPPS", and a panel numbers "1" through "9" in laminate material Impression stamp on a 12" side "SPPS", and a panel numbers " 1 " through "32" in laminate material

Panel Numbers are important for recording the data at the end of the experiment.

Mark one processing aide with "Top" and the other with "Bottom".

\section{Punch U/V Tooling holes in all material}

\section{Processing Aides}

Butt against tooling pins and drill U/V (X0.Y-.625) \& (X11Y-.625) tooling holes in

- Processing aide material on the marked side. Ream as necessary.

\section{Setup of for drilling - Processing Aides}

- 12x12 panels. Normal Stackup.

- Load material on U/V - bottom piece/marking down, top piece/marking up Run program

\section{Setup of for drilling - Laminate}

- 12x12 panels. Normal Stackup.

- Load material on U/V - Stamp side UP

Run program

\section{Setup of for drilling - Top/Bottom Coverlay and Adhesive}

- 12x12 panels. Normal Stackup. TOP COVERLAY - LABEL 41 PIECES OF -922 MATERIAL

- Load material on U/V - release sheet DOWN

Run program

- ADHESIVE - LABEL 16 PIECES OF -044 MATERIAL

- Load material on U/V - release sheet DOWN

Run program

BOTTOM COVERLAY - LABEL 41 PIECES OF -922 MATERIAL

- Load material on U/V - release sheet $\underline{\mathrm{UP}}$

Run program

ADHESIVE - LABEL 16 PIECES OF -044 MATERIAL

- Load material on U/V - release sheet UP

Run program 


\section{Due Date: 8/10/07}

\section{OGP Laminate}

OGP each laminate panel.

Use program from hard drive

\section{Plasma Desmear}

Plasma per PES

\section{Wet Blast Laminate}

Wet blast hole walls per using processing aides provided. Pin from auxiliary tooling holes -O- and -P-. Blast both sides of panels

\section{OGP Laminate}

OGP a sample of panels.

Direct Deposit $\quad$ Completed 2-11-2008

Direct Deposit per

\section{Panel Plate Completed 2-19-2008}

Panel plate panel for 80 minutes @ 20 amp/ft ${ }^{2}$ per

Use the Flex program

DO NOT deform $\mathrm{M} / \mathrm{N}$ tooling holes!

\section{Cleaning Completed 3-17-2008}

Clean using FabTech per PES

Dry film lamination Completed 3-17-2008

Laminate with MM120i per

\section{Expose on Scanex or ORC Completed 3-18-2008}

Expose with Adhesion Sample - 1oz tools per

- Stamped side: AXX

- Unstamped side: BXX

Develop Completed 3-18-2008 (61-4)

Develop per PES Temp: 85 F, Speed - 2.00 fpm for MM120i

Touchup Completed 3-18-2008

Touchup as required

Etch Completed 3-20-2008 (61-4)

Etch per, $110^{\circ} \mathrm{F}, 35$ oscillations/min. 1 oz copper +80 min. plate

\section{Resist Strip}

Strip resist per

OGP Laminate Completed 4-18-2008

OGP a sample of panels. 


\section{Layup}

- $\quad$ Place laminate with impression stamp DOWN.

- $\quad$ Put tooling pins in M \& N to help with registration.

- Place Bottom coverlay on back side of panel. This is marked UP; taking the release sheet off will take the marking off.

- Heat Tack just the corners and take out the tooling pins.

- Place panel with impression stamp UP

- Place tooling pins in M \& N to help with registration.

- Place Top coverlay on top side of panel. This is marked DOWN.

- Heat Tack just the corners 
Due Date: 8/10/07

Do not process any parts for this page, unless are present!

\section{Laminate}

1. Plate / Rubber / Teflon / Part / Teflon / Rubber / Plate
a. Panel numbers $-1,2,3$ at 190 ton
b. Panel numbers $4,5,6$ at 140 ton
c. Panel numbers - 7, 8, 9 at 100 ton (227psi)

2. Plate/ Paco Pad / Pacothane / Part / Pacothane / Paco Pad / Plate

a. Pressure $-350 \mathrm{psi}$ Temperature $-385 \mathrm{~F}$ Time-60min

All SPPS panels

Please record which press was used and the location of the panels in the presses on the following sheet.

OGP Laminate Completed 5-1-2008

OGP each laminate panel.

Use program from hard drive

\section{Panel Bake}

1. Bake the SPPS panels per the following:

a. Bake panel at $300^{\circ} \mathrm{F}$ for 1 hour

i. Panel numbers - 1, 7, 13, 19, 25, 31 (Trace Labs Group 1)

b. Bake panel at $300^{\circ} \mathrm{F}$ for 2 hours

i. Panel numbers - 3, 11, 18, 21, 29, 36(HPPS4) (Trace Labs Group 2)

c. Bake panel at $300^{\circ} \mathrm{F}$ for 4 hours

i. Panel numbers - 2, 9, 14, 20, 27, 32 (Trace Labs Group 3)

d. Bake panel at $325^{\circ} \mathrm{F}$ for 1 hour

i. Panel numbers - 5, 15, 16, 23, 33(HPPS5), 34(HPPS7) (Trace Labs Group 4)

e. Bake panel at $325^{\circ} \mathrm{F}$ for 2 hours

i. Panel numbers - 6, 10, 17, 24, 28, 35(HPPS6) (Trace Labs Group 5)

f. Bake panel at $325^{\circ} \mathrm{F}$ for 4 hours

i. Panel numbers - 4, 8, 12, 22, 26, 30 (Trace Labs Group 6)

2. Bake the HPPS panels per the following:

a. Bake ONLY HPPS1, HPPS2, HPPS3, HPPS8, and HPPS9 at $275^{\circ} \mathrm{F}$ for 8 hours (Trace Labs Group 7)

\section{Remove Panel from Oven}

- Remove panels from oven, panels will be hot so use appropriate PPE

\section{$\underline{\text { Hot Air Solder }}$}

1. Use PES. 
Z. Record time from oven/lamination to out of post-cleaner on last sheet.

\section{Evaluate Parts}

Hard Press Pad Panels

\begin{tabular}{|c|c|c|}
\hline Panel Number & Press/Date & Location \\
\hline 1 & TMP 4-28-08 & D \\
\hline 2 & TMP 4-28-08 & E \\
\hline 3 & TMP 4-28-08 & F \\
\hline 4 & & \\
\hline 5 & & \\
\hline 6 & & D \\
\hline 7 & & F \\
\hline 8 & TMP 4-28-08 & \\
\hline 9 & TMP 4-28-08 & \\
\hline
\end{tabular}

As you are looking at the Presses, all impression stamps towards clean benches.

$\underline{T M P}$

TOP (going left to right)

A B C

BOTTOM (going left to right)

D E F 
Soft Press Pad Panels

\begin{tabular}{|c|c|c|}
\hline Panel Number & Press/Date & Location \\
\hline 1 & Wabash 4-25-08 & $\mathrm{A}$ \\
\hline 2 & TMP 4-24-08 & $\mathrm{D}$ \\
\hline 3 & Wabash 4-24-08 & $\mathrm{D}$ \\
\hline 4 & Wabash 4-25-08 & F \\
\hline 5 & TMP 4-24-08 & $\mathrm{E}$ \\
\hline 6 & TMP 4-25-08 & A \\
\hline 7 & Wabash 4-25-08 & $\mathrm{C}$ \\
\hline 8 & Wabash 4-24-08 & A \\
\hline 9 & Wabash 4-24-08 & F \\
\hline 10 & TMP 4-25-08 & $\mathrm{D}$ \\
\hline 11 & Wabash 4-24-08 & $\mathrm{C}$ \\
\hline 12 & Wabash 4-24-08 & $E$ \\
\hline 13 & Wabash 4-24-08 & B \\
\hline 14 & TMP 4-24-08 & $\bar{E}$ \\
\hline 15 & Wabash 4-25-08 & F \\
\hline 16 & Wabash 4-25-08 & $\mathrm{D}$ \\
\hline 17 & Wabash 4-25-08 & $\bar{E}$ \\
\hline 18 & Wabash 4-25-08 & B \\
\hline 19 & TMP 4-25-08 & B \\
\hline 20 & Wabash 4-25-08 & B \\
\hline 21 & TMP 4-24-08 & A \\
\hline 22 & TMP 4-25-08 & $\mathrm{C}$ \\
\hline 23 & TMP 4-24-08 & B \\
\hline 24 & TMP 4-25-08 & A \\
\hline 25 & Wabash 4-25-08 & $\mathrm{C}$ \\
\hline 26 & TMP 4-25-08 & B \\
\hline 27 & Wabash 4-25-08 & $E$ \\
\hline 28 & Wabash 4-25-08 & A \\
\hline 29 & TMP 4-25-08 & F \\
\hline
\end{tabular}




\begin{tabular}{|c|c|c|}
\hline 30 & TMP 4-25-08 & E \\
\hline 31 & TMP 4-25-08 & E \\
\hline 32 & TMP 4-25-08 & F \\
\hline 33 HPPS5 & Wabash 4-25-08 & D \\
\hline 34 HPPS7 & TMP 4-25-08 & C \\
\hline 35 HPPS6 & TMP 4-25-08 & C \\
\hline 36 HPPS4 & TMP 4-24-08 & \\
\hline
\end{tabular}

As you are looking at the Presses, all impression stamps towards clean benches.

TMP

TOP (going left to right)

A B C

BOTTOM (going left to right)

D E F

Tuesday 5/13/08

Wednesday 5/14/08

Wednesday $5 / 14 / 08$
HPPS Panels

2:30 PM Bake all HPPS Panels except for 4, 5, 6, and 7

6:30 AM Gyrex all HPPS Panels except for $4,5,6$, and 7

\section{SPPS Panels}

7:00 AM Panel \#'s: 1, 7, 13, 19, 25, 31

8:00 AM Gyrex Panels: 1, 7, 13, 19, 25, 31

8:00 AM Panel \#'s: 3, 11, 18, 21, 29, HPPS4

10:00 AM Gyrex Panels: 3, 11, 18, 21, 29, HPPS4

10:00 AM Panel \#'s: 2, 9, 14, 20, 27, 32

2:00 PM Gyrex Panels: 2, 9, 14, 20, 27, 32

Thursday 5/15/08

7:00 AM Panel \#'s: 5, 15, 16, 23, HPPS5, HPPS7

8:00 AM Gyrex Panels: 5, 15, 16, 23, HPPS5, HPPS7

8:00 AM Panel \#'s: 6, 10, 17, 24, 28, HPPS6

10:00 AM Gyrex Panels: 6, 10, 17, 24, 28, HPPS6

10:00 AM Panel \#'s: 4, 8, 12, 22, 26, 30

2:00 PM Gyrex Panels: 4, 8, 12, 22, 26, 30
Bake Temperature

275

Bake Temperature

300

300

300

Bake Temperature

325

325

325
Bake Time

8 hours

\section{Bake Time}

1 hour

2 hours

4 hours

Bake Time

1 hour

2 hours

4 hours 


\section{Appendix E}

Trace Laboratories Test Reports

Phase Three Two Ounce Copper Laminate Environmental Test Report ..... 85-111

Phase Three One Ounce Copper Laminate Environmental Test Report.......112-139 


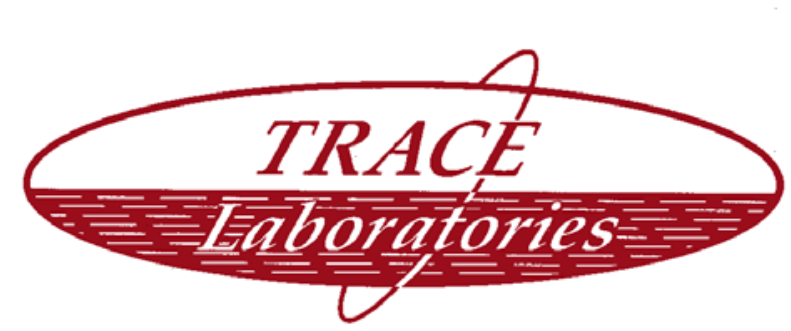

\section{TEST REPORT FOR: \\ HONEYWELL, FM\&T}

\section{DATE IN:}

Attn:

November 8, 2007

P/O \#:

\section{SUBMISSION IDENTIFICATION:}

Four (4) lots of flexible printed circuit boards were submitted for Bending Test, MIR and Thermal Shock in accordance with IPC-6013A, Amendment 2, Class 3. The boards were identified as follows:

\begin{tabular}{|l|l|l|l|}
\hline & & & \\
\hline & & & \\
\hline & & & \\
\hline & & & \\
\hline & & & \\
\hline
\end{tabular}

SUMMARY:

The boards met the requirements of IPC-6013A, Amendment 2, Class 3, for Bending Test, MIR and Thermal Shock

\section{APPROVED:}

SAMPLE DISPOSITION: Samples returned to the customer
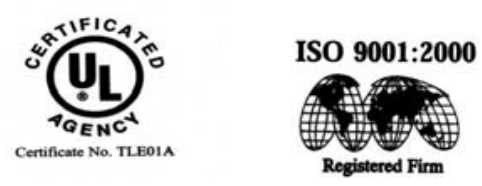


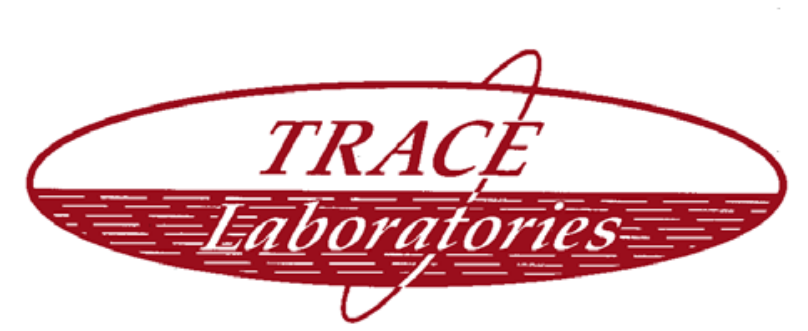

BENDING TEST

\section{TEST SPECIMENS:}

Four (4) lots of flexible printed circuit boards.

\section{REFERENCE:}

IPC-6013A, Amendment 2, Class 3, paragraphs 3.6.1, 3.9 and 3.3.

\section{REQUIREMENT:}

After completion of the bending test in both directions, the flexible or rigid flexible printed wiring board shall be tested for electrical defects in accordance with 3.9 and shall meet the visual requirements of 3.3

\section{METHOD:}

The bending test shall conform to Figure 3-8, unless otherwise agreed to by the user. The bending test requirements shall be as specifies on the appropriate document / drawing. See IPC 2223 for guidance on the minimum bend radii. The following parameters shall be specified as minimum:

- Direction of bend (a).

- Degree of bend (b)

- Number of bend cycles (c)

- Diameter of mandrel (d)

- Points of application to be supplied by user.

A bend cycle is defined as taking one end of the specimen and bending it around a mandrel and then bending back to the original starting point, traveling $180^{\circ}$ in one direction and $180^{\circ}$ in the opposite direction. A bend cycle may also be defined as bending (using opposite ends) the ends towards each other (bend the same direction) and then bending them back to the original starting position, with each end traveling $90^{\circ}$ in the opposite direction.
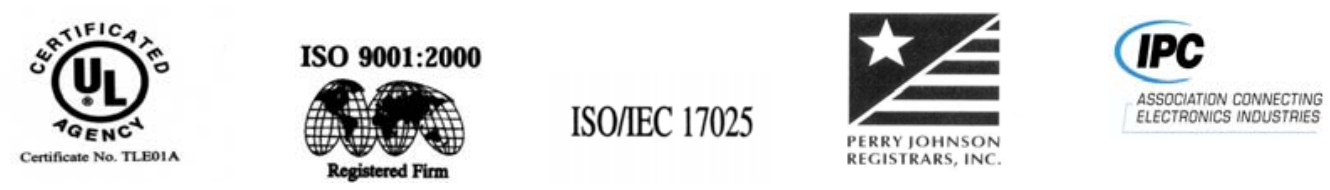

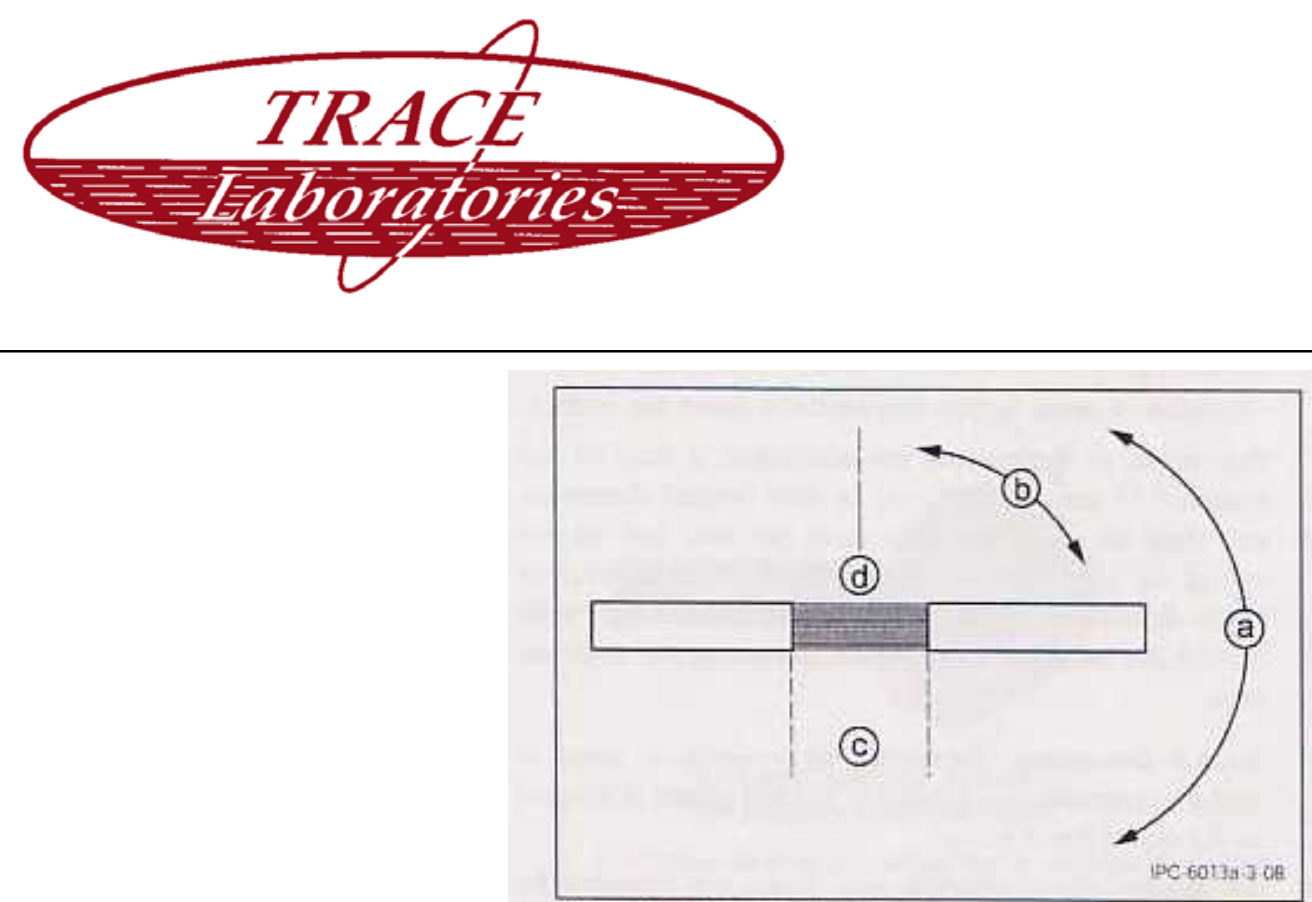

Figure 3-8 Bending Test

The direction of the bend was $90^{\circ}$ upward and $90^{\circ}$ downward.

The degree of the bends was $90^{\circ}$.

The number of bend cycles was 25 .

Mandrel Diameter:

\begin{tabular}{|c|c|c|}
\hline & 10 times the thickness & Mandrel size \\
\hline Part Number & mils & $7 / 32^{\prime \prime}$ \\
\hline & mils & $3 / 16^{\prime \prime}$ \\
\hline & mils & $3 / 16^{\prime \prime}$ \\
\hline & mils & $3 / 16^{\prime \prime}$ \\
\hline
\end{tabular}

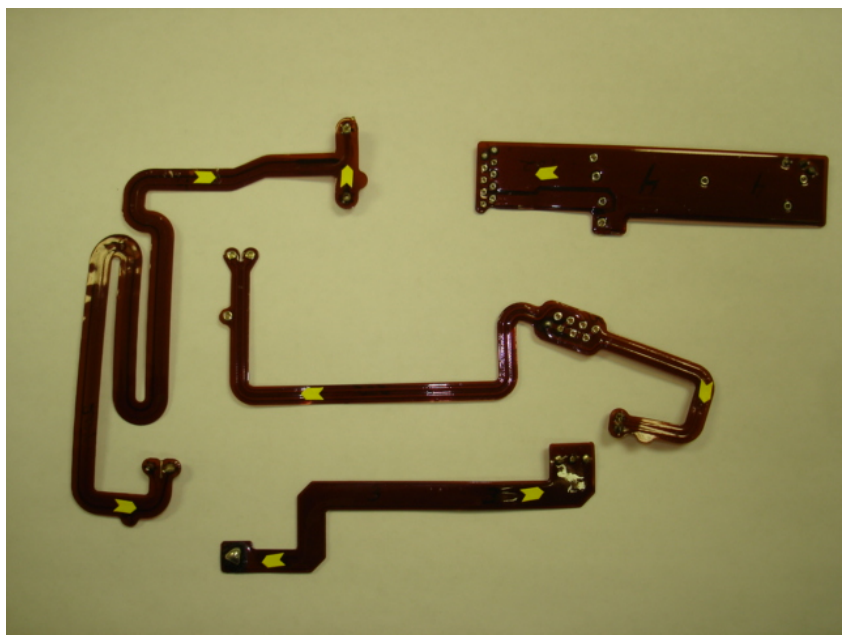

Photo 1: Bend Locations

After completing the bending test the boards were tested per electrical requirements 3.9.
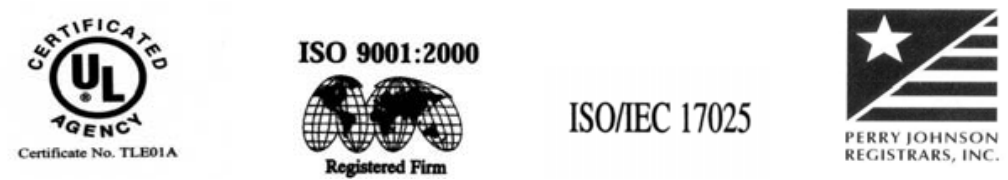


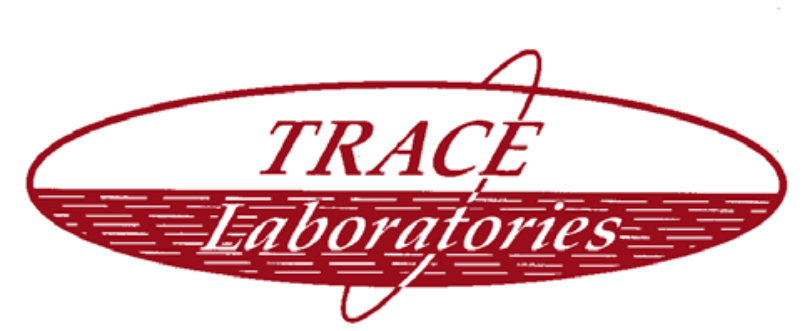

\section{ELECTRICAL REQUIREMENTS}

\section{DIELECTRIC WITHSTANDING VOLTAGE}

\section{TEST SPECIMENS:}

Four (4) lots of flexible printed circuit boards.

\section{REFERENCE:}

IPC-6013A, Amendment 2, Class 3, paragraph 3.9.1

\section{REQUIREMENT:}

Applicable test coupons or production boards shall meet the requirements of Table 3-12, without flashover, sparkover, or breakdown between conductor, or conductors and lands. The dielectric withstanding voltage test shall be performed in accordance with IPC-TM-650, method 2.5.7D. The dielectric withstanding voltage shall be applied between all common portions of each conductor pattern and adjacent common portions of each conductor pattern. The voltage shall be applied between conductor patterns of each layer and the electrically isolated pattern of each adjacent layer.

Table 3-12 Dielectric Withstanding Voltage

\begin{tabular}{|l|c|c|}
\cline { 2 - 3 } \multicolumn{1}{c|}{} & Class 2 & Class 3 \\
\hline Voltage & $500 \mathrm{VDC}$ & $1000 \mathrm{VDC}$ \\
& $+15,-0$ & $+25,-0$ \\
\hline Time 30 & seconds & 30 seconds \\
& $+3,-0$ & $+3,-0$ \\
\hline
\end{tabular}

\section{METHOD:}

Insulated wires were soldered to a set of adjacent parallel conductors. The electrodes of the hi-pot tester were connected to the insulated wires. Five hundred VDC were applied for 30 seconds. The results were recorded.

\section{RESULTS:}

There was no flashover, or breakdown between conductor, or conductors and lands between all common portions of each conductor pattern and adjacent common portions of each conductor pattern.
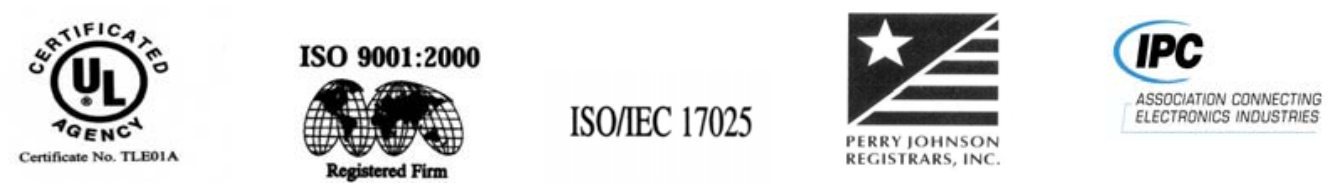


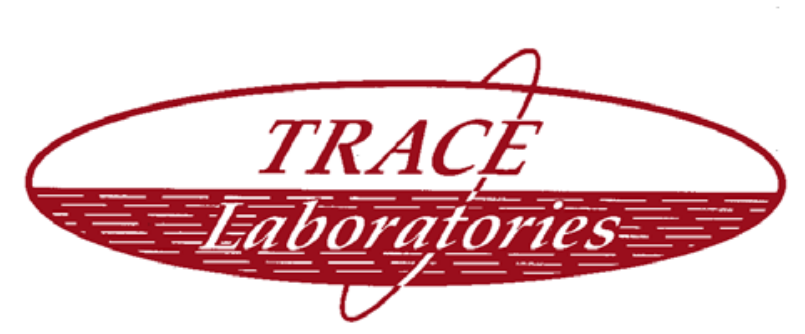

\section{CONTINUITY}

TEST SPECIMENS:

Four (4) lots of flexible printed circuit boards.

\section{REFERENCE:}

IPC-6013A, Amendment 2, Class 3, paragraph 3.9.2.1.

\section{REQUIREMENT:}

Flexible printed boards and qualification testing of flexible printed wiring boards shall be tested in accordance with the procedure outlined below. There shall be no circuits whose resistance exceeds the values established in the procurement documentation. The presence of long runs of very narrow conductors, or high resistance metals may increase these values. When required by the user, interconnect shorts and continuity coupon D shall be used for evaluation of the interconnection resistance and circuit continuity.

A current shall be passed through each conductor or group of interconnected conductors by applying electrodes on the terminals at each end of the conductor or group of conductors. The current passing through the conductors shall not exceed that specified in IPC-2221 for the smallest conductor in the circuit. For qualification, the test current shall not exceed one ampere. Flexible printed wiring with designed resistive patterns shall meet the resistance requirements specified on the procurement documentation.

\section{METHOD:}

Insulated wires were soldered to the plated through holes at the ends of a selected conductor. The wires were placed in the grips of a multimeter. A resistance measurement was observed.

\section{RESULTS:}

The boards met the requirements for continuity between the conductor and land patterns.
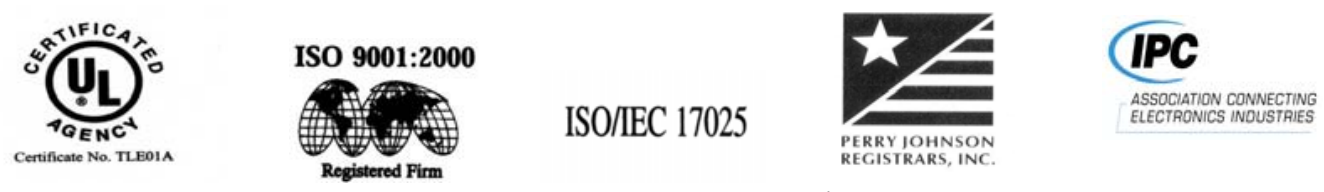


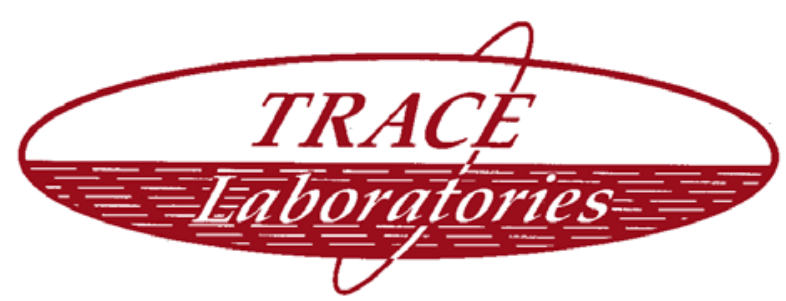

\section{ISOLATION (CIRCUIT SHORTS)}

\section{TEST SPECIMENS:}

Four (4) lots of flexible printed circuit boards.

\section{REFERENCE:}

IPC-6013A, Amendment 2, Class 3, paragraph 3.9.2.2.

\section{REQUIREMENT:}

Flexible printed wiring shall be tested in accordance with the following procedure. The isolation resistance between conductors shall meet the values established in the procurement documentation.

The voltage applied between the networks must be high enough to provide sufficient current resolution for the measurement. At the same time, it must be low enough to prevent arc-over between adjacent networks, which could induce defects within the product. For manual testing, the voltage shall be 200 volts minimum and shall be applied for a minimum of 5 seconds. When automatic test equipment is used, the minimum applied voltage shall be the maximum rated voltage of the flexible printed wiring. When not specified, the default value of 40 volts shall be used

\section{METHOD:}

Insulated wires were soldered to the plated through holes at the ends of a selected conductor. The wires were placed in the grips of a multimeter. The resistance measurement was obtained.

\section{RESULTS:}

The boards met the requirements for isolation (circuit shorts).

Part Number

\begin{tabular}{|l|c|c|l|l|}
\hline & Layer 103-1 & $303-3$ 503-5 803-8 & & \\
\hline Continuity & $1-1$ & Pass Pass Pass Pass & & \\
\hline & $2-2$ & Pass Pass Pass Pass & & \\
\hline & $3-3$ & Pass Pass Pass Pass & & \\
\hline Isolation & $1-1$ & Pass Pass Pass Pass & & \\
\hline & $2-3$ & Pass Pass Pass Pass & & \\
\hline
\end{tabular}
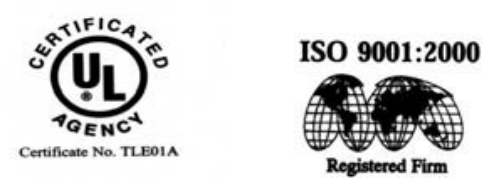


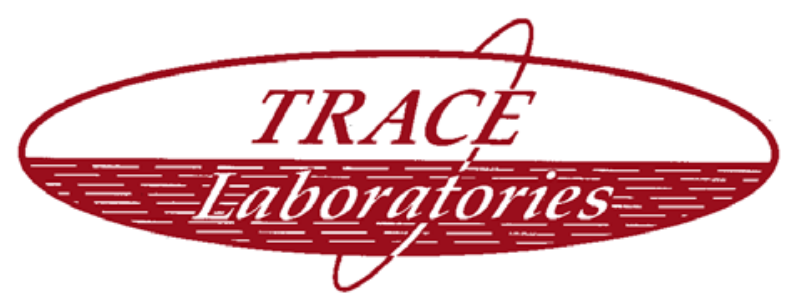

Part Number

\begin{tabular}{|c|c|c|l|l|}
\hline & Layer 202-2 & $402-4$ 602-6 902-9 & & \\
\hline Continuity & $1-1$ & Pass Pass Pass Pass & & \\
\hline & $2-2$ & Pass Pass Pass Pass & & \\
\hline & $3-3$ & Pass Pass Pass Pass & & \\
\hline Isolation & $1-2$ & Pass Pass Pass Pass & & \\
\hline & $2-3$ & Pass Pass Pass Pass & & \\
\hline
\end{tabular}

Part Number

\begin{tabular}{|c|c|c|l|l|}
\hline & Layer 305-3 & $505-5705-7805-8$ & & \\
\hline Continuity & $1-1$ & Pass Pass Pass Pass & & \\
\hline & $2-2$ & Pass Pass Pass Pass & & \\
\hline Isolation & $1-2$ & Pass Pass Pass Pass & & \\
\hline
\end{tabular}

Part Number

\begin{tabular}{|c|c|c|c|c|}
\hline & Layer 204-2 604-6 704-7 & & \\
\hline Continuity 1-1 & Pass & Pass & Pass \\
\hline 2-2 & & Pass & Pass & Pass \\
\hline Isolation 1-2 & & Pass & Pass & Pass \\
\hline
\end{tabular}

\section{MOISTURE AND INSULATION RESISTANCE}

\section{TEST SPECIMENS:}

Four (4) lots of flexible printed circuit boards.

\section{REFERENCE:}

IPC-6013A, Amendment 2, Class 3, paragraph 3.10.1.

\section{REQUIREMENT:}

The flexible wiring boards shall not exhibit measling, blistering, or delamination in excess of that allowed in 3.3.2. Insulation resistance shall meet the requirements shown in table 3-13. Noncomponent flush wiring shall have a minimum requirement of $50 \mathrm{M} \Omega[5.0 \mathrm{E}+9]$ for all classes. Moisture and insulation resistance testing for flexible printed wiring shall be performed in accordance with IPC-TM-650, Method 2.6.3F.
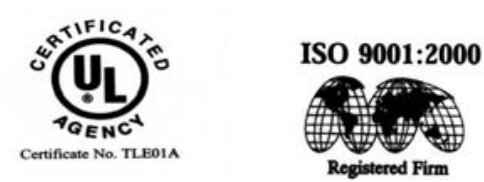

ISO/IEC 17025
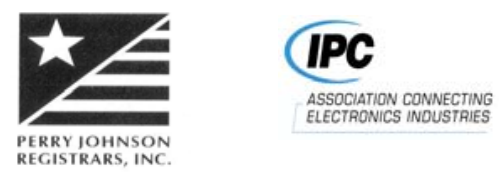


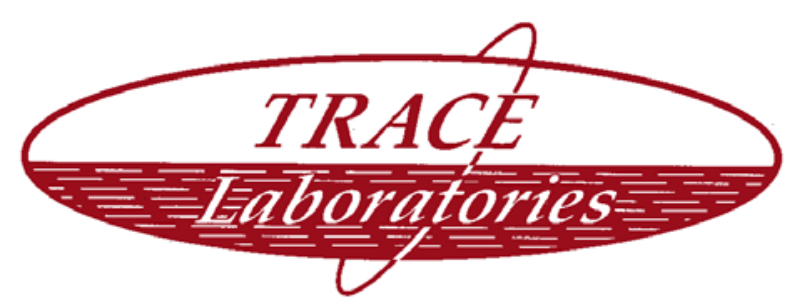

\section{METHOD:}

Insulated single stranded wires were soldered to each of the connection points of the test specimen. These wires were used to connect the test patterns of the test specimen in order to obtain the insulation resistance measurements and to allow a connection with the outside power supply. The test leads were cleaned with isopropyl alcohol and scrubbed with a soft bristle brush for a minimum of 30 seconds. The test specimen was rinsed thoroughly with fresh isopropyl alcohol from top to bottom. The cleaned area was rinsed thoroughly with fresh deionized water holding the test specimen at an approximate $30^{\circ}$ angle and sprayed from top to bottom. The test specimen was dried in an oven for a minimum of three hours at an oven temperature of $50 \pm 5^{\circ} \mathrm{C}\left(122 \pm 9^{\circ} \mathrm{F}\right)$. The test specimen was then coated with conformal coating in accordance with IPC-CC-830 and cured as specified by the coating supplier. After curing the test specimens were then stabilized to ambient temperature. Initial insulation resistance measurements were taken by applying five hundred (500) volts direct current for one minute using a high resistance meter.

The test specimen was placed in the vertical position in a moisture chamber and the wires were connected to a power supply throughout the entire test. A polarizing voltage of 100 volts direct current was applied. The test sample was exposed to 20 cycles of temperature and humidity. The humidity chamber was cycled from $25^{\circ} \mathrm{C},+5^{\circ} \mathrm{C},-2^{\circ} \mathrm{C}$ at $85 \%$ to $93 \%$ relative humidity, to $65^{\circ} \mathrm{C}$, $\pm 2^{\circ} \mathrm{C}$ at $85 \%$ to $93 \%$ relative humidity.

Note: The humidity may drop to a minimum of $80 \%$ relative humidity when going from high to low temperature.

Insulation resistance measurements were made within two hours after removal from the chamber. Following 24 hours stabilization period, the test samples were visually examined.

Table 3-13 Insulation Resistance

\begin{tabular}{|c|c|c|}
\hline & Class 2 & Class 3 \\
\hline As Received & $500 \mathrm{M} \Omega 500$ & $\mathrm{M} \Omega$ \\
\hline After exposure to moisture & $100 \mathrm{M} \Omega 500$ & $\mathrm{M} \Omega$ \\
\hline
\end{tabular}

\section{RESULTS:}

Visual Results:

There was no evidence of mealing of the conformal coating.
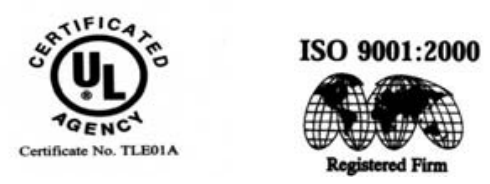

ISO/IEC 17025
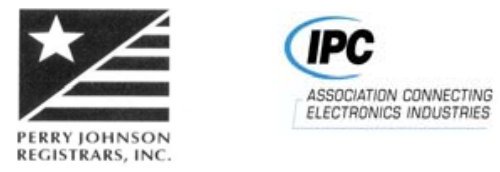


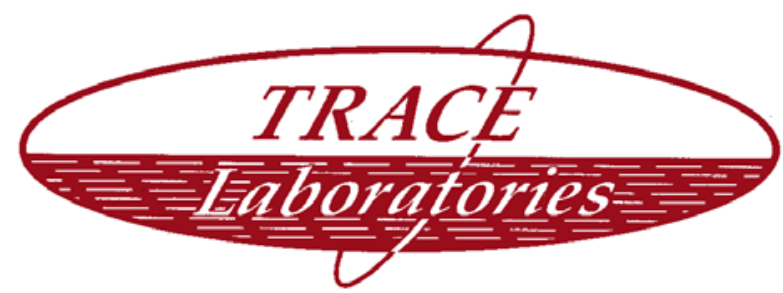

Insulation Resistance Measurements (in megohms):

Part Number

\begin{tabular}{|l|l|c|c|}
\hline S/n & Layer & Before Conditioning & After Conditioning \\
\hline $1031-1$ & & $>10^{7}$ & $1.77 \mathrm{E}+07$ \\
\hline $1031-2$ & & $>10^{7} 5.50 \mathrm{E}+06$ & \\
\hline $3031-1$ & & $>10^{7} 1.74 \mathrm{E}+07$ & \\
\hline $3031-2$ & & $>10^{7} 1.90 \mathrm{E}+07$ & \\
\hline $5031-1$ & & $>10^{7} 1.51 \mathrm{E}+07$ & \\
\hline $5031-2$ & & $>10^{7} 2.71 \mathrm{E}+07$ & \\
\hline $8031-1$ & & $>10^{7} 1.49 \mathrm{E}+07$ & \\
\hline $8031-2$ & & & \\
\hline
\end{tabular}

Part Number

\begin{tabular}{|l|l|r|l|}
\hline S/n & Layer & Before Conditioning & After Conditioning \\
\hline $2021-1$ & & $>10^{7} 1.64 \mathrm{E}+07$ & \\
\hline $2021-2$ & & $>10^{7} 1.24 \mathrm{E}+07$ & \\
\hline $4021-1$ & & $>10^{7} 4.09 \mathrm{E}+07$ & \\
\hline $4021-2$ & & $>10^{7} 2.06 \mathrm{E}+07$ & \\
\hline $6021-1$ & & $>10^{7} 2.69 \mathrm{E}+07$ & \\
\hline $6021-2$ & & $>10^{7} 1.51 \mathrm{E}+07$ & \\
\hline $9021-1$ & & $>10^{7} 3.33 \mathrm{E}+07$ & \\
\hline $9021-2$ & & $>10^{7} 2.74 \mathrm{E}+07$ & \\
\hline
\end{tabular}

Part Number

\begin{tabular}{|l|r|r|r|}
\hline S/n & Layer & Before Conditioning & After Conditioning \\
\hline $3051-1$ & & $>10^{7} 1.41 \mathrm{E}+07$ & \\
\hline $5051-1$ & & $>10^{7} 1.19 \mathrm{E}+07$ & \\
\hline $7051-1$ & & $>10^{7} 1.25 \mathrm{E}+07$ & \\
\hline $8051-1$ & & $>10^{7} 7.75 \mathrm{E}+06$ & \\
\hline
\end{tabular}

Part Number

\begin{tabular}{|c|r|r|r|}
\hline S/n & Layer & Before Conditioning & After Conditioning \\
\hline $2041-1$ & & $>10^{7} 1.34 \mathrm{E}+07$ & \\
\hline $6041-1$ & & $>10^{7} 3.85 \mathrm{E}+06$ & \\
\hline $7041-1$ & & $>10^{7} 5.24 \mathrm{E}+06$ & \\
\hline
\end{tabular}
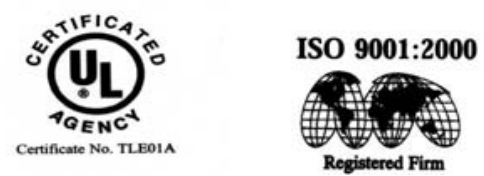

ISO/IEC 17025
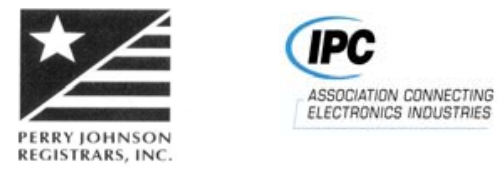


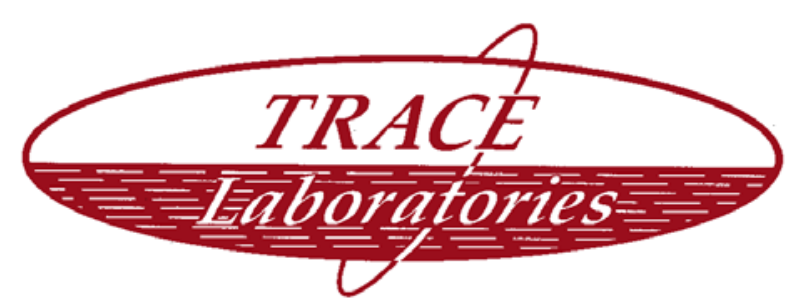

\section{VISUAL}

\section{TEST SPECIMENS:}

Four (4) lots of flexible printed circuit boards

\section{REFERENCE:}

IPC-6013A, Amendment 2, Class 3 paragraphs 3.3.1.2, 3.3.2 - 3.3.2.10.

\section{REQUIREMENT:}

The finished flexible printed wiring shall be examined in accordance with the following procedure. They shall be of uniform quality and shall conform to sections 3.3.1 and 3.3.9.

Visual examination for applicable attributes shall be conducted at 3 diopters (approx. 1.75X). If the acceptable condition of a suspected defect is not apparent, it should be verified at progressively higher magnifications (up to 40X) to confirm that it is a defect. Dimensional requirements such as spacing or conductor width measurements require other magnification and devices with reticles or scales in the instrument which allow accurate measurements of the specified dimensions. Contract or specification may require other magnifications.

\subsubsection{Edges, Rigid Section Nicks, Crazing or haloing along the edges of the flexible printed wiring,} cutouts, and nonplated-through holes are acceptable, provided the penetration does not exceed $50 \%$ of the distance from the edge of the nearest conductor or $2.5 \mathrm{~mm}$ [0.0984 in], whichever is less. Edges shall be clean cut and without metallic burrs. Nonmetalic burrs are acceptable as long as they are not loose and/or do not affect fit and function. Panels that are scored or routed with a breakaway tab shall meet the depanalization requirements of the assembled flexible printed wiring.

3.3.1.2 Edges, Flexible Section The trimmed edges of the flexible printed wiring or the flexible section of finished rigid-flex printed wiring shall be free of burrs, nicks or delamination in excess of that allowed in the procurement documentation. Tears shall not be allowed in Type 1 or Type 2 flexible printed wiring or within flexible sections of Type 3 or Type 4 . When nicks and tears occur as a result of tie-in tabs to facilitate circuit removal, the extent of these imperfections shall be agreed upon between user and supplier. Minimum edge to conductor spacing shall be specified in the procurement documentation.

3.3.1.3 Transition Zone, Rigid Area to Flexible Area The transition zone is the area centered on the edge of the rigid portion from which the flex portion extends. The inspection range is limited to $3 \mathrm{~mm}[0.118$ in], centered on the transition, which is the edge of the rigid portion. Visual imperfections inherent to the fabrication technique (i.e., adhesive squeeze-out, localized deformation of the dielectric and conductors,
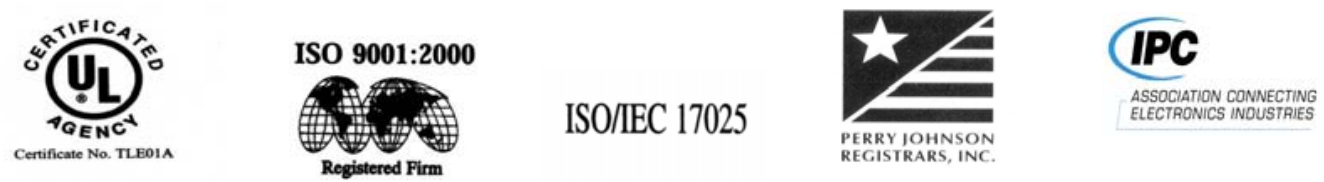


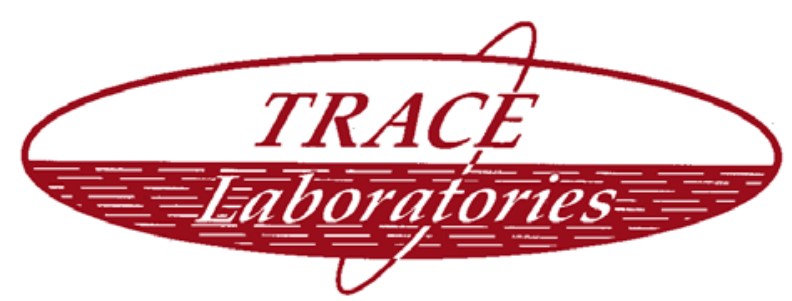

protruding dielectric materials, crazing, or haloing) shall not be cause for rejection. Imperfections in excess of that allowed shall be agreed upon between the fabricator and user, or as so stated on the procurement documentation.

3.3.2 Construction Imperfections Laminate imperfections include those characteristics that are both internal and external within the printed board but are visible from the surface.

3.3.2.1 Measling Measling is acceptable for all classes of end product, with the exception of highvoltage applications. Refer to IPC-A-600 for more information.

3.3.2.2 Crazing Crazing is acceptable for all classes of end product provided the imperfection does not reduce the conductor spacing below the minimum and there is no propagation of the imperfection as a result of thermal testing that replicates the manufacturing process. For Class 2 and 3, the distance of crazing shall not span more than $50 \%$ of the distance between adjacent conductors.

3.3.2.3 Delamination/ Blister Delamination and blistering is acceptable for all classes of end product provided the area affected imperfections does not exceed $1 \%$ of the board area on each side and does not reduce the spacing between conductive patterns below the minimum conductor spacing. There shall be no propagation of imperfections as a result of thermal testing that replicates the manufacturing process. For Class 2 and 3, the blister or delamination shall not span more than $25 \%$ of the distance between adjacent conductive patterns.

3.3.2.4 Foreign Inclusions Translucent particles trapped within the board shall be acceptable. Other particles trapped within the board shall be acceptable, provided the particle does not reduce the spacing between adjacent conductors to below the minimum spacing specified in 3.5.2.

3.3.2.5 W_ eave Exposure Weave exposure or exposed/disrupted fibers are acceptable for all Classes provided the imperfection does not reduce the remaining conductor spacing (excluding the area(s) with weave exposure) below the minimum. Refer to IPC-A-600 for more information.

3.3.2.6 Scratches, Dents, and Tool Marks Scratches, dents and tool marks are acceptable provided they do not expose conductors or expose/disrupt fibers greater than allowed in 3.3.2.4 and 3.3.2.5 and do not reduce the dielectric spacing below the minimum specified. Dents or tool marks that cause delamination, changes physical size of the conductor, or reduces condor width or spacing shall be rejected.

3.3.2.7 Surface Microvoids Surface microvoids are acceptable provided they do not exceed $0.8 \mathrm{~mm}$ [0.0315 in] in the longest dimension, bridge conductors, or exceed $5 \%$ of the total flexible printed wiring area.
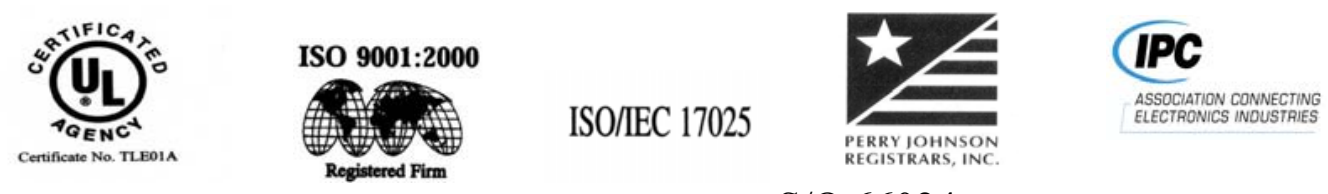


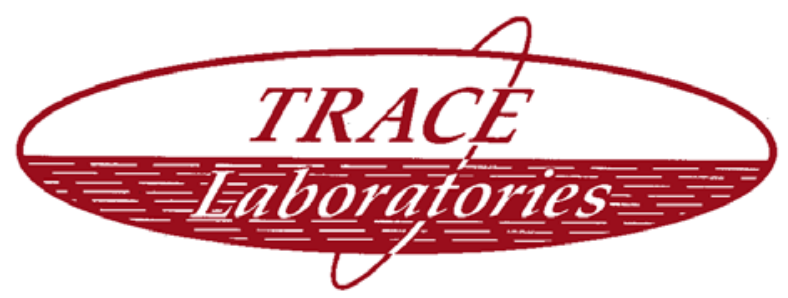

3.3.2.8 Color Variations in Bond Enhancement Treatment Mottled appearance or color variation in bond enhancement treatment is acceptable. Random missing areas of treatment shall not exceed $10 \%$ of the total conductor surface area of the affected layer.

3.3.2.9 Pink Ring There is no existing evidence that pink ring affects functionality. The presence of pink ring may be considered an indicator of process or design variation but is not a cause for rejection. The focus of concern should be the quality of the lamination bond.

3.3.2.10 Coverfilm Separations The coverfilm shall be uniform and free of coverfilm separations, such as wrinkles, creases, and soda strawing. Nonlamination shall be acceptable, provided such imperfections do not violate 3.3.2.4 and all of the following:

a. At random locations away from conductors, if each separation is no larger than $0.80 \mathrm{~mm} \mathrm{x}$ $0.80 \mathrm{~mm}$ [0.0315 in x $0.0315 \mathrm{in}]$ and is not within $1.0 \mathrm{~mm}$ [0.0394 in] of the board edge or the coverfilm opening. The total number of separations shall not exceed three in any $25 \mathrm{~mm}$ x $25 \mathrm{~mm}$ [0.984 in x 0.984 in] of coverfilm surface area.

b. The total separation shall not exceed $25 \%$ of the spacing between adjacent conductors.

c. There shall be no coverfilm nonlamination along the outer edges or covercoat openings of the coverfilm that reduces the seal below minimum edge to conductor spacing.

3.3.2.11.1 Covercoat Coverage Covercoat coverage manufacturing variations resulting in skips, voids, and misregistration are subject to the following restrictions:

a. Metal conductors shall not be exposed or bridged by blisters in areas where covercoat is required. Touch up, if required to cover these areas with covercoat, shall be of a material that is compatible and of equal resistance to soldering and cleaning as the originally applied covercoat.

b. In areas containing parallel conductors, covercoat variations shall not expose adjacent conductors unless the area between the conductors is purposely left blank as for a test point or for some surface mount devices.

c. Covercoat need not be flush with the surface of the land. Misregistration of a covercoatdefined feature shall not expose adjacent isolated lands or conductors.

d. Covercoat is allowed on lands for plated through holes to which solder connections are to be made, provided the external annular ring requirements for that class of products are not violated. Resist shall not encroach upon the barrel of this type of plated through holes.
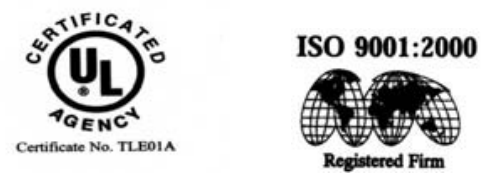

ISO/IEC 17025
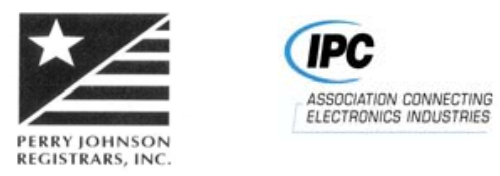


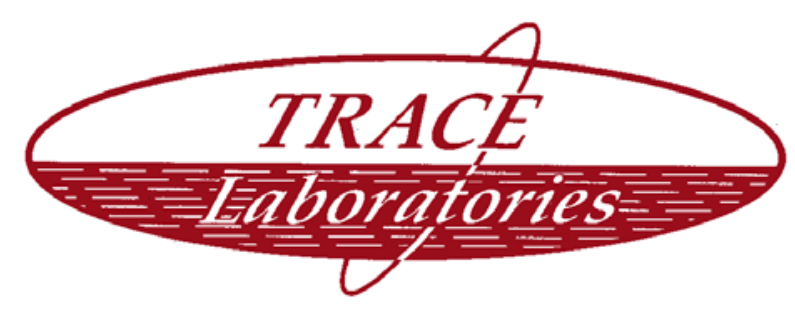

Other surfaces, such as edge flexible printed wiring connector fingers and surface mount lands, shall be free of covercoat, except as specified. Covercoat allowed in plated through holes and vias into which no component lead is soldered, unless the procurement documentation requires that the holes be completely solder filled. Covercoat may tent or plug via holes as specified by the procurement documentation. Test points that are intended for assembly testing must be free of covercoat unless coverage is specified.

e. When a land contains no plated through holes, as in the case of surface mount or ball grid array (BGA) lands, misregistration shall not cause encroachment of the covercoat on the land or lack of solder resist definition in excess of the following:

1) On surface mount lands, misregistration shall not cause encroachment of the covercoat over the land area greater than $0.050 \mathrm{~mm}$ [0.00197 in] for a pitch $\geq 1.25 \mathrm{~mm}$ [0.04921 in]. Encroachment shall not exceed $0.025 \mathrm{~mm}$ [0.00098 in] for a pitch $\leq 1.25 \mathrm{~mm}$ [0.04921 in] and encroachment may occur on adjacent sides, but not on opposite sides of a surface mount land.

2) On BGA lands, if the land is solder resist defined misregistration may allow a $90^{\circ}$ breakout of the covercoat on the land. If clearance is specified, no encroachment of the covercoat on the land is allowed, except at the conductor attachment.

3) On BGA lands connected to via holes, which have coverlayer dams required, the dam shall be continuous without missing peeling or cracked coverlayer, allowing a bare metal path between the BGA land and the via.

f. Blistering shall be allowed to the following extent:

1) Class 1: Does not bridge between conductors.

2) Class 2 and 3: Two per side, maximum $0.25 \mathrm{~mm}$ [0.00984 in] in longest dimension, does not reduce electrical spacing between conductors by more than $25 \%$.

g. Pits and voids are allowed in nonconductor areas, provided they have adherent edges and do not exhibit lifting or blistering in excess of allowance in 3.3.2.11.1(f).

h. Coverage between closely spaced surface mount lands shall be as required by procurement documentation.
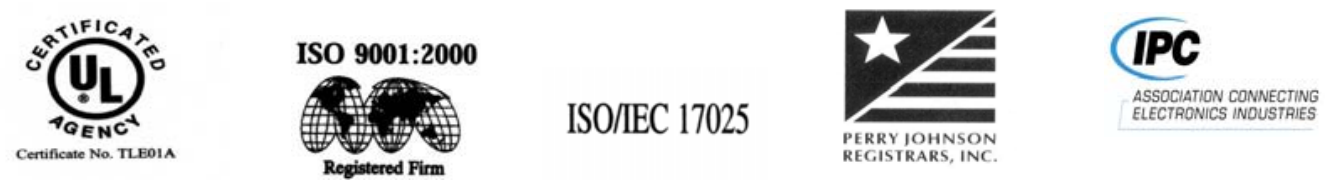


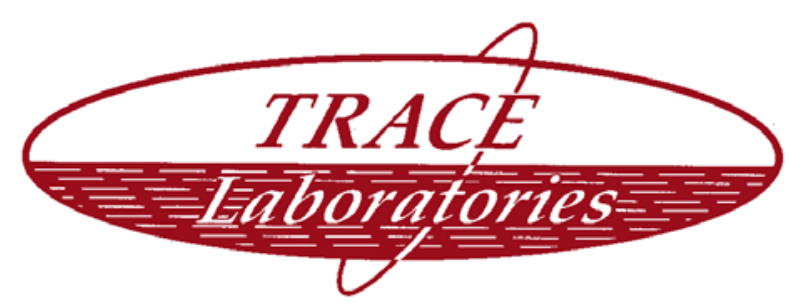

i. When design requires coverage to the flexible printed wiring edge, chipping or lifting of covercoat along the flexible printed wiring edge after fabrication shall not penetrate more than $1.25 \mathrm{~mm}$ [ $0.04291 \mathrm{in}]$ or $50 \%$ of the distance to the closest conductor, whichever is less.

3.3.2.11.2 Covercoat Cure and Adhesion The cured covercoat shall not exhibit tackiness or blistering in excess of that permitted in 3.3.2.11.1(f). When tested in accordance with IPC-TM-650, Method 2.4.28.1, the maximum percentage of cured covercoat lifting from Coupon G identified in IPC-2221 shall be in accordance with Table 3-1.

Table 3-1 Covercoat Adhesion

\begin{tabular}{|l|c|c|c|}
\hline \multirow{2}{*}{ Surface } & \multicolumn{3}{|c|}{ Maximum Percentage Loss Allowed } \\
\cline { 2 - 4 } & Class 1 & Class 2 & Class 3 \\
\hline Bare Copper & 10 & 5 & 0 \\
\hline Gold Nickel & 25 & 10 & 5 \\
\hline Base Laminate & 10 & 5 & 0 \\
\hline $\begin{array}{l}\text { Melting Metals } \\
\text { (Tin-lead, fused tin- } \\
\begin{array}{l}\text { lead, and bright } \\
\text { acid-tin) }\end{array}\end{array}$ & 50 & 25 & 10 \\
\hline
\end{tabular}

\subsection{Covercoat Thickness Covercoat thickness is not measured, unless specified on the} procurement documentation. If a thickness measurement is required, instrumental methods may be used or an assessment may be made using a microsection of the parallel conductors on Coupon E identified in IPC-2221.

3.3.2.12 Solder Wicking/Plating Penetration Solder wicking or other plating penetration shall not extend into a bend or flex transition area and shall meet the conductor spacing requirements. Solder wicking or other plating penetration shall not exceed the limits specified in Table 3-2.

Table 3-2 Solder Wicking/Plating Penetration Limits

\begin{tabular}{|c|c|c|}
\hline Class 1 & Class 2 & Class 3 \\
\hline $\begin{array}{c}\text { As agreed upon between } \\
\text { user and supplier }\end{array}$ & $0.5 \mathrm{~mm}(0.020$ ") maximum & $0.3 \mathrm{~mm}(0.012$ ') maximum \\
\hline
\end{tabular}

3.3.2.13 Stiffener A stiffener will be evaluated only as a mechanical support. Void free bonding of the stiffener to the flexible printed wiring is not required. Specific requirements shall be as agreed upon between user and supplier.
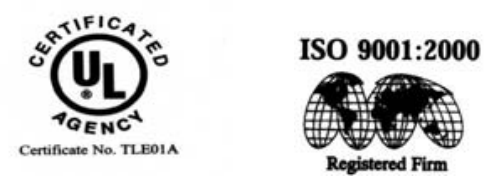

ISO/IEC 17025
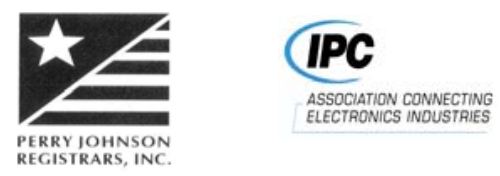


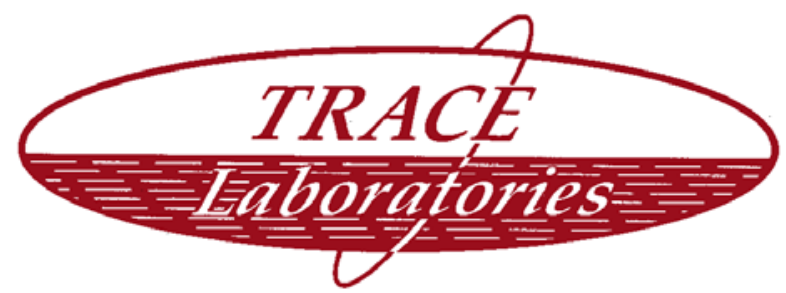

3.3.1 Plating and Coating Voids in the Hole Plating and coating voids shall not exceed that allowed by Table $3-3$.

Table 3-3 Plating and Coating Voids Visual Examination

\begin{tabular}{|l|l|l|l|}
\hline \multicolumn{1}{|c|}{ Material } & \multicolumn{1}{c|}{ Class 1 } & \multicolumn{1}{c|}{ Class 2 } & \multicolumn{1}{c|}{ Class 3 } \\
\hline Copper $^{1}$ & $\begin{array}{l}\text { Three voids allowed } \\
\text { per hole in not more } \\
\text { than } 10 \% \text { of the holes. }\end{array}$ & $\begin{array}{l}\text { One void allowed per } \\
\text { hole in not more than } \\
5 \% \text { of the holes. }\end{array}$ & None \\
\hline Finish Coating $^{2}$ & $\begin{array}{l}\text { Five voids allowed per } \\
\text { hole in not more than } \\
15 \% \text { of the holes. }\end{array}$ & $\begin{array}{l}\text { Three voids allowed } \\
\text { per hole in not more } \\
\text { than 5\% of the holes. }\end{array}$ & $\begin{array}{l}\text { One void allowed per } \\
\text { hole in not more than } \\
5 \% \text { of the holes. }\end{array}$ \\
\hline
\end{tabular}

${ }^{1}$ For class 2 flexible printed wiring product, copper voids shall not exceed $5 \%$ of the hole length. For class 1 flexible printed wiring product, copper voids shall not exceed $10 \%$ of the hole length. Circumferential voids shall not extend beyond $90^{\circ}$ of the circumference.

${ }^{2}$ For class 2 and 3 product, finished coating voids shall not exceed $5 \%$ of the hole length. For class 1 , finished coating voids shall not exceed $10 \%$ of the hole length. Circumferential voids shall not extend beyond $90^{\circ}$ for class 1,2 , or 3 .

3.3.4 Markings If required, each individual flexible printed board, qualification flexible printed board, and set of quality conformance test circuit strips (as opposed to each individual test coupon) shall be marked. This marking is required in order to insure traceability between the flexible printed wiring/test strips and the manufacturing history and to identify the supplier (logo, etc.). If size or space does not permit marking individual flexible printed wiring, bagging or tagging is permitted.

The marking shall be produced by the same process as used in producing the conductive pattern, or by use of a permanent fungistatic ink or paint (see 3.2.10), LASER marker or by vibrating pencil marking on a metallic area provided for marking purposes.

Conductive markings, either etched copper or conductive ink (see 3.2.10) shall be considered as electrical elements of the circuit and shall not reduce the electrical spacing requirements. All markings shall be compatible with materials and parts, legible for all tests, and in no case affect flexible printed wiring performance.

Marking shall not cover areas of lands that are to be soldered. (see IPC-A-600 for legibility requirements). In addition to this marking, the use of bar code marking is permissible. When used, date code shall be formatted per the supplier's discretion in order to establish traceability as to when the manufacturing operations were performed.

3.3.5 Solderability Only those flexible printed boards that require soldering in a subsequent assembly operation require solderability testing. Solderability testing is not necessary for flexible printed wiring that does not require soldering. This shall be specified on the master drawing, as in the case where press-
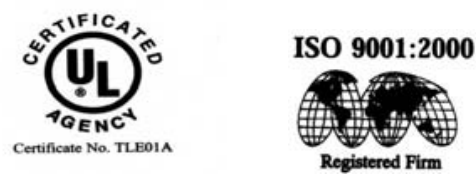

ISO/IEC 17025
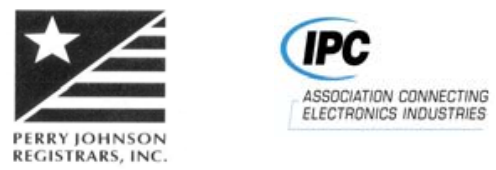


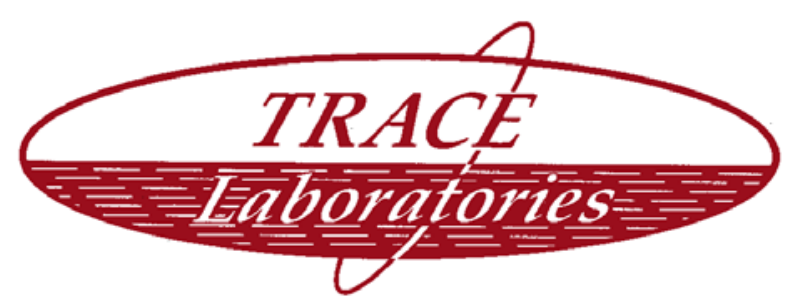

fit components are used. Flexible printed wiring to be used for surface mount does not require hole solderability testing.

When required by the procurement documentation, accelerated aging for coating durability shall be in accordance with J-STD-003. The Category of durability shall be specified on the master drawing; however, in not specified, Category 2 shall be used. Specimens to be tested shall be conditioned, if required, and evaluated for surface and hole solderability using J-STD-003.

When solderability testing is required, consideration should be given to flexible printed wiring thickness and copper thickness. As both increase, the amount of time to properly wet the sides of the holes and the tops of the lands increases proportionately.

Note: Accelerated aging (steam aging) is intended for use on coatings of tin/lead solder or tin, but not other final finishes.

3.3.6 Plating Adhesion The adhesion of the plating shall be tested in accordance with IPC-TM-650, method 2.4.1. There shall be no evidence of any portion of the protective plating or the conductor pattern foil being removed, as shown by particles of the plating or pattern foil adhering to the tape. If overhanging metal (slivers) breaks off and adheres to the tape, it is evidence of overhang or slivers, but not of plating adhesion failure.

\subsubsection{Edge Board Contact, Junction of Gold Plate to Solder Finish Exposed copper/plating overlap} between the solder finish and gold plate shall meet the requirements of Table 3-4. The exposed copper /plating or gold overlap may exhibit a discolored or gray-black area which is acceptable (see 3.5.3.3).

Table 3-4 Edge Board Contact Gap

\begin{tabular}{|c|c|c|}
\hline Class & Maximum Exposed Copper Gap & Maximum Gold Overlap \\
\hline 1 & $2.5 \mathrm{~mm}[0.0984 \mathrm{in}]$ & $2.5 \mathrm{~mm}[0.0984 \mathrm{in}]$ \\
\hline 2 & $1.25 \mathrm{~mm}[0.04921 \mathrm{in}]$ & $1.25 \mathrm{~mm}[0.04921 \mathrm{in}]$ \\
\hline 3 & $0.8 \mathrm{~mm}[0.031 \mathrm{in}]$ & $0.8 \mathrm{~mm}[0.031 \mathrm{in}]$ \\
\hline
\end{tabular}

3.3.8 Lifted Lands When visually examined in accordance with 3.3, there shall be no lifted lands on the delivered (nonstressed) printed circuit board.

3.3.9 W_ orkmanship Flexible printed boards shall be processed in such a manner as to be uniform in quality and show no visual evidence of dirt, foreign matter, oil, fingerprints, tin/lead or solder smear transfer to the dielectric surface, flux residue and other contaminants that affect life, ability to assemble and serviceability. Visually dark appearances in non-plated holes, which are seen when a metallic or non-
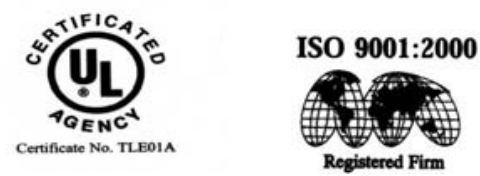

ISO/IEC 17025
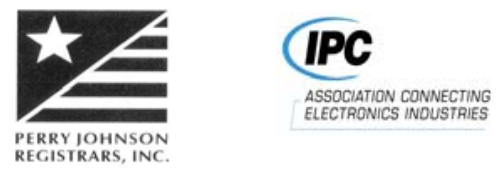


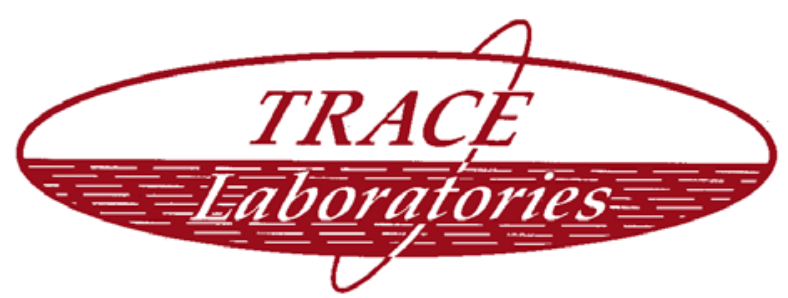

metallic semiconductive coating is used, are not foreign material and do not affect life or function. Flexible printed wiring shall be free of defects in excess of those allowed in this specification. There shall be no evidence of any lifting or separation of platings from the surface of the conductive pattern, or of the conductor from the base laminate in excess of that allowed. There shall be no loose plating slivers on the surface of the printed board.

\section{METHOD:}

The boards were visually examined with approximately $1.75 \mathrm{X}$ using various light sources. Progressively higher magnifications, up to $40 \mathrm{X}$, were used for referee evaluation.

\section{RESULTS:}

The boards met the requirements of IPC-6013A, Amendment 2, Class 3, paragraph 3.3.

\section{THERMAL SHOCK}

\section{TEST SPECIMENS:}

Four (4) lots of flexible printed circuit boards.

\section{REFERENCE:}

IPC-6013A, Amendment 2, Class 3, paragraph 3.10.2.

\section{REQUIREMENT:}

When specified on the procurement documentation, flexible printed wiring or test coupons shall be tested in accordance with the procedure outlined below.

The specimen shall be tested for thermal shock in accordance with IPC-TM-650, method 2.6.7.2B, except the temperature range shall be $-65^{\circ} \mathrm{C}$ to $125^{\circ} \mathrm{C}\left[-85^{\circ} \mathrm{F}\right.$ to $\left.257^{\circ} \mathrm{F}\right]$. Microsection evaluation in accordance with IPC-TM-650, Method 2.6.7.2 is not required. Following removal from the test chamber, the specimen shall meet the circuitry requirements of 3.9.2. The resistance value shall not vary by more than \pm $10 \%$.

\section{METHOD:}

Insulated wires were soldered to the terminals of the test specimen. The initial resistance measurement was obtained using a multimeter. The samples were placed in Thermal Shock
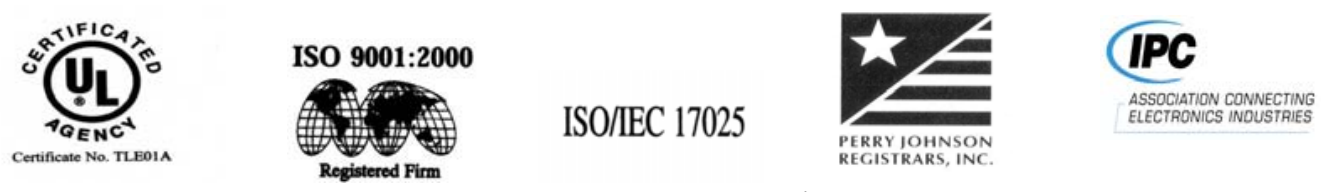


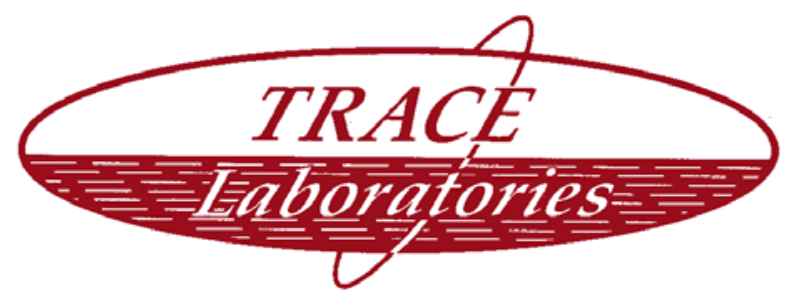

Chamber. The cold chamber was set at $-65^{\circ} \mathrm{C}$, and the hot portion of the chamber was set at $125^{\circ} \mathrm{C}$. The dwell time was set at 15 minutes. The transfer to temperature extremes was approximately 15 seconds. During the first hot cycle, the resistance measurement was obtained with a multimeter.

The chamber was set for 100 cycles. During the last hot cycle the resistance measurement was again measured and recorded. The percent change was then calculated.

\section{RESULTS:}

Resistance Measurements:

Part Number

\begin{tabular}{|c|c|c|c|c|}
\hline & Layer & $\begin{array}{c}\text { Before } \\
\text { Conditioning } \\
\text { (ohms) }\end{array}$ & $\begin{array}{c}\text { After } \\
\text { Conditioning } \\
\text { (ohms) }\end{array}$ & \% Change \\
\hline & & 0.017 & 0.018 & 5.88 \\
\hline & & 0.022 & 0.023 & 4.55 \\
\hline & & 0.016 & 0.016 & 0.00 \\
\hline & & 0.010 & 0.010 & 0.00 \\
\hline & & 0.005 & 0.005 & 0.00 \\
\hline & & 0.027 & 0.028 & 3.70 \\
\hline & & 0.009 & 0.009 & 0.00 \\
\hline & & 0.010 & 0.010 & 0.00 \\
\hline
\end{tabular}

Part Number

\begin{tabular}{|c|c|c|c|c|}
\hline \multirow{3}{*}{$\mathrm{S} / \mathrm{n}$} & Layer & $\begin{array}{c}\text { Before } \\
\text { Conditioning } \\
\text { (ohms) }\end{array}$ & $\begin{array}{c}\text { After } \\
\text { Conditioning } \\
(\mathrm{ohms})\end{array}$ & \% Change \\
\hline & & 0.041 & 0.044 & 7.32 \\
\hline & & 0.040 & 0.041 & 2.50 \\
\hline & & 0.039 & 0.041 & 5.13 \\
\hline & & 0.043 & 0.043 & 0.00 \\
\hline & & 0.044 & 0.043 & -2.27 \\
\cline { 2 - 5 } & & 0.053 & 0.055 & 3.77 \\
\hline & & 0.030 & 0.031 & 3.33 \\
\hline
\end{tabular}
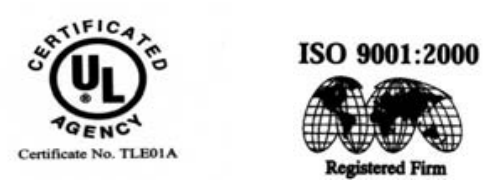

ISO/IEC 17025
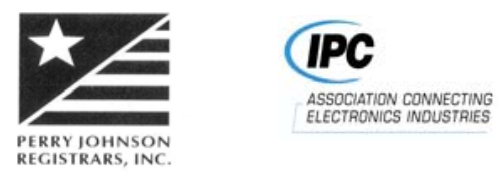


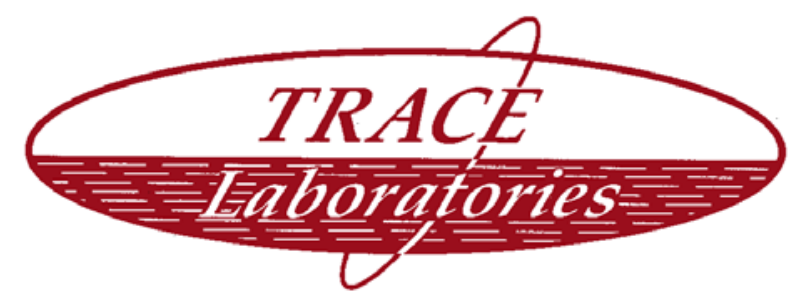

Part Number

\begin{tabular}{|c|c|c|c|c|}
\hline & Layer & $\begin{array}{c}\text { Before } \\
\text { Conditioning } \\
\text { (ohms) }\end{array}$ & $\begin{array}{c}\text { After } \\
\text { Conditioning } \\
\text { (ohms) }\end{array}$ & \% Change \\
\hline $\mathrm{S} / \mathrm{n}$ & & 0.006 & 0.006 & 0.00 \\
\hline & & 0.019 & 0.018 & -5.26 \\
\hline & & 0.008 & 0.008 & 0.00 \\
\hline & & 0.013 & 0.014 & 7.69 \\
\hline
\end{tabular}

Part Number

\begin{tabular}{|c|c|c|c|c|}
\hline \multirow{3}{*}{$\mathrm{S} / \mathrm{n}$} & Layer & $\begin{array}{c}\text { Before } \\
\text { Conditioning } \\
\text { (ohms) }\end{array}$ & $\begin{array}{c}\text { After } \\
\text { Conditioning } \\
\text { (ohms) }\end{array}$ & \% Change \\
\cline { 2 - 5 } & & 0.380 & 0.386 & 1.58 \\
\hline & & 0.350 & 0.365 & 4.29 \\
\hline & & 0.350 & 0.355 & 1.43 \\
\hline
\end{tabular}

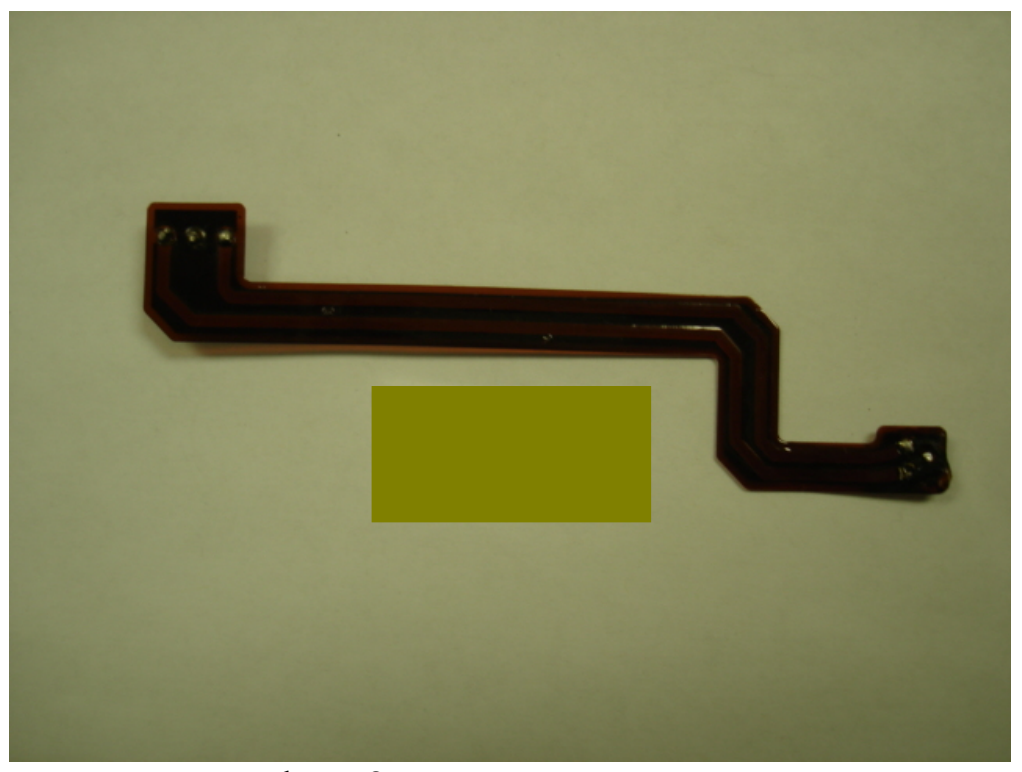

Photo 2:

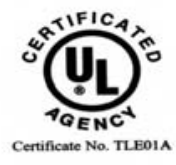



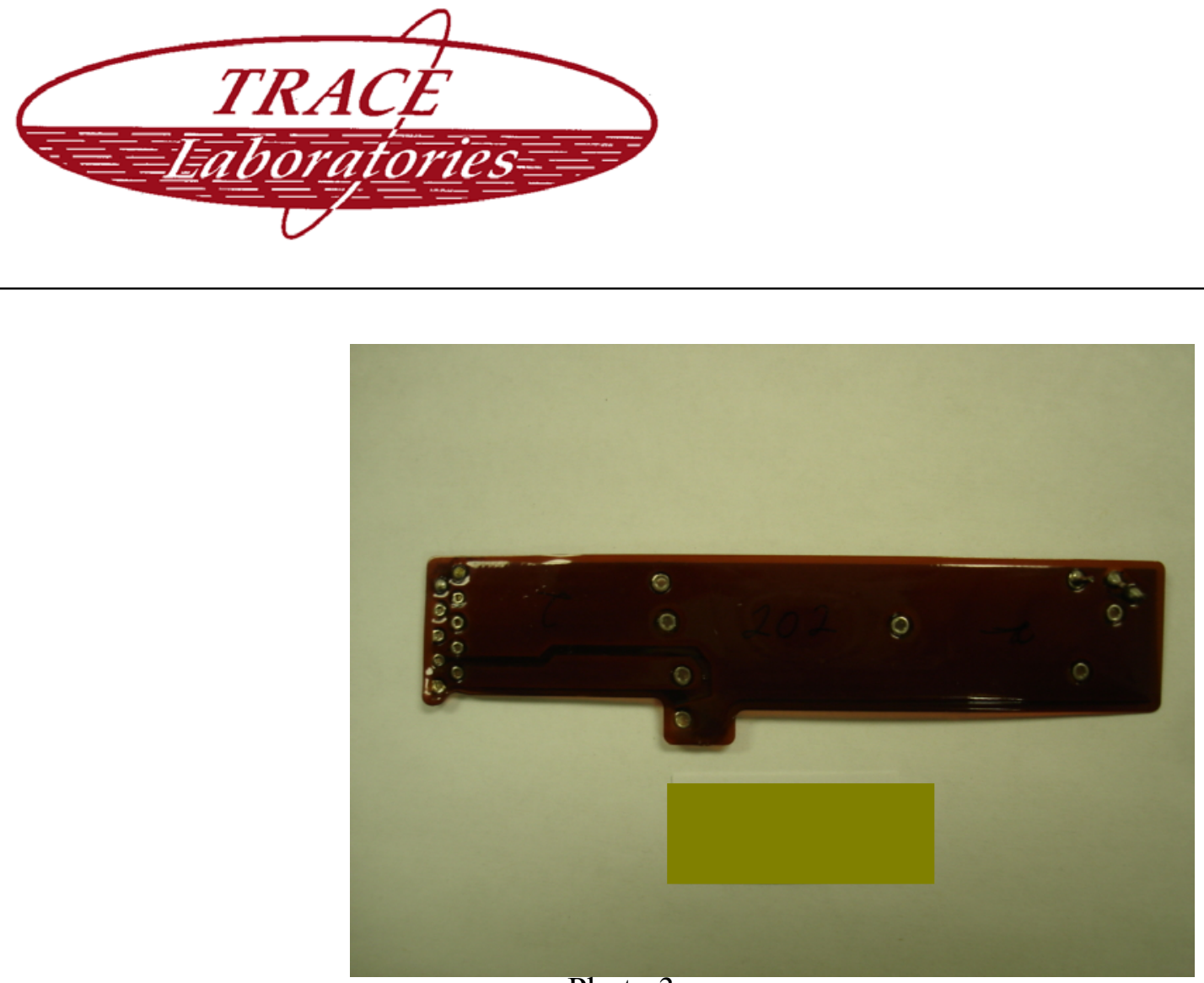

Photo 3:

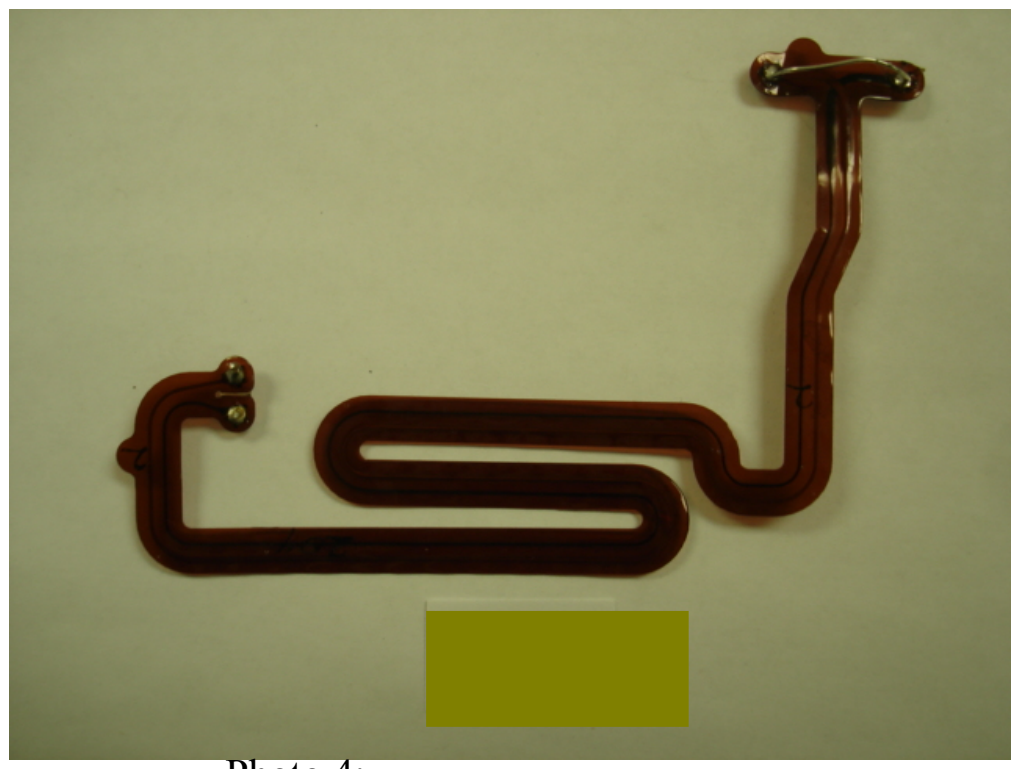

Photo 4:

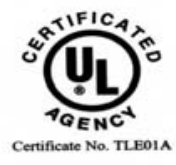

ISO 9001:2000

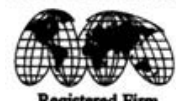

ISO/IEC 17025

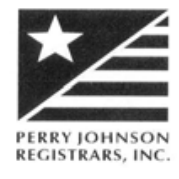

IPC

Registered Firm

S/O 66024 

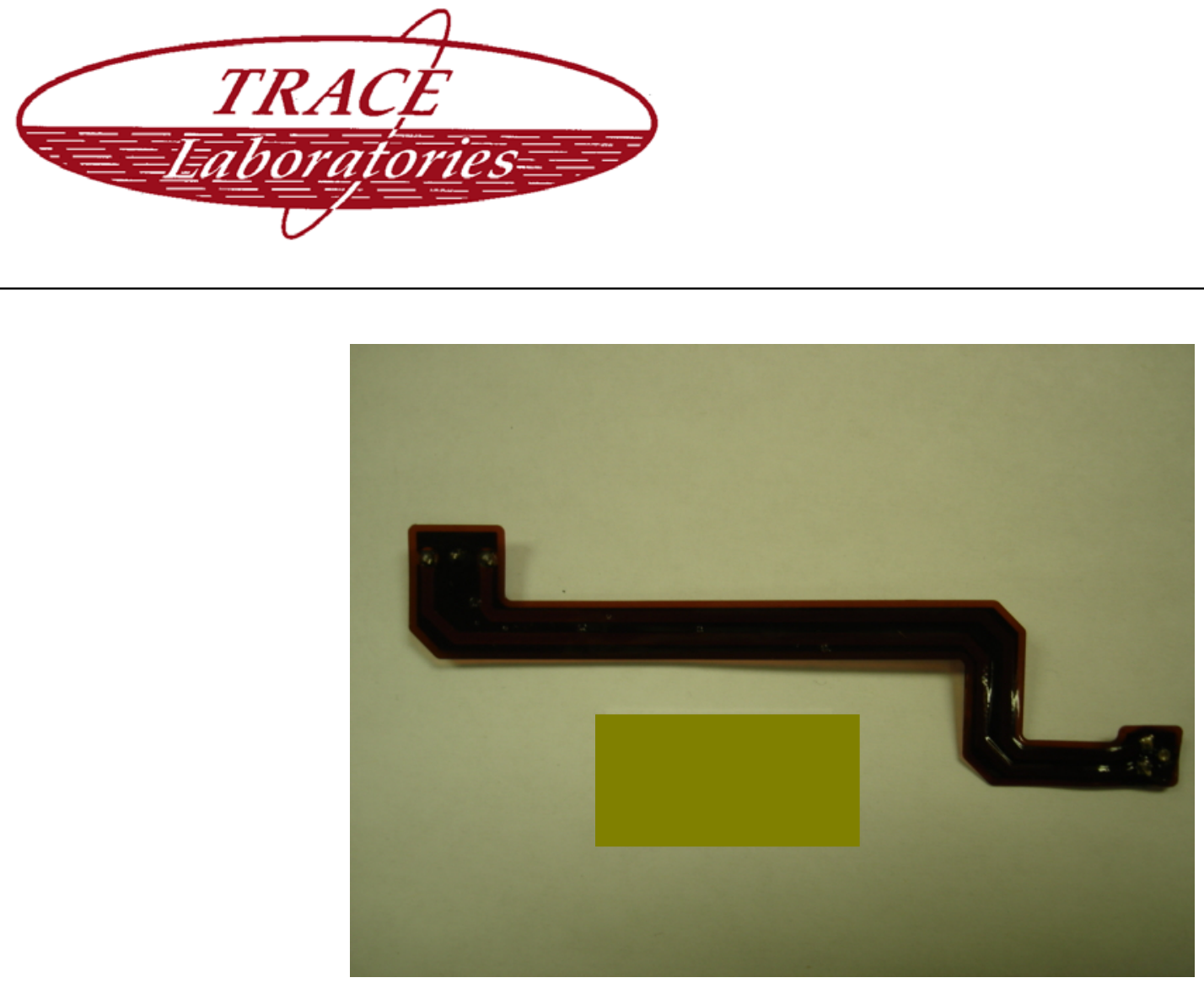

Photo 5:

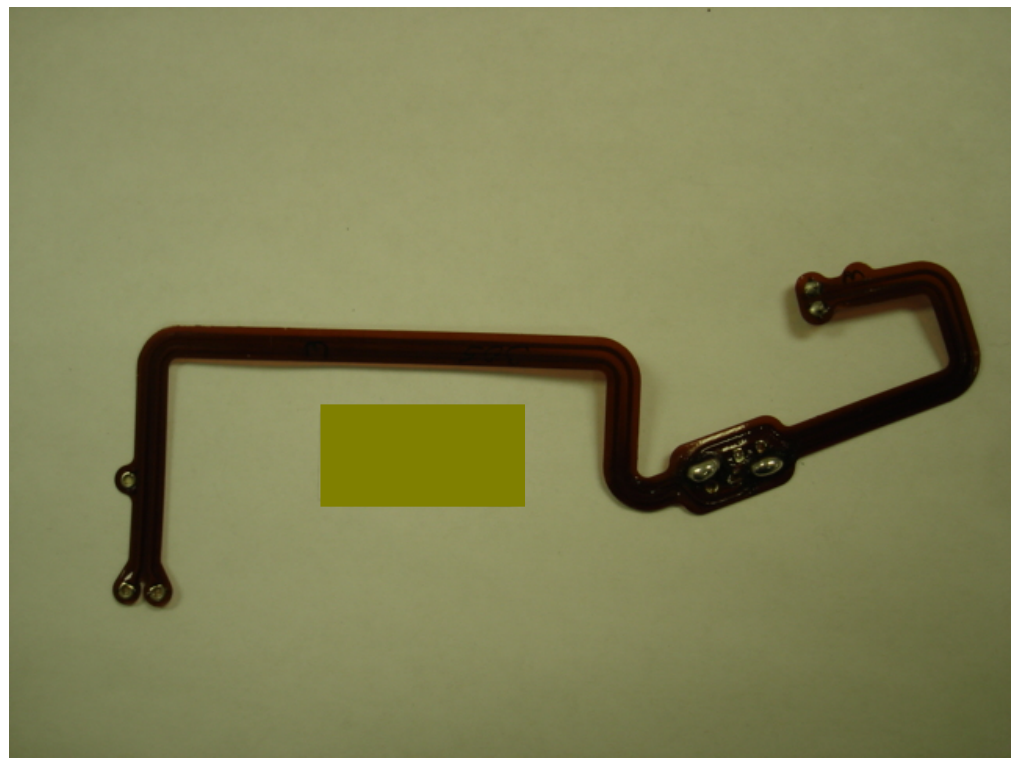

Photo 6:

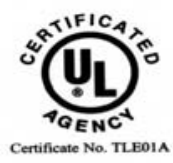

ISO 9001:2000

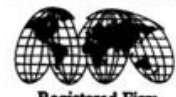

ISO/IEC 17025

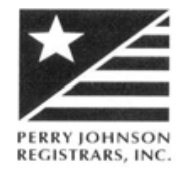

IPC

Registered Firm

S/O 66024 

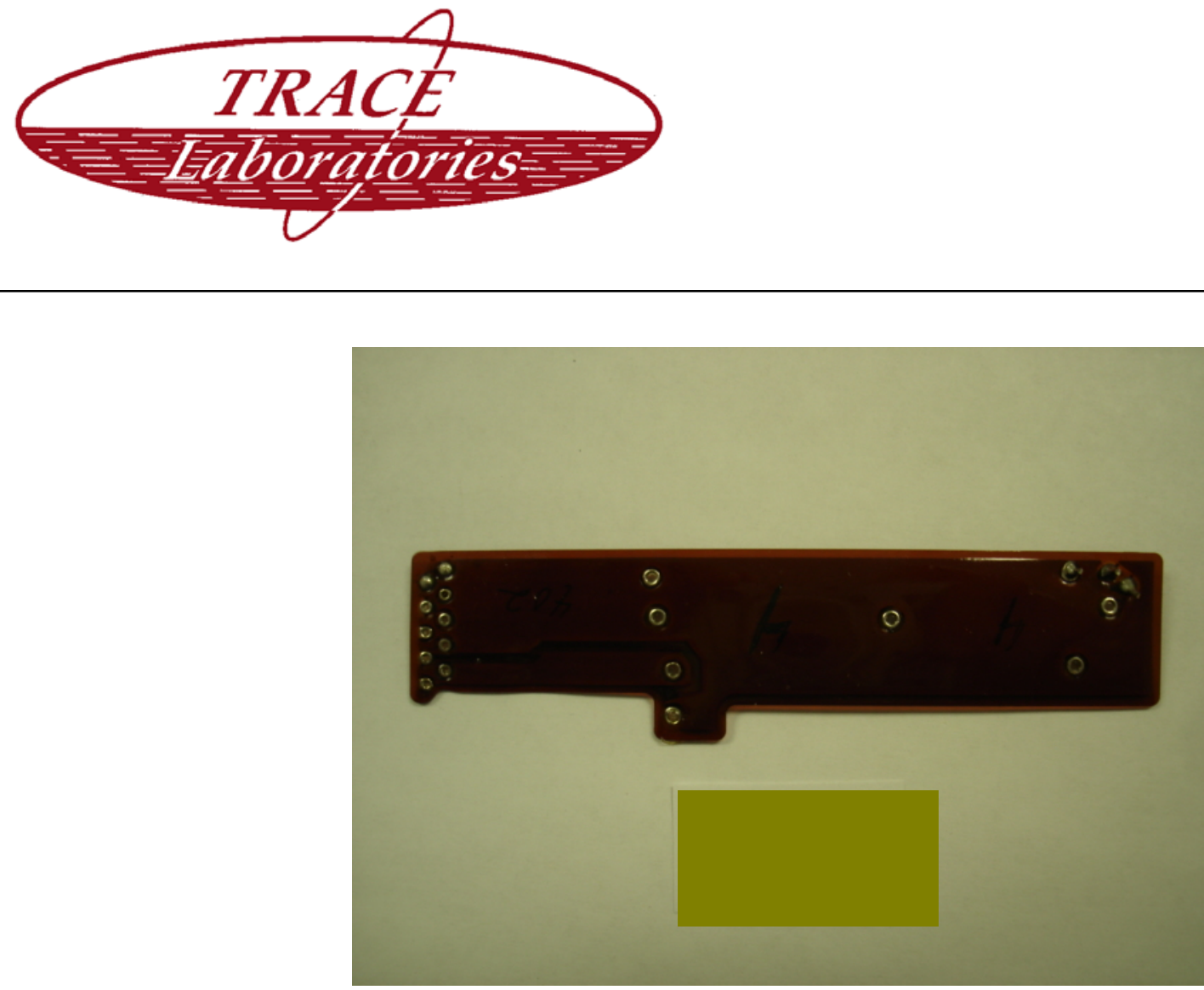

Photo 7:

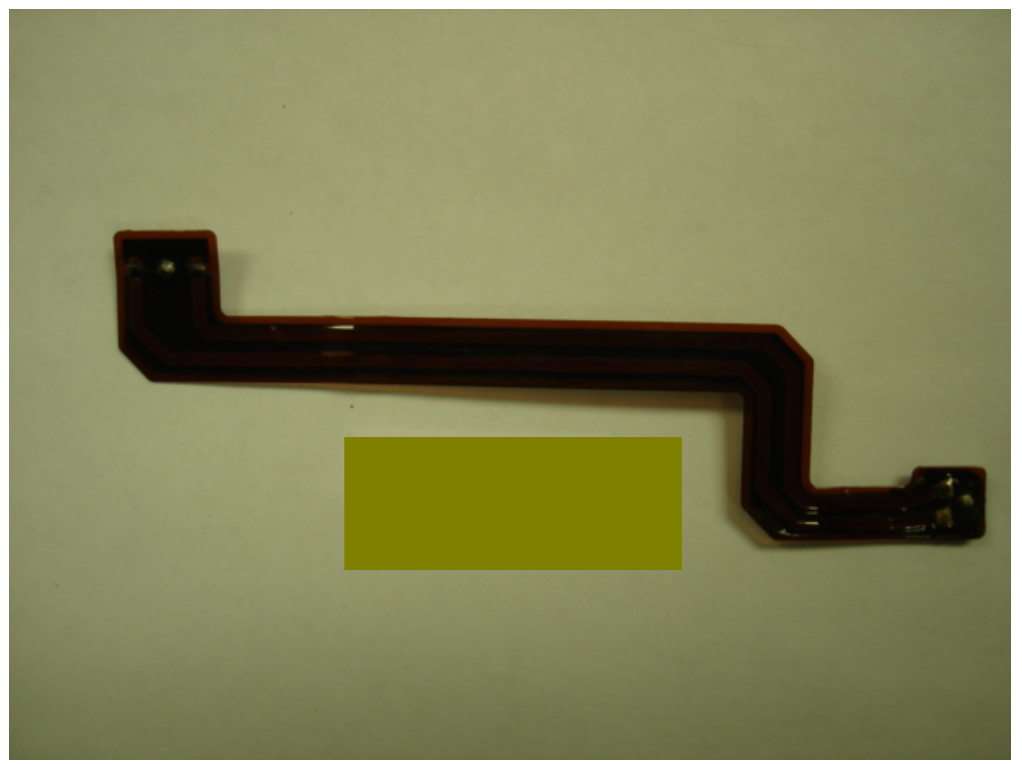

Photo 8:

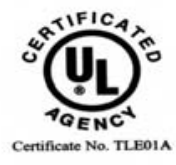

ISO 9001:2000

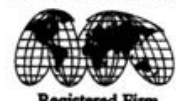

ISO/IEC 17025

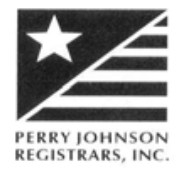

IPC

Registered Firm

S/O 66024 

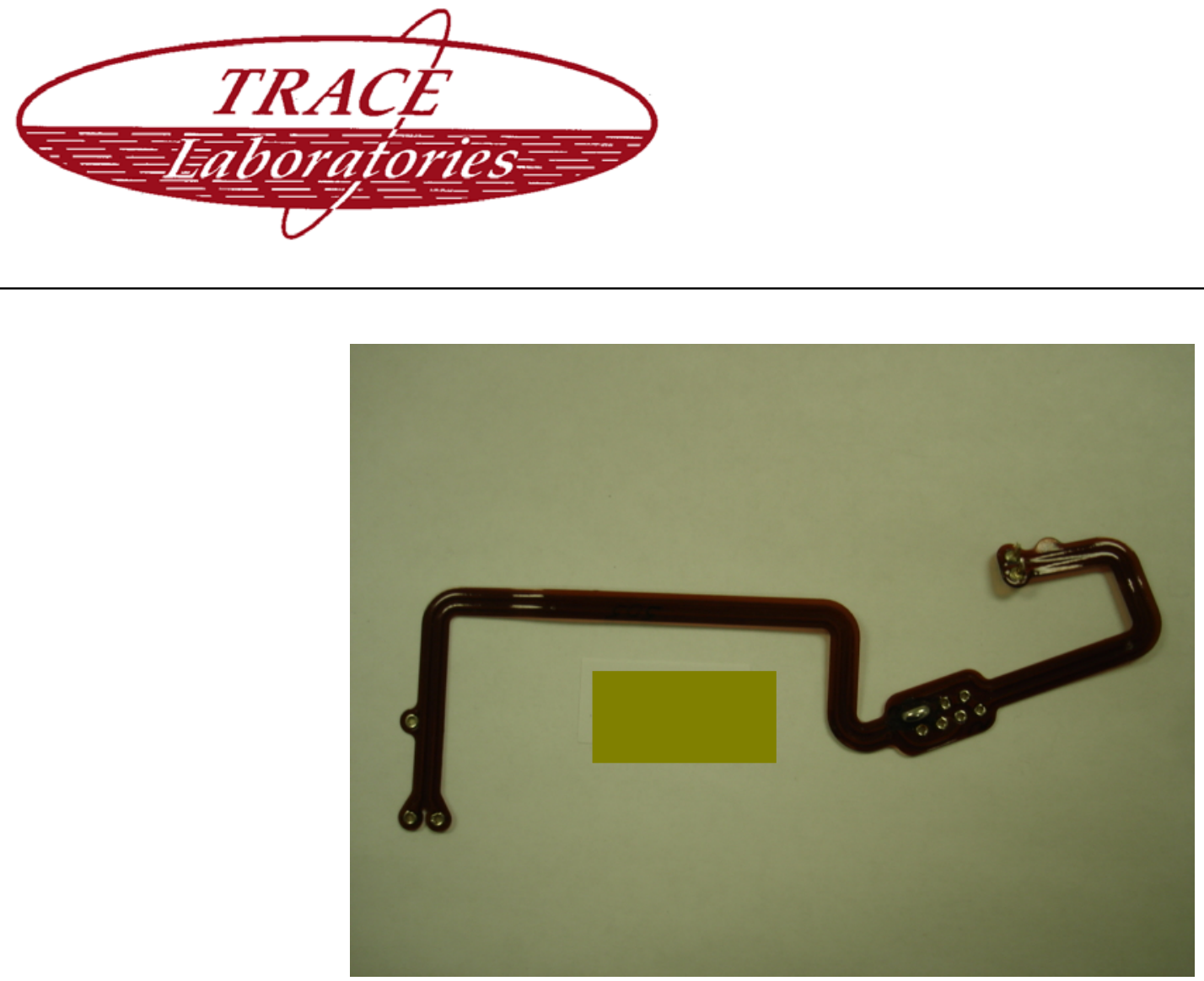

Photo 9:

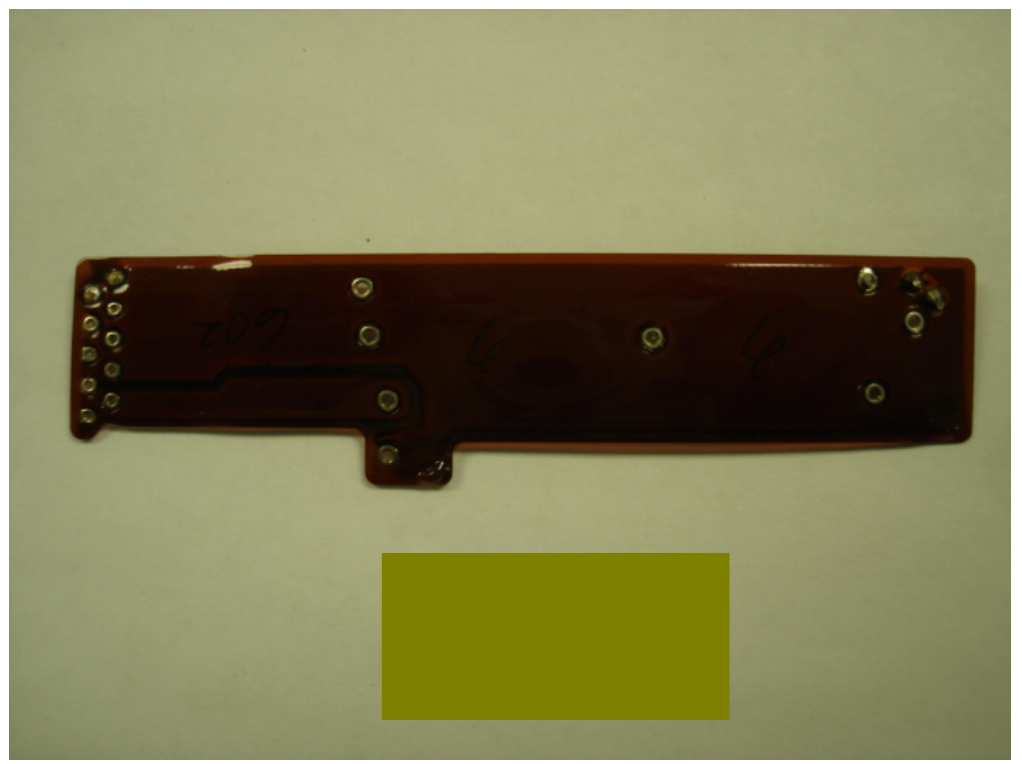

Photo 10:

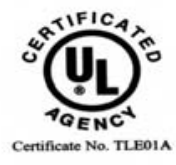

ISO 9001:2000

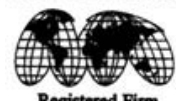

ISO/IEC 17025

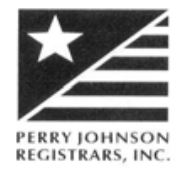

IPC

Registered Firm

S/O 66024 

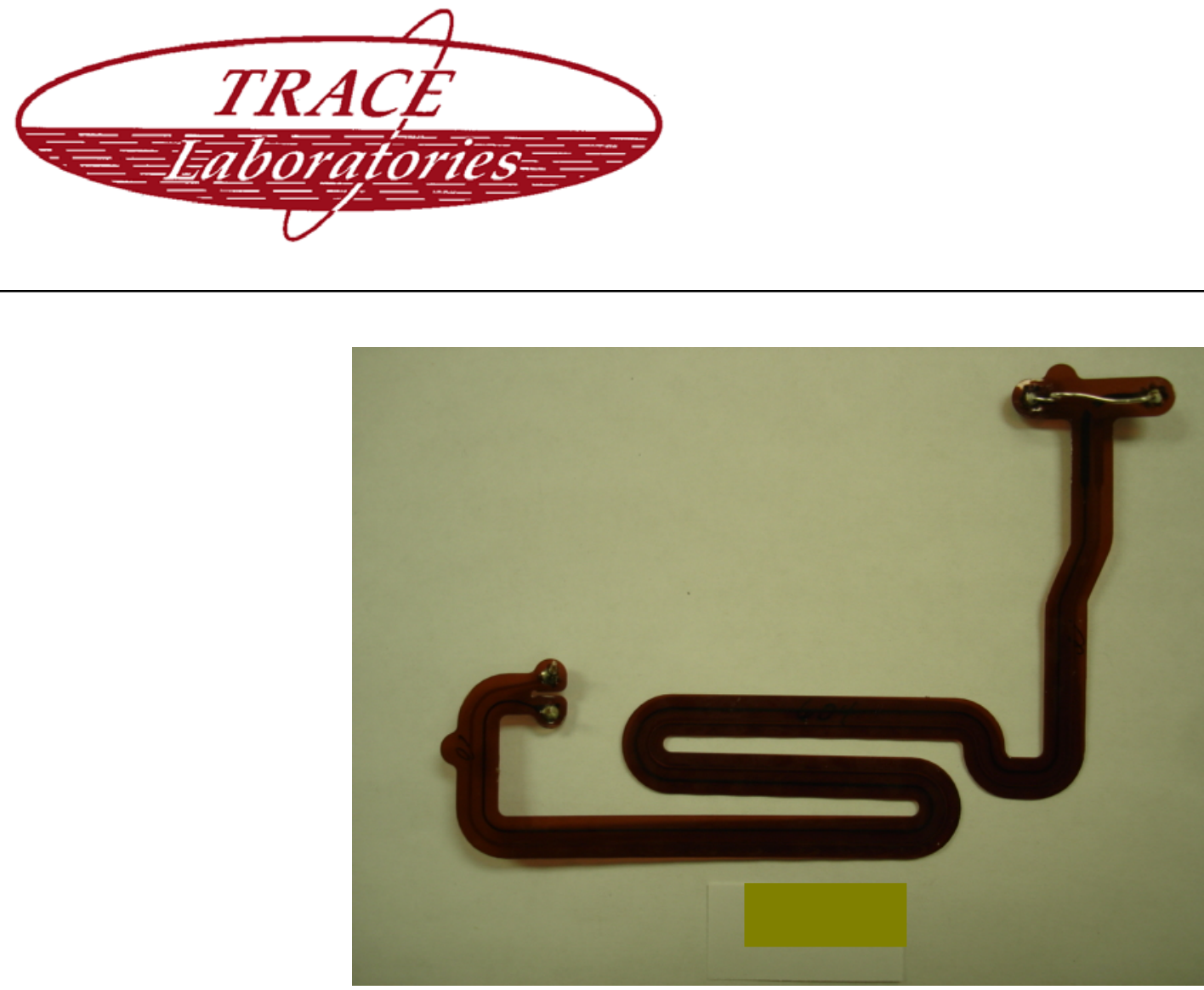

Photo 11:

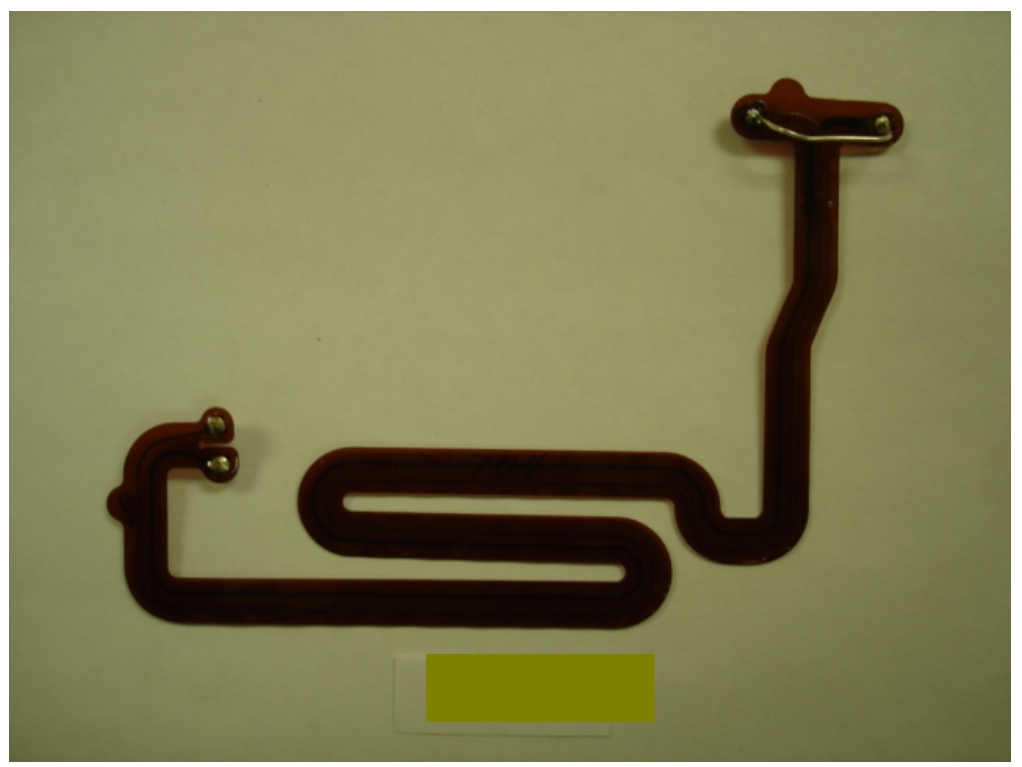

Photo 12:

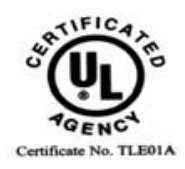

ISO 9001:2000

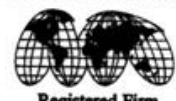

ISO/IEC 17025

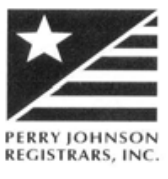

IPC

Registered Firm

S/O 66024 

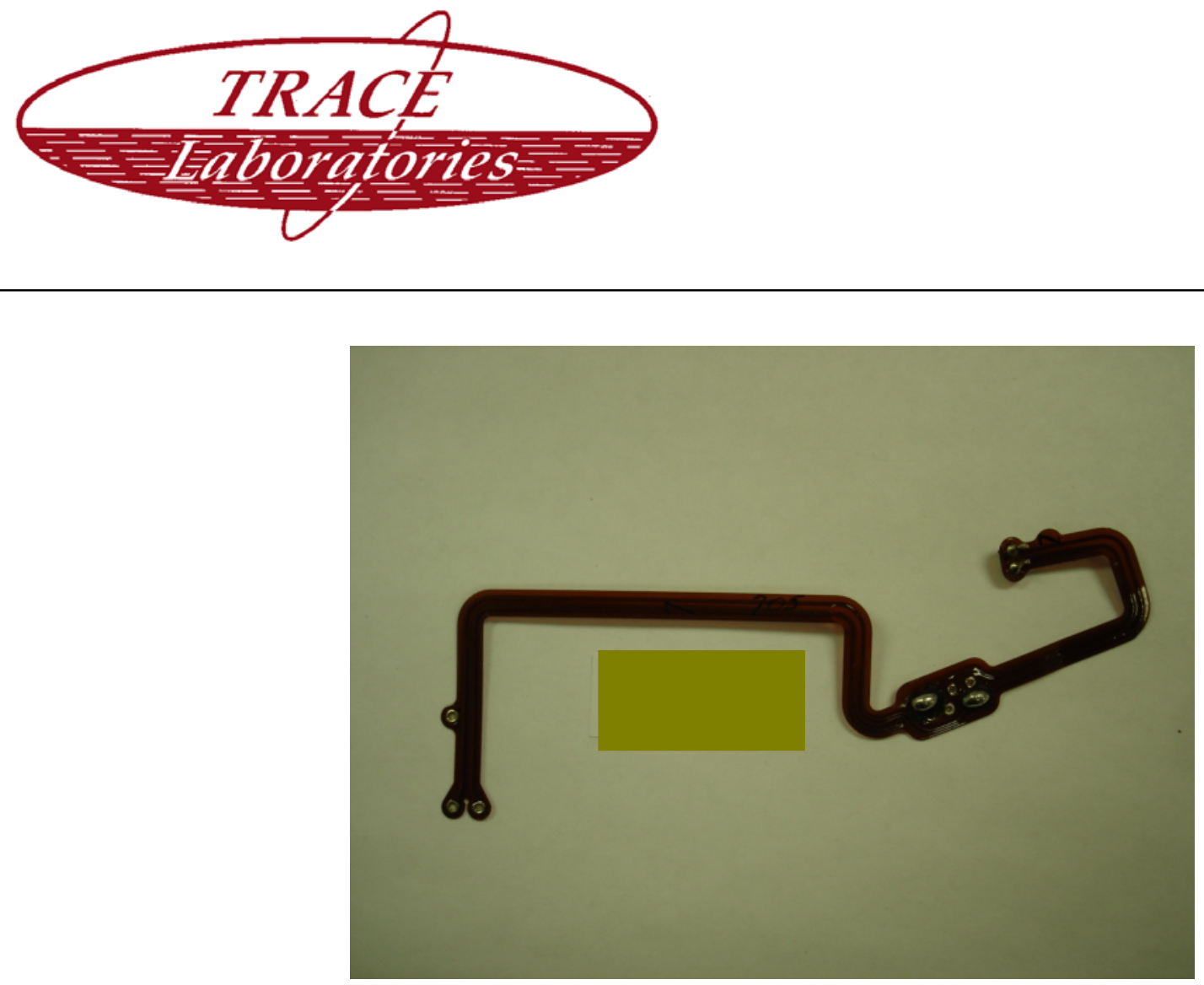

Photo 13:

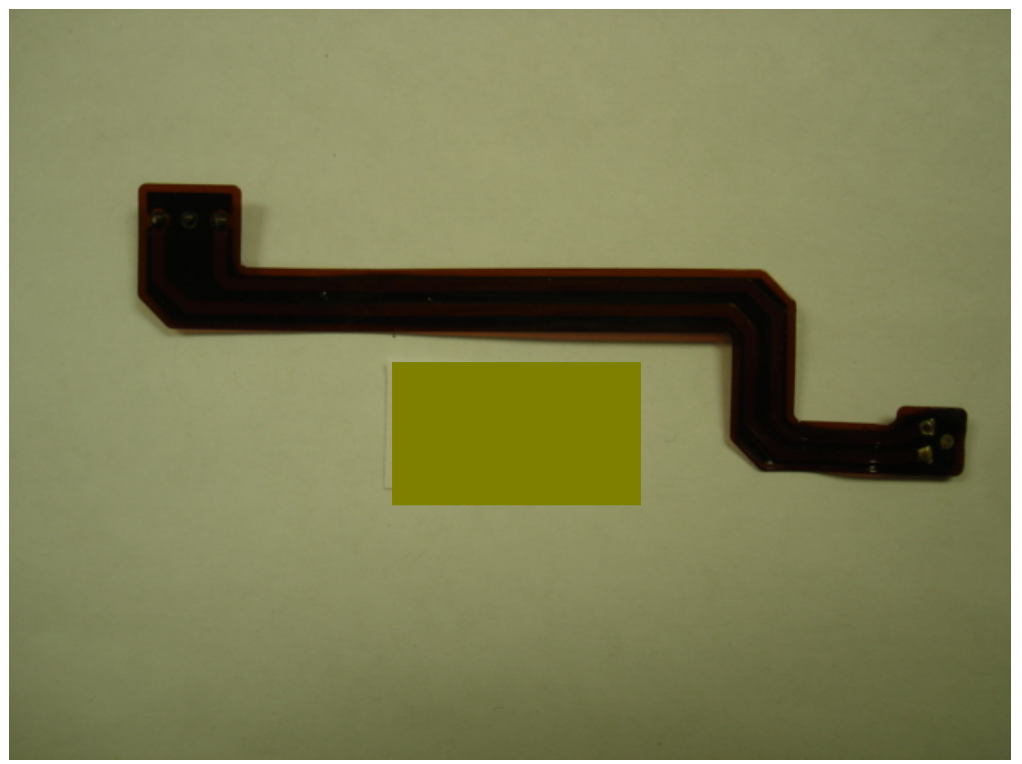

Photo 14:

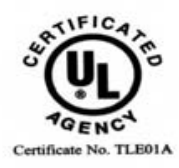

ISO 9001:2000

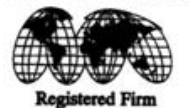

ISO/IEC 17025

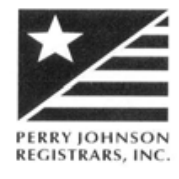

IPC

Registered Firm

S/O 66024 

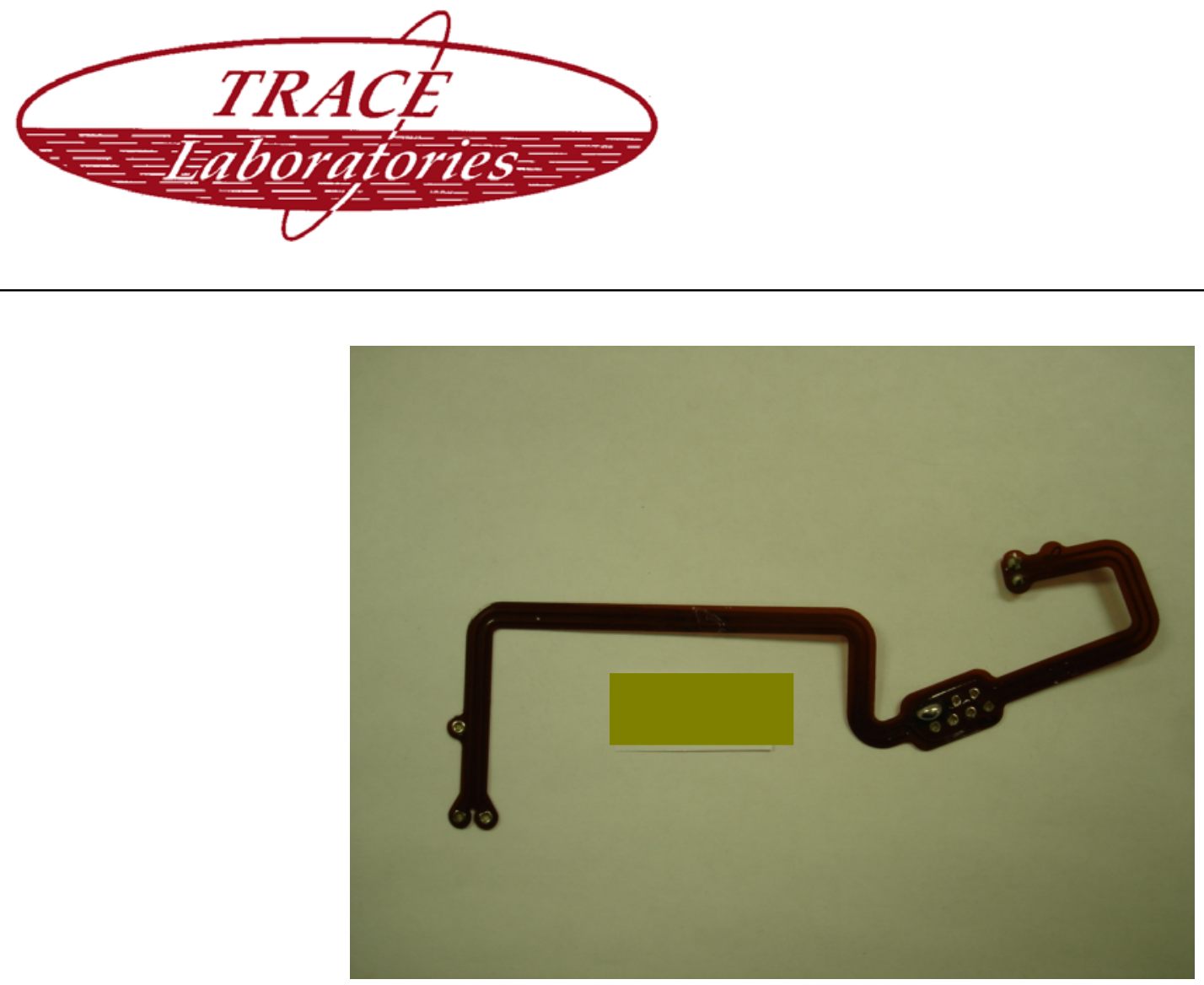

Photo 15:

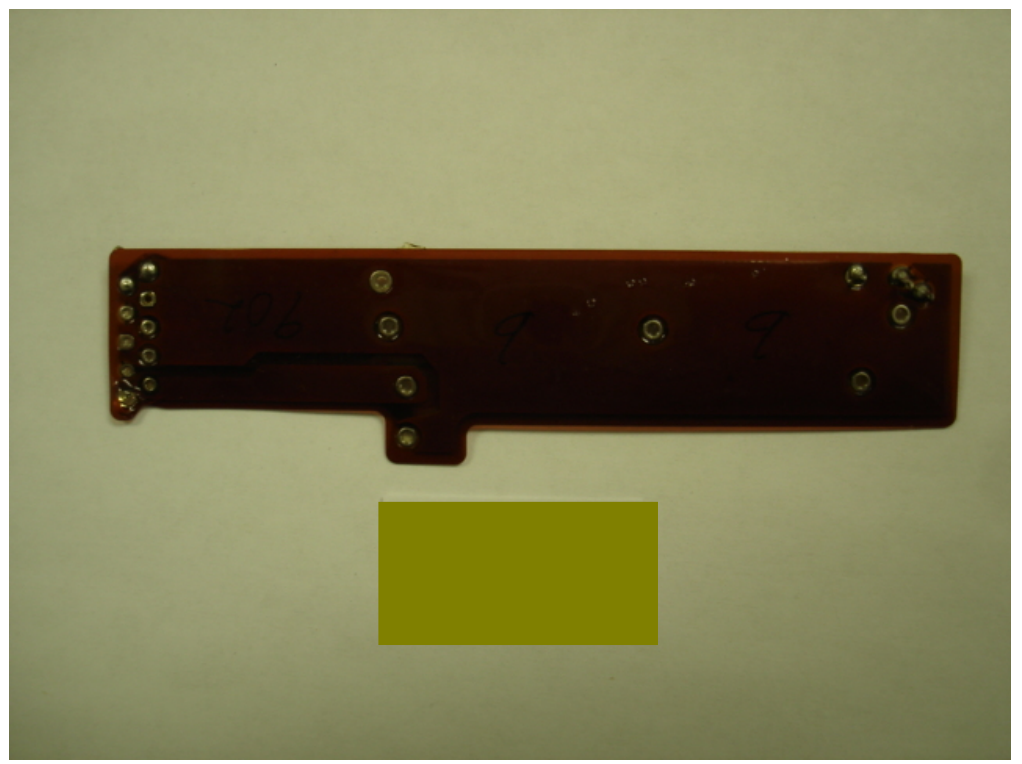

Photo 16:

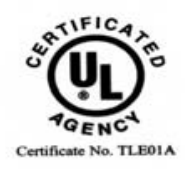

ISO 9001:2000

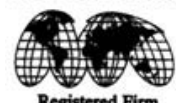

ISO/IEC 17025

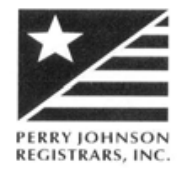

IPC

Registered Firm

S/O 66024 


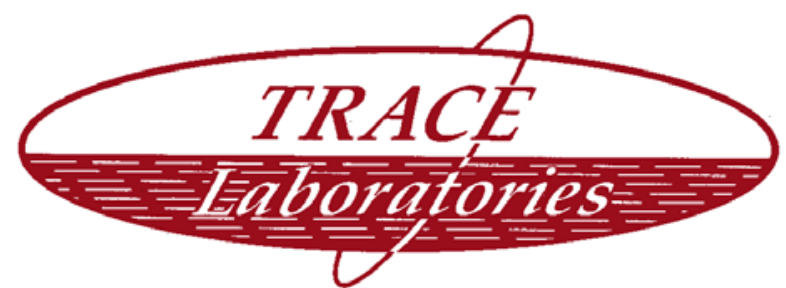

Trace Laboratories, Inc. certifies that the test equipment used complies with the calibration test purposes of ISO 10012-1, ANSI/NCSL Z540-1-1994, and MIL-STD-45662A and that the data contained in this report is accurate within the tolerance limitation of this equipment.

All test procedures detailed within this report are complete. The results in this report relate only to those items tested. If any additional information or clarification of this report is required, please contact us. This test report shall not be reproduced except in full, without the written approval of Trace Laboratories, Inc.

Thank you for selecting Trace Laboratories, Inc. for your testing requirements. Testing was subcontracted to an A2LA-approved laboratory.

\section{Lead Engineer:}

Senior Engineer
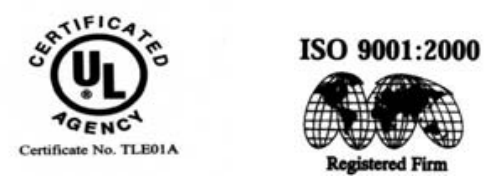


\section{TEST REPORT FOR: \\ HONEYWELL, FM\&T}

\section{DATE IN:}

Attn:

July 15, 2008

P/O \#:

\section{SUBMISSION IDENTIFICATION:}

Seven (7) groups of flexible printed circuit boards were submitted for Bending Test, MIR and Thermal Shock in accordance with IPC-6013A, Amendment 2, Class 3. The boards were identified as follows:

\begin{tabular}{|c|c|c|c|c|c|c|}
\hline Group 1 & Group 2 & Group 3 & Group 4 & Group 5 & Group 6 & Group 7 \\
\hline 1-1 & 2-1 & 3-1 & 4-1 & 5-1 & 6-1 & 7-1 \\
\hline $1-2$ & $2-2$ & $3-2$ & $4-2$ & $5-2$ & $6-2$ & $7-2$ \\
\hline $1-3$ & $2-3$ & $3-3$ & 4-3 & $5-3$ & $6-3$ & $7-3$ \\
\hline & & & & & & \\
\hline & & & & & & \\
\hline & & & & & & \\
\hline
\end{tabular}

SUMMARY:

The boards met the requirements of IPC-6013A, Amendment 2, Class 3, for Bending Test, MIR and Thermal Shock.

\section{APPROVED:}

Laboratory Director

SAMPLE DISPOSITION: Samples returned to the customer
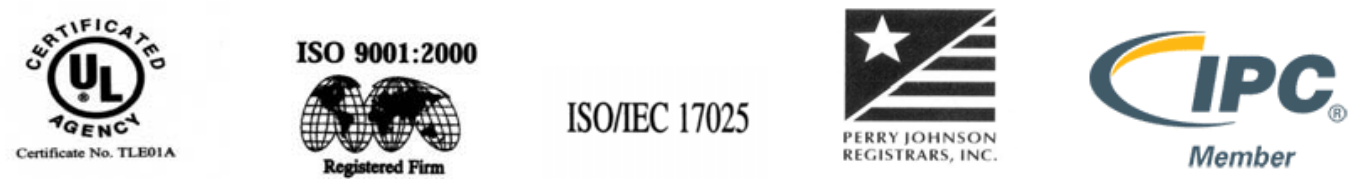

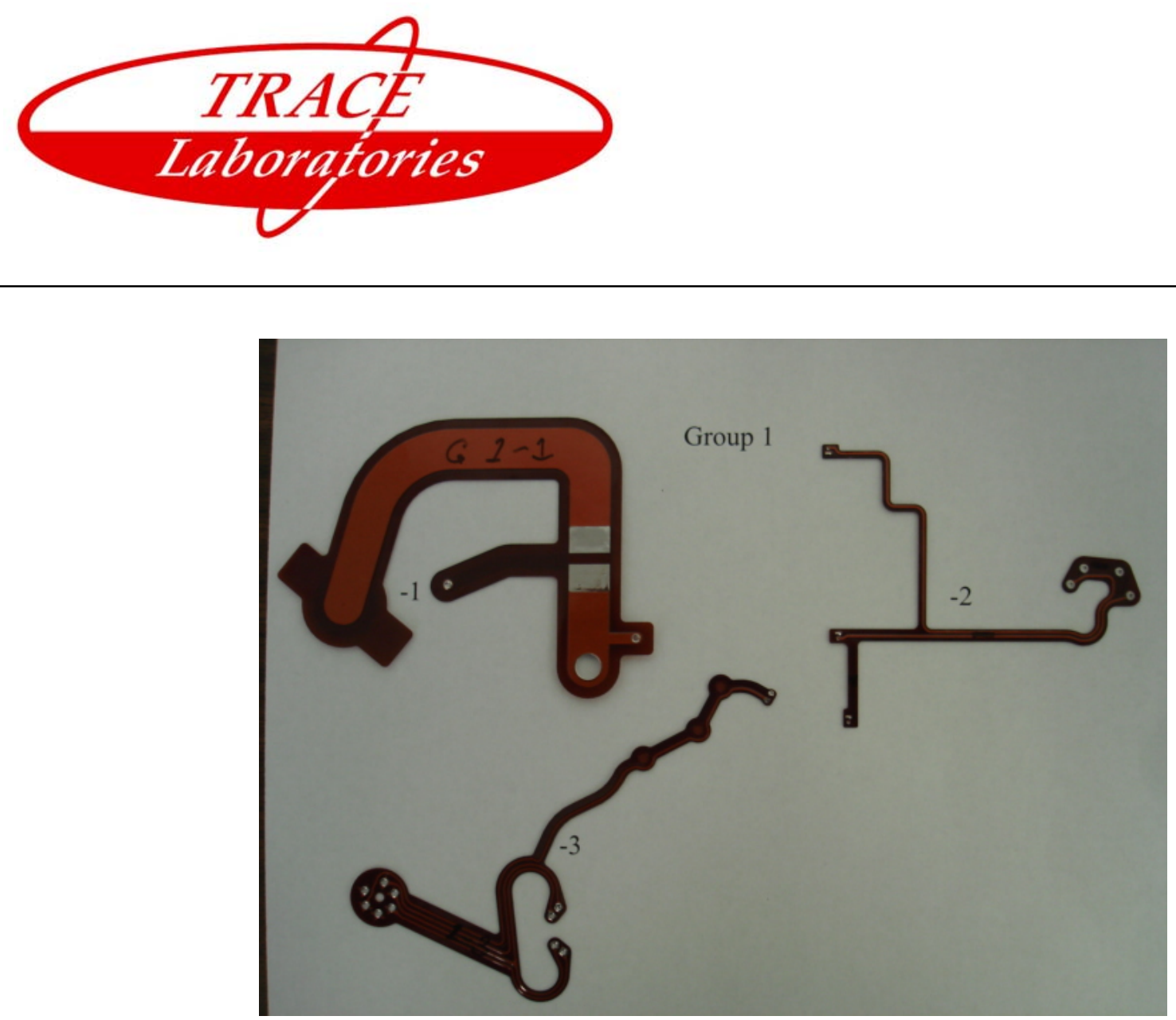

Photo 2: Group 1

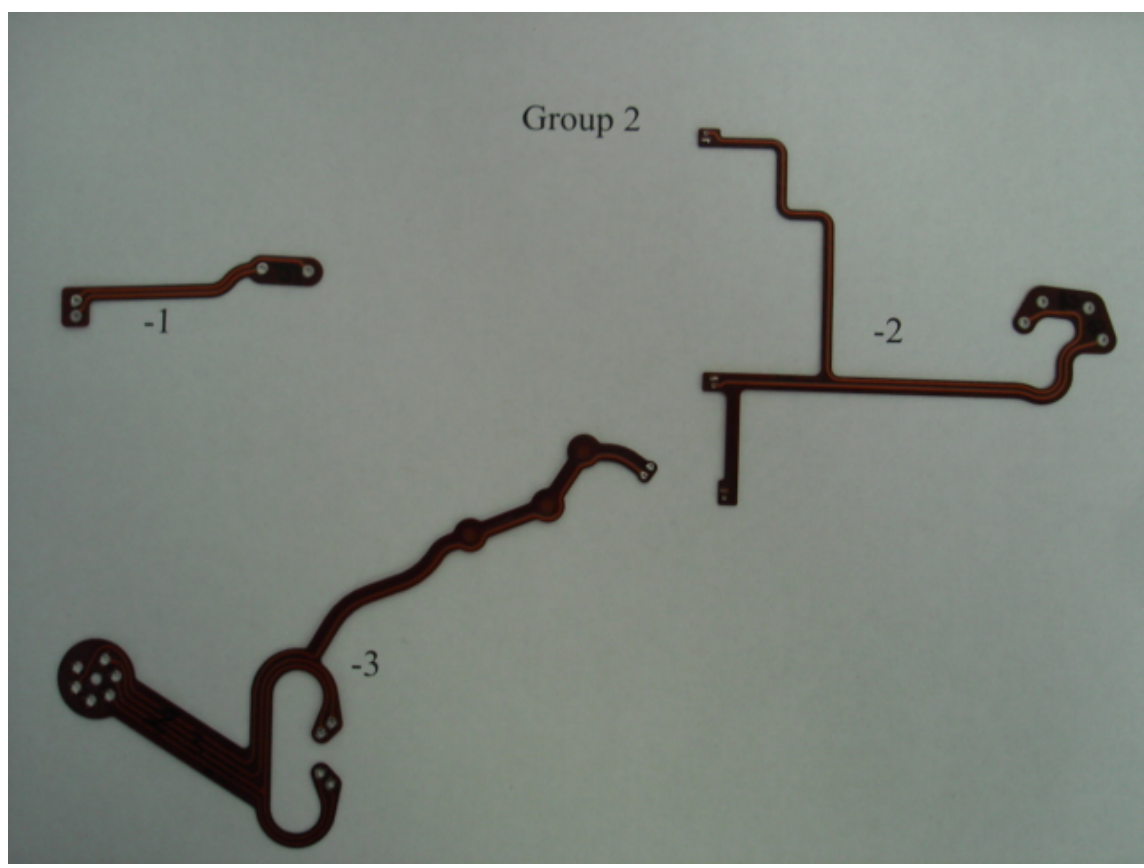

Photo 3: Group 2

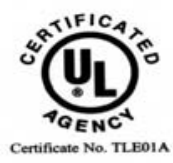

ISO 9001:2000

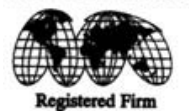

ISO/IEC 17025
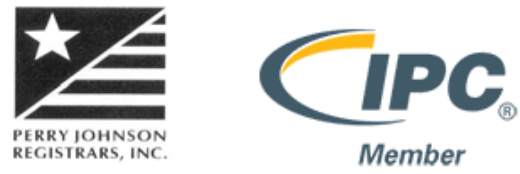

$\mathrm{S} / \mathrm{O} 69010$ 

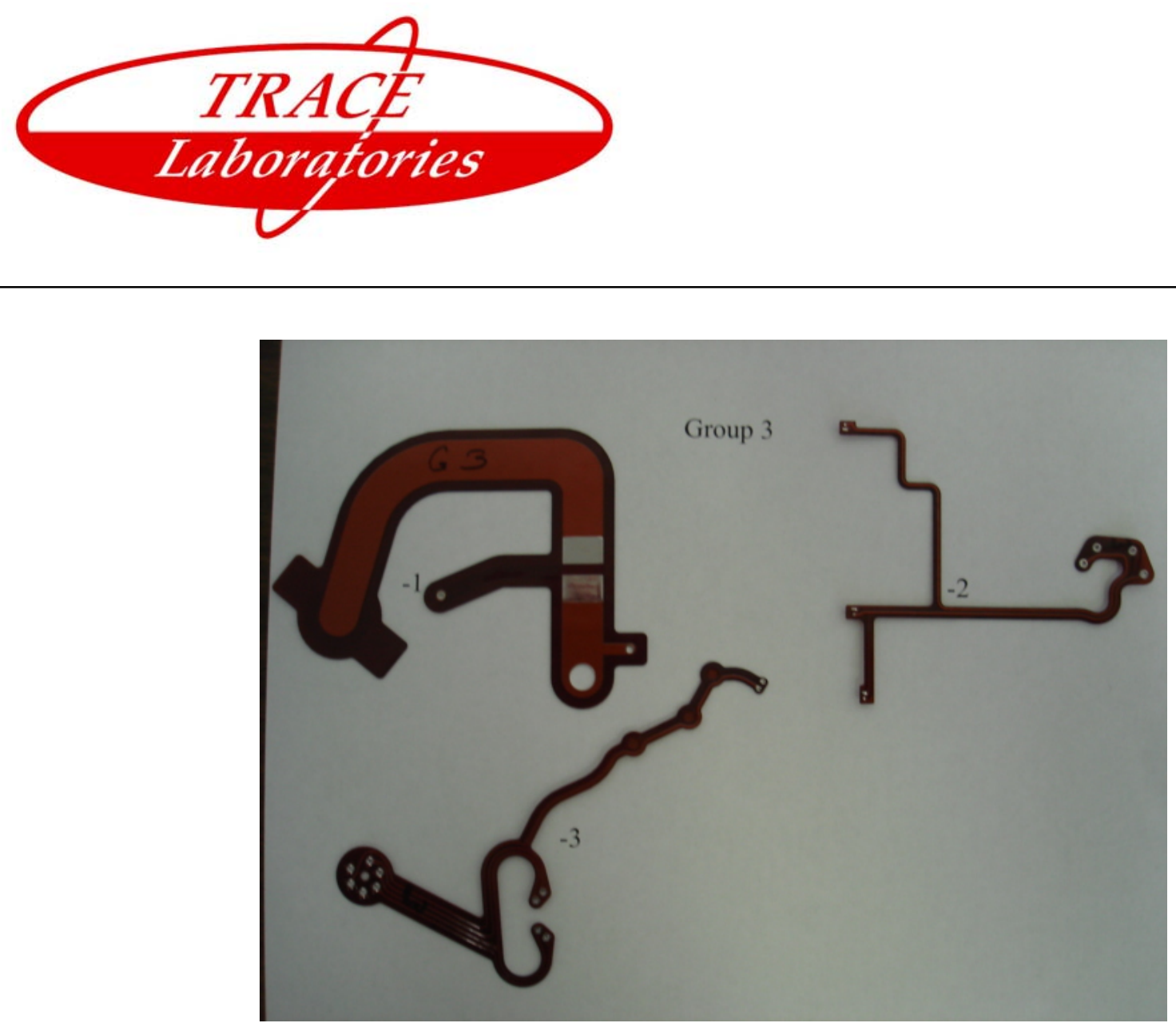

Photo 4: Group 3

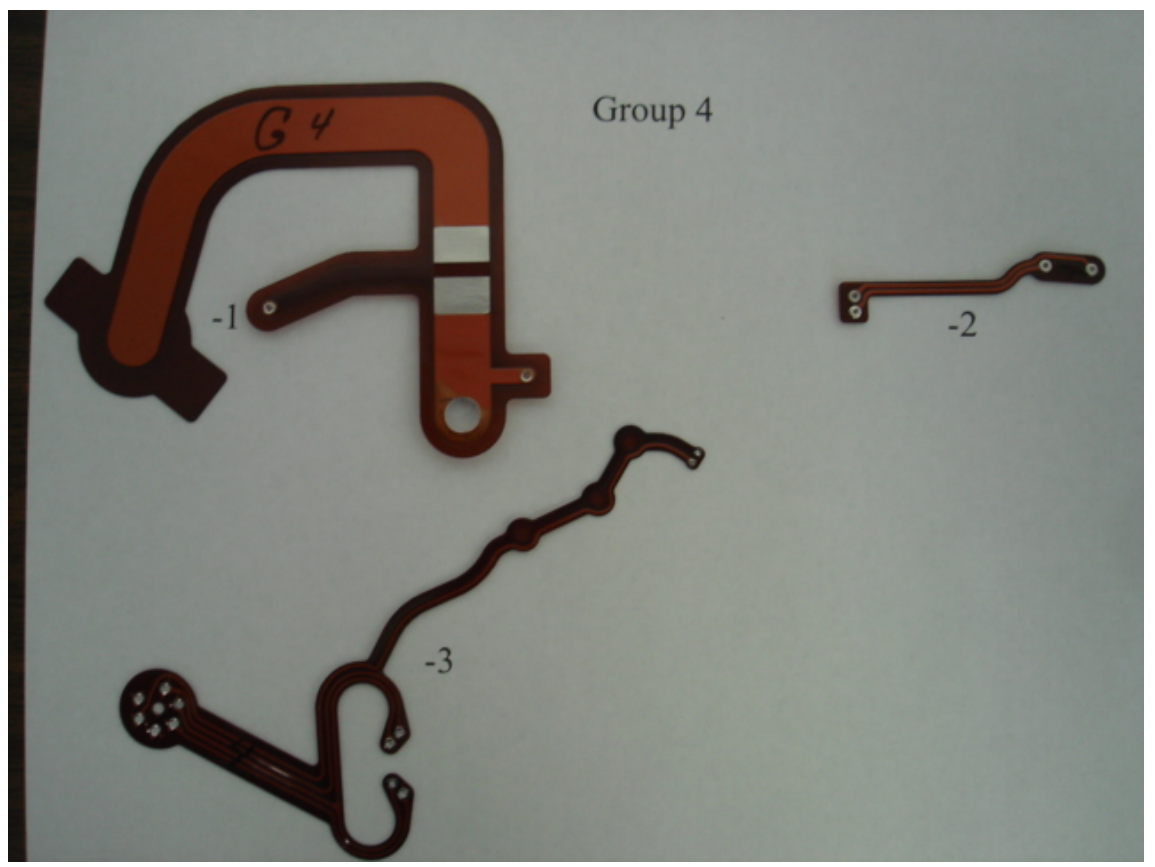

Photo 5: Group 4

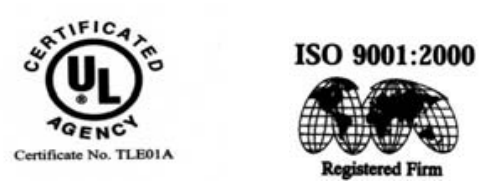

ISO/IEC 17025
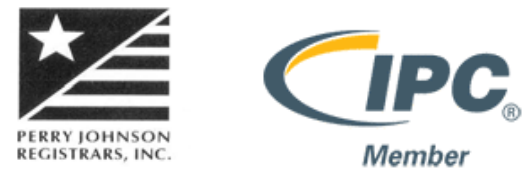

$\mathrm{S} / \mathrm{O} 69010$ 

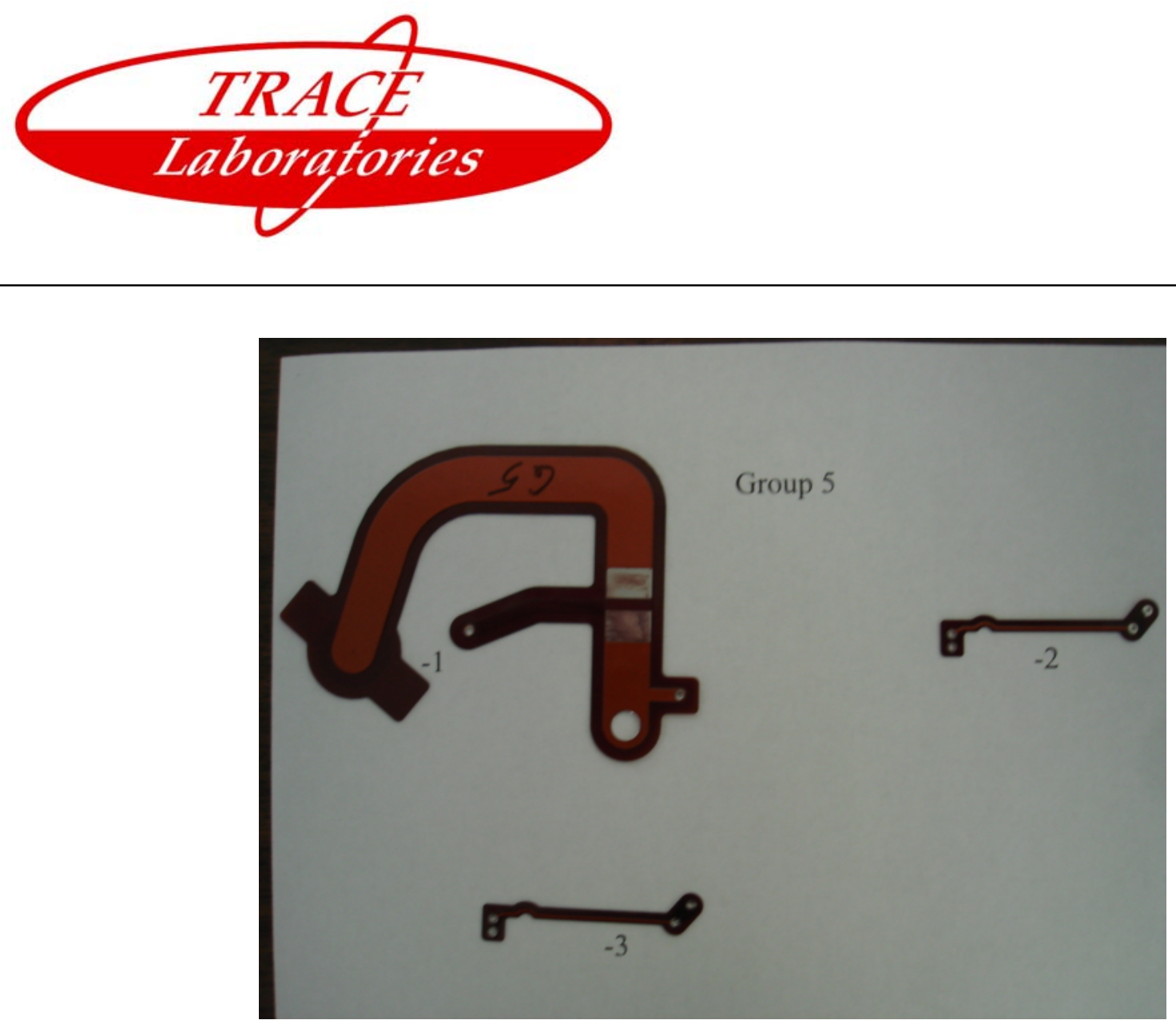

Photo 6: Group 5

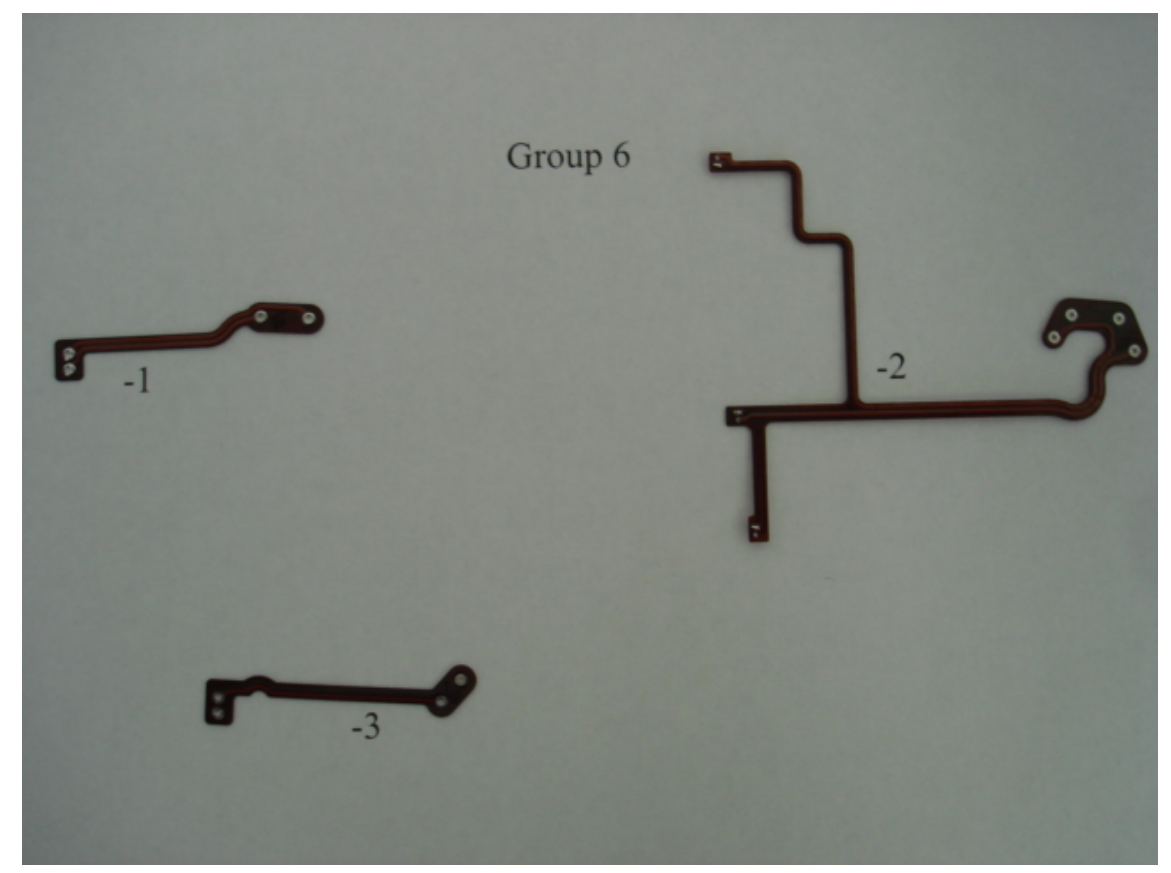

Photo 7: Group 6

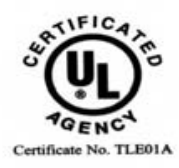

ISO 9001:2000

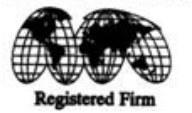

ISO/IEC 17025
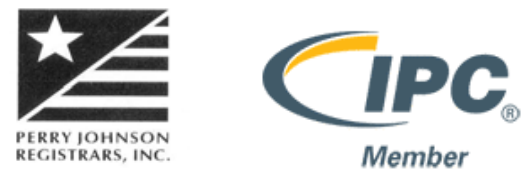

$\mathrm{S} / \mathrm{O} 69010$ 

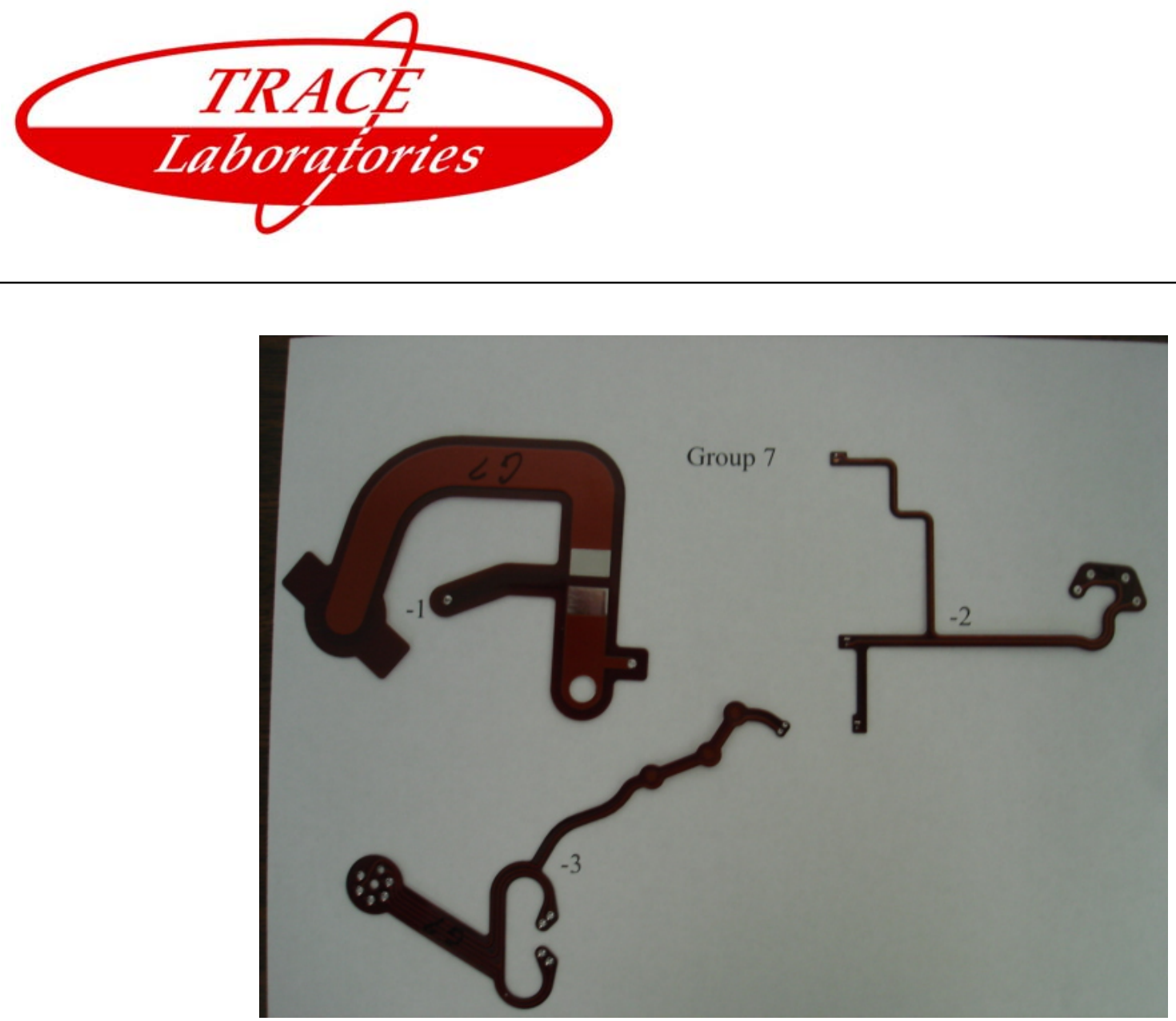

Photo 8: Group 7

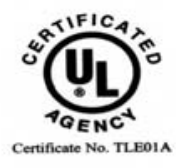

ISO 9001:2000

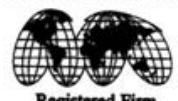

ISO/IEC 17025
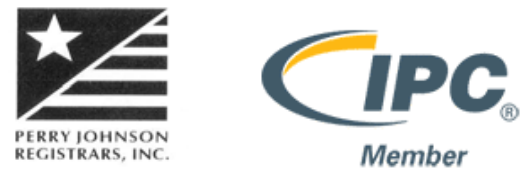

$\mathrm{S} / \mathrm{O} 69010$ 


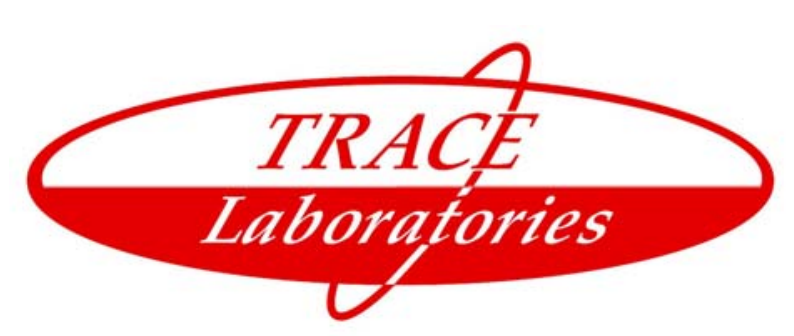

BENDING TEST

\section{TEST SPECIMENS:}

Seven (7) groups of flexible printed circuit boards.

\section{REFERENCE:}

IPC-6013A, Amendment 2, Class 3, paragraphs 3.6.1, 3.9 and 3.3.

\section{REQUIREMENT:}

After completion of the bending test in both directions, the flexible or rigid flexible printed wiring board shall be tested for electrical defects in accordance with 3.9 and shall meet the visual requirements of 3.3

\section{METHOD:}

The bending test shall conform to Figure 3-8, unless otherwise agreed to by the user. The bending test requirements shall be as specifies on the appropriate document / drawing. See IPC 2223 for guidance on the minimum bend radii. The following parameters shall be specified as minimum:

- Direction of bend (a).

- Degree of bend (b)

- Number of bend cycles (c)

- Diameter of mandrel (d)

- Points of application to be supplied by user.

A bend cycle is defined as taking one end of the specimen and bending it around a mandrel and then bending back to the original starting point, traveling $180^{\circ}$ in one direction and $180^{\circ}$ in the opposite direction. A bend cycle may also be defined as bending (using opposite ends) the ends towards each other (bend the same direction) and then bending them back to the original starting position, with each end traveling $90^{\circ}$ in the opposite direction.
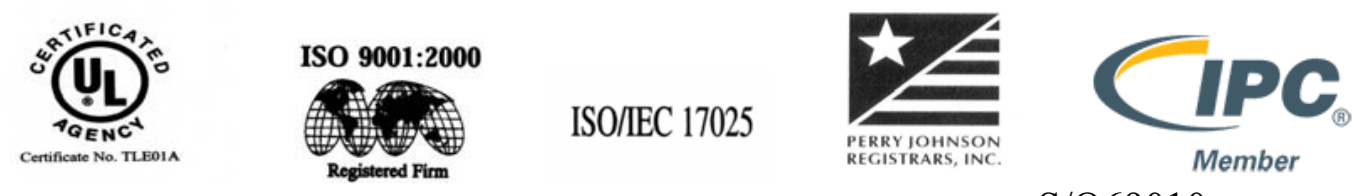

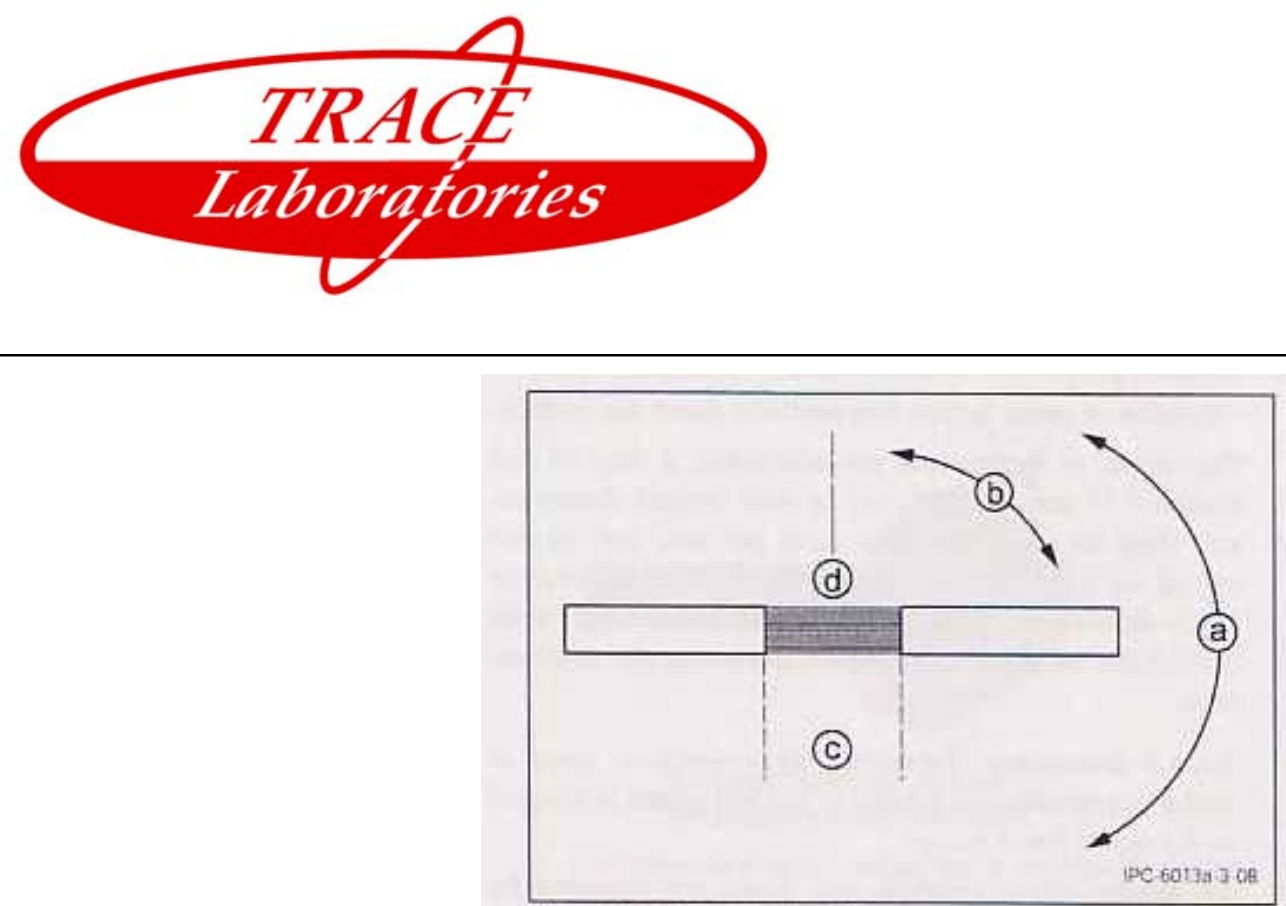

Figure 3-8 Bending Test

The direction of the bend was $90^{\circ}$ upward and $90^{\circ}$ downward.

The degree of the bends was $90^{\circ}$.

The number of bend cycles was 25 .

Mandrel Diameter:

\begin{tabular}{|c|c|c|}
\hline Groups & 10 times the thickness & Mandrel size \\
\hline 1 thru 7 & 160 mils & $.323^{\prime \prime}$ \\
\hline
\end{tabular}

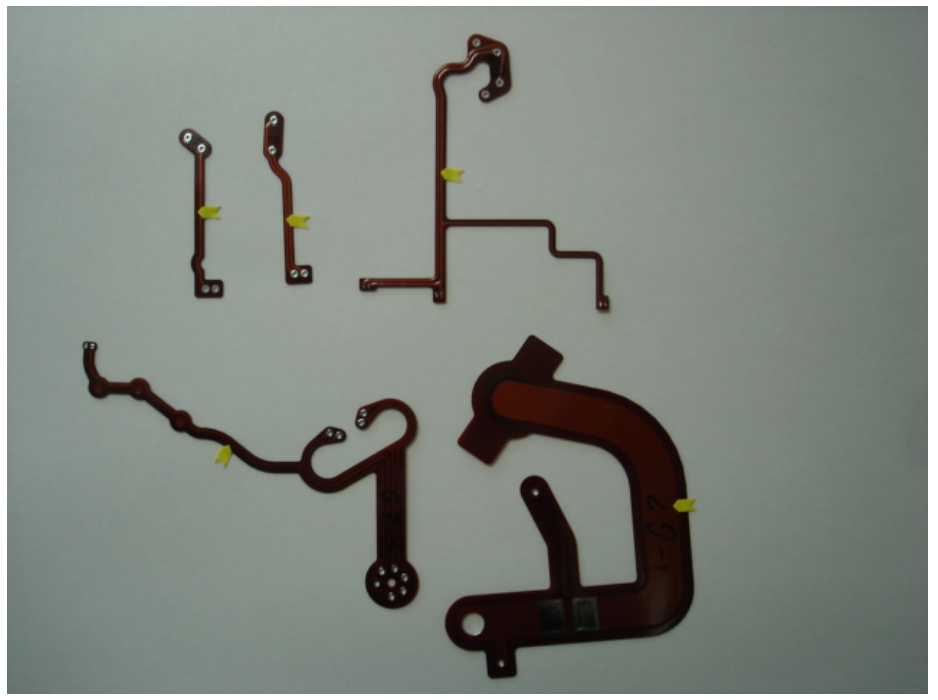

Photo 1: Bend Locations

After completing the bending test the boards were tested per electrical requirements 3.9.
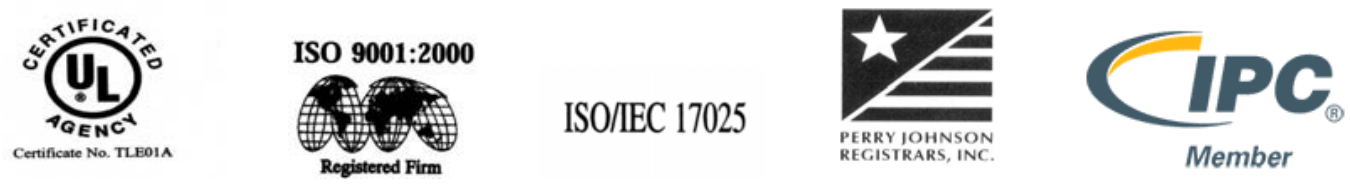


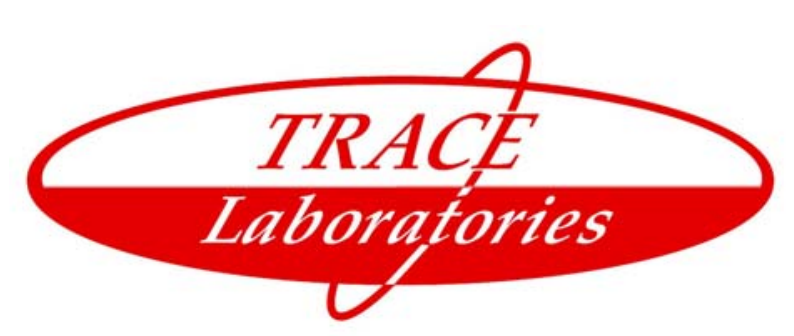

\section{ELECTRICAL REQUIREMENTS}

\section{CONTINUITY}

TEST SPECIMENS:

Seven (7) groups of flexible printed circuit boards.

\section{REFERENCE:}

IPC-6013A, Amendment 2, Class 3, paragraph 3.9.2.1.

\section{REQUIREMENT:}

Flexible printed boards and qualification testing of flexible printed wiring boards shall be tested in accordance with the procedure outlined below. There shall be no circuits whose resistance exceeds the values established in the procurement documentation. The presence of long runs of very narrow conductors, or high resistance metals may increase these values. When required by the user, interconnect shorts and continuity coupon $\mathrm{D}$ shall be used for evaluation of the interconnection resistance and circuit continuity.

A current shall be passed through each conductor or group of interconnected conductors by applying electrodes on the terminals at each end of the conductor or group of conductors. The current passing through the conductors shall not exceed that specified in IPC-2221 for the smallest conductor in the circuit. For qualification, the test current shall not exceed one ampere. Flexible printed wiring with designed resistive patterns shall meet the resistance requirements specified on the procurement documentation.

\section{METHOD:}

Insulated wires were soldered to the plated through holes at the ends of a selected conductor. The wires were placed in the grips of a multimeter. A resistance measurement was observed.

\section{RESULTS:}

The boards met the requirements for continuity between the conductor and land patterns.
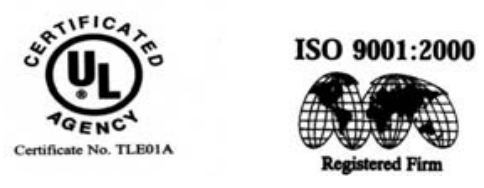

ISO/IEC 17025
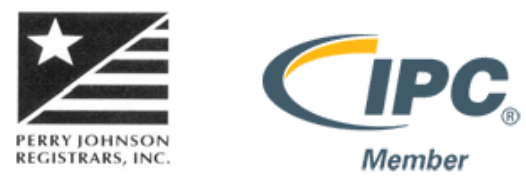


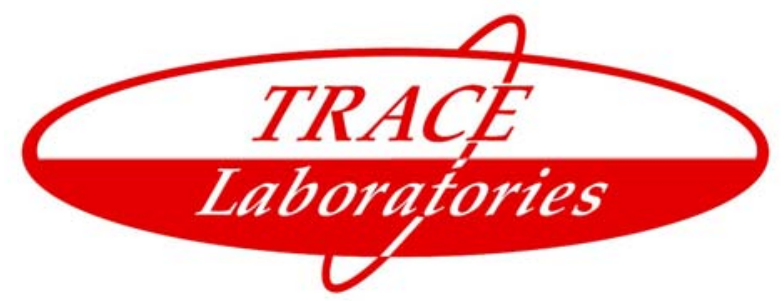

Group 1

\begin{tabular}{|c|c|c|c|c|}
\hline & Layer & $1-1$ & $1-2$ & $1-3$ \\
\hline Continuity & 1 & Pass & Pass & Pass \\
\hline & 2 & Pass & Pass & Pass \\
\hline Isolation & 1 & Pass & Pass & Pass \\
\hline & 2 & Pass & Pass & Pass \\
\hline
\end{tabular}

Group 2

\begin{tabular}{|c|c|c|c|c|}
\hline & Layer & $2-1$ & $2-2$ & $2-3$ \\
\hline Continuity & 1 & Pass & Pass & Pass \\
\hline & 2 & Pass & Pass & Pass \\
\hline Isolation & 1 & Pass & Pass & Pass \\
\hline & 2 & Pass & Pass & Pass \\
\hline
\end{tabular}

Group 3

\begin{tabular}{|c|c|c|c|c|}
\hline & Layer & $3-1$ & $3-2$ & $3-3$ \\
\hline Continuity & 1 & Pass & Pass & Pass \\
\hline & 2 & Pass & Pass & Pass \\
\hline Isolation & 1 & Pass & Pass & Pass \\
\hline & 2 & Pass & Pass & Pass \\
\hline
\end{tabular}

Group 4

\begin{tabular}{|c|c|c|c|c|}
\hline & Layer & $4-1$ & $4-2$ & $4-3$ \\
\hline Continuity & 1 & Pass & Pass & Pass \\
\hline & 2 & Pass & Pass & Pass \\
\hline Isolation & 1 & Pass & Pass & Pass \\
\hline & 2 & Pass & Pass & Pass \\
\hline
\end{tabular}
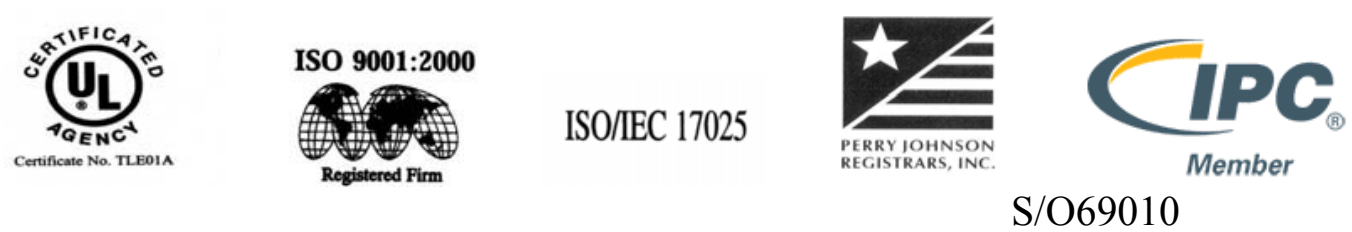


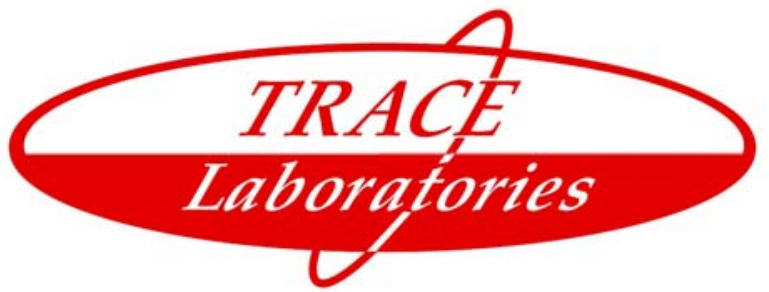

Group 5

\begin{tabular}{|c|c|c|c|c|}
\hline & Layer & $5-1$ & $5-2$ & $5-3$ \\
\hline Continuity & 1 & Pass & Pass & Pass \\
\hline & 2 & Pass & Pass & Pass \\
\hline Isolation & 1 & Pass & Pass & Pass \\
\hline & 2 & Pass & Pass & Pass \\
\hline
\end{tabular}

Group 6

\begin{tabular}{|c|c|c|c|c|}
\hline & Layer & $6-1$ & $6-2$ & $6-3$ \\
\hline Continuity & 1 & Pass & Pass & Pass \\
\hline & 2 & Pass & Pass & Pass \\
\hline Isolation & 1 & Pass & Pass & Pass \\
\hline & 2 & Pass & Pass & Pass \\
\hline
\end{tabular}

Group 7

\begin{tabular}{|c|c|c|c|c|}
\hline & Layer & $7-1$ & $7-2$ & $7-3$ \\
\hline Continuity & 1 & Pass & Pass & Pass \\
\hline & 2 & Pass & Pass & Pass \\
\hline Isolation & 1 & Pass & Pass & Pass \\
\hline & 2 & Pass & Pass & Pass \\
\hline
\end{tabular}
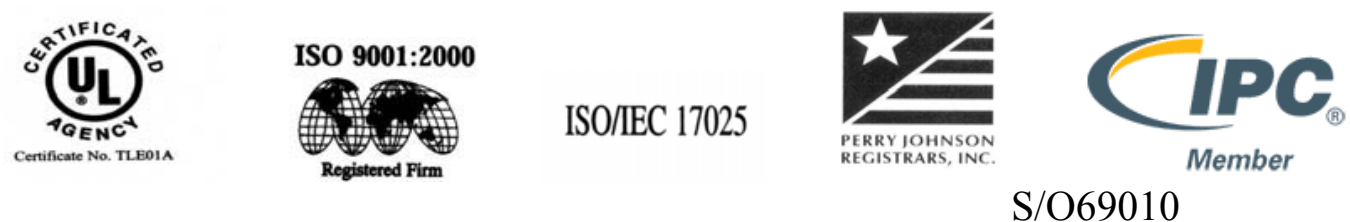


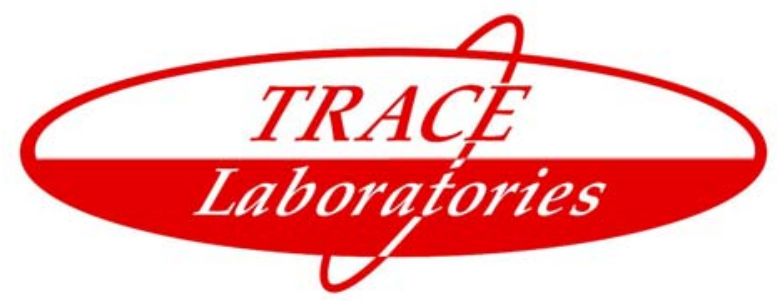

Insulation resistance measurements were made within two hours after removal from the chamber. Following 24 hours stabilization period, the test samples were visually examined.

Table 3-13 Insulation Resistance

\begin{tabular}{|c|c|c|}
\hline & Class 2 & Class 3 \\
\hline As Received & $500 \mathrm{M} \Omega$ & $500 \mathrm{M} \Omega$ \\
\hline After exposure to moisture & $100 \mathrm{M} \Omega$ & $500 \mathrm{M} \Omega$ \\
\hline
\end{tabular}

\section{RESULTS:}

\section{Visual Results:}

There was no evidence of mealing of the conformal coating.

Insulation Resistance Measurements (in megohms):

\begin{tabular}{|c|c|c|c|}
\hline Group 1 & Layer & Before Conditioning & After Conditioning \\
\hline-1 & 1 & $1.11 \times 10^{5}$ & $1.46 \times 10^{6}$ \\
\hline-1 & 1 & $5.16 \times 10^{5}$ & $3.20 \times 10^{6}$ \\
\hline-1 & 2 & $2.70 \times 10^{5}$ & $1.89 \times 10^{5}$ \\
\hline-2 & 1 & $6.98 \times 10^{6}$ & $1.82 \times 10^{6}$ \\
\hline-2 & 1 & $3.42 \times 10^{6}$ & $1.07 \times 10^{5}$ \\
\hline-2 & 2 & $3.96 \times 10^{6}$ & $1.62 \times 10^{6}$ \\
\hline-3 & 1 & $7.05 \times 10^{6}$ & $1.33 \times 10^{6}$ \\
\hline-3 & 1 & $8.87 \times 10^{6}$ & $1.64 \times 10^{6}$ \\
\hline
\end{tabular}

\begin{tabular}{|c|c|c|c|}
\hline Group 2 & Layer & Before Conditioning & After Conditioning \\
\hline-1 & 1 & $5.75 \times 10^{6}$ & $5.21 \times 10^{6}$ \\
\hline-2 & 1 & $6.27 \times 10^{5}$ & $3.22 \times 10^{6}$ \\
\hline-2 & 1 & $7.50 \times 10^{5}$ & $1.40 \times 10^{6}$ \\
\hline-2 & 2 & $1.11 \times 10^{5}$ & $1.18 \times 10^{6}$ \\
\hline-3 & 1 & $2.59 \times 10^{4}$ & $1.43 \times 10^{6}$ \\
\hline-3 & 1 & $7.54 \times 10^{4}$ & $1.13 \times 10^{5}$ \\
\hline
\end{tabular}
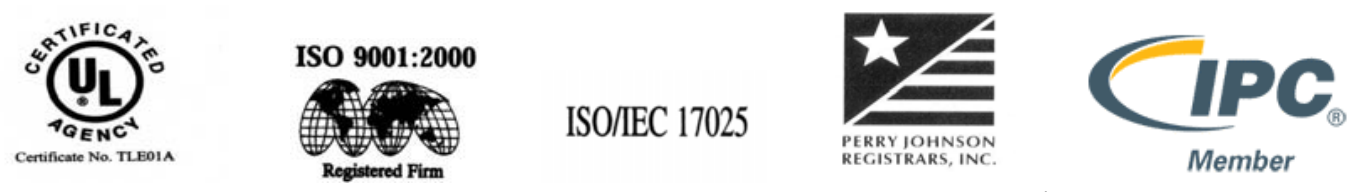


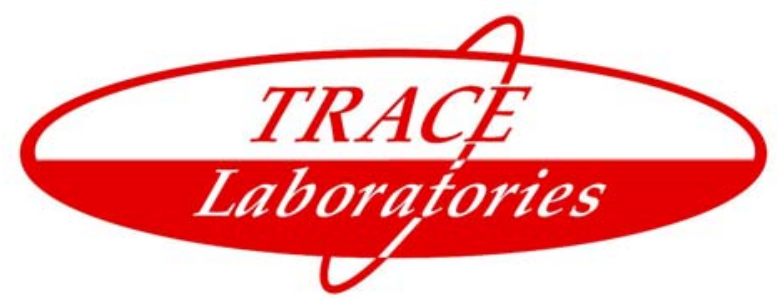

\begin{tabular}{|c|c|c|c|}
\hline Group 3 & Layer & Before Conditioning & After Conditioning \\
\hline-1 & 1 & $4.55 \times 10^{6}$ & $2.31 \times 10^{6}$ \\
\hline-1 & 1 & $5.02 \times 10^{5}$ & $1.16 \times 10^{6}$ \\
\hline-1 & 2 & $4.21 \times 10^{6}$ & $4.50 \times 10^{5}$ \\
\hline-2 & 1 & $2.83 \times 10^{6}$ & $1.19 \times 10^{6}$ \\
\hline-2 & 1 & $2.62 \times 10^{6}$ & $8.91 \times 10^{6}$ \\
\hline-2 & 2 & $2.38 \times 10^{6}$ & $8.57 \times 10^{5}$ \\
\hline-3 & 1 & $6.86 \times 10^{5}$ & $1.00 \times 10^{6}$ \\
\hline-3 & & $1.06 \times 10^{5}$ & \\
\hline
\end{tabular}

\begin{tabular}{|c|c|c|c|}
\hline Group 4 & Layer & Before Conditioning & After Conditioning \\
\hline-1 & 1 & $9.01 \times 10^{6}$ & $2.18 \times 10^{6}$ \\
\hline-1 & 1 & $4.29 \times 10^{6}$ & $8.98 \times 10^{5}$ \\
\hline-1 & 2 & $4.23 \times 10^{6}$ & $4.28 \times 10^{5}$ \\
\hline-2 & 1 & $4.52 \times 10^{6}$ & $4.35 \times 10^{6}$ \\
\hline-3 & 1 & $7.59 \times 10^{5}$ & $2.13 \times 10^{6}$ \\
\hline-3 & 1 & $7.72 \times 10^{5}$ & $1.28 \times 10^{6}$ \\
\hline
\end{tabular}

\begin{tabular}{|c|c|c|c|}
\hline Group 5 & Layer & Before Conditioning & After Conditioning \\
\hline-1 & 1 & $2.75 \times 10^{5}$ & $1.95 \times 10^{6}$ \\
\hline-1 & 1 & $4.88 \times 10^{5}$ & $2.57 \times 10^{6}$ \\
\hline-1 & 2 & $1.65 \times 10^{5}$ & $3.42 \times 10^{5}$ \\
\hline-2 & 1 & $2.76 \times 10^{6}$ & $2.62 \times 10^{6}$ \\
\hline-3 & 1 & $7.72 \times 10^{4}$ & $3.14 \times 10^{6}$ \\
\hline
\end{tabular}

\begin{tabular}{|c|c|c|c|}
\hline Group 6 & Layer & Before Conditioning & After Conditioning \\
\hline-1 & 1 & $4.73 \times 10^{6}$ & $2.65 \times 10^{6}$ \\
\hline-2 & 1 & $3.15 \times 10^{6}$ & $1.44 \times 10^{6}$ \\
\hline-2 & 1 & $2.94 \times 10^{6}$ & $8.93 \times 10^{5}$ \\
\hline-2 & 2 & $3.18 \times 10^{6}$ & $9.97 \times 10^{5}$ \\
\hline-3 & 1 & $4.01 \times 10^{6}$ & $2.50 \times 10^{6}$ \\
\hline
\end{tabular}
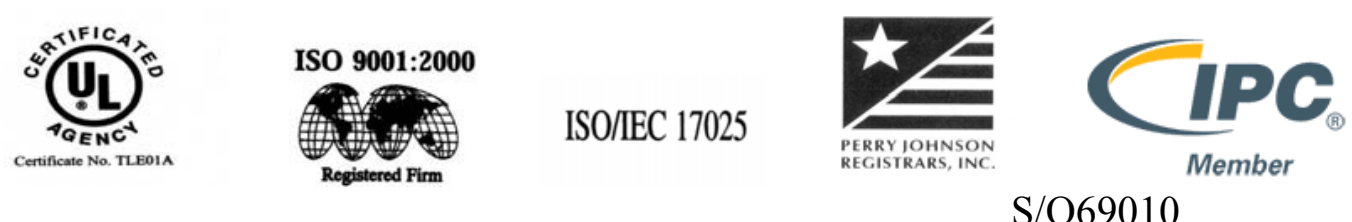


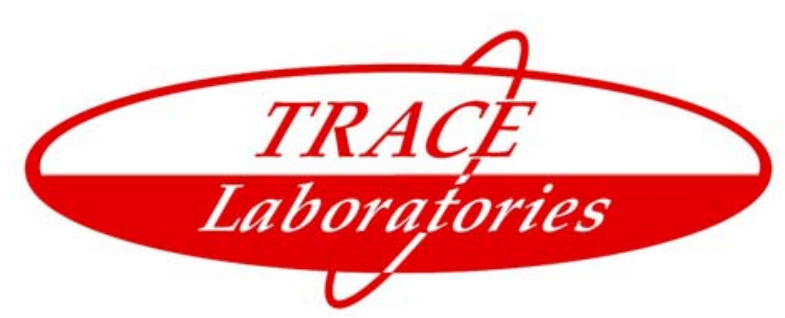

\section{METHOD:}

Insulated wires were soldered to a set of adjacent parallel conductors. The electrodes of the hi-pot tester were connected to the insulated wires. Five hundred VDC were applied for 30 seconds. The results were recorded.

\section{RESULTS:}

There was no flashover, or breakdown between conductor, or conductors and lands between all common portions of each conductor pattern and adjacent common portions of each conductor pattern.
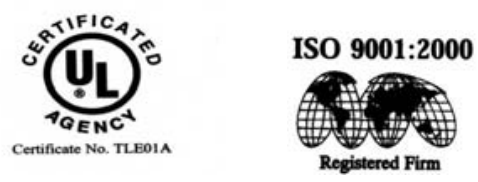


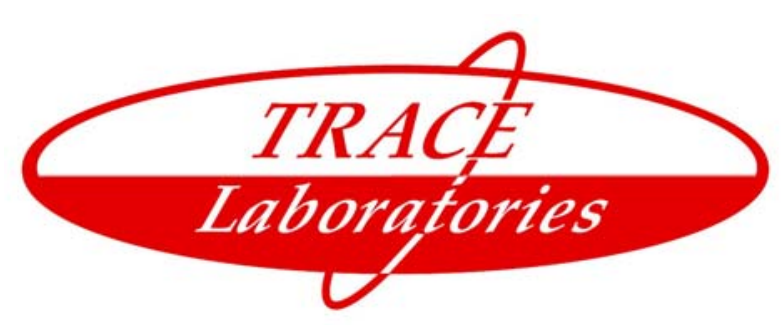

protruding dielectric materials, crazing, or haloing) shall not be cause for rejection. Imperfections in excess of that allowed shall be agreed upon between the fabricator and user, or as so stated on the procurement documentation.

3.3.2 Construction Imperfections Laminate imperfections include those characteristics that are both internal and external within the printed board but are visible from the surface.

3.3.2.1 Measling Measling is acceptable for all classes of end product, with the exception of highvoltage applications. Refer to IPC-A-600 for more information.

3.3.2.2 Crazing Crazing is acceptable for all classes of end product provided the imperfection does not reduce the conductor spacing below the minimum and there is no propagation of the imperfection as a result of thermal testing that replicates the manufacturing process. For Class 2 and 3, the distance of crazing shall not span more than $50 \%$ of the distance between adjacent conductors.

3.3.2.3 Delamination/ Blister Delamination and blistering is acceptable for all classes of end product provided the area affected imperfections does not exceed $1 \%$ of the board area on each side and does not reduce the spacing between conductive patterns below the minimum conductor spacing. There shall be no propagation of imperfections as a result of thermal testing that replicates the manufacturing process. For Class 2 and 3, the blister or delamination shall not span more than $25 \%$ of the distance between adjacent conductive patterns.

3.3.2.4 Foreign Inclusions Translucent particles trapped within the board shall be acceptable. Other particles trapped within the board shall be acceptable, provided the particle does not reduce the spacing between adjacent conductors to below the minimum spacing specified in 3.5.2.

3.3.2.5 Weave Exposure Weave exposure or exposed/disrupted fibers are acceptable for all Classes provided the imperfection does not reduce the remaining conductor spacing (excluding the area(s) with weave exposure) below the minimum. Refer to IPC-A-600 for more information.

3.3.2.6 Scratches, Dents, and Tool Marks Scratches, dents and tool marks are acceptable provided they do not expose conductors or expose/disrupt fibers greater than allowed in 3.3.2.4 and 3.3.2.5 and do not reduce the dielectric spacing below the minimum specified. Dents or tool marks that cause delamination, changes physical size of the conductor, or reduces condor width or spacing shall be rejected.

3.3.2.7 Surface Microvoids Surface microvoids are acceptable provided they do not exceed $0.8 \mathrm{~mm}$ [0.0315 in] in the longest dimension, bridge conductors, or exceed $5 \%$ of the total flexible printed wiring area.
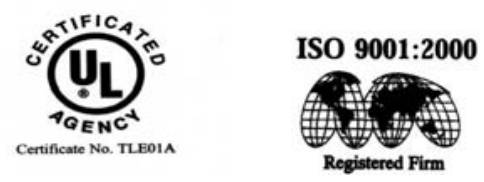

ISO/IEC 17025

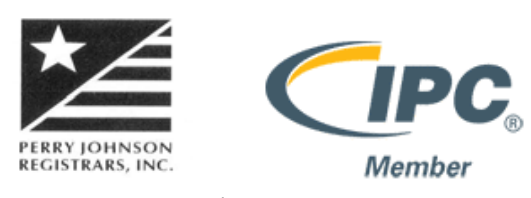

$\mathrm{S} / \mathrm{O} 69010$ 


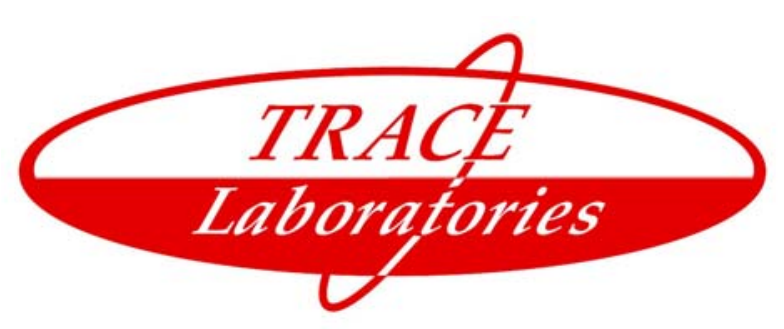

3.3.2.8 Color Variations in Bond Enhancement Treatment Mottled appearance or color variation in bond enhancement treatment is acceptable. Random missing areas of treatment shall not exceed $10 \%$ of the total conductor surface area of the affected layer.

3.3.2.9 Pink Ring There is no existing evidence that pink ring affects functionality. The presence of pink ring may be considered an indicator of process or design variation but is not a cause for rejection. The focus of concern should be the quality of the lamination bond.

3.3.2.10 Coverfilm Separations The coverfilm shall be uniform and free of coverfilm separations, such as wrinkles, creases, and soda strawing. Nonlamination shall be acceptable, provided such imperfections do not violate 3.3.2.4 and all of the following:

a. At random locations away from conductors, if each separation is no larger than $0.80 \mathrm{~mm} \mathrm{x}$ $0.80 \mathrm{~mm}$ [0.0315 in $\mathrm{x} 0.0315 \mathrm{in}]$ and is not within $1.0 \mathrm{~mm}$ [0.0394 in] of the board edge or the coverfilm opening. The total number of separations shall not exceed three in any $25 \mathrm{~mm}$ x $25 \mathrm{~mm}$ [0.984 in x $0.984 \mathrm{in}$ ] of coverfilm surface area.

b. The total separation shall not exceed $25 \%$ of the spacing between adjacent conductors.

c. There shall be no coverfilm nonlamination along the outer edges or covercoat openings of the coverfilm that reduces the seal below minimum edge to conductor spacing.

3.3.2.11.1 Covercoat Coverage Covercoat coverage manufacturing variations resulting in skips, voids, and misregistration are subject to the following restrictions:

a. Metal conductors shall not be exposed or bridged by blisters in areas where covercoat is required. Touch up, if required to cover these areas with covercoat, shall be of a material that is compatible and of equal resistance to soldering and cleaning as the originally applied covercoat.

b. In areas containing parallel conductors, covercoat variations shall not expose adjacent conductors unless the area between the conductors is purposely left blank as for a test point or for some surface mount devices.

c. Covercoat need not be flush with the surface of the land. Misregistration of a covercoatdefined feature shall not expose adjacent isolated lands or conductors.

d. Covercoat is allowed on lands for plated through holes to which solder connections are to be made, provided the external annular ring requirements for that class of products are not violated. Resist shall not encroach upon the barrel of this type of plated through holes.
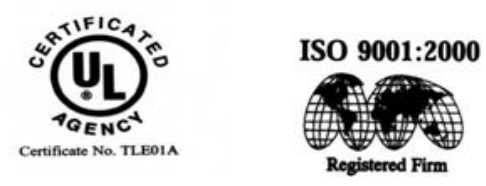

ISO/IEC 17025
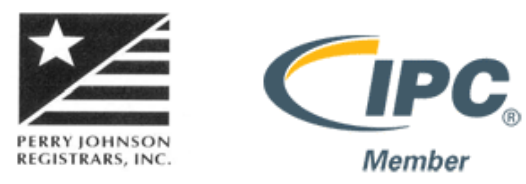

$\mathrm{S} / \mathrm{O} 69010$ 


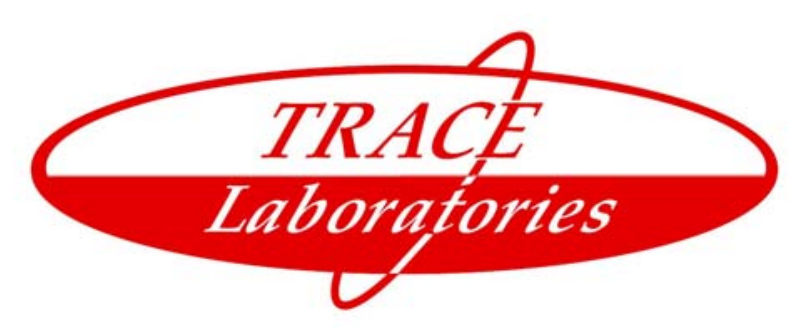

Other surfaces, such as edge flexible printed wiring connector fingers and surface mount lands, shall be free of covercoat, except as specified. Covercoat allowed in plated through holes and vias into which no component lead is soldered, unless the procurement documentation requires that the holes be completely solder filled. Covercoat may tent or plug via holes as specified by the procurement documentation. Test points that are intended for assembly testing must be free of covercoat unless coverage is specified.

e. When a land contains no plated through holes, as in the case of surface mount or ball grid array (BGA) lands, misregistration shall not cause encroachment of the covercoat on the land or lack of solder resist definition in excess of the following:

1) On surface mount lands, misregistration shall not cause encroachment of the covercoat over the land area greater than $0.050 \mathrm{~mm}$ [0.00197 in] for a pitch $\geq 1.25 \mathrm{~mm}$ [0.04921 in]. Encroachment shall not exceed $0.025 \mathrm{~mm}$ [0.00098 in] for a pitch $\leq 1.25 \mathrm{~mm}$ [0.04921 in] and encroachment may occur on adjacent sides, but not on opposite sides of a surface mount land.

2) On BGA lands, if the land is solder resist defined misregistration may allow a $90^{\circ}$ breakout of the covercoat on the land. If clearance is specified, no encroachment of the covercoat on the land is allowed, except at the conductor attachment.

3) On BGA lands connected to via holes, which have coverlayer dams required, the dam shall be continuous without missing peeling or cracked coverlayer, allowing a bare metal path between the BGA land and the via.

f. Blistering shall be allowed to the following extent:

1) Class 1: Does not bridge between conductors.

2) Class 2 and 3: Two per side, maximum $0.25 \mathrm{~mm}[0.00984 \mathrm{in}]$ in longest dimension, does not reduce electrical spacing between conductors by more than $25 \%$.

g. Pits and voids are allowed in nonconductor areas, provided they have adherent edges and do not exhibit lifting or blistering in excess of allowance in 3.3.2.11.1(f).

h. Coverage between closely spaced surface mount lands shall be as required by procurement documentation.
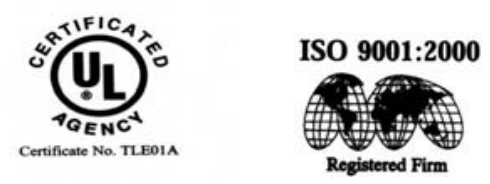

ISO/IEC 17025
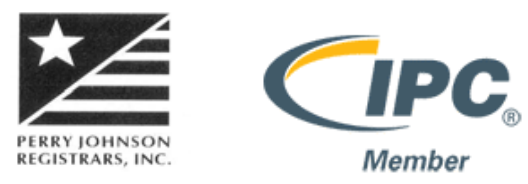

$\mathrm{S} / \mathrm{O} 69010$ 


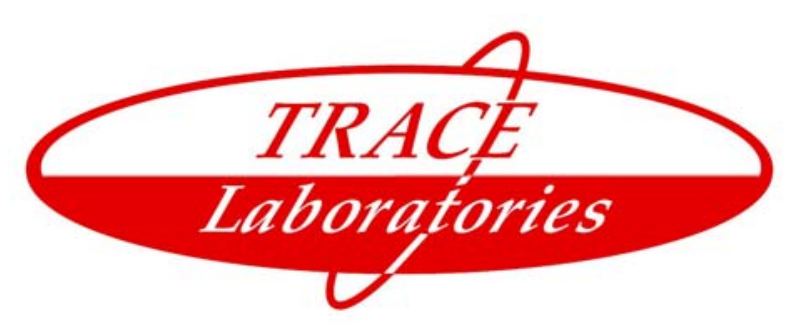

i. When design requires coverage to the flexible printed wiring edge, chipping or lifting of covercoat along the flexible printed wiring edge after fabrication shall not penetrate more than $1.25 \mathrm{~mm}$ [0.04291 in] or $50 \%$ of the distance to the closest conductor, whichever is less.

3.3.2.11.2 Covercoat Cure and Adhesion The cured covercoat shall not exhibit tackiness or blistering in excess of that permitted in 3.3.2.11.1(f). When tested in accordance with IPC-TM-650, Method 2.4.28.1, the maximum percentage of cured covercoat lifting from Coupon G identified in IPC-2221 shall be in accordance with Table 3-1.

Table 3-1 Covercoat Adhesion

\begin{tabular}{|l|c|c|c|}
\hline \multirow{2}{*}{ Surface } & \multicolumn{3}{|c|}{ Maximum Percentage Loss Allowed } \\
\cline { 2 - 4 } & Class 1 & Class 2 & Class 3 \\
\hline Bare Copper & 10 & 5 & 0 \\
\hline Gold Nickel & 25 & 10 & 5 \\
\hline Base Laminate & 10 & 5 & 0 \\
\hline $\begin{array}{l}\text { Melting Metals } \\
\text { (Tin-lead, fused tin- } \\
\text { lead, and bright } \\
\text { acid-tin) }\end{array}$ & 50 & 25 & 10 \\
\hline
\end{tabular}

3.3.2.11.3 Covercoat Thickness Covercoat thickness is not measured, unless specified on the procurement documentation. If a thickness measurement is required, instrumental methods may be used or an assessment may be made using a microsection of the parallel conductors on Coupon E identified in IPC-2221.

3.3.2.12 Solder Wicking/Plating Penetration Solder wicking or other plating penetration shall not extend into a bend or flex transition area and shall meet the conductor spacing requirements. Solder wicking or other plating penetration shall not exceed the limits specified in Table 3-2.

Table 3-2 Solder Wicking/Plating Penetration Limits

\begin{tabular}{|c|c|c|}
\hline Class 1 & Class 2 & Class 3 \\
\hline $\begin{array}{c}\text { As agreed upon between } \\
\text { user and supplier }\end{array}$ & $0.5 \mathrm{~mm}\left(0.020^{\prime \prime}\right)$ maximum & $0.3 \mathrm{~mm}(0.012$ "') maximum \\
\hline
\end{tabular}

3.3.2.13 Stiffener A stiffener will be evaluated only as a mechanical support. Void free bonding of the stiffener to the flexible printed wiring is not required. Specific requirements shall be as agreed upon between user and supplier.
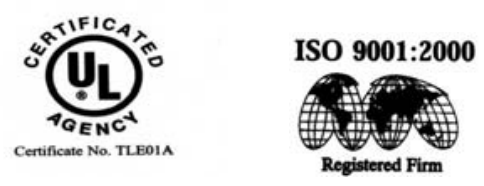

ISO/IEC 17025
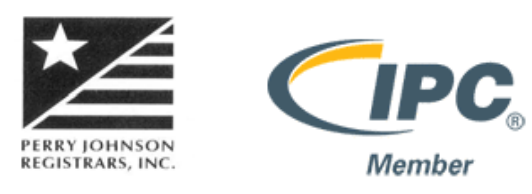

$\mathrm{S} / \mathrm{O} 69010$ 


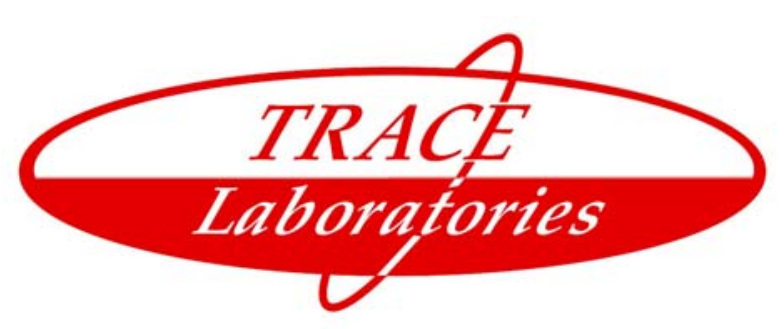

3.3.1 Plating and Coating Voids in the Hole Plating and coating voids shall not exceed that allowed by Table 3-3.

Table 3-3 Plating and Coating Voids Visual Examination

\begin{tabular}{|l|l|l|l|}
\hline \multicolumn{1}{|c|}{ Material } & \multicolumn{1}{c|}{ Class 1 } & \multicolumn{1}{c|}{ Class 2 } & \multicolumn{1}{c|}{ Class 3 } \\
\hline Copper $^{1}$ & $\begin{array}{l}\text { Three voids allowed } \\
\text { per hole in not more } \\
\text { than } 10 \% \text { of the holes. }\end{array}$ & $\begin{array}{l}\text { One void allowed per } \\
\text { hole in not more than } \\
5 \% \text { of the holes. }\end{array}$ & None \\
\hline Finish Coating $^{2}$ & $\begin{array}{l}\text { Five voids allowed per } \\
\text { hole in not more than } \\
15 \% \text { of the holes. }\end{array}$ & $\begin{array}{l}\text { Three voids allowed } \\
\text { per hole in not more } \\
\text { than 5\% of the holes. }\end{array}$ & $\begin{array}{l}\text { One void allowed per } \\
\text { hole in not more than } \\
5 \% \text { of the holes. }\end{array}$ \\
\hline
\end{tabular}

${ }^{1}$ For class 2 flexible printed wiring product, copper voids shall not exceed $5 \%$ of the hole length. For class 1 flexible printed wiring product, copper voids shall not exceed $10 \%$ of the hole length. Circumferential voids shall not extend beyond $90^{\circ}$ of the circumference.

${ }^{2}$ For class 2 and 3 product, finished coating voids shall not exceed $5 \%$ of the hole length. For class 1, finished coating voids shall not exceed $10 \%$ of the hole length. Circumferential voids shall not extend beyond $90^{\circ}$ for class 1,2 , or 3 .

3.3.4 Markings If required, each individual flexible printed board, qualification flexible printed board, and set of quality conformance test circuit strips (as opposed to each individual test coupon) shall be marked. This marking is required in order to insure traceability between the flexible printed wiring/test strips and the manufacturing history and to identify the supplier (logo, etc.). If size or space does not permit marking individual flexible printed wiring, bagging or tagging is permitted.

The marking shall be produced by the same process as used in producing the conductive pattern, or by use of a permanent fungistatic ink or paint (see 3.2.10), LASER marker or by vibrating pencil marking on a metallic area provided for marking purposes.

Conductive markings, either etched copper or conductive ink (see 3.2.10) shall be considered as electrical elements of the circuit and shall not reduce the electrical spacing requirements. All markings shall be compatible with materials and parts, legible for all tests, and in no case affect flexible printed wiring performance.

Marking shall not cover areas of lands that are to be soldered. (see IPC-A-600 for legibility requirements). In addition to this marking, the use of bar code marking is permissible. When used, date code shall be formatted per the supplier's discretion in order to establish traceability as to when the manufacturing operations were performed.

3.3.5 Solderability Only those flexible printed boards that require soldering in a subsequent assembly operation require solderability testing. Solderability testing is not necessary for flexible printed wiring that does not require soldering. This shall be specified on the master drawing, as in the case where press-
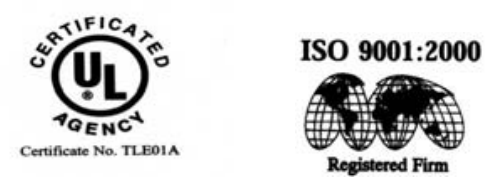

ISO/IEC 17025
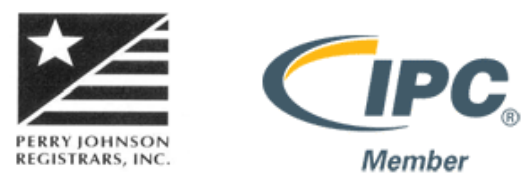

$\mathrm{S} / \mathrm{O} 69010$ 


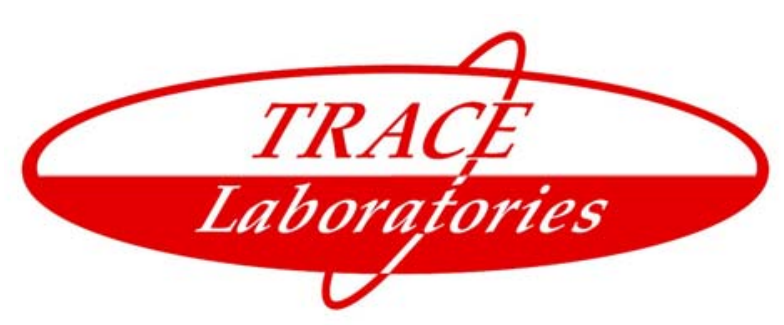

fit components are used. Flexible printed wiring to be used for surface mount does not require hole solderability testing.

When required by the procurement documentation, accelerated aging for coating durability shall be in accordance with J-STD-003. The Category of durability shall be specified on the master drawing; however, in not specified, Category 2 shall be used. Specimens to be tested shall be conditioned, if required, and evaluated for surface and hole solderability using J-STD-003.

When solderability testing is required, consideration should be given to flexible printed wiring thickness and copper thickness. As both increase, the amount of time to properly wet the sides of the holes and the tops of the lands increases proportionately.

Note: Accelerated aging (steam aging) is intended for use on coatings of tin/lead solder or tin, but not other final finishes.

3.3.6 Plating Adhesion The adhesion of the plating shall be tested in accordance with IPC-TM-650, method 2.4.1. There shall be no evidence of any portion of the protective plating or the conductor pattern foil being removed, as shown by particles of the plating or pattern foil adhering to the tape. If overhanging metal (slivers) breaks off and adheres to the tape, it is evidence of overhang or slivers, but not of plating adhesion failure.

3.3.7 Edge Board Contact, Junction of Gold Plate to Solder Finish Exposed copper/plating overlap between the solder finish and gold plate shall meet the requirements of Table 3-4. The exposed copper /plating or gold overlap may exhibit a discolored or gray-black area which is acceptable (see 3.5.3.3).

Table 3-4 Edge Board Contact Gap

\begin{tabular}{|c|c|c|}
\hline Class & Maximum Exposed Copper Gap & Maximum Gold Overlap \\
\hline 1 & $2.5 \mathrm{~mm}[0.0984 \mathrm{in}]$ & $2.5 \mathrm{~mm}[0.0984 \mathrm{in}]$ \\
\hline 2 & $1.25 \mathrm{~mm}[0.04921 \mathrm{in}]$ & $1.25 \mathrm{~mm}[0.04921 \mathrm{in}]$ \\
\hline 3 & $0.8 \mathrm{~mm}[0.031 \mathrm{in}]$ & $0.8 \mathrm{~mm}[0.031 \mathrm{in}]$ \\
\hline
\end{tabular}

3.3.8 Lifted Lands When visually examined in accordance with 3.3, there shall be no lifted lands on the delivered (nonstressed) printed circuit board.
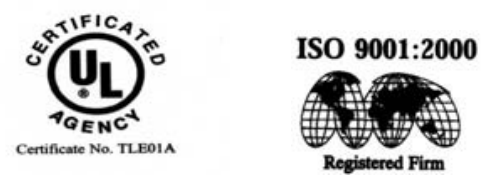

ISO/IEC 17025
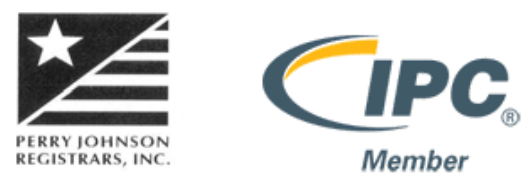

$\mathrm{S} / \mathrm{O} 69010$ 


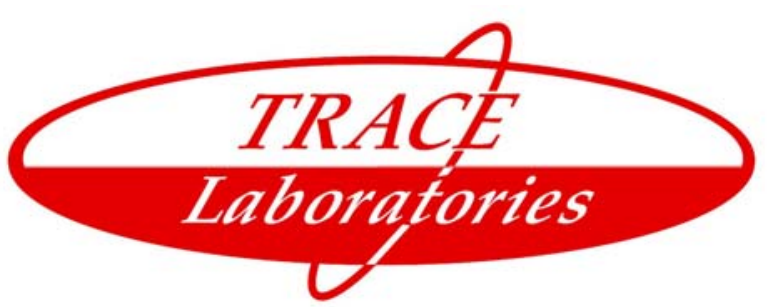

3.3.9 $\mathrm{W}$ orkmanship Flexible printed boards shall be processed in such a manner as to be uniform in quality and show no visual evidence of dirt, foreign matter, oil, fingerprints, tin/lead or solder smear transfer to the dielectric surface, flux residue and other contaminants that affect life, ability to assemble and serviceability. Visually dark appearances in non-plated holes, which are seen when a metallic or nonmetallic semiconductive coating is used, are not foreign material and do not affect life or function. Flexible printed wiring shall be free of defects in excess of those allowed in this specification. There shall be no evidence of any lifting or separation of platings from the surface of the conductive pattern, or of the conductor from the base laminate in excess of that allowed. There shall be no loose plating slivers on the surface of the printed board.

\section{METHOD:}

The boards were visually examined with approximately $1.75 \mathrm{X}$ using various light sources. Progressively higher magnifications, up to $40 \mathrm{X}$, were used for referee evaluation.

\section{RESULTS:}

The boards met the requirements of IPC-6013A, Amendment 2, Class 3, paragraph 3.3.
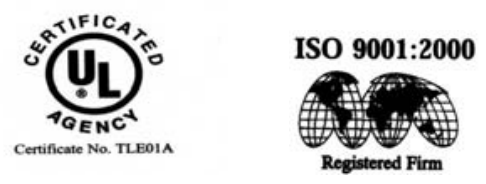


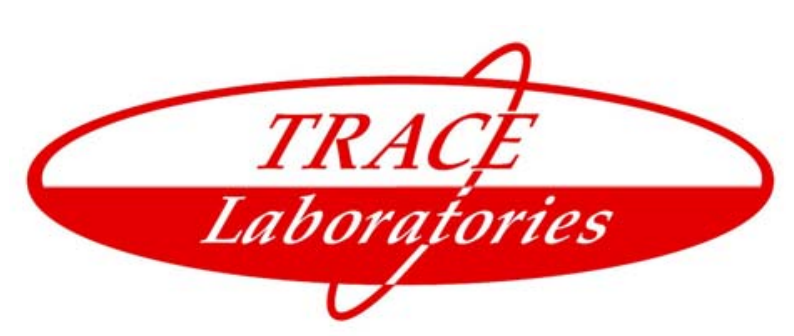

THERMAL SHOCK

TEST SPECIMENS:

Seven (7) groups of flexible printed circuit boards.

\section{REFERENCE:}

IPC-6013A, Amendment 2, Class 3, paragraph 3.10.2.

\section{REQUIREMENT:}

When specified on the procurement documentation, flexible printed wiring or test coupons shall be tested in accordance with the procedure outlined below.

The specimen shall be tested for thermal shock in accordance with IPC-TM-650, method 2.6.7.2B, except the temperature range shall be $-65^{\circ} \mathrm{C}$ to $125^{\circ} \mathrm{C}\left[-85^{\circ} \mathrm{F}\right.$ to $\left.257^{\circ} \mathrm{F}\right]$. Microsection evaluation in accordance with IPC-TM-650, Method 2.6.7.2 is not required. Following removal from the test chamber, the specimen shall meet the circuitry requirements of 3.9.2. The resistance value shall not vary by more than \pm $10 \%$.

\section{METHOD:}

Insulated wires were soldered to the terminals of the test specimen. The initial resistance measurement was obtained using a multimeter. The samples were placed in Thermal Shock Chamber. The cold chamber was set at $-65^{\circ} \mathrm{C}$, and the hot portion of the chamber was set at $125^{\circ} \mathrm{C}$. The dwell time was set at 15 minutes. The transfer to temperature extremes was approximately 15 seconds. During the first hot cycle, the resistance measurement was obtained with a multimeter.

The chamber was set for 100 cycles. During the last hot cycle the resistance measurement was again measured and recorded. The percent change was then calculated.

\section{RESULTS:}

Resistance Measurements:
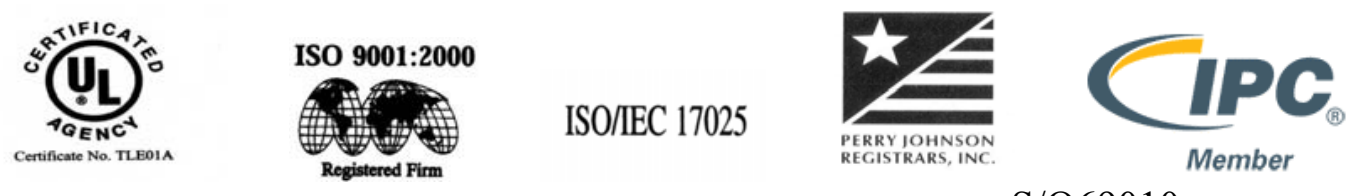


\section{TRACE
Laborafories}

\begin{tabular}{|c|c|c|c|c|}
\hline Group 1 & Layer & $\begin{array}{c}\text { Before } \\
\text { Conditioning } \\
\text { (milliohms) }\end{array}$ & $\begin{array}{c}\text { After } \\
\text { Conditioning } \\
\text { (milliohms) }\end{array}$ & \% Change \\
\hline-1 & 1 & 1.98 & 1.90 & 4.04 \\
\hline-1 & 2 & 8.65 & 8.54 & 1.27 \\
\hline-2 & 1 & 99.43 & 98.43 & 1.01 \\
\hline-2 & 2 & 85.00 & 84.36 & 0.75 \\
\hline-3 & 1 & 60.38 & 59.95 & 0.71 \\
\hline-3 & 2 & 112.00 & 111.00 & 0.89 \\
\hline
\end{tabular}

\begin{tabular}{|c|c|c|c|c|}
\hline Group 2 & Layer & $\begin{array}{c}\text { Before } \\
\text { Conditioning } \\
\text { (milliohms) }\end{array}$ & $\begin{array}{c}\text { After } \\
\text { Conditioning } \\
\text { (milliohms) }\end{array}$ & \% Change \\
\hline-1 & 1 & 25.52 & 25.11 & 1.61 \\
\hline-2 & 1 & 98.92 & 98.01 & 0.92 \\
\hline-2 & 2 & 83.77 & 83.07 & 0.84 \\
\hline-3 & 1 & 61.69 & 61.27 & 0.68 \\
\hline-3 & 2 & 104.17 & 102.78 & 1.33 \\
\hline
\end{tabular}

\begin{tabular}{|c|c|c|c|c|}
\hline Group 3 & Layer & $\begin{array}{c}\text { Before } \\
\text { Conditioning } \\
\text { (milliohms) }\end{array}$ & $\begin{array}{c}\text { After } \\
\text { Conditioning } \\
\text { (milliohms) }\end{array}$ & \% Change \\
\hline-1 & 1 & 2.17 & 2.08 & 4.15 \\
\hline-1 & 2 & 9.92 & 9.80 & 1.21 \\
\hline-2 & 1 & 106.92 & 105.88 & 0.97 \\
\hline-2 & 2 & 80.66 & 80.16 & 0.62 \\
\hline-3 & 1 & 58.42 & 57.84 & 0.99 \\
\hline-3 & 2 & 110.00 & 109.00 & 0.91 \\
\hline
\end{tabular}

\begin{tabular}{|c|c|c|c|c|}
\hline Group 4 & Layer & $\begin{array}{c}\text { Before } \\
\text { Conditioning } \\
\text { (milliohms) }\end{array}$ & $\begin{array}{c}\text { After } \\
\text { Conditioning } \\
\text { (milliohms) }\end{array}$ & \% Change \\
\hline-1 & 1 & 2.01 & 1.95 & 2.99 \\
\hline-1 & 2 & 9.69 & 9.56 & 1.34 \\
\hline-2 & 1 & 21.52 & 21.18 & 1.58 \\
\hline-3 & 1 & 60.54 & 59.98 & 0.93 \\
\hline-3 & 2 & 106.78 & 105.14 & 1.54 \\
\hline
\end{tabular}
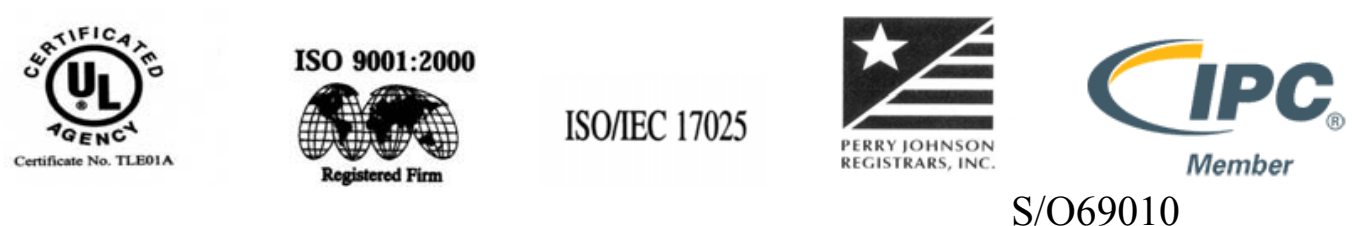


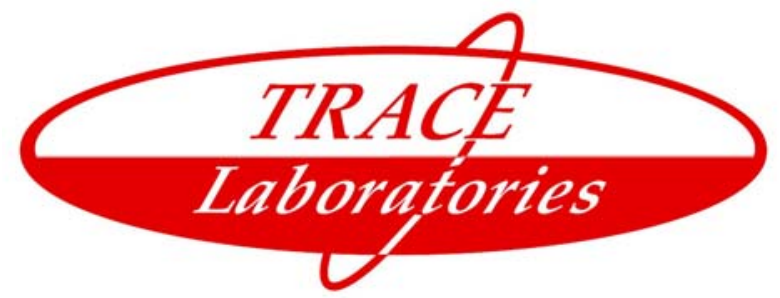

\begin{tabular}{|c|c|c|c|c|}
\hline Group 5 & Layer & $\begin{array}{c}\text { Before } \\
\text { Conditioning } \\
\text { (milliohms) }\end{array}$ & $\begin{array}{c}\text { After } \\
\text { Conditioning } \\
\text { (milliohms) }\end{array}$ & \% Change \\
\hline-1 & 1 & 2.04 & 1.96 & 3.92 \\
\hline-1 & 2 & 9.72 & 9.67 & 0.51 \\
\hline-2 & 1 & 26.58 & 26.21 & 1.39 \\
\hline-2 & 2 & 29.35 & 28.90 & 1.53 \\
\hline-3 & 1 & 23.78 & 23.43 & 1.47 \\
\hline-3 & 2 & 29.82 & 29.31 & 1.71 \\
\hline
\end{tabular}

\begin{tabular}{|c|c|c|c|c|}
\hline Group 6 & Layer & $\begin{array}{c}\text { Before } \\
\text { Conditioning } \\
\text { (milliohms) }\end{array}$ & $\begin{array}{c}\text { After } \\
\text { Conditioning } \\
\text { (milliohms) }\end{array}$ & \% Change \\
\hline-1 & 1 & 23.61 & 23.28 & 1.40 \\
\hline-2 & 1 & 106.05 & 104.80 & 1.18 \\
\hline-2 & 2 & 80.66 & 79.85 & 1.00 \\
\hline-3 & 1 & 23.97 & 23.61 & 1.50 \\
\hline-3 & 2 & 30.32 & 29.73 & 1.95 \\
\hline
\end{tabular}

\begin{tabular}{|c|c|c|c|c|}
\hline Group 7 & Layer & $\begin{array}{c}\text { Before } \\
\text { Conditioning } \\
\text { (milliohms) }\end{array}$ & $\begin{array}{c}\text { After } \\
\text { Conditioning } \\
\text { (milliohms) }\end{array}$ & \% Change \\
\hline-1 & 1 & 2.13 & 2.06 & 3.29 \\
\hline-1 & 2 & 9.79 & 9.68 & 1.12 \\
\hline-2 & 1 & 103.26 & 102.10 & 1.12 \\
\hline-2 & 2 & $*$ & $*$ & $*$ \\
\hline-3 & 1 & 60.80 & 60.17 & 1.04 \\
\hline-3 & 2 & 107.00 & 105.40 & 1.50 \\
\hline
\end{tabular}

* Board was shorted during the soldering process, prior to resistance readings.
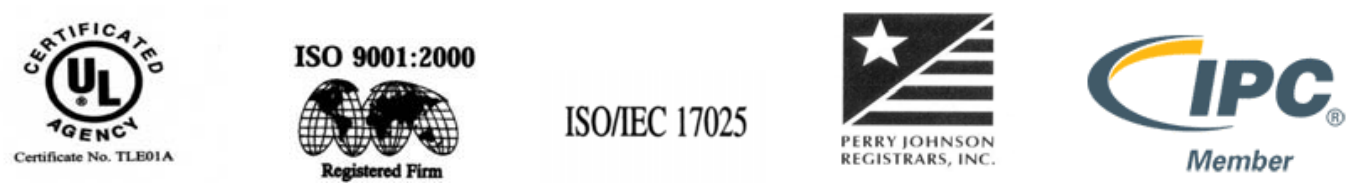


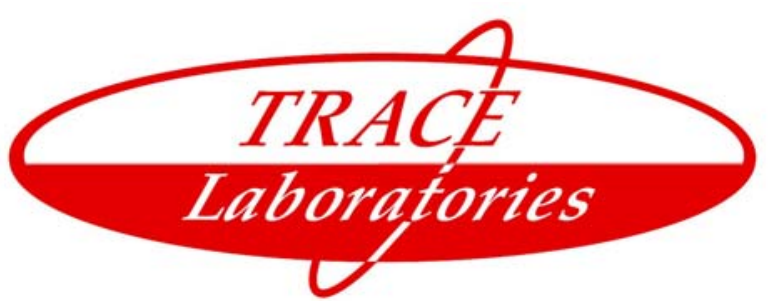

Trace Laboratories, Inc. certifies that the test equipment used complies with the calibration test purposes of ISO 10012-1, ANSI/NCSL Z540-1-1994, and MIL-STD-45662A and that the data contained in this report is accurate within the tolerance limitation of this equipment.

All test procedures detailed within this report are complete. The results in this report relate only to those items tested. If any additional information or clarification of this report is required, please contact us. This test report shall not be reproduced except in full, without the written approval of Trace Laboratories, Inc. Thank you for selecting Trace Laboratories, Inc. for your testing requirements.

Senior Engineer

Laboratory Technician

Laboratory Technician

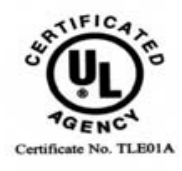

